\title{
Direct CP Violation in Charmless Hadronic B-Meson Decays at the PEP-II Asymmetric B-Meson Factory
}

\author{
By Alexandre Valerievich Telnov
}

Ph.D. Thesis, University of California, Berkeley 


\title{
Direct $C P$ Violation in Charmless Hadronic $B$-meson Decays at the PEP-II Asymmetric $B$-meson Factory
}

\author{
by \\ Alexandre Valerievich Telnov \\ B.Sc. (Novosibirsk State University, Novosibirsk, Russia) 1995 \\ A dissertation submitted in partial satisfaction of the \\ requirements for the degree of \\ Doctor of Philosophy \\ in \\ Physics \\ in the \\ GRADUATE DIVISION \\ of the \\ UNIVERSITY OF CALIFORNIA, BERKELEY
}

Committee in charge:

Professor Robert G. Jacobsen

Professor Marjorie D. Shapiro

Professor Jeffrey R. Long

Fall 2003 
The dissertation of Alexandre Valerievich Telnov is approved:

\begin{tabular}{lc}
\hline Chair & Date \\
& \\
\hline & Date \\
\\
\hline
\end{tabular}

Date

University of California, Berkeley

Fall 2003 


\title{
Direct $C P$ Violation in Charmless Hadronic $B$-meson Decays at the
} PEP-II Asymmetric $B$-meson Factory

\author{
Copyright 2003
}

by

Alexandre Valerievich Telnov 


\begin{abstract} PEP-II Asymmetric $B$-meson Factory

by

\author{
Alexandre Valerievich Telnov \\ Doctor of Philosophy in Physics \\ University of California, Berkeley \\ Professor Robert G. Jacobsen, Chair
}

Direct $C P$ Violation in Charmless Hadronic $B$-meson Decays at the

The study of the quark transition $b \rightarrow s \bar{s} s$, which is a pure loop-level ("penguin") process leading to several $B$-meson-decay final states, most notably $\phi K$, is arguably the hottest topic in $B$-meson physics today. The reason is the sensitivity of the amplitudes and the $C P$-violating asymmetries in such processes to physics beyond the Standard Model. By performing these measurements, we improve our understanding of the phenomenon of combined-parity $(C P)$ violation, which is believed to be responsible for the dominance of matter over antimatter in our Universe.

Here, we present measurements of branching fractions and charge asymmetries in the decays $B^{+} \rightarrow \phi K^{+}$and $B^{0} \rightarrow \phi K^{0}$ in a sample of approximately 89 million $B \bar{B}$ pairs collected by the BABAR detector at the PEP-II asymmetric-energy $B$-meson Factory at SLAC. We determine $\mathcal{B}\left(B^{+} \rightarrow \phi K^{+}\right)=\left(10.0_{-0.8}^{+0.9} \pm 0.5\right) \times 10^{-6}$ and $\mathcal{B}\left(B^{0} \rightarrow\right.$ $\left.\phi K^{0}\right)=\left(8.4_{-1.3}^{+1.5} \pm 0.5\right) \times 10^{-6}$, where the first error is statistical and the second is 
systematic. Additionally, we measure the $C P$-violating charge asymmetry $\mathcal{A}_{C P}\left(B^{ \pm} \rightarrow\right.$ $\left.\phi K^{ \pm}\right)=0.04 \pm 0.09 \pm 0.01$, with a $90 \%$ confidence-level interval of $[-0.10,0.18]$, and set an upper limit on the CKM- and color-suppressed decay $B^{+} \rightarrow \phi \pi^{+}, \mathcal{B}\left(B^{+} \rightarrow\right.$ $\left.\phi \pi^{+}\right)<0.41 \times 10^{-6}$ (at the $90 \%$ confidence level).

Our results are consistent with the Standard Model, which predicts $\mathcal{A}_{C P}\left(B^{ \pm} \rightarrow\right.$ $\left.\phi K^{ \pm}\right) \lesssim 1 \%$ and $\mathcal{B}(B \rightarrow \phi \pi) \ll 10^{-7}$. Since many models of physics beyond the Standard Model introduce additional loop diagrams with new heavy particles and new $C P$-violating phases that would contribute to these decays, potentially making $\mathcal{A}_{C P}$ $\left(B^{ \pm} \rightarrow \phi K^{ \pm}\right)$and $\mathcal{B}(B \rightarrow \phi \pi)$ quite large, our results can be used to substantially constrain the parameter spaces of such models.

Professor Robert G. Jacobsen

Dissertation Committee Chair 
I dedicate my dissertation to my mother, Lilia Yakovlevna, my father, Valery Ivanovich, and, most importantly, to my maternal grandmother, Maria Stepanovna.

Additionally, I dedicate my dissertation to the creators and builders of Akademgorodok, a town of Science in the Siberian taiga on the shores of the Ob Sea, and to all the people who came to Akademgorodok and dedicated their lives to its success. 


\section{Contents}

1 An Introduction for the Layman 1

1.1 A Foreword $\ldots \ldots \ldots \ldots \ldots \ldots \ldots \ldots$

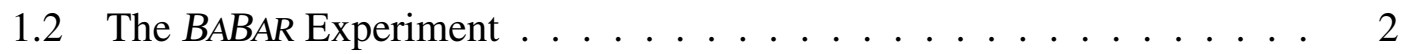

1.3 Particles and Antiparticles $\ldots \ldots \ldots \ldots \ldots$

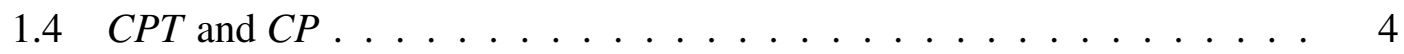

1.5 Creation and Annihilation of Particle-Antiparticle Pairs . . . . . . . 6

1.6 The Big-Bang Connection $\ldots \ldots \ldots \ldots \ldots$

1.7 Sakharov's Three Conditions $\ldots \ldots \ldots \ldots \ldots$

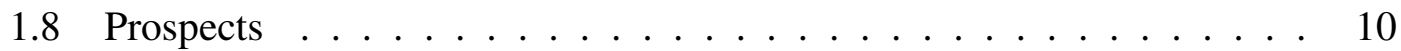

2 Theoretical Introduction $\quad 11$

$2.1 C P$ violation in the Standard Model $\ldots \ldots \ldots \ldots$

2.2 Pure-penguin $B$-meson decays: a window to physics beyond the Standard Model . . . . . . . . . . . . . . . . . . . . 15

3 Instrumentation and the Data Set $\quad 20$ 
3.1 The PEP-II $B$-meson factory . . . . . . . . . . . . 20

3.2 Overview of the BABAR detector . . . . . . . . . . . 26

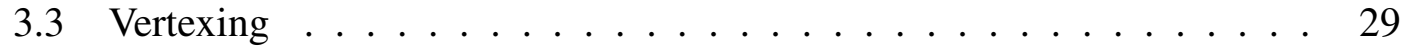

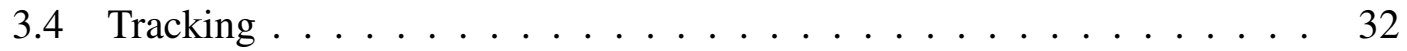

3.5 Charged-particle identification ............... 36

3.6 The Electromagnetic Calorimeter . . . . . . . . . . . . . . 40

3.7 The Instrumented Flux Return _. . . . . . . . . . . . . . . . 41

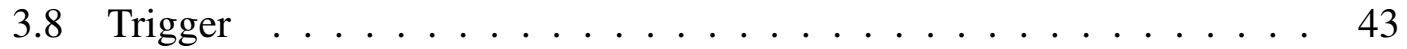

3.9 The data set . . . . . . . . . . . . . . . . . 44

4 Terminology 446

4.1 Variables ............................ 46

4.2 Lists ........................... 57

4.3 Other terminology . . . . . . . . . . . . . . . . 58

5 Maximum-Likelihood Fit 59

6 Event Selection and Preliminary Cuts 63

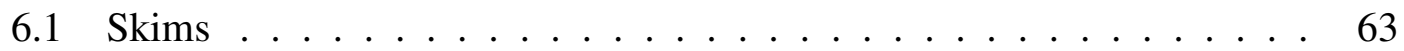

6.2 Reconstruction of $B$ meson candidates . . . . . . . . . . . . 64

6.3 Selection of events . . . . . . . . . . . . . 65

$\begin{array}{lll}7 & \text { Calibration Modes } & 70\end{array}$

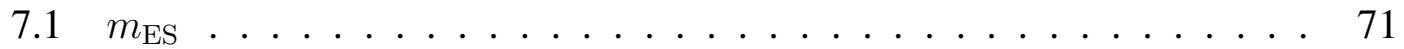


$7.2 \Delta E$ and the tracking bias in runs $18000-22929 \ldots \ldots$. . . . . . 74

7.3 Fisher Discriminant $(\mathcal{F}) \quad \ldots \ldots \ldots \ldots$

8 Probability Distribution Functions $\quad 82$

$8.1 m_{\mathrm{ES}} \ldots \ldots \ldots \ldots \ldots$

$8.2 \Delta E \ldots \ldots \ldots \ldots \ldots \ldots$

8.3 Fisher discriminant . . . . . . . . . . . . . . . . 90

8.4 Shape of the $\phi$ resonance . . . . . . . . . . . . . . . . . 90

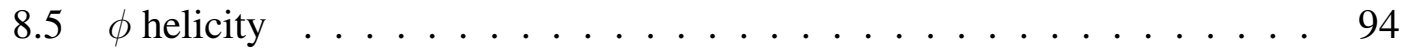

9 Efficiency and Production Rate 99

9.1 Reconstruction and ML fit efficiencies . . . . . . . . . . . . . . . 99

9.2 Tracking efficiency . . . . . . . . . . . . . . . 102

$9.3 K_{S}^{0}$ reconstruction efficiency in $B^{0} \rightarrow \phi K_{S}^{0} \ldots \ldots \ldots 3$

9.4 Particle identification efficiency . . . . . . . . . . . . . . . 104

$9.5 \Upsilon(4 S)$ and daughter branching fractions . . . . . . . . . . 107

10 Physics Results $\quad 108$

11 Cross Checks 111

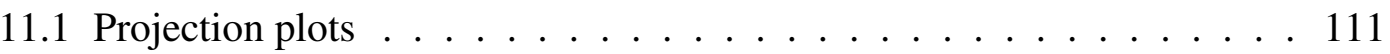

$11.2 \pi^{ \pm} / K^{ \pm}$separation in data . . . . . . . . . . . . . 115

11.2.1 $\Delta E$ separation . . . . . . . . . . . . . 115

11.2.2 DIRC Cherenkov angle pull $\pi^{ \pm} / K^{ \pm}$separation . . . . . . . . 120 
11.3 Off-resonance data sample and generic continuum MC . . . . . . . 122

11.4 Generic $B \bar{B}$ Monte Carlo and the Charmless $B \bar{B}$ cocktail . . . . . . . 122

11.4.1 Generic $B \bar{B}$ background to $B^{ \pm} \rightarrow \phi K^{ \pm} \ldots \ldots 123$

11.4.2 Generic $B \bar{B}$ background to $B^{ \pm} \rightarrow \phi \pi^{ \pm} \ldots \ldots$. . . . . . . . 124

11.4.3 Generic $B \bar{B}$ background to $B^{0} \rightarrow \phi K_{S}^{0} \ldots \ldots . \ldots 125$

11.4.4 Conclusion of the generic $B \bar{B}$ MC study $\ldots \ldots$. . . . . . 126

11.5 Exclusive $B$ decay modes: $B \rightarrow \phi K^{*}, 3$-body, etc. . . . . . . . . 126

11.5.1 Cross feed from $B \rightarrow \phi K^{*}$ modes . . . . . . . . . . . 126

11.5.2 Cross feed from non-resonant three-body decays . . . . . . 128

11.5.3 Cross feed of $B \rightarrow \phi h$ modes into each other . . . . . . . . 129

$11.6 B \bar{B}$ background: conclusion . . . . . . . . . . . . . . . . 129

11.7 "Goodness-of-fit" tests . . . . . . . . . . . . . . . 130

12 Systematic Studies $\quad 131$

12.1 Uncertainty of PDF parameters . . . . . . . . . . . . 131

12.2 The systematic error on $\mathcal{A}_{C P}\left(B^{ \pm} \rightarrow \phi K^{ \pm}\right) \ldots \ldots$. . . . . . . 133

12.3 Other sources of systematic errors . . . . . . . . . . . 136

13 Other Direct- $C P$ Results from BABAR 137

14 Summary $\quad 140$

$\begin{array}{lll}\text { A Miscellanea } & 141\end{array}$

A.1 Terminology of Experimental Particle Physics . . . . . . . . . . . . . 141 
A.2 BABAR-related terminology . . . . . . . . . . . . 143

A.3 Functions . . . . . . . . . . . . . . . . . . 144

A.4 Theoretical description of resonance shapes . . . . . . . . . . . 145

$\begin{array}{llr}\text { B Input-variable correlation matrices } & 147\end{array}$

B.1 Correlation matrices in $B^{ \pm} \rightarrow \phi h^{ \pm} \ldots \ldots \ldots$. . . . . . . . . 147

B.2 Correlation matrices in $B^{0} \rightarrow \phi K_{S}^{0} \ldots \ldots \ldots$. . . . . . . 148

C $\quad K_{S}^{0} \rightarrow \pi^{+} \pi^{-}$Reconstruction Efficiency: Release-10 Data vs. SP4 MC 151

C.1 "Executive summary" . . . . . . . . . . . . . . . . 151

C.2 Introduction . . . . . . . . . . . . . . . 152

C.3 Method ....................... 154

C.3.1 Data set and selection of $K_{S}^{0} \rightarrow \pi^{+} \pi^{-}$candidates . . . . . . . 154

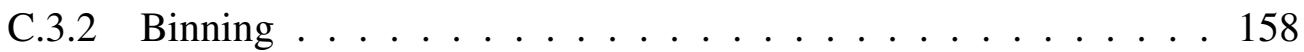

C.3.3 Calculation of the number of $K_{S}^{0}$ s s in each bin . . . . . . 160

C.3.4 Calculation of the efficiency table elements . . . . . . . . . 164

C.3.5 Treatment of special cases . . . . . . . . . . . 166

C.4 Determination of the mode-dependent data/MC overall efficiency ratio . 167

C.5 Test modes and systematics studies . . . . . . . . . . . 169

C.6 Results . . . . . . . . . . . . . . . . . . 170

C.7 Summary . . . . . . . . . . . . . . . . . 174

C.8 The Recipe . . . . . . . . . . . . . . . . . . . . 174

C.8.1 Files and Tools . . . . . . . . . . . . . . . . . 174 
C.8.2 Give me the recipe already! . . . . . . . . . . . . 181

C.9 Notes on the $\chi^{2}$ and ML histogram-fitting methods . . . . . . . 182 


\section{List of Figures}

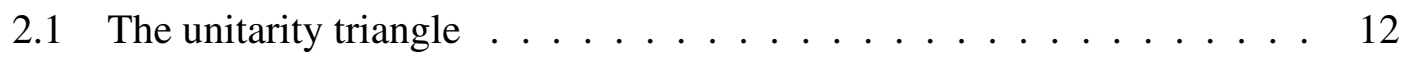

2.2 Current constraints on the unitarity triangle . . . . . . . . . 14

2.3 Example of a tree-level diagram: $B \rightarrow J / \psi K \ldots \ldots \ldots \ldots$

2.4 Examples of gluonic penguin diagrams for $B \rightarrow \phi K$ and $B \rightarrow \phi \pi \ldots 16$

2.5 Electroweak penguin diagrams for $B^{ \pm} \rightarrow \phi K^{ \pm} \ldots \ldots \ldots \ldots$

2.6 The rescattering diagram for $B \rightarrow \phi K$ and $B \rightarrow \phi \pi \ldots \ldots \ldots$

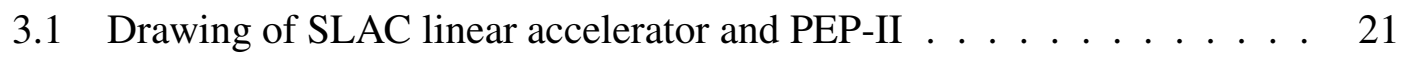

3.2 Drawing of the PEP-II interaction region . . . . . . . . . 22

3.3 PEP-II scan of the $\Upsilon(4 S)$ resonance $\ldots \ldots \ldots \ldots \ldots$

3.4 PEP-II-delivered and BABAR-recorded integrated luminosities $\ldots \ldots 25$

3.5 Side view of the $B A B A R$ detector $\ldots \ldots \ldots \ldots \ldots$

3.6 Side view of the BABAR detector $\ldots \ldots \ldots \ldots \ldots$

3.7 Schematic view of the SVT $\ldots \ldots \ldots \ldots \ldots \ldots \ldots \ldots$

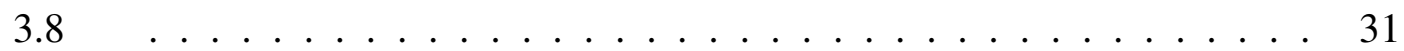

3.9 Schematic view of the $\mathrm{DCH} \ldots \ldots \ldots \ldots \ldots \ldots$ 
3.10 Schematic layout of $\mathrm{DCH}$ cells $\ldots \ldots \ldots \ldots$

3.11 Illustration of DCH drift-cell isochrones . . . . . . . . . . . . . 35

$3.12 \mathrm{~d} E / \mathrm{d} x$ measurement in $\mathrm{DCH} \ldots \ldots \ldots \ldots \ldots \ldots \ldots$

3.13 Illustration of the DIRC principle . . . . . . . . . . . 37

3.14 Schematic 3-D view of the DIRC $\ldots \ldots \ldots \ldots$

3.15 Schematic drawing of the EMC . . . . . . . . . . . . . . 41

3.16 Cross section of an IFR RPC $\ldots \ldots \ldots \ldots \ldots$

$4.1 m_{\mathrm{ES}}$ and $m_{\mathrm{EC}}$ distributions in the on-resonance sideband $\ldots \ldots . .48$

4.2 $\Delta E$ versus (left) $m_{\mathrm{ES}}$ and (right) $m_{\mathrm{EC}}$ for $B^{ \pm} \rightarrow \phi h^{ \pm}$candidates in off-resonance data . . . . . . . . . . . . . . . . . . . . . . . . . . 49

4.3 $\left|\cos \theta_{T}\right|$ distributions for $B^{ \pm} \rightarrow \phi K^{ \pm}$in signal and in continuum background $\ldots \ldots \ldots \ldots \ldots \ldots \ldots \ldots \ldots$

$4.4\left|\cos \theta_{T}\right|$ distributions for $B^{ \pm} \rightarrow \phi h^{ \pm}$candidates in $u \bar{u}+d \bar{d}+s \bar{s}, c \bar{c}$,

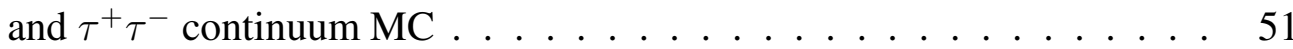

4.5 $\left|\cos \theta_{B_{\mathrm{thr}}}\right|$ distributions for signal and for continuum background $\ldots . \quad 53$

4.6 $\left|\cos \theta_{B_{\mathrm{thr}}}\right|$ distributions $u \bar{u}+d \bar{d}+s \bar{s}, c \bar{c}$, and $\tau^{+} \tau^{-}$continuum MC . $\quad 54$

4.7 Comparison of the CLEO Fisher and the "BABAR" Fisher discriminants . 56

6.1 Event display of a likely $B^{0} \rightarrow \phi K_{S}^{0}$ candidate event $\ldots \ldots \ldots 66$

7.1 $m_{\mathrm{ES}}$ distributions for $B^{+} \rightarrow \pi^{+} \bar{D}^{0}\left(\bar{D}^{0} \rightarrow K^{+} \pi^{-}\right)$and $B^{0} \rightarrow \pi^{+} D^{-}\left(D^{-} \rightarrow\right.$ $\left.K^{0} \pi^{-}\right)$signal MC . . . . . . . . . . . . . . . 71 
7.2 $m_{\mathrm{ES}}$ distributions in the on-resonance signal region for $B^{+} \rightarrow \pi^{+} \bar{D}^{0}\left(\bar{D}^{0} \rightarrow\right.$ $\left.K^{+} \pi^{-}\right), B^{0} \rightarrow \pi^{+} D^{-}\left(D^{-} \rightarrow K^{0} \pi^{-}\right), B^{+} \rightarrow \pi^{+} \bar{D}^{0}\left(\bar{D}^{0} \rightarrow K^{+} K^{-}\right)$, and $B^{+} \rightarrow \pi^{+} \bar{D}^{0}\left(\bar{D}^{0} \rightarrow \pi^{+} \pi^{-}\right) \ldots \ldots \ldots \ldots \ldots \ldots$

7.3 Uncorrected $\Delta E$ distributions for positively and negatively charged $B^{+} \rightarrow$ $\pi^{+} \bar{D}^{0}\left(\bar{D}^{0} \rightarrow K^{+} \pi^{-}\right)$candidates in data . . . . . . . . . 75

7.4 $\Delta E$ distributions for $B^{+} \rightarrow \pi^{+} \bar{D}^{0}\left(\bar{D}^{0} \rightarrow K^{+} \pi^{-}\right)$and $B^{0} \rightarrow \pi^{+} D^{-}\left(D^{-} \rightarrow\right.$ $\left.K^{0} \pi^{-}\right)$in signal MC $\ldots \ldots \ldots \ldots \ldots \ldots$

7.5 $\Delta E$ distributions for $B^{+} \rightarrow \pi^{+} \bar{D}^{0}\left(\bar{D}^{0} \rightarrow K^{+} \pi^{-}\right)$and $B^{0} \rightarrow \pi^{+} D^{-}\left(D^{-} \rightarrow\right.$ $\left.K^{0} \pi^{-}\right)$in the on-resonance signal region . . . . . . . . . . 78

7.6 Fisher discriminant distributions for $B^{+} \rightarrow \pi^{+} \bar{D}^{0}\left(\bar{D}^{0} \rightarrow K^{+} \pi^{-}\right)$in SP4 MC and in data . . . . . . . . . . . . . . 80

8.1 Definition of the on-resonance "grand" sideband in $m_{\mathrm{ES}}$ and $\Delta E \ldots \quad$. . 83

8.2 $m_{\mathrm{ES}}$ distributions for $B^{ \pm} \rightarrow \phi K^{ \pm}$signal $\mathrm{MC}$ and for the $B^{ \pm} \rightarrow \phi h^{ \pm}$

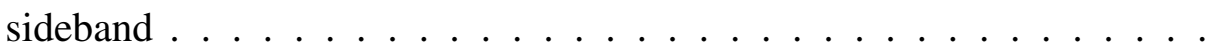

8.3 $m_{\mathrm{ES}}$ distributions for $B^{0} \rightarrow \phi K_{S}^{0}$ signal $\mathrm{MC}$ and for the $B^{0} \rightarrow \phi K_{S}^{0}$ sideband . . . . . . . . . . . . . . . . . . . . . . 85

8.4 $\Delta E$ distributions for $B^{ \pm} \rightarrow \phi K^{ \pm} \mathrm{SP} 4$ signal $\mathrm{MC}$ and for the $B^{ \pm} \rightarrow$ $\phi h^{ \pm}$on-resonance sideband $\ldots \ldots \ldots \ldots \ldots$

8.5 $\Delta E$ distributions for $B^{0} \rightarrow \phi K_{S}^{0} \mathrm{SP} 4$ signal MC and for the $B^{0} \rightarrow \phi K_{S}^{0}$ on-resonance sideband $\ldots \ldots \ldots \ldots \ldots \ldots$ 
8.6 $\mathcal{F}$ distributions for $B^{ \pm} \rightarrow \phi K^{ \pm}$signal MC and for the $B^{ \pm} \rightarrow \phi h^{ \pm}$ on-resonance sideband . . . . . . . . . . . . . . . . 99

8.7 $\mathcal{F}$ distributions for $B^{0} \rightarrow \phi K_{S}^{0}$ signal MC and for the $B^{0} \rightarrow \phi K_{S}^{0}$ onresonance sideband . . . . . . . . . . . . . . .

8.8 $m_{K K}$ distributions in $B^{ \pm} \rightarrow \phi K^{ \pm} \mathrm{SP} 4$ signal Monte Carlo, the $B^{ \pm} \rightarrow$ $\phi h^{ \pm}$sideband, and the $B^{0} \rightarrow \phi K_{S}^{0}$ sideband . . . . . . . . . . 95

8.9 $\mathcal{H}$ distributions for $B^{ \pm} \rightarrow \phi K^{ \pm}$SP4 signal MC and the $B^{ \pm} \rightarrow \phi h^{ \pm}$ on-resonance sideband . . . . . . . . . . . . . . . 97

$8.10 \mathcal{H}$ distributions for $B^{0} \rightarrow \phi K_{S}^{0}$ SP4 signal MC and the $B^{0} \rightarrow \phi K_{S}^{0}$ on-resonance sideband . . . . . . . . . . . . . . . 98

9.1 Laboratory-frame momentum spectra of $\phi \rightarrow K^{+} K^{-}$kaons in $B \rightarrow$ $\phi K$ and of all ChargedTracks, GoodTracksVeryLoose, GoodTracksLoose, and GoodTracksTight . . . . . . . . . . . . . 106

11.1 Projection plots for the variable $m_{\mathrm{ES}}$ in $B^{ \pm} \rightarrow \phi K^{ \pm} \ldots \ldots$. . . . . 113

11.2 Projection plots for the variables $\Delta E, m_{K K}, \cos \theta_{\text {hel }}$ in $B^{ \pm} \rightarrow \phi K^{ \pm}$. 114

11.3 Projection plots for the Fisher discriminant $\mathcal{F}$ and the Cherenkov angle pull in $B^{ \pm} \rightarrow \phi K^{ \pm} \ldots \ldots \ldots \ldots$. . . . . . . . . . . . . . . . . . . .

11.4 Projection plots for the variables $m_{\mathrm{ES}}$ and $\Delta E$ in $B^{0} \rightarrow \phi K_{S}^{0} \ldots \ldots 116$

11.5 Projection plots for $m_{K^{+} K^{-}}$extended to $1.2 \mathrm{GeV} / c^{2} \ldots \ldots$. . . . . 116 
11.6 Projection plots of the variables $m_{\mathrm{ES}}(\mathrm{a}, \mathrm{c})$ and $\Delta E(\mathrm{~b}, \mathrm{~d})$ in the fit for the $\phi K^{+}$(top) and $\phi K_{S}^{0}\left(\pi^{+} \pi^{-}\right)$(bottom) branching fractions. The data are shown by the histogram, while the curve is the result of the fit. The signal-to-background ratio in the figure is enhanced with a requirement on the event probability that is less stringent than that in Eq. 11.1. . . 117

11.7 Projection plots for the variables $m_{\mathrm{ES}}, \Delta E$ and the Cherenkov angle pull in $B^{ \pm} \rightarrow \phi \pi^{ \pm} \ldots \ldots \ldots \ldots \ldots \ldots \ldots$

11.8 Projection plots for the variables $m_{K K}, \cos \theta_{\text {hel }}$ and the Fisher discriminant $\mathcal{F}$ in $B^{0} \rightarrow \phi K_{S}^{0} \ldots \ldots \ldots \ldots \ldots \ldots$

11.9 Plots illustrating $\pi^{ \pm} / K^{ \pm}$separation in $B A B A R \ldots \ldots \ldots \ldots$

C.1 Distributions of the $\pi^{+} \pi^{-}$invariant mass and the polar angle $\theta_{\text {lab }}$ in data and $\mathrm{MC} \ldots \ldots \ldots \ldots \ldots \ldots \ldots \ldots$

C.2 Distributions of the $K_{S}^{0}$ transverse momentum and the $K_{S}^{0}$ momentum in the $\Upsilon(4 S)$ center-of-mass frame in data and MC $\ldots \ldots \ldots 156$

C.3 Distribution of the $K_{S}^{0}$ transverse flight length $d_{X Y} \ldots \ldots \ldots \ldots 157$

C.4 Examples of fitted $K_{S}^{0} \rightarrow \pi^{+} \pi^{-}$invariant-mass histograms $\ldots \ldots \ldots 161$

C.5 $\quad K_{S}^{0}$ reconstruction efficiency correction for $B^{0} \rightarrow \phi K_{S}^{0}$ as a function of the transverse flight length $d_{X Y} \ldots \ldots \ldots \ldots \ldots \ldots 17$

C.6 $K_{S}^{0}$ reconstruction efficiency correction for $B^{0} \rightarrow \pi^{+} D^{-}\left(D^{-} \rightarrow K_{S}^{0} \pi^{-}\right)$ as a function of the transverse flight length $d_{X Y} \ldots \ldots \ldots 173$ 


\section{List of Tables}

7.1 Fit results for calibration-mode $m_{\mathrm{ES}}$ distributions in SP4 Monte Carlo and in data ............................. 73

7.2 Fit results for calibration-mode $\Delta E$ distributions in SP4 MC and in data 79

8.1 Fit results for $m_{\mathrm{ES}}$ PDFs in SP4 signal $\mathrm{MC}$ and in the on-resonance

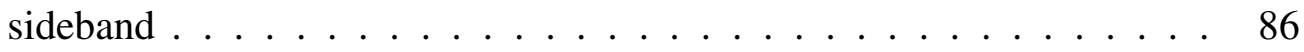

8.2 Fit results for $\Delta E$ PDFs in SP4 signal $\mathrm{MC}$ and in the on-resonance sideband . . . . . . . . . . . . . . . . . . . . . 89

8.3 Fit results for Fisher discriminant PDFs in SP4 signal MC and in the on-resonance sideband . . . . . . . . . . . . . . 92

8.4 Parameters of the shape of the fake $\phi$ background and the fraction of real $\phi$ 's in the on-resonance sideband . . . . . . . . . . . . . . . . 94

8.5 Fit results for $\mathcal{H}$ distributions for real and fake $\phi$ 's in the on-resonance

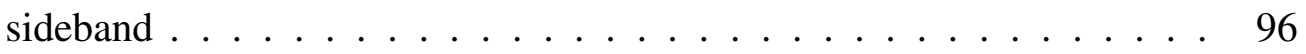

9.1 Determination of reconstruction and ML-fit efficiencies . . . . . . . . 100 
10.1 Maximum-likelihood fit results for the modes $B^{ \pm} \rightarrow \phi K^{ \pm}$and $B^{ \pm} \rightarrow$

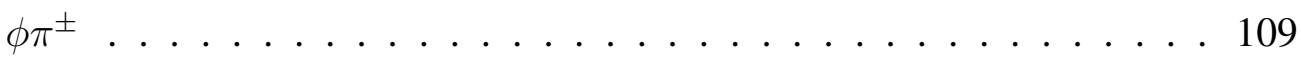

10.2 Maximum-likelihood fit results for the mode $B^{0} \rightarrow \phi K_{S}^{0} \ldots \ldots$. . . . 110

11.1 Cross feed from suspect $B$ decay modes into the modes $B^{ \pm} \rightarrow \phi K^{ \pm}$, $B^{ \pm} \rightarrow \phi \pi^{ \pm}$, and $B^{0} \rightarrow \phi K_{S}^{0} \ldots \ldots \ldots \ldots$

12.1 Summary of sources of systematic uncertainties to $B \rightarrow \phi h$ branching

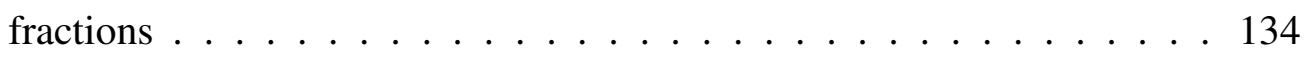

13.1 Summary of direct- $C P$-violation results, part I . . . . . . . . . . . 138

13.2 Summary of direct- $C P$-violation results, part II . . . . . . . . . . . 139

B.1 Correlation matrix for $B^{ \pm} \rightarrow \phi K^{ \pm}$SP4 signal Monte Carlo . . . . . . . 148

B.2 Correlation matrix for $B^{ \pm} \rightarrow \phi K^{ \pm}$on-resonance data . . . . . . . . . . 149

B.3 Correlation matrix for $B^{0} \rightarrow \phi K_{S}^{0} \mathrm{SP} 4$ signal Monte Carlo . . . . . . . 149

B.4 Correlation matrix for $B^{0} \rightarrow \phi K_{S}^{0}$ on-resonance data $\ldots . . . . . .150$

C.1 Summary of the Release-10 data and SP4 Monte Carlo data sets used in the $K_{S}^{0}$ reconstruction efficiency study $\ldots \ldots \ldots \ldots$

C.2 Central values of the $K_{S}^{0}$ mass distributions in Release-10 data and SP4

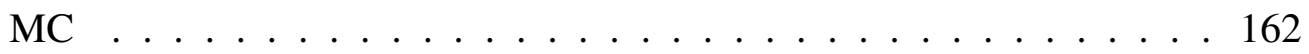

C.3 Correction factors for "coarse" binning . . . . . . . . . . . . . 175

C.4 Correction factors for "alternative" binning . . . . . . . . . 176

C.5 Correction factors for "fine" binning . . . . . . . . . . . . . . . 177 


\section{Acknowledgements}

Growing up as a physicist alongside the BABAR experiment, from its infancy to maturity, has been an absolutely wonderful, exciting and fruitful experience. I am very grateful to David Hitlin for having been the leading force in making BABAR a reality, and to all the other BABAR collaborators who, through years of long days (and nights), contributed to making BABAR such a smashing success.

In the Fall 1996 edition of the UC Berkeley Physics newsletter, Bob Jacobsen, my faculty research advisor, argued why the best place to do physics at SLAC is Berkeley. I could not agree more! I am grateful to Moishe Pripstein, who has been the principal architect of the collegial and productive environment of the BABAR group at LBNL, always able and willing to dispense thoughtful—and sometimes invaluable — advice to his younger colleagues. I am also thankful to Bob Cahn, Marjorie Shapiro, David Brown, Vasilii Shelkov, Janice Button-Shafer, and many other people at the Berkeley Lab whose input helped me in my studies. Yuriy Groysman and Gennadiy Kukartsev have been wonderful officemates and great friends.

Key to becoming an established member of the $B A B A R$ collaboration were my contributions to the DIRC gas/water system effort, led by Richard Kadel, to the deployment and management of the BABAR online and console farms, led by Tom Glanzman, and to the PEP-II background remediation efforts in the early days of data taking, led by Guy Wormser and Terry Geld. I am grateful for the trust and guidance extended to me by Mike Sokoloff, Bill Wisniewski, and Eli Rosenberg during my six-month term as the 
$B A B A R$ deputy run coordinator.

A far as the physics analysis contained in this dissertation is concerned, my foremost gratitude goes to Andrei Gritsan, who has been the leading organizer and contributor to the analysis effort at LBNL for the past three years. My thanks also go to Paul Harrison for inspiring me to look at charmless $B$-meson decays for a possible topic of my dissertation research, to Sergei Ganzhur, Theresa Harrison, and Andy Salnikov, whose help was very important in getting me started with the BABAR physics-analysis code, and to Bill Ford and Jim Smith for their tireless efforts in leading the quasi-twobody analysis working group and keeping it organized. I also appreciate having being frequently asked by David Leith, and, especially, Richard Kadel and Vera Lüth, "Is your dissertation done yet?"

I also feel compelled to acknowledge here the great teachers that I had at my high school and at Novosibirsk State University. I would like to mention by name Evgenii Bichenkov and Evgenii Palchikov, who have for the past two decades been carrying with excellence the burden of teaching first freshman physics courses at the NSU; Valery Serbo and Iosif Khriplovich, Vladimir Ivanov and David Shapiro for being the kind of lecturers in, respectively, physics and mathematics that an undergraduate student anywhere in the world can possibly dream of; Yury Eidelman, the best discussion-section instructor ever; and Alexei Maslennikov, who directed my diploma project. Special thanks to Igor Kotelnikov, who was not only a great lecturer and then Dean of the Physics Department, but also the author of my favorite book on $\mathrm{LT}_{\mathrm{E}} \mathrm{X}$.

My graduate-student experience at Berkeley would have been incomplete without 
the three years at the International House, Berkeley, and the dozens of people from all over the world who I befriended there: Merijn Rengers, Pyotr Yeltsov, Sergio Iavicoli, Kumi Furuyashiki, Ilya Yakubovich, Luiza Miranian, Andris Ambainis, Liza Mark, and many, many others. Thank you, Joe Lurie, for making I-House an ever more wonderful place for international students to get integrated into life in America!

For the past five years, my favorite pastime has been playing Chto? Gde? Kogda? (What? Where? When?), an intellectual game somewhat similar to College Bowl that has been extraordinarily popular in Russia and Russian-speaking communities around the world for the past thirty years. Dima Teytelman, Masha Sokolova, Ilya Mironov, Lyonya Kolmanovsky, Vitaly Kolmanovsky, and, of course, the captain of the TOPMO3A team, dear Leonid Mikhailovich Litvak: spending time with you cracking those tricky questions and puzzles is so much fun! 


\section{Chapter 1}

\section{An Introduction for the Layman}

\subsection{A Foreword}

In this first Chapter of my doctoral dissertation, the only one targeted at the audience with no specific training in particle physics, I shall endeavor to acquaint the reader with the nature of my scientific pursuits and the current state of my field of study. It is not my intent to tell the whole story, for this would be impossible within the space and time confines available to me-my intent is to get the reader excited about the things that I and my colleagues do, and, hopefully, make the reader want to learn more.

The discussion in this Chapter shall be based only on the facts about science that an average educated American can reasonably be expected to have had exposure to via the sources such as PBS, The New York Times, The Economist, and Star Trek. No math and no jargon are used here, but I do mention physics phenomena that the reader may have never heard about before; should this be the case, my advice is to keep on reading this 
Chapter to the end and read up on these unfamiliar phenomena soon afterwards.

\subsection{The $B_{A} B_{A R}$ Experiment}

Before we go into discussing physics, let me say a few words about $B A B A R$, the major international particle-physics experiment at which the results presented in this dissertation were obtained.

In early 1990s, in responce to the strong desire of the particle-physics community to have at its disposal an electron-positron collider able to produce tens of millions of $\Upsilon(4 S)$ particles a year, governments of nine countries (Canada, China, France, Germany, Italy, Norway, Russia, the United Kingdom, and the United States) joined forces in funding the construction of an approximately \$350-million accelerator/detector complex at the Stanford Linear Accelerator Center (SLAC), a Unites States Department of Energy national laboratory located near Palo Alto, California, and operated by Stanford University. Close to 600 physicists from over 70 universities and national laboratories, slightly over half of them non-U.S., collaborated to build and now operate the particle detector; the accelerator is primarily the responsibility of SLAC.

Build in the tunnel and reusing some of the parts of the defunct PEP electronpositron collider, the accelerator complex was appropriately named PEP-II, or, more officially, the "PEP-II $B$ Factory". Here $B$ refers to the $B$ mesons, particles that contain one light quark (up or down) and one beauty (or bottom) antiquark. Created in a collision of an electron and a positron, $e^{+} e^{-} \rightarrow \Upsilon(4 S)$, the $\Upsilon(4 S)$ resonance promptly 
decays into a meson-antimeson pair, $\Upsilon(4 S) \rightarrow B \bar{B}$; it is the $B$ mesons that physicists are after. In turn, the word "factory" reflects the fact that the rate of production of $B \bar{B}$ pairs at PEP-II is greater than one per second.

Then came the question, "How to we name the detector of our new $B$ factory?!" The most logical name, "B Factory Detector," would obviously not fly with English speakers because of the highly suggestive acronym. “ $B \bar{B} \ldots$ Bi-Bi-bar. . BABAR!!!”... We do not know for sure who first came up with the idea of naming the detector of the new $B$ meson factory after Babar, the famous character of children's books written decades ago by the French author Jean de Brunhoff and his son Laurent de Brunhoff. In any case, a permission to use the names and the distinctive likenesses of Babar and members of his royal family (Celeste, Cornelius, Flora, Pom, Alexander, and others), was quite easily obtained from Laurent de Brunhoff; current copyright holder is Nelvana, a major animation production and distribution company.

\subsection{Particles and Antiparticles}

Equations of Quantum Field Theory predict, and experiment confirms, that every elementary particle ${ }^{1}$ has an antiparticle of identical mass, identical lifetime, and of opposite signs of the electric charge and other discrete quantum properties. Some of them, such as the photon, $\gamma$, or the phi-meson, $\phi$ (which consists of a strange quark, $s$, and an anti-

\footnotetext{
${ }^{1}$ Not merely every truly elementary particle, such as the electron or the neutrino, but also any composite particle, such as the proton or any atomic nucleus.
} 
strange quark, $\bar{s}$ ) are truly neutral and thus are antiparticles of themselves-but in most cases the particle and the antiparticle are distinct.

For example, the neutron, $n$, and the antineutron, $\bar{n}$, are both electrically neutral, but are not at all identical: the neutron consists of three quarks, $u d d$, and, along with protons and electrons, is one of the three main building blocks of the physical world that surrounds us, while the antineutron consists of three antiquarks, $\bar{u} \bar{d}$, and can be found on Earth only in the cosmic rays that constantly bombard our planet, in products of interactions between cosmic rays and regular matter, and among the debris of subatomic particles produced by us, experimental particle physicists, at high-energy particle colliders.

\section{$1.4 C P T$ and $C P$}

The properties of quantum wave functions describing particles and fields under the action of the mathematical operators of charge conjugation $C$, spatial parity $P$, and time reversal $T$ play a very important role in elementary-particle physics. In particular, the CPT theorem, which applies to any "sane" quantum field theory (one that conserves energy, momentum, angular momentum, and does not allow events at one spatial point to immediately affect events at another), states that all predictions of the theory must remain invariant if the operators $C, P$, and $T$ are applied to its equations simultaneously. The equality of the masses and the lifetimes of a particle and an antiparticle is one of the most important consequence of the $C P T$ theorem. 
Until 1964, when Cronin and Fitch looked for and, quite unexpectedly, discovered an example to the contrary in the decays of neutral kaons [1], it was believed that the combined parity, $C P$, is also a precise symmetry of Nature. If $C P$ were a precise symmetry of Nature as well, a world built of antimatter would have behaved exactly like a mirror-like replica of a world built of matter. $C P$ violation gives rise to several interesting particle-physics phenomena, among which is the possible difference between the corresponding partial decay rates of a particle and an antiparticle, known as direct $C P$ violation: $C P$ violation allows the probability for a particle $X$ to decay into a final state $F$ to differ from the probability for its antiparticle (i.e., $C P$-conjugate) $\bar{X}$ to decay into the $C P$-conjugate final state $\bar{F}$.

Direct $C P$ violation was first observed in 1998 in neutral kaons [2, 3]; we at BABAR [45] are hard at work trying to discover more manifestations of this phenomenon. In the particular example of the rare decay of the charged $B$ meson $B^{ \pm} \rightarrow \phi K^{ \pm}$, which is one of the subjects of my dissertation research, direct $C P$ violation would reveal itself as a statistically significant difference between the numbers of observed $B^{+} \rightarrow \phi K^{+}$and $B^{-} \rightarrow \phi K^{-}$decays found in our immense amount of data (in $B A B A R$, the positively and negatively charged $B$ mesons are pair-produced, $e^{+} e^{-} \rightarrow \Upsilon(4 S) \rightarrow B^{+} B^{-}$, and so their numbers are guaranteed to be identical). 


\subsection{Creation and Annihilation of Particle-Antiparticle}

\section{Pairs}

Brought together, a pair of particles that consists of a particle and its antiparticle can annihilate each other, i.e., turn into nothing but two or more photons - light, or "energy", whatever you call it. For example, the nucleus of sodium-22 is unstable and, with a lifetime of 2.6 years, turns into neon-22 by emitting a positron (antielectron) and an electron neutrino. Soon after its creation, this positron will have a close encounter with an electron-perhaps, an electron that belonged to the very sodium- 22 atom that has just decayed - and annihilate with that electron, most likely into a pair of photons, each roughly of the energy $E_{\gamma}$ equal to $m_{e} c^{2} \approx 511 \mathrm{keV}$, where $m_{e}$ is the mass of the electron, $c$ is the speed of light, and $1 \mathrm{eV} \approx 1.6 \times 10^{-19} \mathrm{~J}$ is the unit of energy favored by particle physicists.

Likewise, a particle-antiparticle pair can be created in a collision of two sufficiently energetic photons. It is quite technologically challenging to produce such photons in the lab in the quantity and at the densities necessary to get interesting science done. Physicists are working on making a high-energy photon collider a reality; in the meantime, we achieve the same goal by making, accelerating and then smashing into each other electron-positron $\left(e^{+} e^{-}\right)$or proton-antiproton $(p \bar{p})$ beams. 


\subsection{The Big-Bang Connection}

Based on a variety of astrophysical observations (most importantly, the expansion of the Universe, its chemical_or, more precisely, isotopic - composition, and the existence of the cosmic microwave background, its temperature and slight non-uniformity) scientists believe that our Universe began some 12-15 billion years ago from a state of pure energy in a process called the Big Bang [4].

The very early Universe was very hot and very dense and was dominated by radiation; particle-antiparticle pairs were constantly created, mostly in photon-photon collisions, and destroyed through annihilation. So short was the average lifetime of these particles from the time of their creation to the time of their destruction that the Universe existed in a state of thermal equilibrium. The pair-creation mechanism and the equality of the particle and antiparticle masses dictated by the $C P T$ theorem imply that at this very early stage of Universe's existence the numbers of particles and antiparticles of each kind in the Universe was equal ${ }^{2}$; the Universe's matter-antimatter asymmetry, which we shall define as

$$
\mathcal{A}_{U}=\frac{\# \text { of particles }-\# \text { of antiparticles }}{\# \text { of particles }+ \text { \# of antiparticles }}
$$

was thus zero. From now on, let's pay attention only to baryons, i.e., particles made of quarks, such as protons and neutrons. The baryon asymmetry of the very early Universe

\footnotetext{
${ }^{2}$ up to tiny and irrelevant statistical fluctuations of the order of $\mathcal{O}\left(1 / \sqrt{n_{i}}\right), n_{i}$ being the number of particles of type $i$ in the Universe.
} 
was also zero.

The situation is very different in today's Universe: it is populated almost exclusively by matter; the tiny amounts of antimatter found in cosmic rays are due to collisions of energetic matter particles with the interstellar medium. Existence of macroscopic quantities of antimatter in the visible Universe (such as galaxies made of antimatter) is all but ruled out by astronomical observations.

This obviously implies that matter and antimatter are not exact "mirror" copies of each other: while virtualy all of the (baryonic) antimatter and almost all of the (baryonic) matter that existed in the early Universe were obliterated in the process of mutual matter-antimatter annihilation into photons, enough matter survived to form galaxies, nebulae, stars, planets — and us. To get a feeling of just how tiny this fraction of surviving matter is, take a look at the following number: in today's Universe, the ratio of the number of baryons to the number of photons is extremely small, $\eta \sim 5 \times 10^{-10}$.

\subsection{Sakharov's Three Conditions}

In his seminal 1967 article [5], Andrei Sakharov, then half-way from having been the father of the Soviet hydrogen bomb (first tested in 1953) to being awarded the 1975 Nobel Peace Prize, formulated the three conditions necessary for the creation of the observed baryon asymmetry of the Universe.

The first condition is a deviation at some suitable time in Universe's evolution from the thermal equilibrium that was discussed in the previous Section. An expanding Uni- 
verse is already slightly out of equilibrium, but this may not be enough. There are also other problems with the model of a steadily expanding Universe. It has therefore been proposed, and is now widely accepted among cosmologists, that early Universe underwent a period of extremely rapid inflation [6].

The second condition is non-conservation of the baryon number, i.e., existence of processes that change the value of $B=\#$ of baryons - \# of antibaryons in a closed system. No such processes have been observed so far and none are allowed by the Standard Model of elementary particles (which is extremely successful in describing all elementary-particle processes observed so far but is known to be internally inconsistent and thus incomplete). However, proposed extensions to the Standard Model easily allow processes that simultaneously violate $B$ and the lepton number $L$ while conserving $B-$ $L$; the decay of the proton into a positron and a pion, $p \rightarrow e^{+} \pi^{0}$, which has been extensively searched for [7], would be an example of such a process.

The third and the final of Sakharov's conditions for the creation of the baryon asymmetry of the Universe is the non-conservation of the $C$ and $C P$ parities in elementaryparticle interactions. While both are integral parts of the Standard Model, the amount of $C P$ violation allowed within its confines is far too small, by orders of magnitude, to account for the observed dominance of matter over antimatter in the cosmos. 


\subsection{Prospects}

At this point we can only hypothesize as to what the source of the "cosmological" $C P$ violation is. It is highly probable, but by no means guaranteed, that the ATLAS and CMS experiments at the Large Hadron Collider, currently under construction in Switzerland, will some time around 2010 uncover a whole new segment of elementary-particle phenomenology rich in new sources of $C P$ violation, such as that predicted by the theory of Supersymmetry — but chances are that that would not be enough either. However, every new result that comes from experimental elementary-particle physics and astrophysics helps theorists construct a better model of the conditions and events in the early Universe, conditions and events that cannot be recreated or even approached in a laboratory. In the decade that started in 1999, a good chunk of this new knowledge is coming from two B-meson "factories", PEP-II at the Stanford Linear Accelerator Center and KEKB in Tsukuba, Japan, which were built specifically to study $C P$ violation in samples of hundreds of millions of $B$-mesons, the best test subjects the Standard Model provides us with for this purpose. In particular, it is hoped that the high-precision measurements made at the $B$-factory detectors, $B A B A R$ at PEP-II and Belle at KEKB will be able to experimentally "crack" the Standard Model before ATLAS and CMS start taking data. 


\section{Chapter 2}

\section{Theoretical Introduction}

\section{1 $C P$ violation in the Standard Model}

Within the Standard Model of particle physics [11], the only ${ }^{1}$ source of $C P$ violation is the complex parameter of the unitary $3 \times 3$ Cabibbo-Kobayashi-Maskawa (CKM) quark-mixing matrix $V_{\mathrm{CKM}}$ [14], which, by convention, relates the mass (flavor) eigenstates of the $d, s$, and $b$ quarks to the electroweak-interaction eigenstates $d^{\prime}, s^{\prime}$, and $b^{\prime}:$

${ }^{1}$ The discussion of "the strong $C P$ problem" [15], another potential source of $C P$ violation in the Standard Model, the unnaturally small [16] (or absent $[17,18]$ ) $P$ - and $T$-violating term in the Lagrangian of strong interaction that is proportional to the trace of the square of the gluon field-stress tensor, $\mathcal{L}_{\theta}=$ $\theta \frac{g_{\mathrm{s}}^{2}}{32 \pi^{2}} F_{\mu \nu}^{a} \tilde{F}_{a \mu \nu}$, is beyond the scope of this dissertation. 


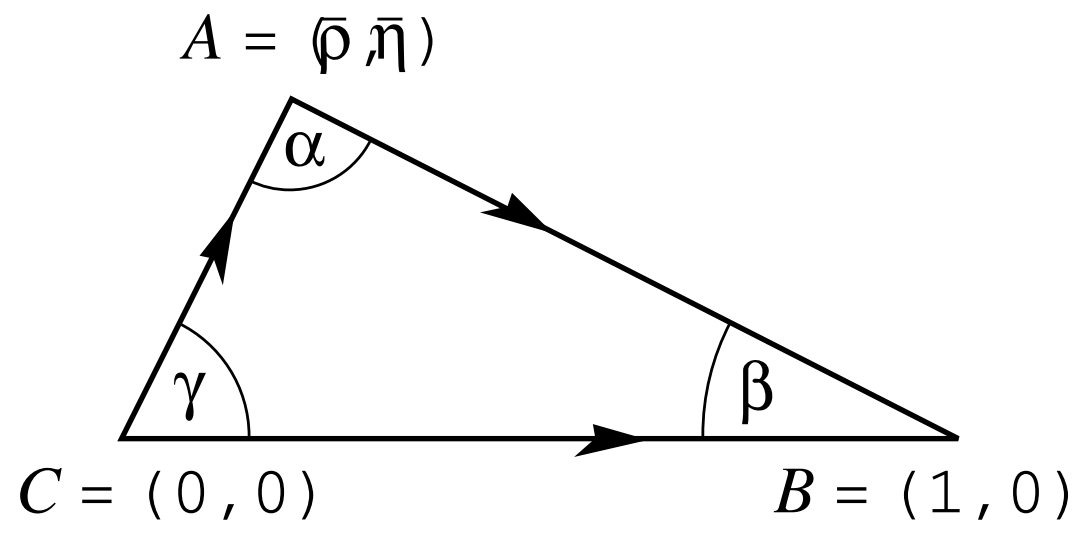

Figure 2.1: Graphical representation of Eq. 2.3 in the form of a triangle on the complex plane. The triangle is rescaled so that $C=(0,0)$ and $B=(1,0)$; then $A=(\bar{\rho}, \bar{\eta})$ (Eq. 2.4).

$$
\left(\begin{array}{l}
d^{\prime} \\
s^{\prime} \\
b^{\prime}
\end{array}\right)=\left(\begin{array}{ccc}
V_{u d} & V_{u s} & V_{u b} \\
V_{c d} & V_{c s} & V_{c b} \\
V_{t d} & V_{t s} & V_{t b}
\end{array}\right)\left(\begin{array}{l}
d \\
s \\
b
\end{array}\right)
$$

Following the convenient approximation proposed by Wolfenstein [19],

$$
V_{\mathrm{CKM}}=\left(\begin{array}{ccc}
1-\lambda^{2} & \lambda & A \lambda^{3}(\rho-i \eta) \\
-\lambda & 1-\lambda^{2} & A \lambda^{2} \\
A \lambda^{3}(1-\rho-i \eta) & -A \lambda^{2} & 1
\end{array}\right)+\mathcal{O}\left(\lambda^{4}\right)
$$

where $\lambda, A, \rho$, and $\eta$ are real parameters, one can express the unitarity relationship between the first and the third columns of the CKM matrix,

$$
V_{u d} V_{u b}^{*}+V_{c d} V_{c b}^{*}+V_{t d} V_{t b}^{*}=0
$$

graphically in the form of a "unitarity triangle" [12], in which, after rescaling so that 
the base becomes of unit length (Fig. 2.1), the coordinate of the apex $A$ on the complex plane becomes

$$
A=\frac{\Re\left(V_{u d} V_{u b}^{*}\right)}{\left|V_{c d} V_{c b}^{*}\right|}+\imath \frac{\Im\left(V_{u d} V_{u b}^{*}\right)}{\left|V_{c d} V_{c b}^{*}\right|} \approx \rho\left(1-\lambda^{2} / 2\right)+\imath \eta\left(1-\lambda^{2} / 2\right) \equiv \bar{\rho}+\imath \bar{\eta} .
$$

The angles $\alpha, \beta$, and $\gamma$ of the unitarity triangle, which can be written in terms of elements of the CKM matrix,

$$
\alpha=-\arg \frac{V_{t d} V_{t b}^{*}}{V_{u d} V_{u b}^{*}}, \beta=-\arg \frac{V_{c d} V_{c b}^{*}}{V_{t d} V_{t b}^{*}}, \gamma=-\arg \frac{V_{u d} V_{u b}^{*}}{V_{c d} V_{c b}^{*}},
$$

can be experimentally measured in decays of the $B^{0}, B^{+}$, and $B_{s}{ }^{2}$ mesons to specific final states [44, 13]; a deviation from $\alpha+\beta+\gamma=180^{\circ}$ or failure of measurements of the sides of the triangle [12] to agree with the measurements of its angles would be an indication of physics beyond the Standard Model. $C P$ violation in $B$ mesons has been established by the high-precision measurements of the angle $\beta$ in the tree-diagram-dominated charmonium-neutral-kaon ("golden") modes $B^{0} \rightarrow J / \psi K_{S}^{0}, J / \psi K_{L}^{0}, \psi(2 S) K_{S}^{0}, \eta_{c} K_{S}^{0}$, $\chi_{c 1} K_{S}^{0}$, and $J / \psi K^{* 0}\left(K^{* 0} \rightarrow K_{S}^{0} \pi^{0}\right)$ by BABAR [20] and Belle [21]; the currently available measurements of the angles $\alpha$ and $\gamma$ are yet of limited precision and suffer from theoretical uncertainties.

We present in Fig. 2.2 [22] current experimental constraints on the coordinates of the apex $A=(\bar{\rho}, \bar{\eta})$ of the unitarity triangle obtained within the framework of the Standard Model by the CKMfitter Working Group using the methodology described in [23].

\footnotetext{
${ }^{2}$ Not accessible at existing $B$-meson factories.
} 


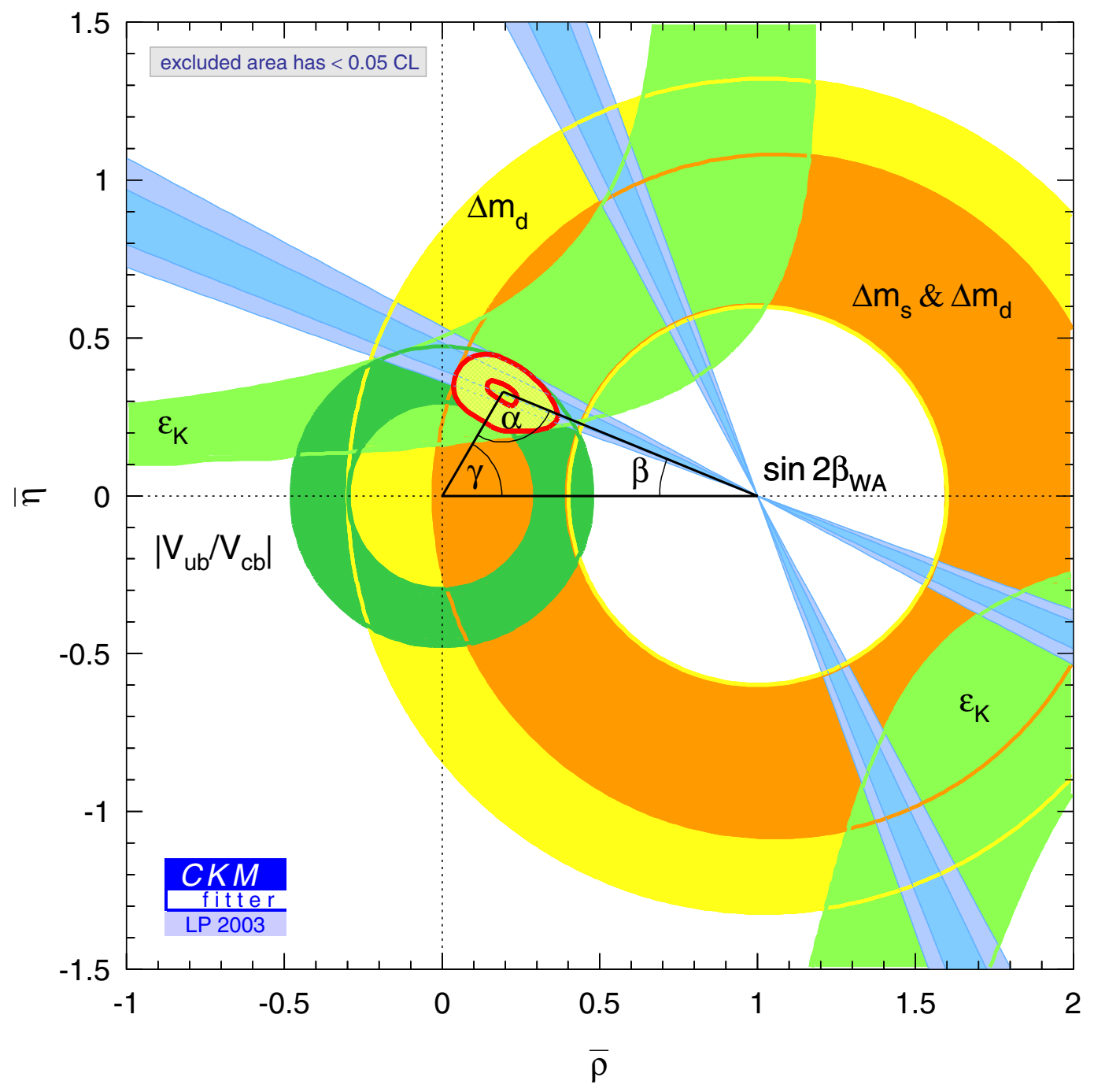

Figure 2.2: Current experimental constraints on the apex $A=(\bar{\rho}, \bar{\eta})$ of the unitarity triangle $[22,23,24]$. $C P$-violating quantities measured in $B$-meson decays are not used in the fit; the charmonium-neutral-kaon $\sin 2 \beta$ world average (the $1 \sigma$ and $2 \sigma$ cones passing through the origin) is overlaid with the Standard Model expectation ( $1 \sigma$ and $2 \sigma$ contours are shown). 


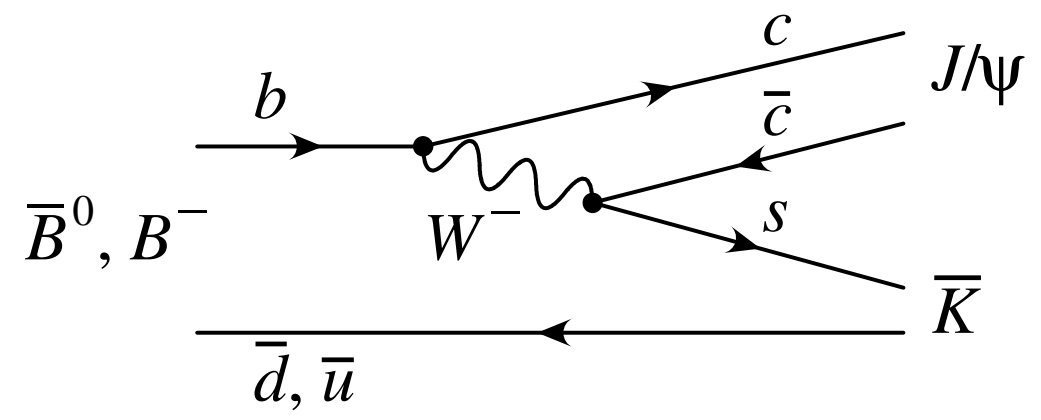

Figure 2.3: Example of a tree-level diagram: $B \rightarrow J / \psi K$.

The list of references for the inputs to the fit is available at [24]. $C P$-violating quantities measured in $B$-meson decays are not used in the fit; the charmonium-neutral-kaon $\sin 2 \beta$ world average is overlaid with the Standard Model expectation. The agreement of the SM-based prediction with the "golden"-mode $\sin 2 \beta$ measurements is quite remarkable.

\subsection{Pure-penguin $B$-meson decays: a window to physics beyond the Standard Model}

Quasi-two-body charmless decays of $B$ mesons that contain a $\phi$ resonance in the final state have recently been at the focus of a great many theory papers that ponder the possibility of discovering effects of physics beyond the Standard Model in such decays.

What sets these decays apart from the majority of experimentally accessible $B$ meson decay modes is the fact that they are dominated not by tree-level processes (Fig. 2.3) but by $b \rightarrow s(d) \bar{s} s$ gluonic loop-level (“penguin”) diagrams (Fig. 2.4), possi- 


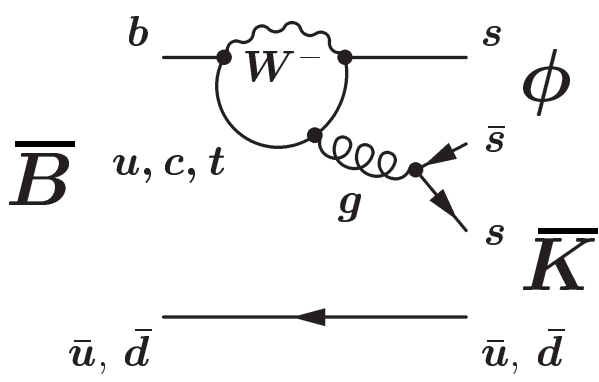

(a)

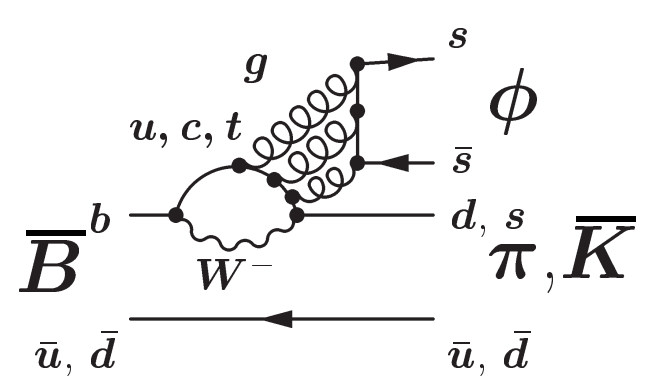

(b)

Figure 2.4: Examples of quark-level diagrams for $B \rightarrow \phi K$ and $B \rightarrow \phi \pi:(a)$ internal gluonic penguin; $(b)$ flavor-singlet gluonic penguin.

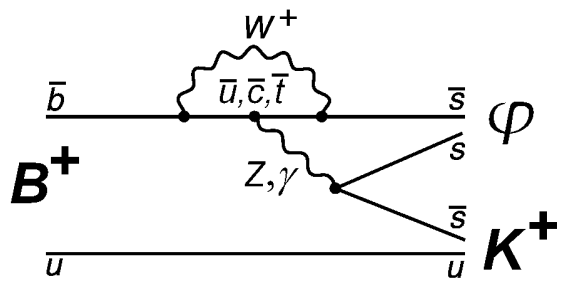

(a)

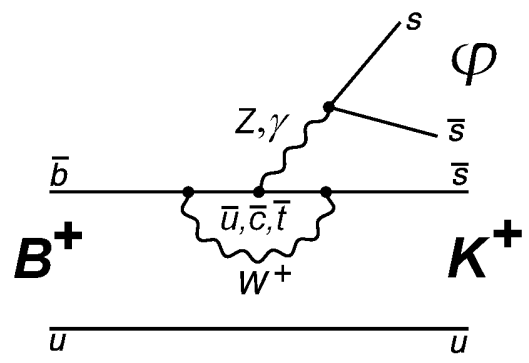

(b)

Figure 2.5: Examples of quark-level diagrams for $B \rightarrow \phi K$ and $B \rightarrow \phi \pi:(a)$ internal electroweak penguin for $B^{ \pm} \rightarrow \phi K^{ \pm} ;(b)$ external electroweak penguin for $B^{ \pm} \rightarrow \phi K^{ \pm}$.

bly with smaller but non-negligible contribution from electroweak penguins (Fig. 2.5), while other Standard Model contributions are strongly suppressed [25, 26, 27]. Since many scenarios of physics beyond the Standard Model introduce additional loop diagrams with heavy particles and new $C P$-violating phases $[28,29]$, comparison of $C P$ violating observables in $B \rightarrow \phi K$ with Standard Model expectations offers a sensitive probe for new physics. 
In the Standard Model, the direct $C P$ violation in $B^{+} \rightarrow \phi K^{+3}$, detected as an asymmetry $\mathcal{A}_{C P}=\left(\Gamma_{\phi K^{-}}-\Gamma_{\phi K^{+}}\right) /\left(\Gamma_{\phi K^{-}}+\Gamma_{\phi K^{+}}\right)$in the decay rates $\Gamma_{\phi K^{ \pm}}=\Gamma\left(B^{ \pm} \rightarrow\right.$ $\left.\phi K^{ \pm}\right)$, is expected to be $\lesssim 1 \%$; in the presence of large new-physics contributions to the $b \rightarrow s \bar{s} s$ transition, it could be of order $1[30,31,32,33,34]$. Current experimental sensitivity to $\mathcal{A}_{C P}\left(B^{ \pm} \rightarrow \phi K^{ \pm}\right)$at both $B A B A R$ and Belle is $\sim 10 \%$, which, if statistically consistent with the SM prediction, would exclude a substantial part of the parameter space of some of the existing new-physics models while still leaving plenty room for others-or, if not consistent with zero, would unambiguously signal a breakdown of the Standard Model.

Recent intriguing preliminary results from $B A B A R$ and Belle on the time-dependent $C P$ asymmetry in the decay $B^{0} \rightarrow \phi K_{S}^{0}[76,77]$ have raised questions among some theorists about the magnitude of the difficult-to-compute $b \rightarrow u W^{-}(\bar{u} s), u \bar{u} \rightarrow s \bar{s}$ rescattering contribution to the $B^{0} \rightarrow \phi K^{0}$ decay amplitude (Fig. 2.6). If the rescattering amplitude is indeed significant in $B^{0} \rightarrow \phi K^{0}$, it should also be significant—and, in fact, larger by a factor of $\cot \left(\theta_{\text {Cabibbo }}\right) \approx 4.4-$ in $B^{ \pm} \rightarrow \phi \pi^{ \pm}$. By establishing an upper limit on $\mathcal{B}\left(B^{ \pm} \rightarrow \phi \pi^{ \pm}\right)$, our analysis quite stringently constrains the magnitude of the rescattering contribution to $B^{0} \rightarrow \phi K^{0}$.

Speculations about the rescattering amplitude aside, the $B \rightarrow \phi \pi$ decay rate is sensitive to new physics as well: it is strongly color- and CKM-suppressed in the $\mathrm{SM}$ [35], and a measurement of $\mathcal{B}(B \rightarrow \phi \pi) \gtrsim 10^{-7}$ would serve as evidence for new physics [36].

\footnotetext{
${ }^{3}$ Charge-conjugate states included unless explicitly stated otherwise.
} 


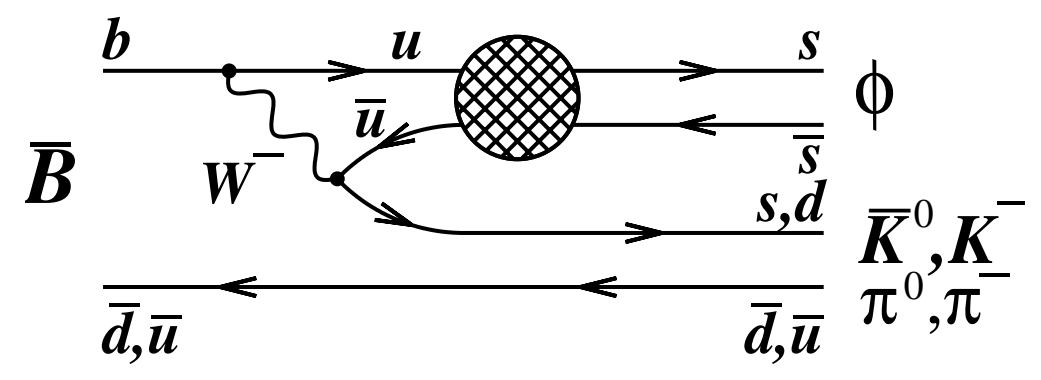

Figure 2.6: The rescattering diagram for $B \rightarrow \phi K$ and $B \rightarrow \phi \pi$. The tree part of this diagram could lead directly to a $B \rightarrow \phi h$ final state via the small, poorly understood $u \bar{u}$ component of the $\phi$ resonance.

Additional reasons to be interested in a detailed study of the $b \rightarrow s(d) \bar{s} s$ processes include their sensitivity to QCD dynamics $[37,38]$ and to the poorly measured CabibboKobayashi-Maskawa matrix element $V_{t s}$.

The two decay modes studied in the present version of this BAD note, $B^{ \pm} \rightarrow \phi K^{ \pm}$ and $B^{0} \rightarrow \phi K_{S}^{0}$, have previously been observed by CLEO, BABAR, and Belle [39, 40, 41]; $\mathcal{A}_{C P}\left(B^{ \pm} \rightarrow \phi K^{ \pm}\right)$has been studied by $B A B A R$ [42]. Thanks to the greatly increased size of the $B A B A R$ data set and an improved analysis technique, we are able, compared to the previous analyses, to significantly reduce both the statistical and the systematic errors on both the branching fractions and the charge asymmetry.

The analysis is based on a multivariate unbinned extended-maximum-likelihood fit to the signal and the background; the decay modes $B^{ \pm} \rightarrow \phi K^{ \pm}$and $B^{ \pm} \rightarrow \phi \pi^{ \pm}$are fitted for simultaneously. A blind analysis technique is used throughout to avoid the potential for an experimenter-induced bias: the signal region is hidden until all analysis details, with the exception of completing determination of systematic errors, are 
finalized. 


\section{Chapter 3}

\section{Instrumentation and the Data Set}

\subsection{The PEP-II $B$-meson factory}

The PEP-II [43] is a modern high-luminosity two-ring asymmetric $e^{+} e^{-}$collider at the Stanford Linear Accelerator Center (SLAC) that uses SLAC's three-kilometer linearaccelerator complex as the injector (Fig. 3.1). Electron and positron beams of the energies 9.0 GeV and 3.1 GeV, respectively, are collided head-on, which is made possible by a rather unconventional design of the interaction region (Fig. 3.2).

PEP-II usually operates at the peak of the $\Upsilon(4 S)$ resonance (center-of-mass energy $\sqrt{s}=10.580 \mathrm{GeV}$ ), which decays nearly $100 \%$ of the time into a pair of $B$ mesons, $B^{0} \bar{B}^{0}$ or $B^{+} B^{-}$; approximately $12 \%$ of data is taken $40 \mathrm{MeV}$ below the $\Upsilon(4 S)$, or approximately $20 \mathrm{MeV}$ below the $B \bar{B}$ production threshold, for use in studies of combinatorial backgrounds originating from continuum quark- and tau-pair production and from two-photon events (Fig. 3.3). Since the PEP-II final-focus system relies on per- 


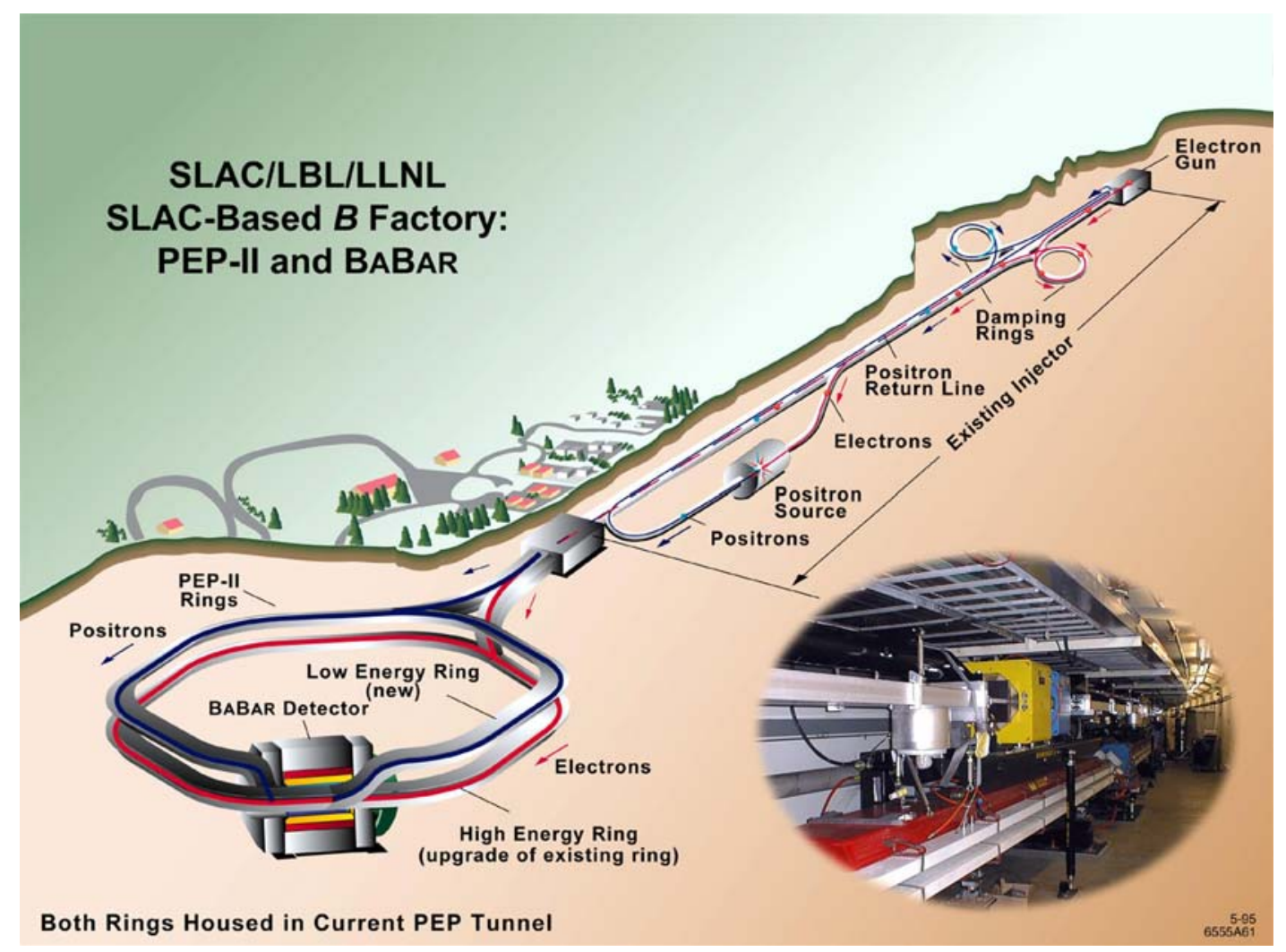

Figure 3.1: Schematic drawing of the PEP-II $e^{+} e^{-}$storage rings and the SLAC three-kilometer linear-accelerator complex, which serves as the PEP-II injector. 


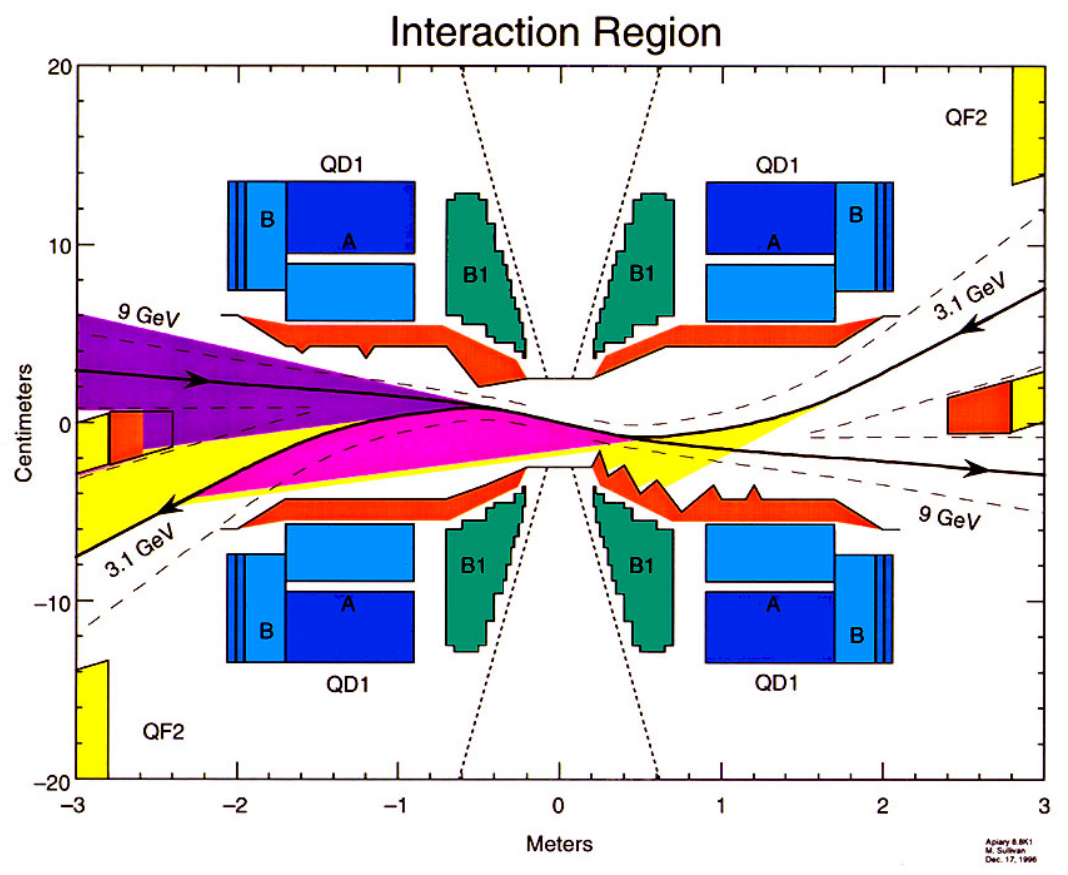

Figure 3.2: Drawing of the PEP-II interaction region in the horizontal plane. Shown are the magnets (quadrupoles QD1 and QF2 and the dipole B1-note that the magnet closest to the interaction point is a dipole), synchrotron-radiation fans produced as the beams are bent, and synchrotron-radiation-blocking masks. The beams are collided head-on; at the interaction point, the beams are tilted by $20 \mathrm{mrad}$ with respect to the $Z$ axis of the $B A B A R$ detector.

manent magnets, its center-of-mass energy is adjustable only within a relatively narrow window: the $\Upsilon(5 S)$, or $\Upsilon(10860)$, resonance, which would make the $B_{s}$ meson available to $B A B A R$, cannot be reached without a major upgrade to the PEP-II interaction region; however, the $\Upsilon(3 S)$ resonance can and has been produced at PEP-II without a noticeable degradation in luminosity.

The asymmetry in the energies of the electron and the positron beams provides a boost of $\beta \gamma \approx 0.56$ in the laboratory frame of reference to the $\Upsilon(4 S)$ and its decay products, which significantly enhances the $B$-meson flight length and makes possible 


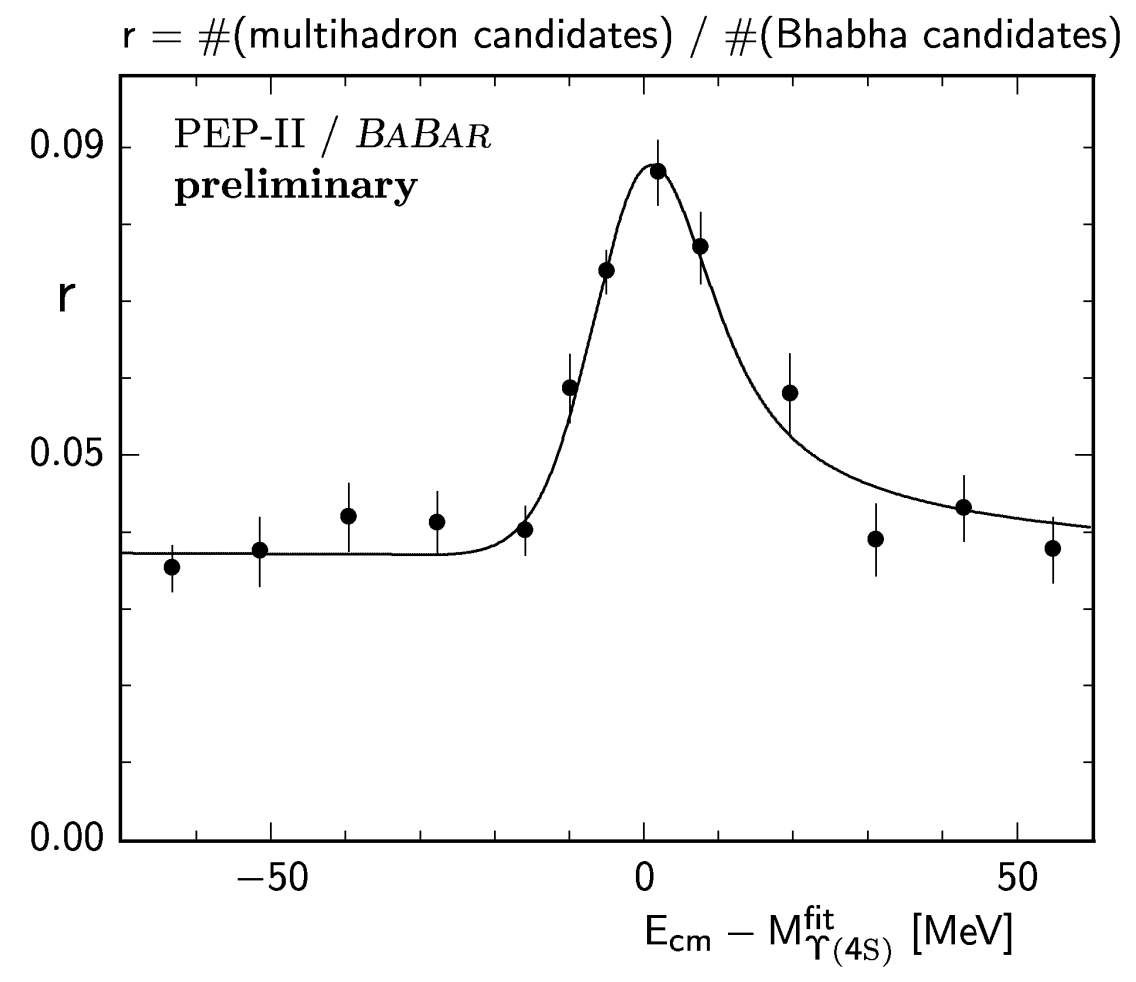

Figure 3.3: A PEP-II scan of the $\Upsilon(4 S)$ resonance. At the peak of the $\Upsilon(4 S)$ resonance, $\sigma\left(e^{+} e^{-} \rightarrow B \bar{B}\right)=1.0854 \pm 0.0003$ (stat.) \pm 0.0238 (syst.) nb [81]. Other relevant cross sections at the $\Upsilon(4 S)$ peak are, approximately, $\sigma(d \bar{d})=0.35 \mathrm{nb}, \sigma(u \bar{u})=1.39 \mathrm{nb}, \sigma(s \bar{s})=$ $0.35 \mathrm{nb}, \sigma(c \bar{c})=1.30 \mathrm{nb}, \sigma\left(\tau^{+} \tau^{-}\right)=0.94 \mathrm{nb}, \sigma\left(\mu^{+} \mu^{-}\right)=1.16 \mathrm{nb}$, and $\sigma\left(e^{+} e^{-}\right) \approx 40 \mathrm{nb}$ (within the tracking acceptance of $B A B A R$ ) [44]. 
determination of the distance $\Delta z$ between the decay vertices of the two $B$ mesons with a silicon vertex detector, thus enabling the time-dependent $C P$ asymmetry measurements such as [20].

Construction of the PEP-II, a joint project between SLAC (the electron ring) and the Lawrence Berkeley and Lawrence Livermore National Laboratories (the positron ring) was started in early 1994; first collisions took place in July 1998, and colliding beams were delivered to BABAR in May 1999. For over two years, PEP-II was the highestluminosity collider in the world; the design peak luminosity, $3.0 \times 10^{33} \mathrm{~cm}^{-2} \mathrm{~s}^{-1}$, was reached in October 2000, and, as of November 14, 2003, the record peak luminosity is $6.6 \times 10^{33} \mathrm{~cm}^{-2} \mathrm{~s}^{-1}$, or the design $\times 2.2$.

At the design stage of both PEP-II and KEKB, it was thought that the adverse effect of beam-beam interactions on luminosity would be minimized if $I_{e^{-}} \times E_{e^{-}}=I_{e^{+}} \times E_{e^{+}}$. This prediction turned out to be inaccurate: the latest PEP-II peak-luminosity record was set with stored beam currents of $I_{e^{-}}=1.33 \mathrm{~A}$ and $I_{e^{+}}=1.94 \mathrm{~A}\left(\mathrm{design}: I_{e^{-}}=0.7 \mathrm{~A}\right.$, $I_{e^{+}}=2.1 \mathrm{~A}$ ) and 1230 colliding bunches (design: 1658); an upgrade to the RF-power systems in both the electron and the positron rings was needed to achieve the higherthan-design currents.

A total of $149 \mathrm{fb}^{-1}$ has been delivered by PEP-II, and $142 \mathrm{fb}^{-1}$ recorded by BABAR (Fig. 3.4) since October 1999. 


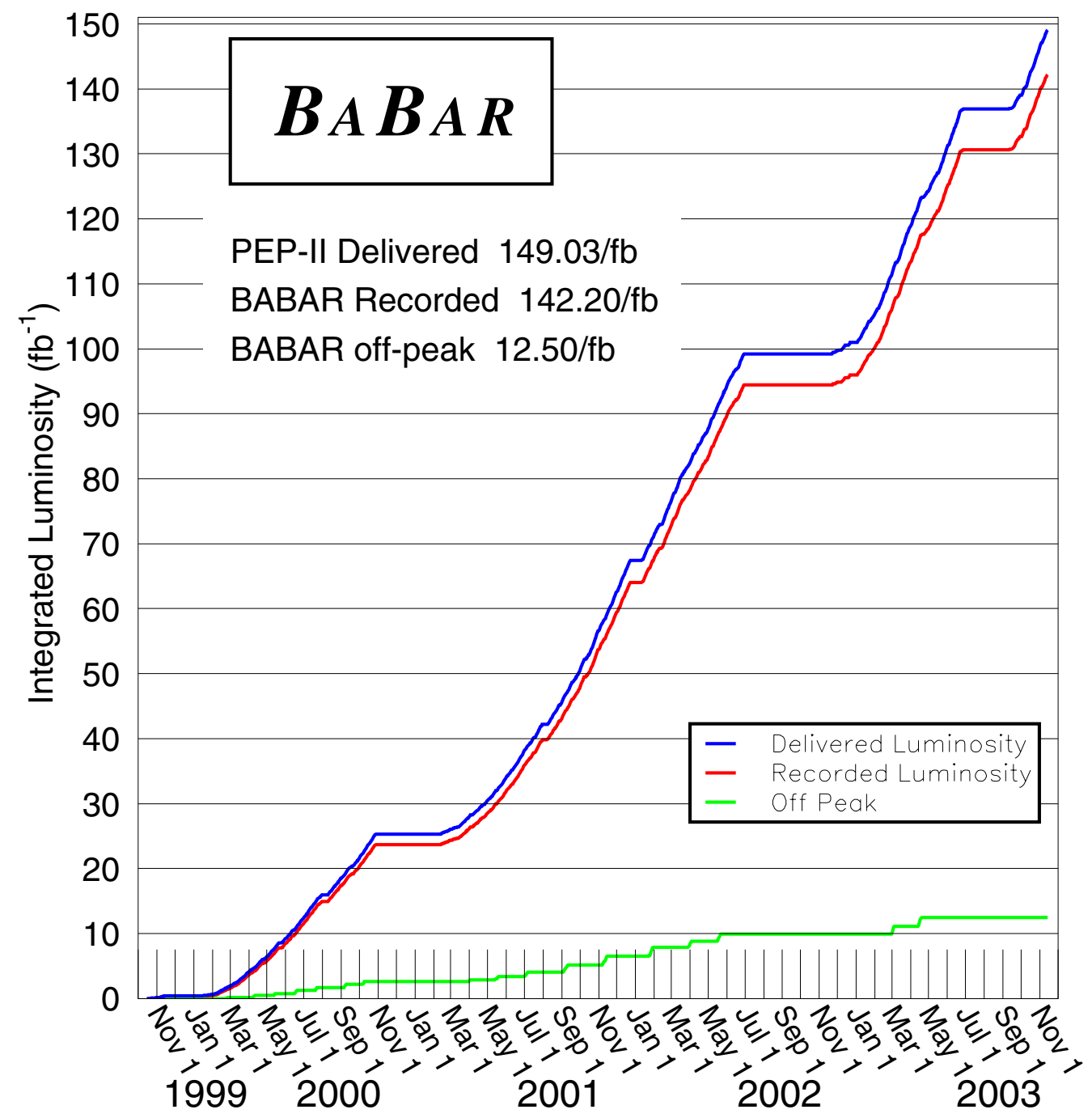

Figure 3.4: PEP-II-delivered and BABAR-recorded luminosities integrated since October 1999. 


\subsection{Overview of the $B_{A} B_{A R}$ detector}

While having many features in common with other collider-based high-energy-physics detectors, in order to achieve its ambitious goals, the BABAR detector [45] utilizes some of the most modern detector technologies available in mid-1990s.

Moreover, the unprecedentently high data-processing and data-storage requirements of the $B A B A R$ experiment could not be met by the computer hardware and software technologies available when the experiment was approved for construction in 1994, and so the success of the experiment has and will continue to depend on further advances in these technologies.

Specific requirements to a $B$-factory detector include the following:

- Excellent vertexing resolution to satisfy the requirements of the time-dependent $C P$-violation analyses - achieved by the Silicon Vertex Tracker $(\mathrm{SVT})^{1}$;

- Excellent charged-particle flavor identification to meet the needs of a wide variety of analyses, including the time-dependent $C P$-violation analyses and the charmless direct- $C P$ analyses that are discussed in the physics-analysis section of this dissertation-achieved by the DIRC ("Detection of Internally Reflected Cherenkov light”), a novel fused-silica ring-imaging Cherenkov detector, with the help of the ionization-energy-loss $(\mathrm{d} E / \mathrm{d} x)$ information provided by the Drift Chamber and the SVT;

\footnotetext{
${ }^{1}$ For the reader's convenience, a glossary of terms related to $B_{A} B_{A}$ is provided in Appeindix A. Terminology that is specific or particularly important to the topic of this dissertation is introduced in Chapter 4 .
} 


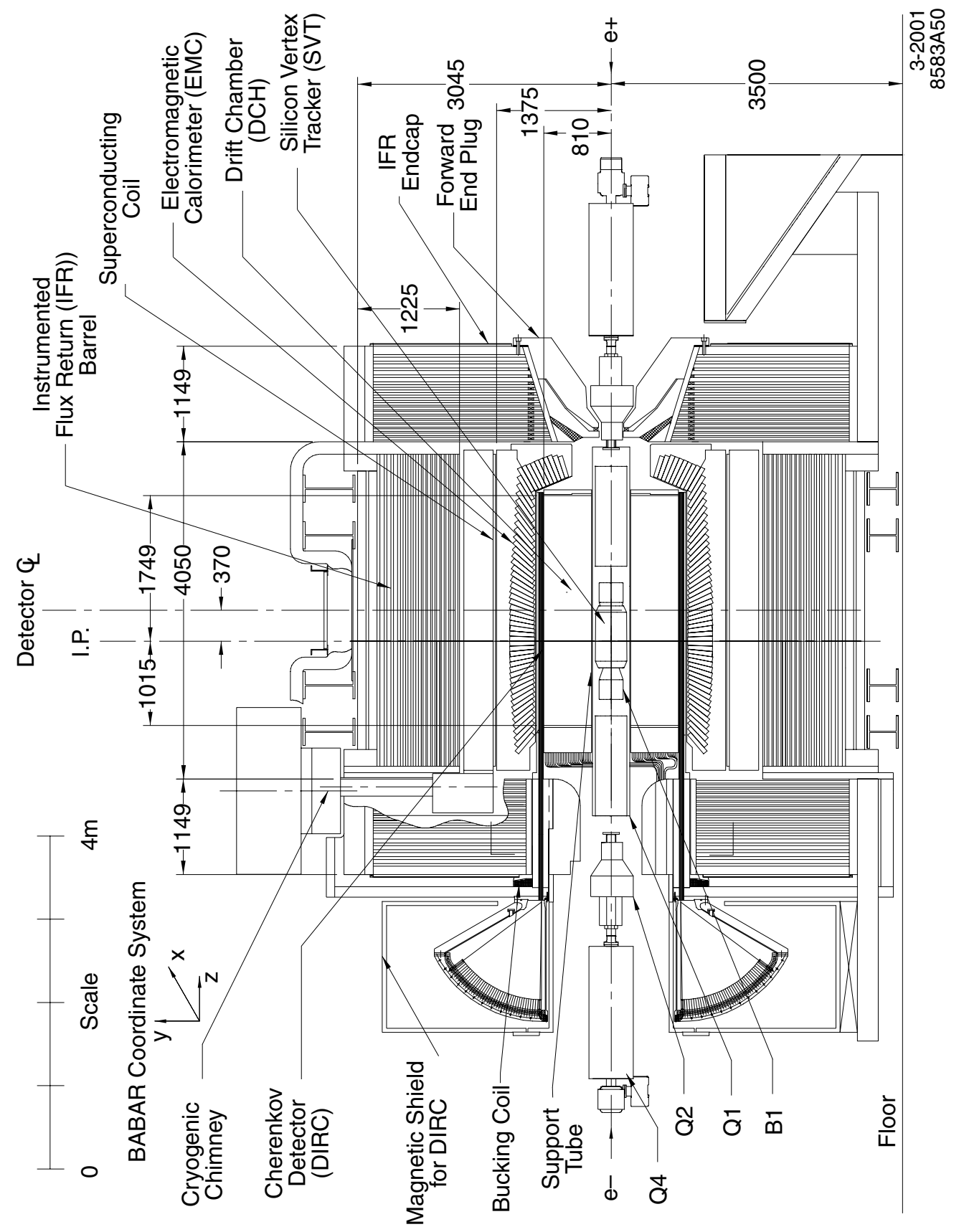

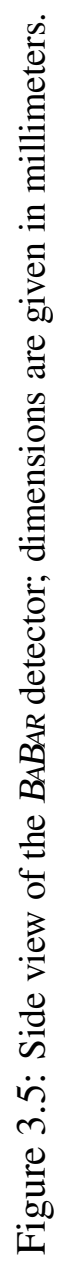




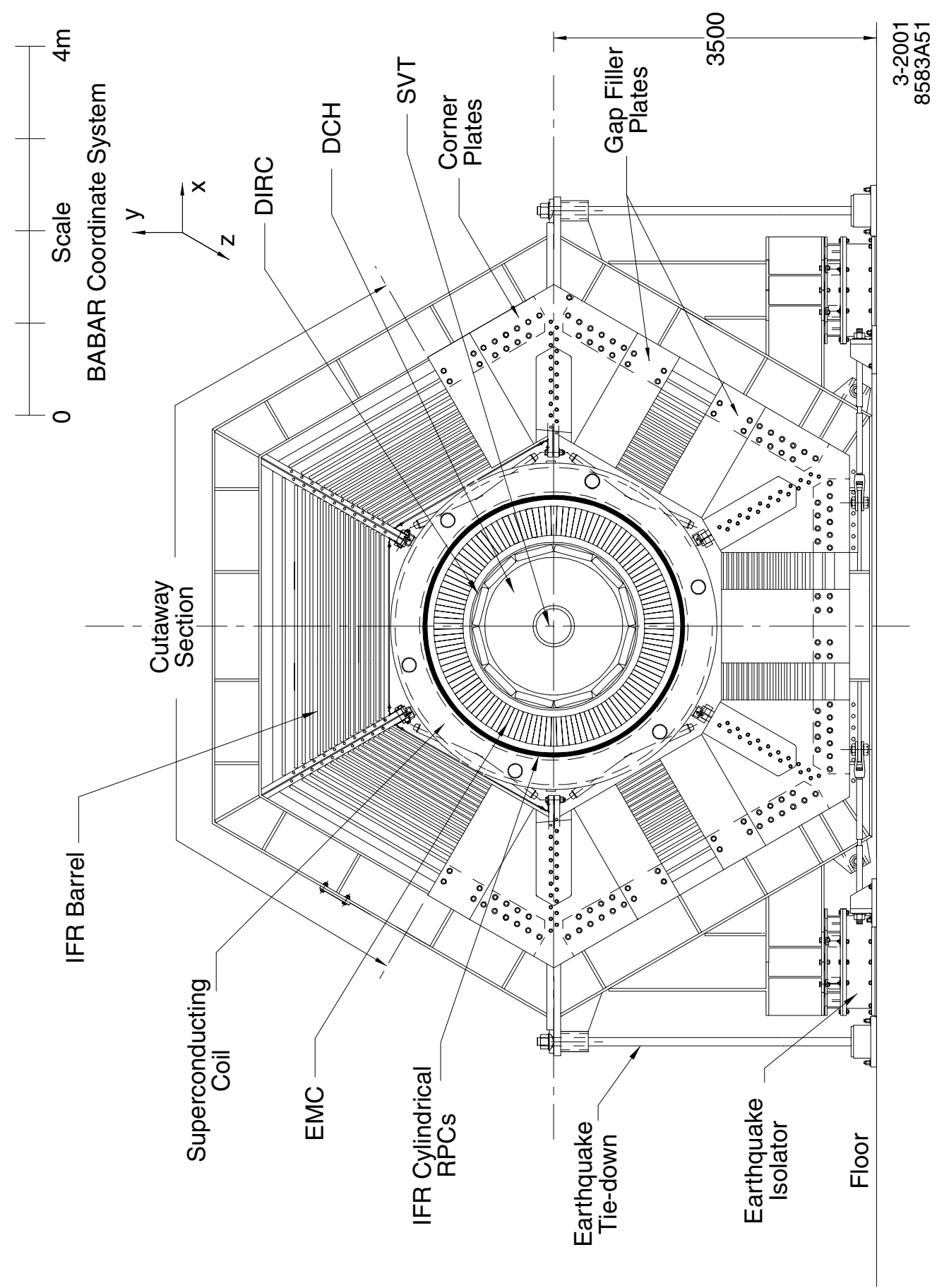

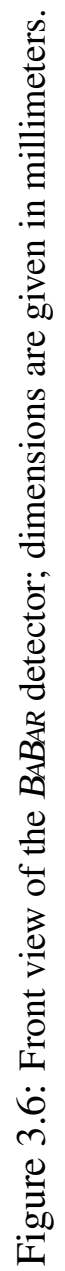


- Highly efficient and accurate charged- and neutral-particle reconstruction, achieved, respectively, by the high-acceptance Drift Chamber (DCH) with the help of the SVT, and by the Electromagnetic Calorimeter (EMC);

- Ability of vital detector components to survive for many years in the high-radiation environment of PEP-II.

Figs. 3.5 and $3.6^{2}$ show the side- and front-view cross section schematics of the $B A B A R$ detector. Nested inside the $1.5 \mathrm{~T}$ magnetic field created by a superconducting niobium-titanium solenoid are the Silicon Vertex Tracker, the Drift Chamber, the DIRC, and the Electromagnetic Calorimeter. Outside the solenoid is the Instrumented Flux Return, which provides muon and $K_{L}^{0}$ identification. Detailed information on each of the $B A B A R$ subdetectors is provided in the following five sections.

\subsection{Vertexing}

The BABAR Silicon Vertex Tracker [46] (Figs. 3.7, 3.8), together with the Drift Chamber a part of the BABAR tracking system, provides efficient detection of charged particles and helps in high-precision determination of their momenta and directions of flight. More importantly, the SVT, expecially its three inner layers, is most critical for the measurement of time-dependent $C P$ asymmetries. Indeed, time-dependent $C P$-violation measurements in the $B \bar{B}$ system rest on the ability of the vertex detector to measure the separation $\Delta z$ between the decay vertices of the two $B$ mesons with a precision better

\footnotetext{
${ }^{2}$ These and most other figures in this Chapter have previously been published in [45].
} 
than $1 / 2$ of the mean separation (which at $B A B A R$ is approximately $260 \mu \mathrm{m}$ ). That is, the mean vertex resolution along the $Z$ axis for a fully reconstructed $B$-meson decay is required to be better than $80 \mu \mathrm{m}$. Similarly, the study of $B \rightarrow D \bar{D}$ decays requires at least $\mathrm{a} \sim 100 \mu \mathrm{m}$ vertex resolution in the $X Y$ plane. These resolutions are easily achievable with the silicon micro-strip technology that had been chosen for the BABAR vertex detector.

The SVT is built of 340 double-sided silicon modules, which amounts to $0.96 \mathrm{~m}^{2}$ of active detector-surface area; the geometrical acceptance of the SVT is $90 \%$ of solid angle in the PEP-II center-of-mass frame. The direction of silicon strips on one side of each module is perpendicular to that on the other side, so that each module provides both the $z$ and $\phi$ coordinates of a charged-particle track; the total number of readout channels is close to 150,000 .

The distance between readout strips varies from $50 \mu \mathrm{m}$ to $210 \mu \mathrm{m}$, which leads to a single-hit resolution of $15 \mu \mathrm{m}$ to $50 \mu \mathrm{m}$ in $z$ and $11 \mu \mathrm{m}$ to $35 \mu \mathrm{m}$ in $\phi$, depending on the layer (better for innermost layers) and the incident angle of the track. The typical resolution on the $Z$ coordinate of the decay vertex of a fully reconstructed $B$ meson is $70 \mu \mathrm{m}$; the r.m.s. resolution on the separation $\Delta z$ between the fully reconstructed and the tag $B$ mesons is $180 \mu \mathrm{m}$.

A process of individually calibrating positions of each of the 340 silicon modules, known as the "local alignment", mostly relies on $e^{+} e^{-} \rightarrow \mu^{+} \mu^{-}$tracks and is performed once every few weeks, typically after each detector access. A "global alignment" procedure, which is necessary to account for movement of the SVT as a whole as a result 


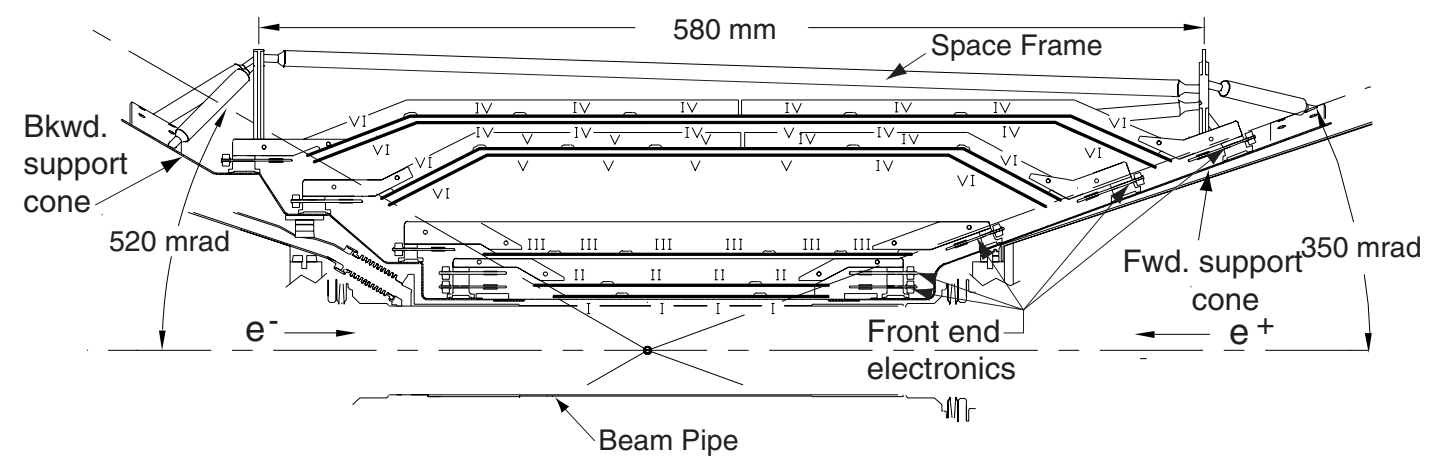

Figure 3.7: Schematic view of the $B A B A R$ Silicon Vertex Tracker. The Roman numerals label six different types of sensors. The radius of the PEP-II beam pipe is $27.8 \mathrm{~mm}$; the radii of SVT's five layers are, respectively, $32 \mathrm{~mm}, 40 \mathrm{~mm}, 54 \mathrm{~mm}, 91-127 \mathrm{~mm}$, and 114-144 mm.

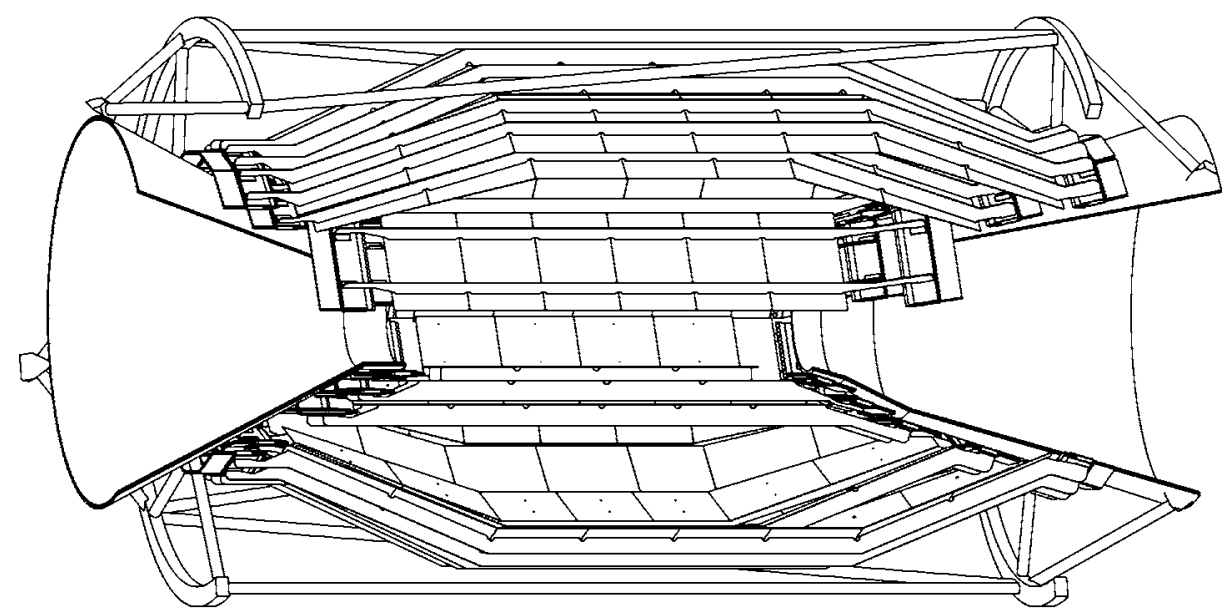

Figure 3.8: A cutout view of the Silicon Vertex Tracker. Shown are the double-sided silicon strip sensors and the support structure: two carbon-fiber cones connected by a "space frame" made of carbon-fiber epoxy laminate. 
of temperature variations and tides, is performed several times each day.

The SVT utilizes a radiation-hard technology and was designed to withstand a radiation dose of $2 \mathrm{MRad}$, the equivalent of 10 years in radiation environment of PEP-II originally expected during operation at the design luminosity. Recent tests have shown that the SVT can actually withstand a dose of 10 MRad without a significant degradation in resolution and efficiency. However, the PEP-II average luminosity has already surpassed the design value by a factor of $\sim 2.5$; additionally, the radiation near the PEP-II beam pipe, most of it originating from lost beam particles and from synchrotron radiation, has exceeded the originally predicted level. As a result, SVT modules lying in the plane of the PEP-II rings will be replaced with spares during the Summer 2005 shutdown. If necessary, the life span of the SVT can be extended further without the need to manufacture additional modules by swapping the hardest-hit modules with those away from the horizontal plane of the detector.

\subsection{Tracking}

In $B A B A R$, the trajectories of charged particles are reconstructed from the ionization trails that they leave in the active volume of the Drift Chamber and from their interactions with the detector components of the SVT. As in most other collider-based experiments, the momenta of charged particles are determined by measuring the curvature of their trajectories as they traverse an area of solenoidal magnetic field, i.e., magnetic field that is parallel to the direction of the beams; in $B A B A R$, the $1.5 \mathrm{~T}$ field is nearly uniform, with 
deviations from the mean of no more than $2.5 \%$ within the entire tracking volume.

The unusually large inner radius of the BABAR Drift Chamber, $23.6 \mathrm{~cm}$, was dictated by the design needs of the PEP-II final-focus system (Figs. 3.2, 3.5). As a result, the DCH alone cannot provide reliable tracking for charged particles with transverse momentum less than $120 \mathrm{MeV} / c$. Since in the study of $D^{*}$ decays the ability to track the slow pions is crucial, the fourth and the fifth layers were added to the design of the vertex detector; while not contributing much to vertexing, these layers enable the SVT to provide standalone tracking for particles with $p_{T}$ in the $50-120 \mathrm{MeV} / c$ range. For higher-momentum tracks, $B A B A R$ tracking is dominated by the $\mathrm{DCH}$.

The DCH [47] is a 3-meter-long cylindrical proportional wire chamber with the inner radius of $23.6 \mathrm{~cm}$ and the outer radius of $80.9 \mathrm{~cm}$ (Fig. 3.9) that contains 7104 small hexagonal drift cells in 40 layers organized in ten superlayers (four axial and six stereo) of four layers each (Figs. 3.10, 3.11). Charged tracks emitted from the IP within the polar-angle range of $[17.2,152.6]$ degrees, which corresponds to $92 \%$ of the solid angle in the PEP-II center-off-mass frame, traverse at least 20 DCH layers. The DCH field wires (Fig. 3.10) are grounded, and the sense, guard, and clearing wires are operated at $1930 \mathrm{~V}, 340 \mathrm{~V}$, and $825 \mathrm{~V}$ (superlayer 1) or $883 \mathrm{~V}$ (superlayer 10), respectively.

The chamber is filled with an 80:20 mixture of helium and isobutane with an admixture of $3500 \mathrm{ppm}$ of water vapor and $70 \mathrm{ppm}$ of oxygen [48] in order to limit DCH aging due to the Malter effect $[50,49]$. Despite minor signs of aging, the DCH is expected to perform well as the PEP-II peak luminosity continues to increase and to survive through the end of the experiment. 


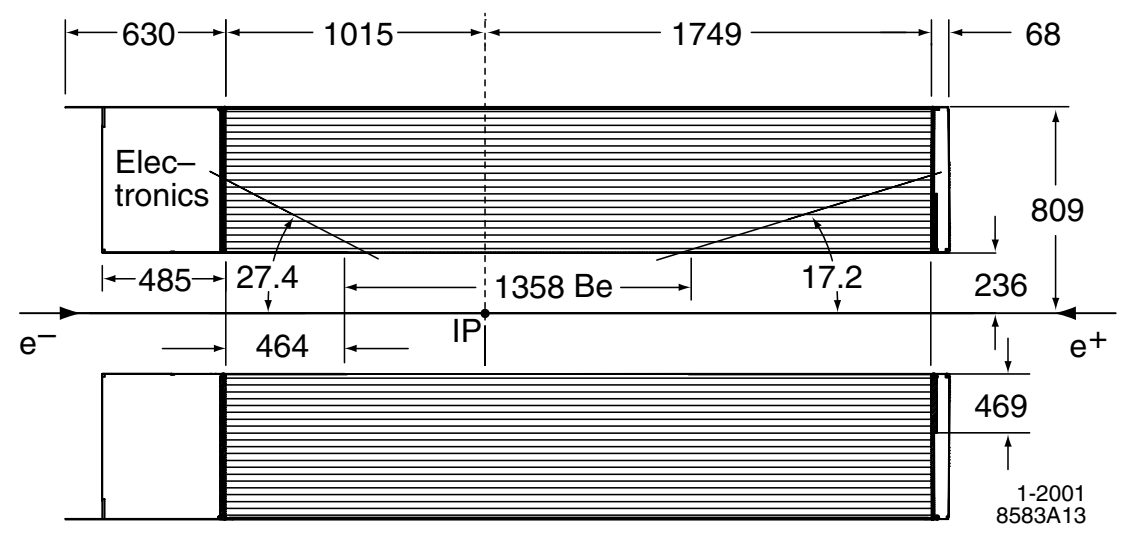

Figure 3.9: Longitudinal section of the $B A B A R$ Drift Chamber. Principal dimensions (in mm) are shown. The center of the chamber is offset by $370 \mathrm{~mm}$ from the PEP-II interaction point (IP), which optimizes the chamber's acceptance in the PEP-II center-of-mass frame.

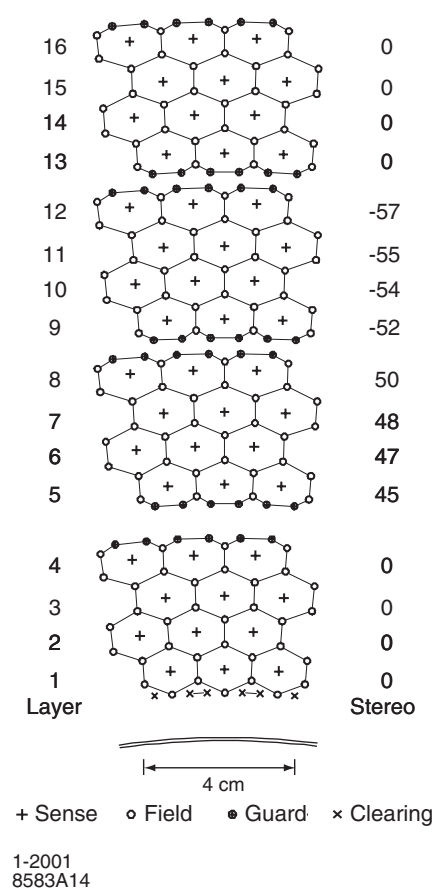

Figure 3.10: Schematic layout of DCH drift cells for the four innermost superlayers (there are four layers per superlayer). Lines connecting the field wires help in visualizing the drift-cell boundaries. The numbers in the "Stereo" column on the right indicate the stereo angles (in mrad) of the sense wires in each layer. Shown at the bottom of the drawing is the $1 \mathrm{~mm}$-thick beryllium inner wall of the drift chamber. 


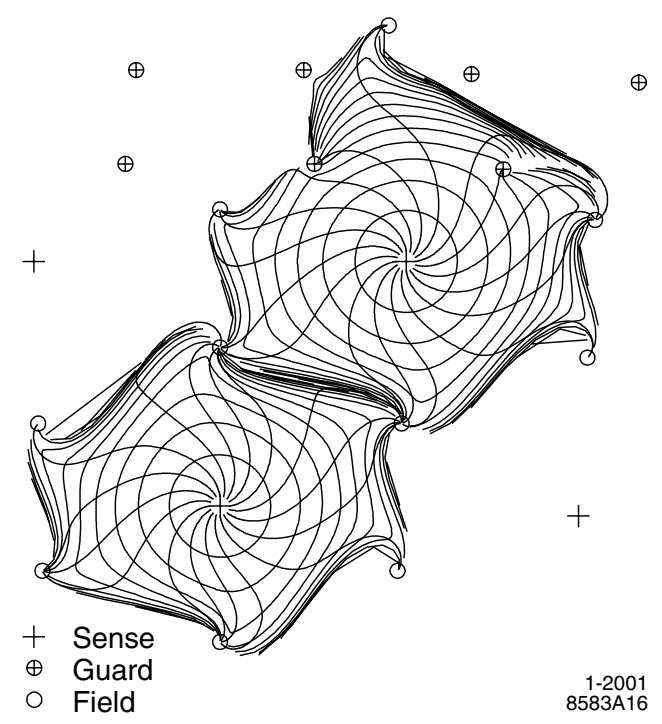

Figure 3.11: Illustration of DCH drift-cell isochrones (contours of equal ion drift times). The isochrones, spaced by $100 \mathrm{~ns}$ in this drawing, are circular near the sense wires but become irregular near the field wires.

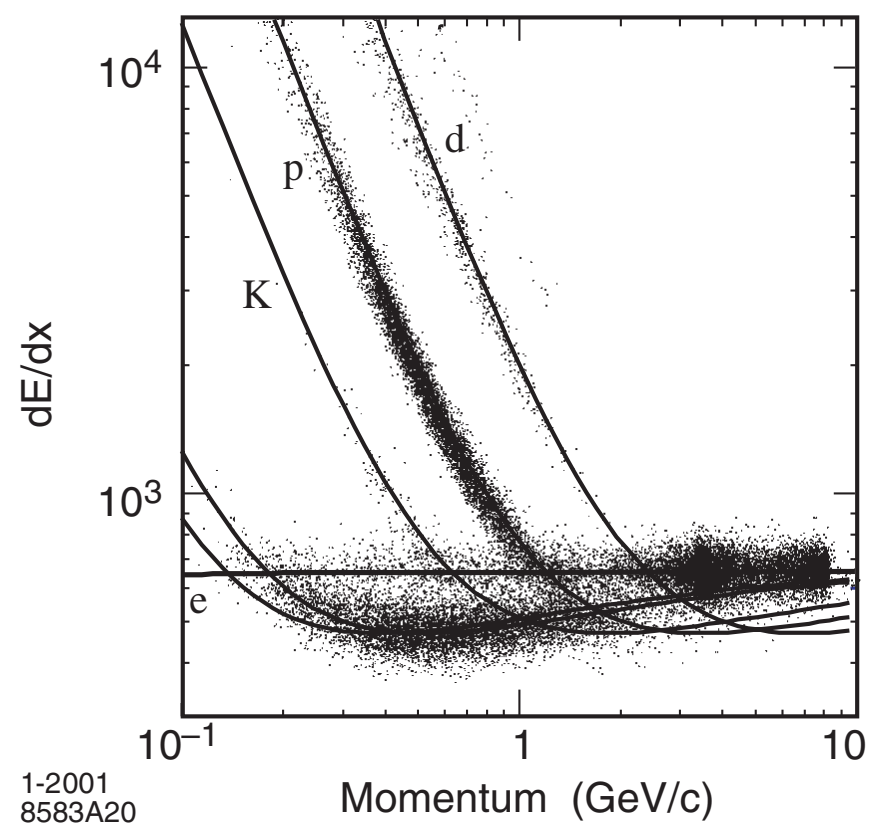

Figure 3.12: Measurement of ionization energy loss in the DCH as a function of track momenta. The curves show the Bethe-Bloch predictions for the charged particles of different types. The data include a large number of beam-background events, which results in the high rate of protons. 
Based on cosmic-ray data, the momentum resolution of the BABAR tracking system is well parametrised by

$$
\sigma_{p_{T}} / p_{T}=\left(0.13 p_{T}+0.45\right) \%
$$

where the transverse momentum $p_{T}$ is measured in $\mathrm{GeV} / c$. Since the tracking efficiency of the SVT alone for tracks that cross all of its five layers $\left(p_{T} \gtrsim 50-70 \mathrm{MeV} / c\right)$ is above $80 \%$, and the tracking efficiency of the DCH for tracks with $p_{T} \gtrsim 200 \mathrm{MeV} / c$ is approximately $98 \%$, the probability for a track to be reconstructed by the combination of the SVT and the DCH is very close to $100 \%$. With the DCH reconstruction-quality requirements of the GoodTracksLoose list applied (among them, a minimum of 12 DCH hits, see page 57), the reconstruction efficiency is approximately $97 \%$ for tracks with $p_{T} \gtrsim 1 \mathrm{GeV} / c$ and slightly lower for tracks with $p_{T}$ below $1 \mathrm{GeV} / c$ [88].

\subsection{Charged-particle identification}

The DIRC, BABAR's innovative ring-imaging Cherenkov detector [51, 52], is the dominant source of particle-idenification (PID) information for tracks with $p \gtrsim 700 \mathrm{MeV} / c$, i.e., above the $\sim 500 \mathrm{MeV} / c$ Cherenkov-radiation threshold for kaons. For lower-momentum tracks, PID is provided by the ionization energy loss in the SVT and the DCH (see Fig. 3.12). Electrons are identified with the use of the tracking system and the EMC.

The active detector medium of the DIRC is 144 bars of synthetic fused silica, each 


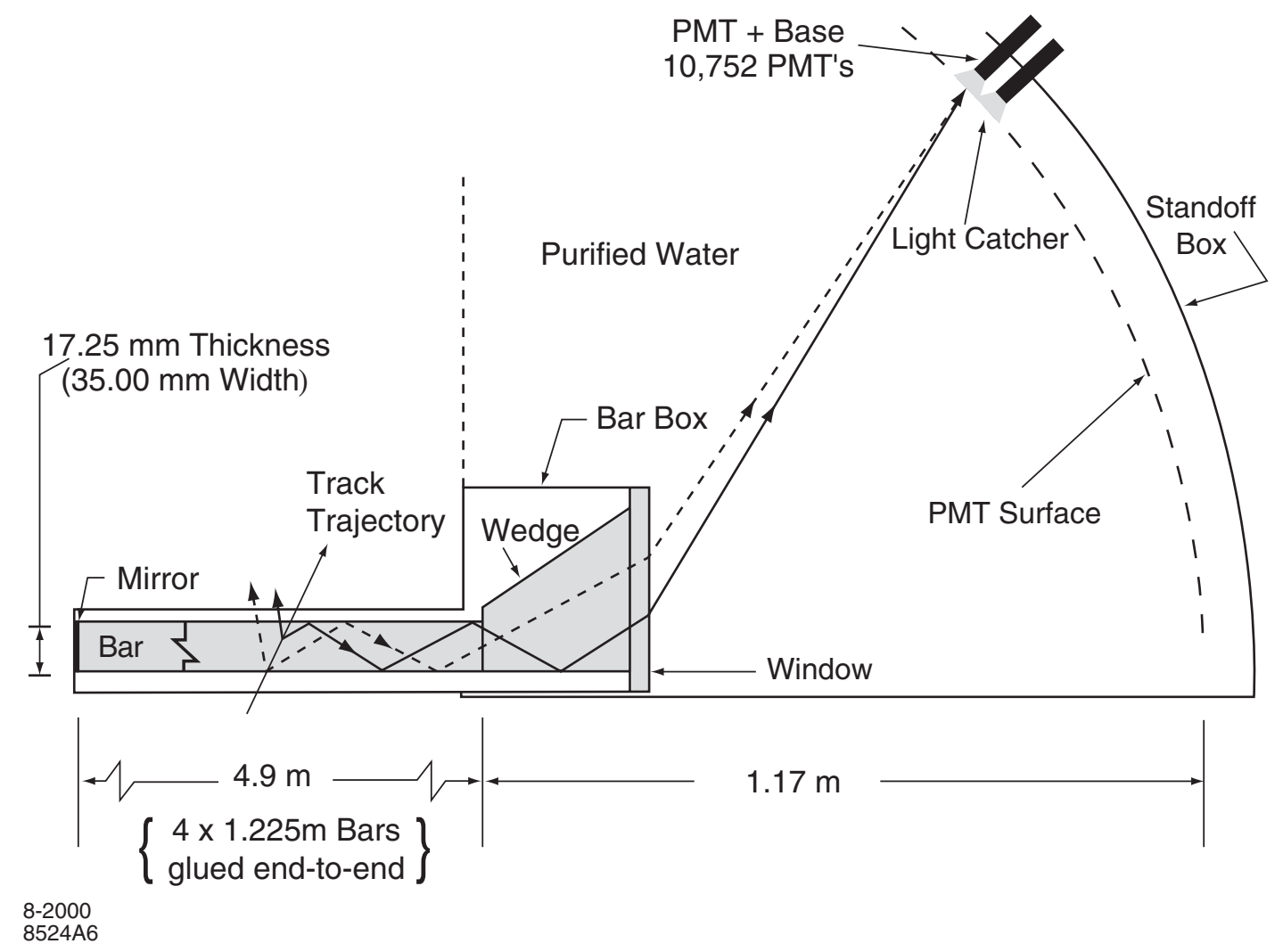

Figure 3.13: Illustration of the DIRC principle. Thanks to the phenomenon of total internal reflection, the cone of Cherenkov light gets partially trapped inside the fused-silica radiator bar and then imaged onto the spherical inner surface of the water-filled DIRC standoff box that is instrumented with 10752 photomultipler tubes. The bottom surface of the wedge is tilted by 6 mrad to achieve better focusing. 


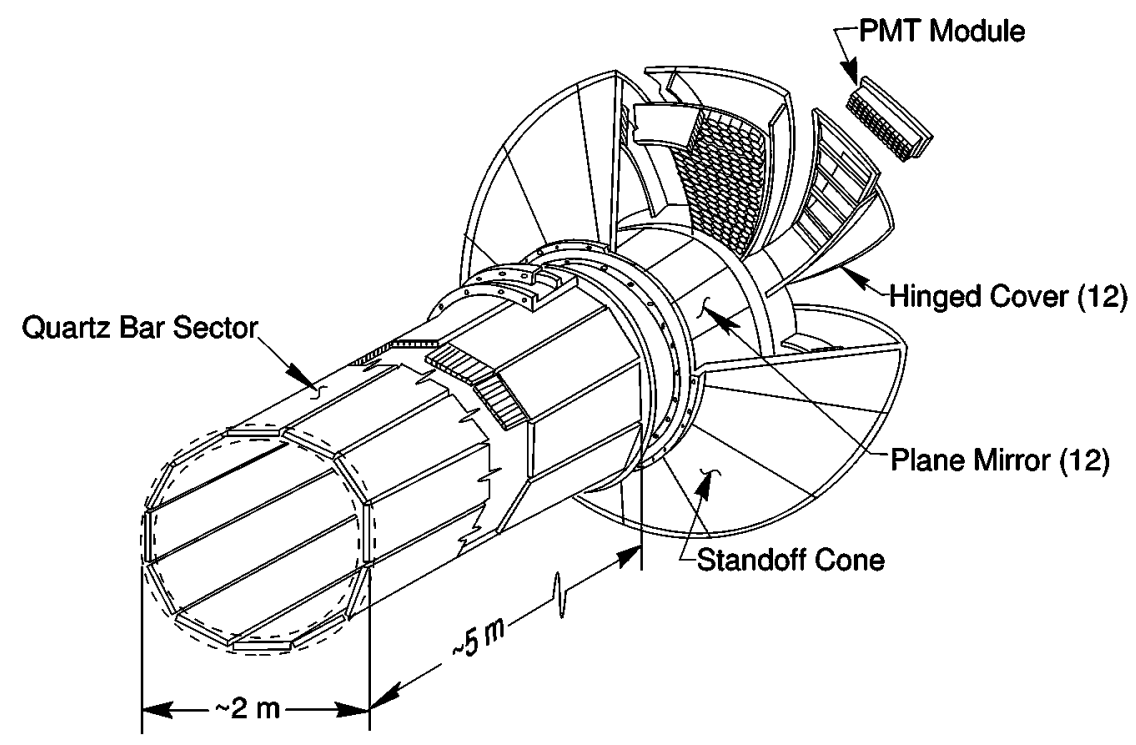

Figure 3.14: Schematic 3-D view of the DIRC.

$1.7 \mathrm{~cm}$ by $3.5 \mathrm{~cm}$ in cross section and $4.9 \mathrm{~m}$ in length. Since no technology exists that would allow manufacture of such a sufficiently finely and precisely polished 4.9-meterlong fused-silica bar, each of the 144 bars consists of four shorter bars glued together. The bars are placed into 12 bar boxes, which encircle the DCH (Figs. 3.13, 3.14).

Cherenkov light emitted by a charged particle moving faster than the speed of light $v_{\mathrm{Ch}}=c / n$ through a DIRC fised-silica bar $(n=1.473)$ is propagated inside the bar by total internal reflection to the rear end of the BABAR detector, then exits the bar into the standoff box (SOB) filled with six tons of purified water, where the Cherenkov angle (and thus, the velocity) of the particle is reconstructed by an array of 10752 photomultiplier tubes. The typical number of detected Cherenkov photons is $25-35$.

The DIRC covers the polar angle range of $[25.5,141.4]$ degrees, or $84 \%$ of polar angle cosine in the PEP-II center-of-mass frame; the azimuthal coverage is approximately 
94\%. Within its acceptance, the DIRC is $97 \%$ efficient for $3 \mathrm{GeV} / c$ tracks. The DIRC provides $\pi / K$ separation of better than $2.4 \sigma$ for all momenta kinematically accessible in $B$-meson decays (see Fig. 11.9 on page 121).

Since a leak of water from the SOB into a bar box through the seal of the barbox window or breakage of the window due to an earthquake could potentially lead to extensive damage to the hydrophobic CsI crystals inside the EMC and significant damage to other components of the BABAR detector, an automatic fault-tolerant waterleak detection system was designed that is capable of completely draining the SOB within a few minutes of detecting evidence of a water leak [53]. Liquid-water sensors include 20 custom-designed highly sensitive sensors that monitor each of the bar boxes and bar-box slots and two commercial ultrasonic water sensors that monitor each of the two drain lines that would collect water from the bar-box slots should a major leakage occur. Additionally, 12 commercial humidity sensors monitor the nitrogen-gas output lines from each of the bar boxes. So, there are four sensors associated with each of the bar boxes; the SOB is drained if any two of the four sensors detect the presence of water at the same time. While utility power may go out in case of an earthquake, power to the DIRC water-detection system and several other critical BABAR subsystems will continue to be provided for about a day by the BABAR uninterruptible power-supply (UPS) system; should the BABAR UPS become drained or damaged, battery backup allows an operator to drain the SOB manually without the need to enter the potentially more hazardous detector hall.

None of the DIRC detector components are sensitive to radiation damage. Some of 
the DIRC photomultiplier tubes are experiencing window damage due to the leaching of sodium and boron into water [51]; however, the extent of this damage is expected to remain acceptable over the lifetime of the experiment. Following an electronics upgrade that took place during the Fall 2002 shutdown, the DIRC data-acquisition system should be able to handle the event rates expected at PEP-II luminosities of up to $2 \times 10^{34} \mathrm{~cm}^{-2} \mathrm{~S}^{-1}$

\subsection{The Electromagnetic Calorimeter}

The BABAR Electromagnetic Calorimeter [45] is capable of measurement of the energy, position, and shape of electromagnetic showers in the energy range of $20 \mathrm{MeV}$ to $9 \mathrm{GeV}$. This enables reconstruction of the $\pi^{0} \rightarrow \gamma \gamma$ and $\eta \rightarrow \gamma \gamma$ decays, photons from electromagnetic and radiative processes, as well as identification of electrons.

The EMC (Fig. 3.15) consists of 6580 crystals made of CsI salt doped with $0.1 \%$ thallium, 16.0 to 17.5 radiation-lengths thick. The light yield of the CsI(Tl) medium is approximately $50 \times 10^{3}$ photons per $\mathrm{MeV}$; the emitted light, which peaks at the wavelength $\lambda_{\max }=565 \mathrm{~nm}$, is detected by a pair of photodiodes. The EMC acceptance in the PEP-II center-of-mass frame is $90 \%$.

Based on a fit to $\chi_{c 1} \rightarrow J / \psi \gamma$ and Bhabha-scattering data, the EMC energy resolution is

$$
\sigma_{E} / E=2.3 \% / \sqrt[4]{E(\mathrm{GeV})} \oplus 1.4 \%
$$




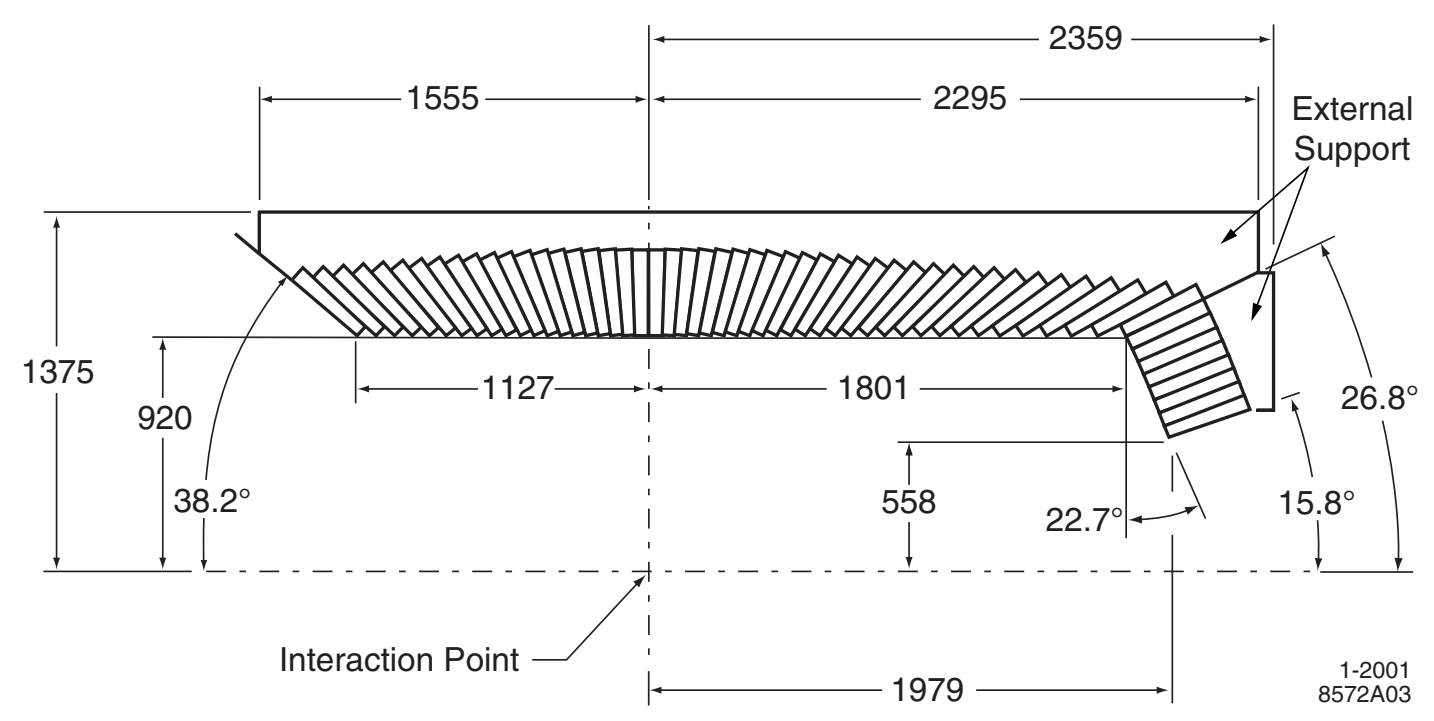

Figure 3.15: Schematic drawing of the longitudinal section of the EMC (only the top half is shown). All dimensions are given in $\mathrm{mm}$. The barrel part of the EMC contains 5760 crystals arranged in 48 rings with 120 crystals each; the front endcap holds 820 crystals arranged in eight rings.

The angular resolution is given approximately by $\sigma_{\theta}=\sigma_{\phi}=3.9 / \sqrt{E(\mathrm{GeV})} \mathrm{mrad}$.

Radiation damage to the $\mathrm{CsI}(\mathrm{Tl})$ crystals, which is the greatest in the forward endcap, causes a light-yield loss of $0.1 \%$ to $0.2 \%$ per $1 \mathrm{fb}^{-1}$ that is approximately linear with the integrated luminosity. It is, however, expected that the EMC energy and angular resolutions will not degrade significantly during the lifetime of the BABAR experiment [54].

\subsection{The Instrumented Flux Return}

The primary purpose of the BABAR Instrumented Flux Return [45] is to identify muons by taking advantage of their ability to penetrate through the steel of the solenoid magneticflux return. The IFR also provides directional information on neutrons and $K_{L}^{0}$ 's that 


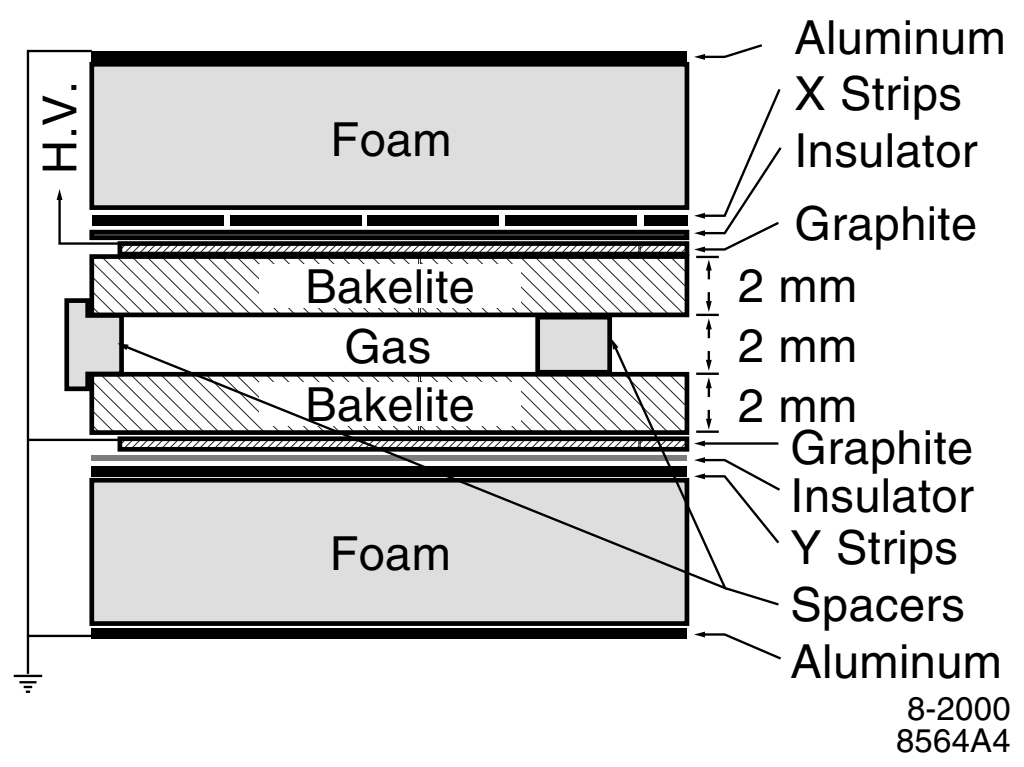

Figure 3.16: Cross section of an IFR planar single-gap resistive-plate chamber. The bakelite surfaces facing the gap are treated with linseed oil. RPCs operate by detecting streamers from ionizing particles via external capacitive readout strips.

interact within its volume. The analysis described in this dissertation does not use any information provided by the IFR.

The IFR consists of the barrel and two endcap sections (Fig. 3.5), each of which contains 19 layers of steel with 18 gaps that are filled with a total of 806 resistive-platechamber (RPC) modules (Fig. 3.16); the barrel has an extra, nineteenth, RPC layer. The total active area of RPCs in the IFR is approximately $2000 \mathrm{~m}^{2}$.

Chosen for their simplicity and cost-effectiveness, the original BABAR RPCs suffer from a significant, progressive loss of efficiency that has been traced to polymerization properties of linseed oil at elevated temperatures [55]. Chambers in the front endcap, which is the most important part of the IFR because of the PEP-II boost, were replaced with an updated version of RPCs in Summer 2002; plans for replacing barrel RPCs with 
limited-streammer tubes during the Summer 2004 and Summer 2005 shutdowns have recently been finalized [56].

\subsection{Trigger}

The role of a trigger system in a particle-physics experiment is provide efficient selection of events of interest with while rejecting most of the background events and keeping the resulting data rate under the maximum rate that the experiment's data-storage and dataprocessing systems can handle.

The design goal of the BABAR trigger system [45], which consists of the hardware Level 1 (L1) trigger and the software Level 3 (L3) trigger, is to have a total efficiency exceeding $99 \%$ for $B \bar{B}$ events and an efficiency above $95 \%$ for continuum $q \bar{q}$ events; requirements to the $e^{+} e^{-} \rightarrow \tau^{+} \tau^{-}$and $e^{+} e^{-} \rightarrow e^{+} e^{-} \gamma \gamma$ efficiencies are less stringent. A small fraction of $e^{+} e^{-} \rightarrow e^{+} e^{-}, e^{+} e^{-} \rightarrow \mu^{+} \mu^{-}, e^{+} e^{-} \rightarrow \gamma \gamma$, cosmic and beam background events are also passed through for use in detector calibration, diagnostic, and efficiency studies.

At the present peak luminosity of $6.6 \times 10^{33} \mathrm{~cm}^{-2} \mathrm{~S}^{-1}$, the combined $B \bar{B}+q \bar{q}+\tau^{+} \tau^{-}$ production rate is approximately $36 \mathrm{~Hz}$; additionally, approximately $8 \mathrm{~Hz}$ is due to $e^{+} e^{-} \rightarrow \mu^{+} \mu^{-}$events and approximately $350 \mathrm{~Hz}$ is due to $e^{+} e^{-} \rightarrow e^{+} e^{-}$events, where at least one of the two final-state particles falls within the EMC acceptance. The beaminduced background rate is typically around $40-60 \mathrm{kHz}$ for at least one DCH track with $p_{T}>120 \mathrm{MeV} / c$ or at least one EMC cluster with $E>100 \mathrm{MeV}$. 
The L1 trigger is configured to limit the normal-operation event rate to approximately $2.0 \mathrm{kHz}$ at the peak luminosity of $6.6 \times 10^{33} \mathrm{~cm}^{-2} \mathrm{~s}^{-1}$. It consists of two "production" triggers that are based on DCH (DCT) and EMC (EMT) information; an IFRbased trigger (IFT) is used for diagnostics. With the planned DCT upgrade [57], the L1 trigger is expected to accomodate the planned increase in the PEP-II luminosity.

The L3 trigger, implemented entirely in software, further limits the data rate to about $230 \mathrm{~Hz}$ at $L=6.6 \times 10^{33} \mathrm{~cm}^{-2} \mathrm{~s}^{-1}$ by applying track-finding and clustering algorithms to the events passed by the L1 trigger. Event of the types that are subject to rate reduction ("prescaling"), such as $e^{+} e^{-} \rightarrow \mu^{+} \mu^{-}, e^{+} e^{-} \rightarrow \gamma \gamma$, and $e^{+} e^{-} \rightarrow e^{+} e^{-}(\gamma)$, are identified as such by the L3 trigger. In responce to the increasing PEP-II luminosity, the L3-trigger software and computer hardware have recently been upgraded; further upgrades are being planned [58].

\subsection{The data set}

This analysis is based on an integrated luminosity of about $82 \mathrm{fb}^{-1}$, corresponding to approximately 89 million $B \bar{B}$ pairs, collected by $B A B A R$ in $1999-2002$. An additional sample with an integrated luminosity of $9.6 \mathrm{fb}^{-1}$ was collected $40 \mathrm{MeV}$ below the $\Upsilon(4 S)$ resonance (off-resonance) and used for the study of continuum backgrounds.

All data used in the analysis were reconstructed with release 10-series of the BABAR software; release 10.4.0-physics-1a (a.k.a. analysis-12a) was used in all ntuple production. We used the Micro Data Store in the ROOT-based KANGA format [59] for most of 
the 1999, 2000 and 2001 data, and switched to using data stored in the Objectivity database for data collected or reprocessed in 2002. Results of running on data stored in the KANGA and Objectivity formats were thoroughly compared and found to be identical. Particular care has been exercised to exclude or substitute 1999-2001 runs that were declared unsuitable for physics analysis or reprocessed after the ntuple production for pre-2002 data was completed. 


\section{Chapter 4}

\section{Terminology}

We have chosen to devote a separate section to definitions of variables, candidate lists and some specialized jargon used throughout the note. Readers who are well familiar with the methodology commonly used in BABAR and specifics of exclusive analysis of $B$-meson decays into fully reconstructable final states are encouraged skip to Section 6 (page 63). Definitions of some more general terms are given in Appendix A.

\subsection{Variables}

- $m_{\mathrm{ES}}$ : "beam-energy-substituted mass", computed in the laboratory frame and independent of mass hypotheses assigned to $B$ candidate daughters:

$$
m_{\mathrm{ES}}=\sqrt{\frac{\left(\frac{s}{2}+\vec{p}_{\Upsilon(4 S)} \cdot \vec{p}_{B}\right)^{2}}{E_{\Upsilon(4 S)}^{2}}-\vec{p}_{B}^{2}},
$$

where $q_{\Upsilon(4 S)}$ and $q_{B}$ are four-momenta of the $\Upsilon(4 S)$ and the $B$ candidate, $s \equiv$ 
$\left(q_{\Upsilon(4 S)}\right)^{2}$ is the square of the center-of-mass energy, $\vec{p}_{\Upsilon(4 S)}$ and $\vec{p}_{B}$ are threemomenta of the $\Upsilon(4 S)$ and the $B$ in the laboratory frame, and $E_{\Upsilon(4 S)} \equiv q_{\Upsilon(4 S)}^{0}$ is the energy of the $\Upsilon(4 S)$ in the laboratory frame.

- $\boldsymbol{\Delta} \boldsymbol{E}$ : "delta E", a Lorentz-invariant quantity defined as

$$
\Delta E=\frac{2 q_{\Upsilon(4 S)} q_{B}-s}{2 \sqrt{s}}
$$

$-\Delta E$ and $\boldsymbol{m}_{\mathrm{ES}}$ is our choice of the pair of weakly-correlated variables used to characterize kinematics of $B$ meson candidates. You may refer to [78] for more information and a discussion of alternatives. Although $m_{\mathrm{EC}}$ is the constrainedmass variable that correlates the least with $\Delta E$ for signal events, use of $m_{\mathrm{EC}}$ induces such a correlation on continuum background events (e.g. see Fig. 4.1 and Fig. 4.2). Since such a correlation could introduce a bias into our results, this is our prime reason for not using $m_{\mathrm{EC}}$, the other reason being the dependence of $m_{\mathrm{EC}}$ on the $B$ daughter mass assignment.

- $\left|\cos \theta_{T}\right|$ : The absolute value of the cosine of the angle between the thrust axes (see Appendix A) of the $B$ candidate daughters and the "Rest-of-the-Event" $(\mathrm{ROE})^{1}$ is a powerful tool used by many $B A B A R$ analyses to discriminate against continuum background. Since $B$ 's are non-relativistic in the $\Upsilon(4 S)$ rest frame $(\beta \approx 0.06)$, the $\left|\cos \theta_{T}\right|$ distribution for true $B$ candidates is very well described by a first-degree polynomial, the slope being mostly due to mode-dependent acceptance effects.

\footnotetext{
${ }^{1}$ See page 58 for a detailed definition of "Rest-of-the-Event".
} 

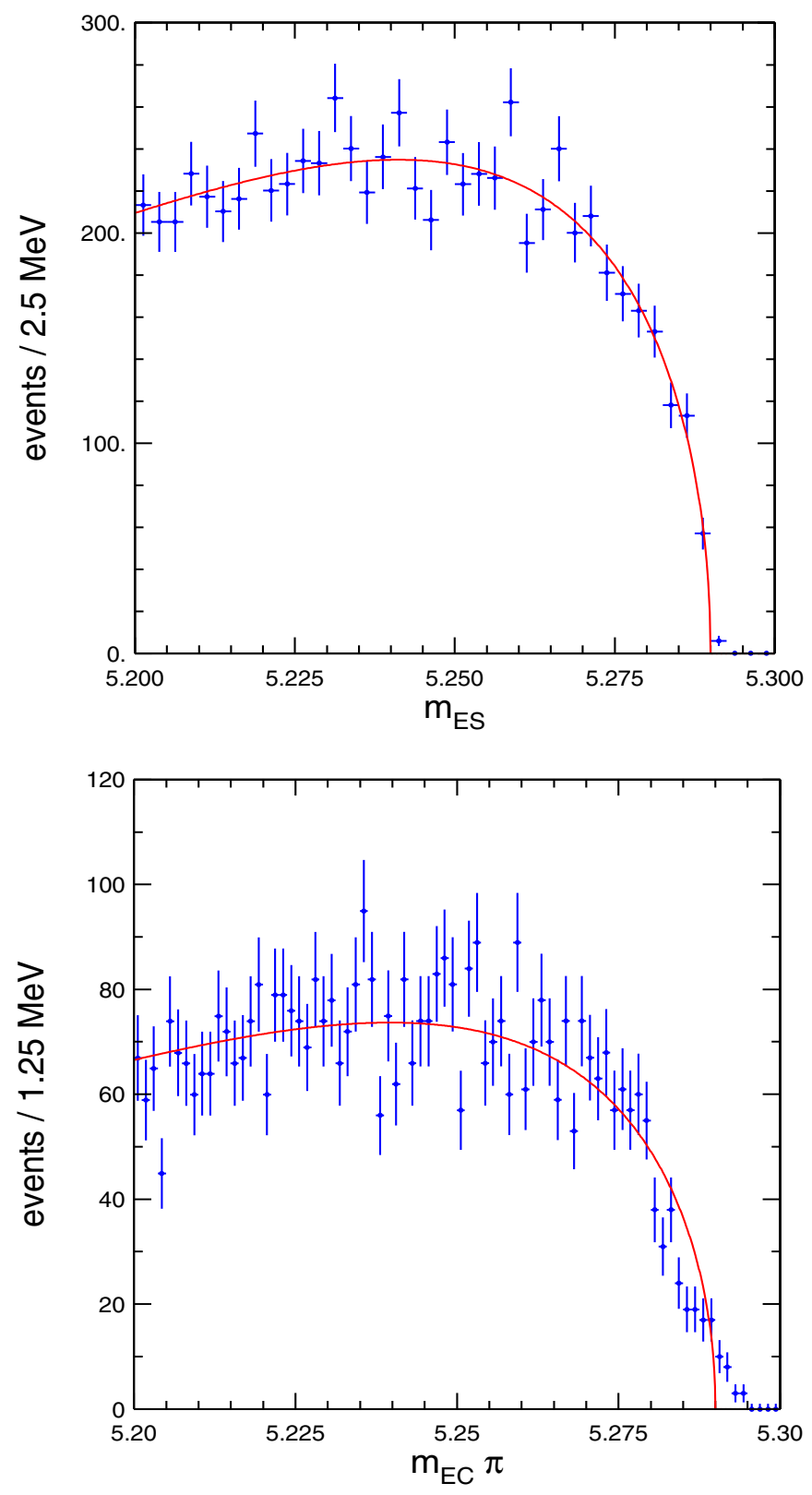

Figure 4.1: (top) $m_{\mathrm{ES}}$ and (bottom) $m_{\mathrm{EC}}$ distributions for $B^{ \pm} \rightarrow \phi h^{ \pm}$candidates in the onresonance sideband $\left(5.20<\left(m_{\mathrm{ES}}, m_{\mathrm{EC}}\right)<5.30 \mathrm{GeV} ; 0.1<\left|\Delta E_{\pi, K}\right|<0.2 \mathrm{GeV}\right)$ in 19992001 data fitted with the phenomenological ARGUS function (see page 144). Note that the right edge of the $m_{\mathrm{EC}}$ distribution is poorly described by the ARGUS function, which is a result of the dependence of the $m_{\mathrm{EC}}$ end point on the value of $\Delta E$ (see Fig. 4.2). 


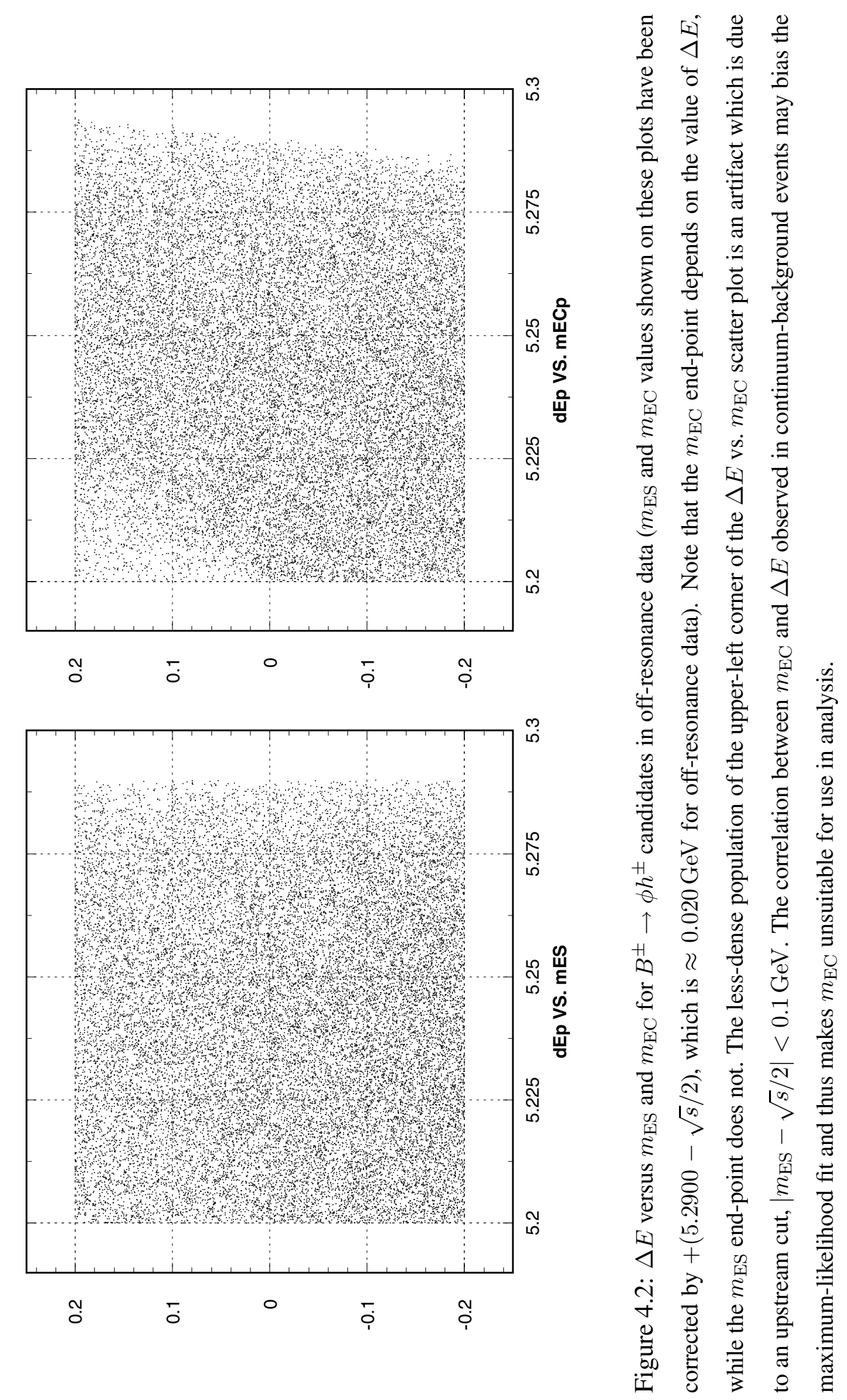



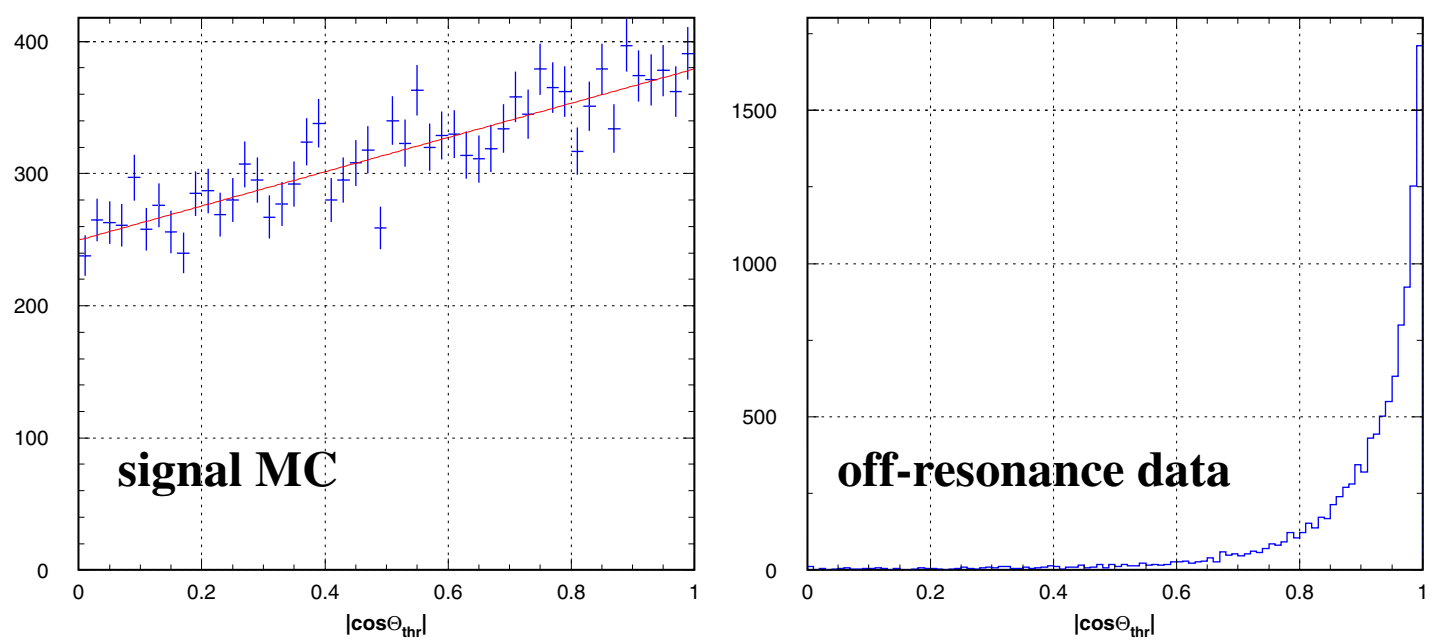

Figure 4.3: $\left|\cos \theta_{T}\right|$ distributions for (left) $B^{ \pm} \rightarrow \phi K^{ \pm}$signal Monte Carlo and (right) $B^{ \pm} \rightarrow$ $\phi h^{ \pm}$candidates in off-resonance data (continuum background).

On the other hand, the $\left|\cos \theta_{T}\right|$ distribution for $B$ candidates found in the $u d s c \tau$ continuum is sharply peaked at 1 (Fig. 4.3, Fig. 4.4). Because of this, the dependence of the $\left|\cos \theta_{T}\right|$ distribution on the type of continuum background (Fig. 4.4), and, most importantly, due to the fact that $\left|\cos \theta_{T}\right|$ is strongly correlated with another event shape variable, $\mathcal{F}$ (described below), it is imperative to cut on $\left|\cos \theta_{T}\right|$ rather than attempt using it as an input variable in the maximum-likelihood fit. We use the cut $\left|\cos \theta_{T}\right|<0.9$ throughout our analysis, which is justified by the relatively low amounts of combinatorial (random-track) background in the decay $\operatorname{modes} B^{ \pm} \rightarrow \phi h^{ \pm}$and $B^{0} \rightarrow \phi K_{S}^{0}$.

- Fisher discriminant $\mathcal{F}$ : In 1936, Ronald Fisher, then professor of Eugenics at the University of London, proposed [60] a way to discriminate among two or more populations or species for which several "characters", or variables, $x_{i}$ can 


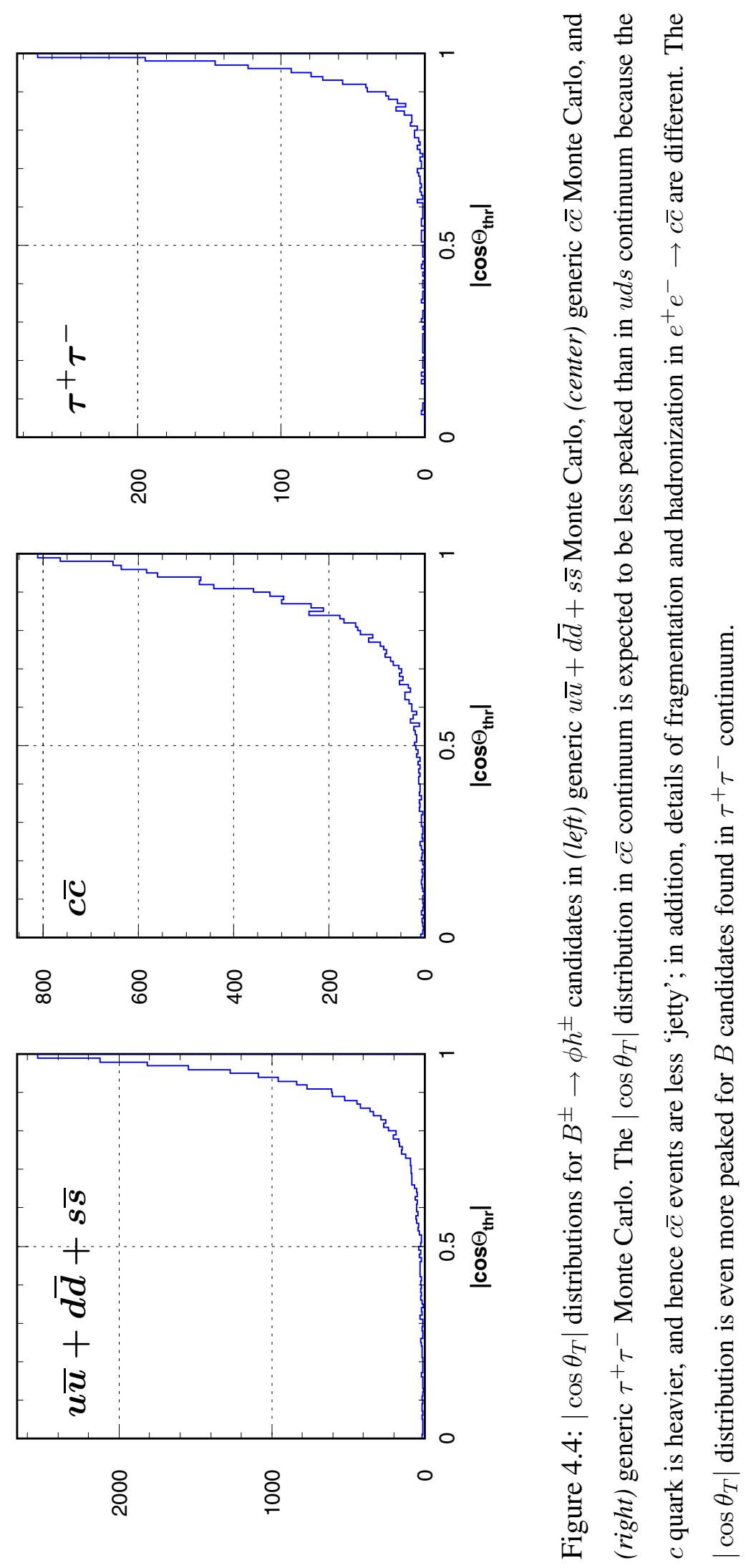


be measured by composing a linear function of these characters

$$
\mathcal{F}=\sum \lambda_{i} x_{i}
$$

where the coefficients $\lambda_{i}$ are chosen in a way that maximizes significance of the difference between $\mathcal{F}$ distributions for the two or more populations. In general, a neural network would provide a better separation than a Fisher discriminant by exploiting the possible non-linear relations among the input variables; in practice, as shown by research conducted within both CLEO and BABAR collaborations, the benefit of using a neural network is usually minimal.

In the preliminary version of this analysis, which was based on $56.3 \mathrm{fb}^{-1}$ of 1999-2001 data and presented at the DPF 2002 conference in May 2002 [61], we employed a set of eleven variables originally proposed by Frank Würthwein, then a graduate student in the CLEO Collaboration, and known within BABAR as the "CLEO Fisher": the first two are the absolute values of cosines of the angles between the $z$ axis of the detector (or, more appropriately in the case of $B A B A R$, the beam axis) and (1) the momentum of the $B$ candidate $\left(\left|\cos \theta_{p_{B}}\right|\right.$, which is distributed as $\sin ^{2} \theta$ for $\Upsilon(4 S) \rightarrow B \bar{B}$ and has a flat distribution for continuum background) and (2) the thrust axis of $B$ candidate daughters $\left(\left|\cos \theta_{B_{\mathrm{thr}}}\right|\right.$, which, neglecting acceptance effects near 1 , is uniformly distributed for $B$ decays and is peaked toward 1 for continuum background, see Fig. 4.5 and Fig. 4.6). The other nine variables, known as the "virtual calorimeter", are "momentum flows" 

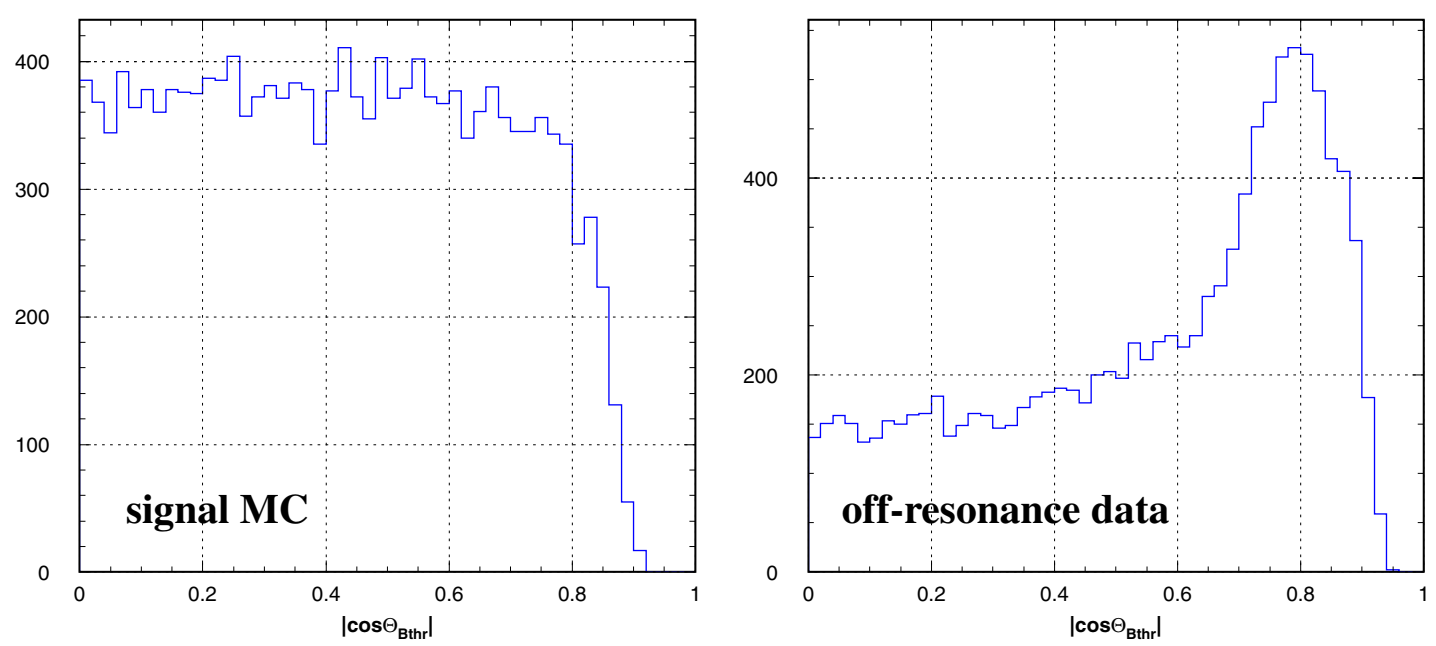

Figure 4.5: $\left|\cos \theta_{B_{\mathrm{thr}}}\right|$ distributions for (left) $B^{ \pm} \rightarrow \phi K^{ \pm}$signal Monte Carlo and (right) $B^{ \pm} \rightarrow \phi h^{ \pm}$candidates in off-resonance data (continuum background).

(scalar sums of momenta) of ROE particles into the nine "CLEO cones", which are centered around the $B$ candidate's thrust axis and span 10 degrees each in both directions along the trust axis: $0^{\circ}$ to $10^{\circ}$ and $170^{\circ}$ to $180^{\circ}$ for the first cone, $10^{\circ}$ to $20^{\circ}$ and $160^{\circ}$ to $170^{\circ}$ for the second, etc. All calculations are done in the center-of-mass frame of the $\Upsilon(4 S)$. The set of coefficients $\lambda_{i}$ that we and most other analyses in the Charmless Analysis Working Group have been using was determined by Jean Roy in mid-2000 with a sample of $5000 \mathrm{~B}^{+} \rightarrow \omega \pi^{+}$Monte Carlo events and $0.14 \mathrm{fb}^{-1}$ of off-resonance data with the $\left|\cos \theta_{T}\right|<0.9$ cut applied. It has been shown that the same set of coefficients can be used in all charmless analyses with a minimal loss of the discriminating power of $\mathcal{F}$.

In the analysis presented in this dissertation, we use a "New Fisher", which replaces the nine terms of the CLEO "virtual calorimeter" with two quantities $P_{0}$ 


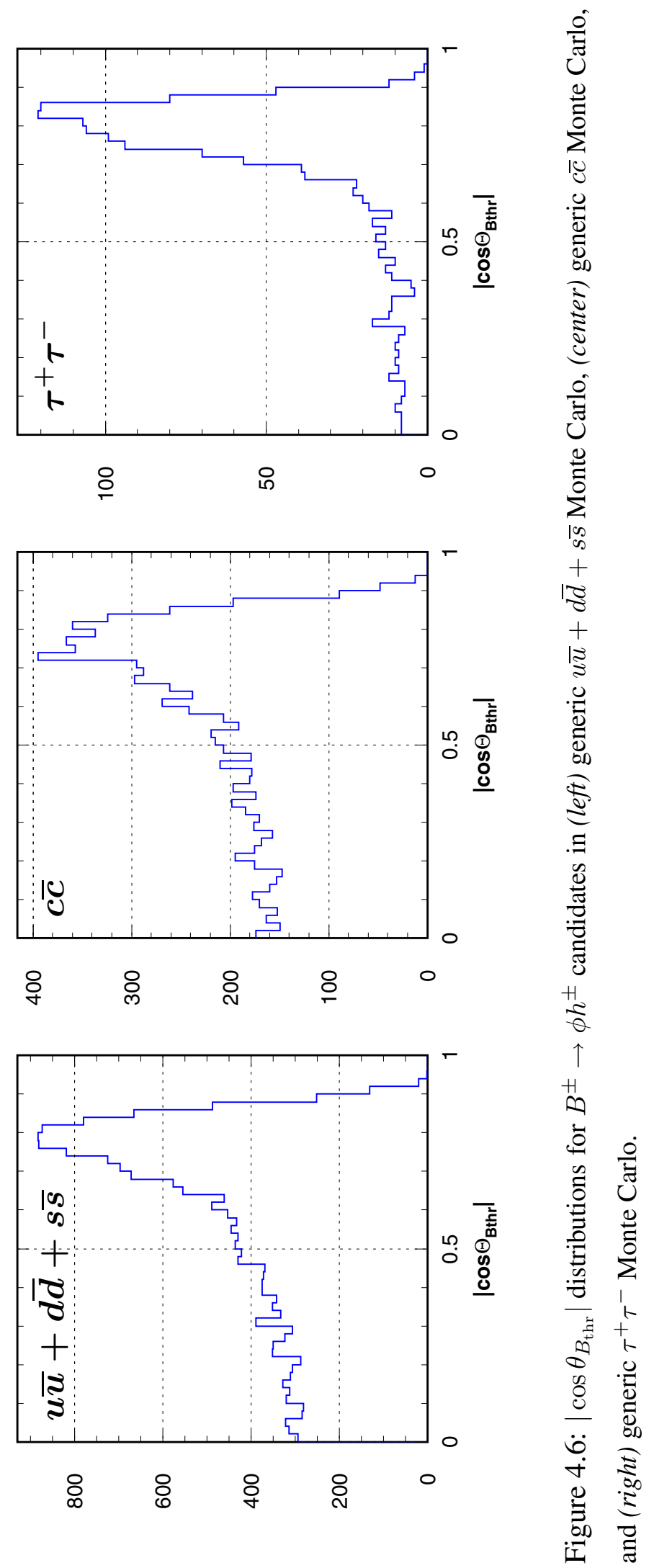


and $P_{2}$ defined as

$$
P_{n}=\sum_{i \in \mathrm{ROE}} p_{i} \times \cos ^{n}\left(\theta_{i}\right)
$$

where the sum is over all charged and neutral candidates in the Rest-of-the-Event, and $\theta_{i}$ is the angle between the momentum of the particle $i$ and the thrust axis of the $B$ candidate, both computed in the $\Upsilon(4 S)$ rest frame. Alternatively, one may use Legendre moments $\mathcal{L}_{n}$ of the ROE,

$$
\mathcal{L}_{n}=\sum_{i \in \mathrm{ROE}} p_{i} \times L_{n}\left(\theta_{i}\right)
$$

which are linear combinations of $P_{0} \ldots P_{n}$. The set of coefficients for the New Fisher used in this analysis had been determined by Frederic Blanc at the University of Colorado in a study of a variety of quasi-two-body charmless $B$ decay modes [102]. We studied the background suppression powers of the "CLEO" and the "New" Fisher discriminants in data on the high-statistics mode $B^{+} \rightarrow$ $\pi^{+} \bar{D}^{0}\left(\bar{D}^{0} \rightarrow K^{+} \pi^{-}\right)$, and came to a conclusion that the separation power of the New Fisher is indeed slightly better than that of the CLEO Fisher (see Fig. 4.7).

- Cherenkov angle pulls: defined for charged track candidates passing through the DIRC as the difference between the measured value and the values expected for various PID hypotheses, divided by the measurement error, of the opening angle of the Cherenkov ring emitted by the charged candidate. For a pure PID sample, 


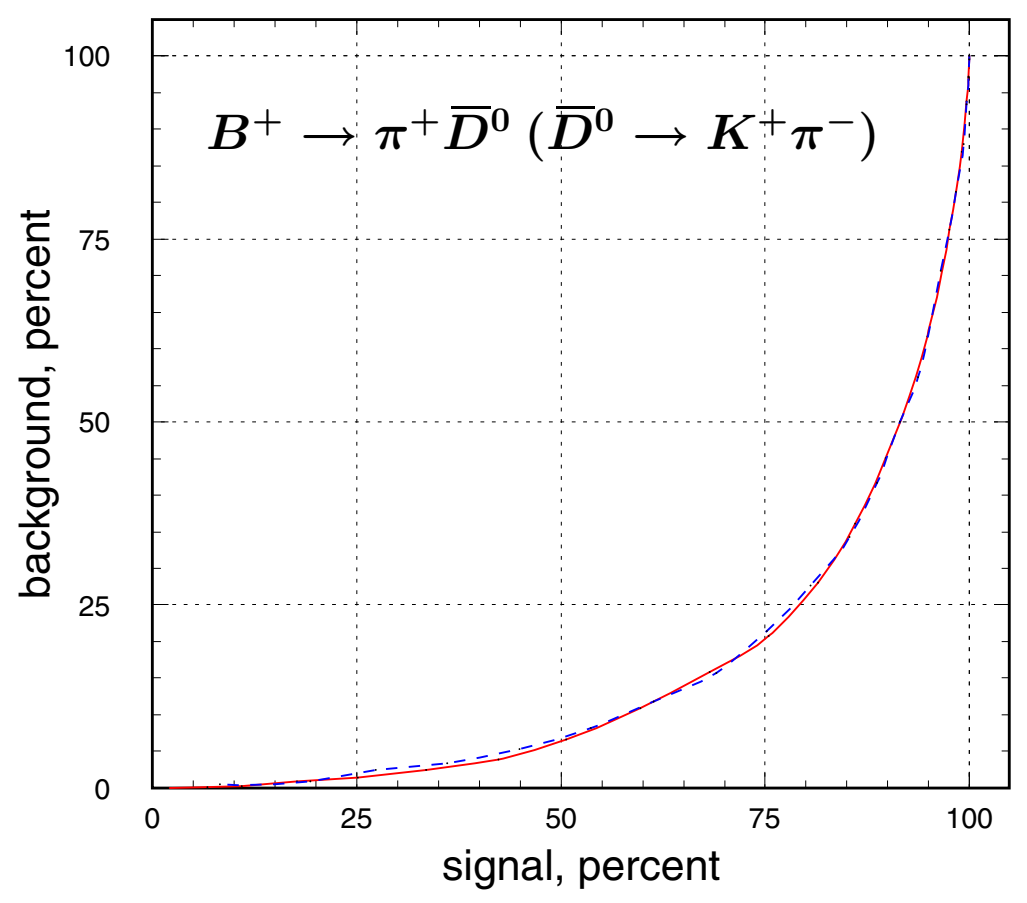

Figure 4.7: Signal-background separation curves for the CLEO Fisher discriminant (dashed line) and the Legendre-polynomial-based "New" ("BABAR") Fisher discriminant (solid line) in $B^{+} \rightarrow \pi^{+} \bar{D}^{0}\left(\bar{D}^{0} \rightarrow K^{+} \pi^{-}\right)$data.

the PDF of the corresponding pull is a unit Gaussian. We use latest (Summer 2002) version of the code developed by Carlo Dallapiccola for charmless twobody analyses to correctly compute Cherenkov angle pulls based on quantities stored in the Micro Data Store $[83,96]$.

- $\cos \theta_{\text {hel }}(\mathcal{H})$ : cosine of what is colloquially referred to as the "helicity angle". We define helicity angle of a $\phi$ resonance candidate in the decay $\phi \rightarrow K^{+} K^{-}$as the angle between the direction of the positively-charged kaon and the direction of momentum of the $\phi$ 's parent (i.e., the $B$ candidate) in the $\phi$ 's rest frame. Note that this definition differs only in sign (which in our case is irrelevant) from a 
more conventional definition that talks about the initial direction of the $\phi$ coming from the rest frame of the $B$. Since $B \rightarrow \phi K$ is a decay of pseudoscalar into a pseudoscalar and a vector $\left(0^{-} \rightarrow 0^{-}+1^{-}\right)$, the $\phi$ resonance is fully polarized and $\cos \theta_{\text {hel }}$ in signal events is distributed as $\left(\cos \theta_{\text {hel }}\right)^{2} ; \phi$ candidates in the continuum background are only partially polarized.

\subsection{Lists}

Analyses in $B A B A R$ rely on a variety of standard per-event lists of reconstructed particle candidates. Lists relevant to this analysis are described below.

- ChargedTracks: Simply a list of all reconstructed charged tracks in the event, with the pion mass hypothesis assigned.

- GoodTracksVeryLoose: ChargedTracks with $p<10 \mathrm{GeV}$ and a distance of closest approach (DOCA) to the beamspot of less than $1.5 \mathrm{~cm}$ in the $X Y$ plane and less than $10 \mathrm{~cm}$ in $Z$.

- GoodTracksLoose: GoodTracksVeryLoose with a minimum of 12 drift chamber hits and a minimum transverse momentum of $0.1 \mathrm{GeV}$.

- KsDefault: formed of pairs of oppositely-charged ChargedTracks that have an invariant mass within $0.025 \mathrm{GeV}$ of the reference value of $m_{K_{S}^{0}}=0.497672 \pm$ $0.000031 \mathrm{GeV}$. 
- CalorNeutral: Candidates that are EMC bumps not matched with any charged track, mostly photons. Although the decay modes we are studying have only charged tracks in the final state, CalorNeutral objects do have relevance to our analysis because we use them, along with the remaining charged tracks, to study the shape of the "Rest-of-the-Event", which helps us discriminate against continuum background.

\subsection{Other terminology}

- Bachelor track or particle: a particle that is a direct product of $B$ meson decay rather than a product of decay of one of its daughters. For example, in $B^{ \pm} \rightarrow$ $\phi \pi^{ \pm}, \phi \rightarrow K^{+} K^{-}$, the pion would be a bachelor track. In $B A B A R$, the bachelor tracks' momentum spectrum in the laboratory frame spans from $\sim 1.4$ to $\sim 4.5$ $\mathrm{GeV}$, which means that $\pi^{ \pm} / K^{ \pm}$PID separation for bachelor tracks is dominated by Cherenkov angle determination in the DIRC.

- ROE: Rest-Of-the-Event, all candidates in the event that are not part of the $B$ candidate decay tree. Helps to discriminate against continuum background. In early days of $B A B A R$ some analyses were limiting themselved to using only charged candidates in the ROE. We include in ROE all unused GoodTracksVeryLoose tracks and CalorNeutral neutrals (but not IFR $K_{L}^{0}$ candidates). 


\section{Chapter 5}

\section{Maximum-Likelihood Fit}

We use an unbinned extended maximum-likelihood (ML) fit to extract signal yields and charge asymmetries simultaneously. The extended likelihood for a sample of $N$ events is

$$
\mathcal{L}=\exp \left(-\sum_{i, k} n_{i k}\right) \prod_{j=1}^{N}\left(\sum_{i, k} n_{i k} \mathcal{P}_{i k}\left(\vec{x}_{j} ; \vec{\alpha}\right)\right)
$$

where $\mathcal{P}_{i k}\left(\vec{x}_{j} ; \vec{\alpha}\right)$ is the probability density function (PDF) for measured variables $\vec{x}_{j}$ of an event $j$ in category $i$ and flavor state $k$, and $n_{i k}$ are the yields extracted from the fit. The fixed parameters $\vec{\alpha}$ describe the expected distributions of measured variables in each category and flavor state. The PDFs are non-zero only for the correct final state flavor $(k=1$ for $\bar{B} \rightarrow \bar{f}$ and $k=2$ for $B \rightarrow f$ ). In the simplest case, there are two categories, signal and background $(i=1,2)$. The decays with a charged primary daughter $B^{ \pm} \rightarrow \phi h^{ \pm}(h=\pi$ or $K)$ are fitted simultaneously with two signal $(i=1$ for $B^{ \pm} \rightarrow \phi K^{ \pm}$and $i=2$ for $\left.B^{ \pm} \rightarrow \phi \pi^{ \pm}\right)$and two corresponding background $(i=3,4)$ 
categories.

We define the event yields $n_{i k}$ in each category in terms of the asymmetry $\mathcal{A}_{i}$ and the total event yield $n_{i}: n_{i 1}=n_{i} \times\left(1+\mathcal{A}_{i}\right) / 2$ and $n_{i 2}=n_{i} \times\left(1-\mathcal{A}_{i}\right) / 2$. Note that the sign of the charge asymmetry is defined with respect to the flavor of the $b$ quark, i.e.,

$$
\mathcal{A}_{C P}=\frac{N_{b}-N_{\bar{b}}}{N_{b}+N_{\bar{b}}}=\frac{N_{B^{-}}-N_{B^{+}}}{N_{B^{-}}+N_{B^{+}}} .
$$

The event yields $n_{i}$ and asymmetries $\mathcal{A}_{i}$ in each category are obtained by minimizing the quantity $-\ln \mathcal{L}$. Statistical errors correspond to unit changes in the quantity $\chi^{2}=$ $-2 \ln \left(\mathcal{L} / \mathcal{L}_{\text {max }}\right)$. The significance of a signal is measured by the square root of the change in $\chi^{2}$ when the number of signal events is constrained to zero in the likelihood fit; it describes the probability for the background to fluctuate to the observed event yield.

The probability $\mathcal{P}_{i}\left(\vec{x}_{j} ; \vec{\alpha}\right)$ for a given event $j$ is the product of independent PDFs in each of the fit input variables $\vec{x}_{j}$. These variables are $\Delta E, m_{\mathrm{ES}}, m_{K K}$, the Fisher discriminant $\mathcal{F}$, and the cosine of the $\phi$ helicity angle (defined as the angle between the $K^{+}$ and $B$ momenta in the $\phi$ rest frame) $\cos \theta_{\text {hel }}$. In addition, in the simultaneous fit for the modes $B^{ \pm} \rightarrow \phi K^{ \pm}$and $B^{ \pm} \rightarrow \phi \pi^{ \pm}$we include normalized residuals derived from the difference between the measured and expected DIRC Cherenkov angles for the charged primary daughter. Additional separation between the two final states is provided by $\Delta E$. The $\Delta E$ separation depends on the momentum of the charged primary daughter in the laboratory frame and is about $45 \mathrm{MeV}$ on average, varying from about $30 \mathrm{MeV}$ 
for the highest-momentum to about $80 \mathrm{MeV}$ for the lowest-momentum primary daughters available in our final states. If a given event has multiple combinations satisfying the selection requirements (which occurs in fewer than $0.2 \%$ of the events), the "best" combination is selected using a $\chi^{2}$ quantity computed using all input variables with the exception, in the $B^{ \pm} \rightarrow \phi h^{ \pm}$case, of the normalized Cherenkov-angle residuals and $\Delta E$, which are used for $\phi \pi^{ \pm} / \phi K^{ \pm}$separation.

The fixed parameters $\vec{\alpha}$ defining the PDFs are extracted for signal from Monte Carlo simulation and for background distributions from the on-resonance sidebands in $m_{\mathrm{ES}}$ and $\Delta E$ (Fig. 8.1). The MC resolutions and means are adjusted, when necessary, by comparing data and simulation in abundant calibration channels with kinematics and topologies similar to signal, $B^{+} \rightarrow \pi^{+} \bar{D}^{0}\left(\bar{D}^{0} \rightarrow K^{+} \pi^{-}\right)$and $B^{0} \rightarrow \pi^{+} D^{-}\left(D^{-} \rightarrow\right.$ $\left.K^{0} \pi^{-}\right)$. The PDFs for the Cherenkov-angle residuals are determined from samples of $D^{0} \rightarrow K^{-} \pi^{+}$originating from $D^{*}$ decays.

We employ a double Gaussian to parametrize the signal $\Delta E$ and $m_{\mathrm{ES}}$ PDFs. For the background, a first-degree polynomial is used for $\Delta E$ and an empirical phase-space function [64] is used for $m_{\mathrm{ES}}$. The Fisher discriminant distributions both in signal and in background are parametrized by a Gaussian with different widths above and below the mean. The $\phi$-resonance shape in signal and the real- $\phi$ component of the continuum background are parametrized by the relativistic spin-1 Breit-Wigner function [62] with the Blatt-Weisskopf damping factor correction [63] convoluted with a Gaussian resolution function $\left(\sigma=1.0 \mathrm{MeV} / c^{2}\right)$; the combinatorial component of the $m_{K K}$ distribution in the continuum background is parametrized with a second-degree polynomial 
(Fig. 8.8b, 8.8c). Since $B \rightarrow \phi K$ and $B \rightarrow \phi \pi$ are decays of a pseudoscalar particle into a vector and a pseudoscalar, the helicity-angle distribution for the signal is $\cos ^{2} \theta_{H}$; the background shape is again separated into contributions from combinatorial sources and from real $\phi$ mesons, both of which are parametrized by second-degree polynomials with no linear terms. The Cherenkov-angle-residual PDFs are unit Gaussians for both the pion and kaon distributions.

For all modes, we test the fitting procedure with background samples generated according to the PDFs and signal from Monte Carlo simulation, with numbers of signal and background events close to the expected. Signal yields are found to be unbiased. Correlations among the input variables in data are less than 5\%. 


\section{Chapter 6}

\section{Event Selection and Preliminary Cuts}

\subsection{Skims}

The first round of event selection for most analyses in BABAR is the production of event skims. Whenever reconstruction of charged tracks and neutrals in an event from raw detector data is run, either in the process of "Online Prompt Reconstruction" (OPR) that takes place within a few hours after the data is taken, or during the approximately once-a-year offline data reprocessing, the event undergoes a series of relatively simple test that allow indentification ("physics tagging") of the event as a possible candidate for one or several physical processes of interest. In the case of the Objectivity-based data store, a pointer collection is created for most of the physics tags, which allows for much faster retrieval of events that are of interest to a particular analysis. In the case of the ROOT-based KANGA data store, events with several related physics tags are groupped into "skim streams", which are then distributed to the interested institutions. 
The skims used in our analysis are BCCC3body and BCCKs3body, which, in KANGA, are part of the skim stream \#14. The events processed by the skim module are the events that had previously been tagged as multihadron events by the background filter (tag BGFMultiHadron). The selected events are required to contain three candidates that possess a combined 4-momentum translating into $|\Delta E|<0.45 \mathrm{GeV}$ and $\mid \sqrt{s} / 2-$ $m_{\mathrm{ES}} \mid<0.1 \mathrm{GeV}$ : for BCCC3body, three candidates from the GoodTracksLoose ${ }^{1}$ list ( $\pi^{ \pm}$and $K^{ \pm}$mass hypotheses are tested); for BCCKs3body, two candidates from the GoodTracksLoose list and one from the KsDefault list. In addition, selected events are required to contain at least four ChargedTracks, and the total energy in the event is required not to exceed $20 \mathrm{GeV}$.

\subsection{Reconstruction of $B$ meson candidates}

Our ntuple-producing code reads skimmed events and cycles over all possible combinations of GoodTracksLoose and KsDef ault candidates that are appropriate for the decay topology being looked at. For $B^{ \pm} \rightarrow \phi h^{ \pm}\left(\phi \rightarrow K^{+} K^{-}\right)$, the first member of the triplet of $B$ daughter candidates is required to have a charge of +1 , and the second member is required to have a charge of -1 . For $B^{0} \rightarrow \phi K^{0}\left(\phi \rightarrow K^{+} K^{-}, K^{0} \rightarrow K_{S}^{0} \rightarrow \pi^{+} \pi^{-}\right)$, the $K_{S}^{0}$ is the third member of the triplet.

The $B$ candidate selection process is optimized for speed and consists of several steps. The first step is checking whether the combined 4-momentum of the triplet trans-

\footnotetext{
${ }^{1}$ See Section 4.2 on page 57 for list definitions.
} 
lates into $|\Delta E|<0.35 \mathrm{GeV}$ and $\left(m_{\mathrm{ES}}-\sqrt{s} / 2\right)<0.14 \mathrm{GeV}$.

During the second step, a full fit of the entire $B$ candidate decay tree is performed using the GeoKin [79] vertex fitter with the geometric and $K_{S}^{0}$ mass constraints applied. We accept $B$ meson candidates with $|\Delta E|<0.3 \mathrm{GeV}$ and $\left(m_{\mathrm{ES}}-\sqrt{s} / 2\right)<0.1 \mathrm{GeV}$ and discard candidates for which the vertexing algorithm fails to converge.

Fig. 6.1 uses BABAR's WIRED event display [65] to illustrate what a reconstructed $B^{0} \rightarrow \phi K_{S}^{0}\left(K_{S}^{0} \rightarrow \pi^{+} \pi^{-}\right)$event may look like.

\subsection{Selection of events}

The following cuts were used in all stages of the analysis following the production of ntuples:

- $\left|\cos \theta_{T}\right|<0.9$ (see page 47 for discussion);

- number of GoodTracksVeryLoose tracks in the event $\geq 5$, the primary motivation being suppression of $\tau^{+} \tau^{-}$and $e^{+} e^{-} \rightarrow e^{+} e^{-} \gamma \gamma$ backgrounds;

- in $\phi \rightarrow K^{+} K^{-}, K^{ \pm}$candidates are required to satisfy criteria of PidKaonSMSSelector in its loosest mode, notApion (tighter $K^{ \pm}$PID is not indicated because $\phi$ is a narrow resonance and the background is small, so tightening the PID requirements leads to an increased signal-to-background ratio);

- all charged tracks that are part of the $B$ candidate decay tree, except for pions in $K_{S}^{0} \rightarrow \pi^{+} \pi^{-}$, are required to fail PidLHElectronSelector [92] in its tightest 


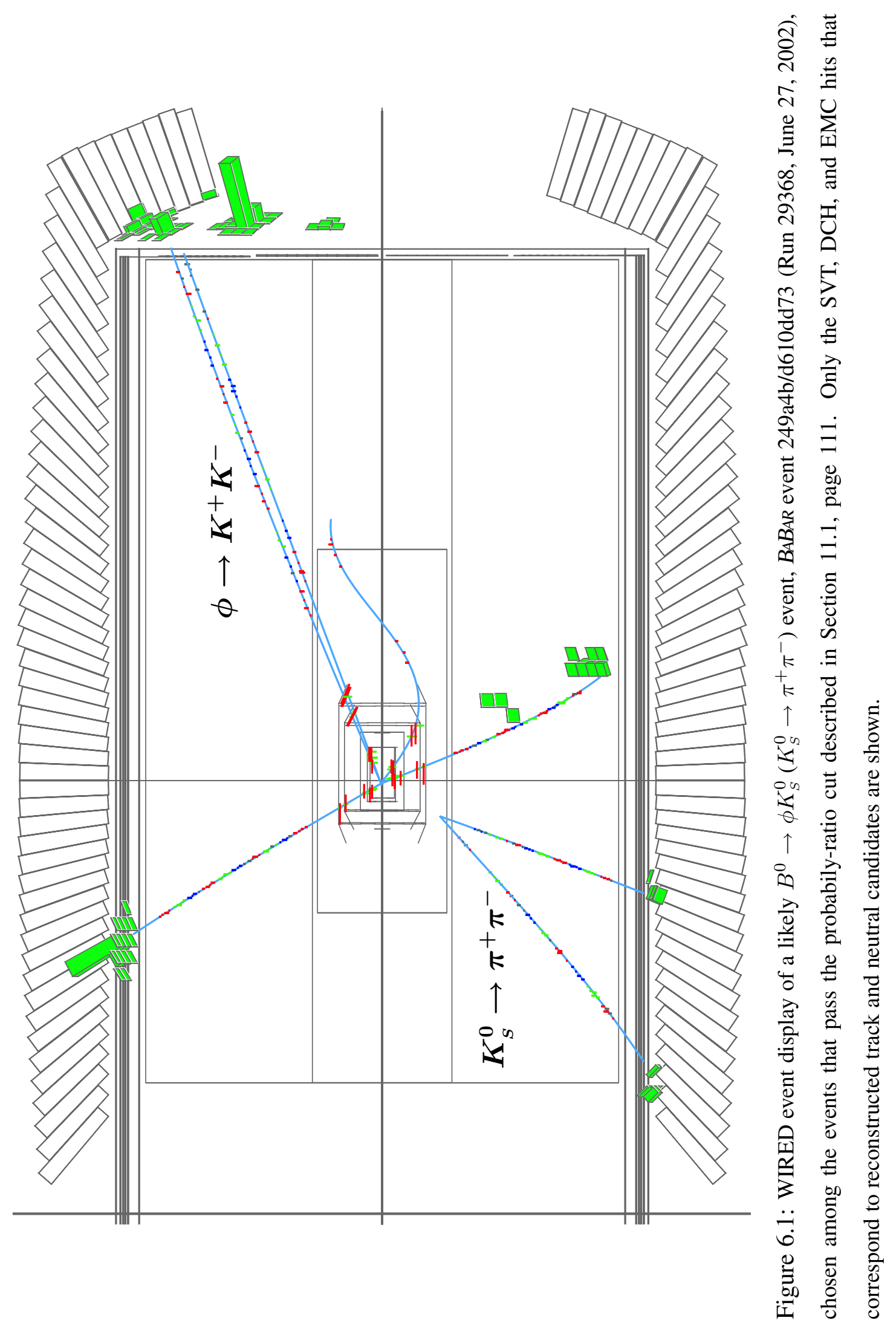


mode (tight), i.e., are required to be inconsistent with being electrons;

- $|\Delta E|<0.2 \mathrm{GeV}$ (for either the $K^{ \pm}$or the $\pi^{ \pm}$bachelor track mass hypothesis, if applicable);

- $5.20<m_{\mathrm{ES}}<5.30 \mathrm{GeV}$;

- The excluded region for sideband studies is $|\Delta E|<0.100 \mathrm{GeV}$ and $5.27<$ $m_{\mathrm{ES}}<5.30 \mathrm{GeV}$ (the $m_{\mathrm{ES}}$ and $\Delta E$ cuts are illustrated in Fig. 8.1, page 8.1);

- A very clean sample of $K_{S}^{0}$ candidates is obtained by applying the following optimized cuts:

$\star\left|m_{\pi^{+} \pi^{-}}-m_{K_{S \mathrm{PDG}}^{0}}\right|<0.012 \mathrm{GeV}$ (this is a rather loose cut: the core of the $m_{K_{S}^{0}}$ distribution is a Gaussian with $\sigma_{\text {core }} \approx 2.7 \mathrm{MeV}$, and the tails, which contain a few percent of the $K_{S}^{0}$ s, have $\sigma_{\text {tail }} \sim 6 \mathrm{MeV}$ );

$\star$ significance of the $K_{S}^{0}$ flight length $\ell / \sigma_{\ell}>3$;

$\star$ cosine of the angle between $K_{S}^{0}$ momentum and its line-of-flight (the vector connecting the reconstructed $B$ and $K_{S}^{0}$ decay vertices) $\cos \alpha>0.995$.

In addition, the following cuts were used in $B \rightarrow D \pi$ calibration-mode studies:

- “CLEO” Fisher discriminant $\mathcal{F}<1.3$ (see page 50 for discussion);

- in $B \rightarrow \pi^{ \pm} D, \bar{D}^{0} \rightarrow K^{+} \pi^{-}, \bar{D}^{0} \rightarrow K^{+} K^{-}, \bar{D}^{0} \rightarrow \pi^{+} \pi^{-}, D^{-} \rightarrow K_{S}^{0} \pi^{-}, K^{ \pm}$ candidates are required to satisfy criteria of PidKaonSMSSelector in its loosest mode, notApion, and $\pi^{ \pm}$candidates, except those in $K_{S}^{0} \rightarrow \pi^{+} \pi^{-}$, to fail criteria of PidKaonSMSSelector [80] in its tight mode, i.e., are required to $i$ be 
inconsistent with being kaons (no tighter PID is necessary because these modes are very clean);

- all charged tracks that are part of the $B$ candidate decay tree, except for pions in $K_{S}^{0} \rightarrow \pi^{+} \pi^{-}$, are required to fail PidProtonLHSelector [93] in its tightest mode (veryTight);

- $\left|m_{D_{\text {cand }}}-m_{D_{\mathrm{PDG}}}\right|<0.0142 \mathrm{GeV}(\mathrm{a} \sim 2 \sigma$ cut $)$;

- The signal region in calibration-mode studies is $|\Delta E|<0.040 \mathrm{GeV}$ and $\mid m_{\mathrm{ES}}-$ $5.279 \mid<0.0054 \mathrm{GeV}\left(\mathrm{a} \sim 2 \sigma\right.$ cut in both $\Delta E$ and $\left.m_{\mathrm{ES}}\right)$.

The following additional cuts are applied to $B$ candidates used in the maximumlikelihood (ML) analysis:

- in $\phi \rightarrow K^{+} K^{-}, 0.990<m_{K^{+} K^{-}}<1.050 \mathrm{GeV}$;

- bachelor charged tracks must be associated with $>5$ Cherenkov photons in the DIRC (a standard ring reconstruction quality cut) and have a Cherenkov angle pull of $<4.0$ for either the $K^{ \pm}$or the $\pi^{ \pm}$hypothesis;

- the value of the Fisher discriminant is required to be within $4 \sigma$ of either the signal or the continuum background hypotheses. This cut provides us with protection against poorly reconstructed events that may have a value of $\mathcal{F}$ many sigmas away from its true value in the direction of the signal. Presence of such events would adversely affect the overall likelihood and bias the fit result toward an inflated number of signal events; 
- all non-bachelor charged tracks that are part of the $B$ candidate decay tree, except for pions in $K_{S}^{0} \rightarrow \pi^{+} \pi^{-}$, are required to fail PidProtonLHSelector in its tightest mode (veryTight). We cannot apply proton veto to the bachelor track because the proton selector makes heavy use of the charged track's Cherenkov angle information, one of the input variables of the ML fit. 


\section{Chapter 7}

\section{Calibration Modes}

Study of calibration modes, i.e., modes with relatively large event yields that have topologies similar to the modes that are the primary focus of our analysis, is an essential tool that allows us to investigate and understand possible discrepancies between data and Monte Carlo and make necessary adjustments to our analysis to accomodate them.

The calibration mode most appropriate for the study of $B^{ \pm} \rightarrow \phi h^{ \pm}\left(\phi \rightarrow K^{+} K^{-}\right)$is $B^{+} \rightarrow \pi^{+} \bar{D}^{0}\left(\bar{D}^{0} \rightarrow K^{+} \pi^{-}\right)$, which has a very similar final state (three charged tracks, albeit of a different PID content) and a branching fraction of $\sim 2.0 \times 10^{-4}$, which is approximately 40 times the previously measured branching fraction of $B^{ \pm} \rightarrow \phi K^{ \pm}$, $\phi \rightarrow K^{+} K^{-}$. We have also looked, with only $56.3 \mathrm{fb}^{-1}$, at two other $B^{+} \rightarrow \pi^{+} \bar{D}^{0}$ modes with three charged tracks in the final state: $B^{+} \rightarrow \pi^{+} \bar{D}^{0}\left(\bar{D}^{0} \rightarrow K^{+} K^{-}\right)$ $\left(\mathcal{B} \sim 2.3 \times 10^{-5}\right)$ and $B^{+} \rightarrow \pi^{+} \bar{D}^{0}\left(\bar{D}^{0} \rightarrow \pi^{+} \pi^{-}\right)\left(\mathcal{B} \sim 0.8 \times 10^{-5}\right)$.

The only calibration mode with a decay topology somewhat similar to that in $B^{0} \rightarrow$ 

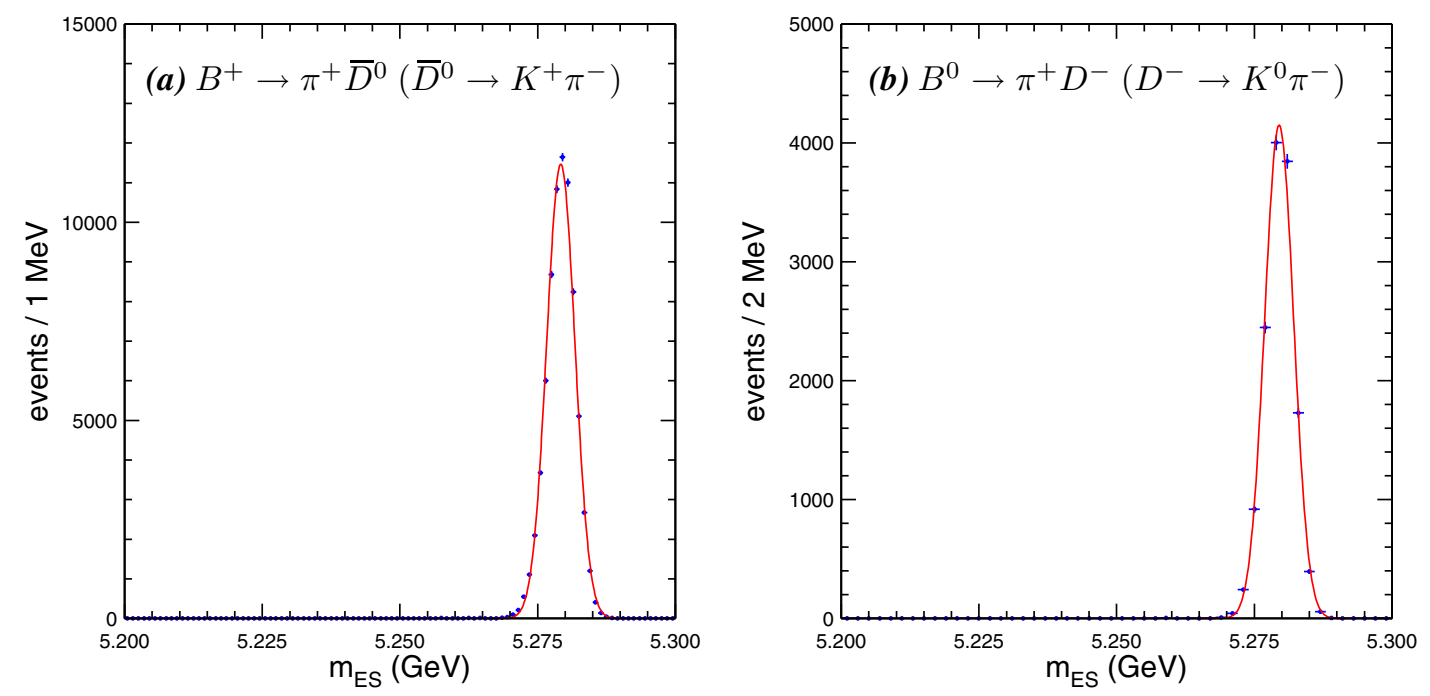

Figure 7.1: $m_{\mathrm{ES}}$ distributions for signal Monte Carlo, (a) $B^{+} \rightarrow \pi^{+} \bar{D}^{0}\left(\bar{D}^{0} \rightarrow K^{+} \pi^{-}\right)$, and (b) $B^{0} \rightarrow \pi^{+} D^{-}\left(D^{-} \rightarrow K^{0} \pi^{-}\right)$.

$\phi K^{0}\left(\phi \rightarrow K^{+} K^{-}\right)$and a suitably high branching fraction is $B^{0} \rightarrow \pi^{+} D^{-}\left(D^{-} \rightarrow\right.$ $\left.K^{0} \pi^{-}\right)$

\section{$7.1 m_{\mathrm{ES}}$}

In the calibration mode studies, we fit the $m_{\mathrm{ES}}$ distributions in signal Monte Carlo with a single Gaussian (Fig. 7.1), and the $m_{\mathrm{ES}}$ distributions in data with a sum of a single Gaussian and an ARGUS-shaped continuum background component (Fig. 7.2). Fit results are presented in Table 7.1.

We observe a shift of $+0.65 \pm 0.04 \mathrm{MeV}$ between the mean values of the $m_{\mathrm{ES}}$ distributions in data and Monte Carlo in the $B^{+} \rightarrow \pi^{+} \bar{D}^{0}\left(\bar{D}^{0} \rightarrow K^{+} \pi^{-}\right)$mode and a shift of $+0.84 \pm 0.10 \mathrm{MeV}$ in $B^{0} \rightarrow \pi^{+} D^{-}\left(D^{-} \rightarrow K^{0} \pi^{-}\right)$; these are the corrections 

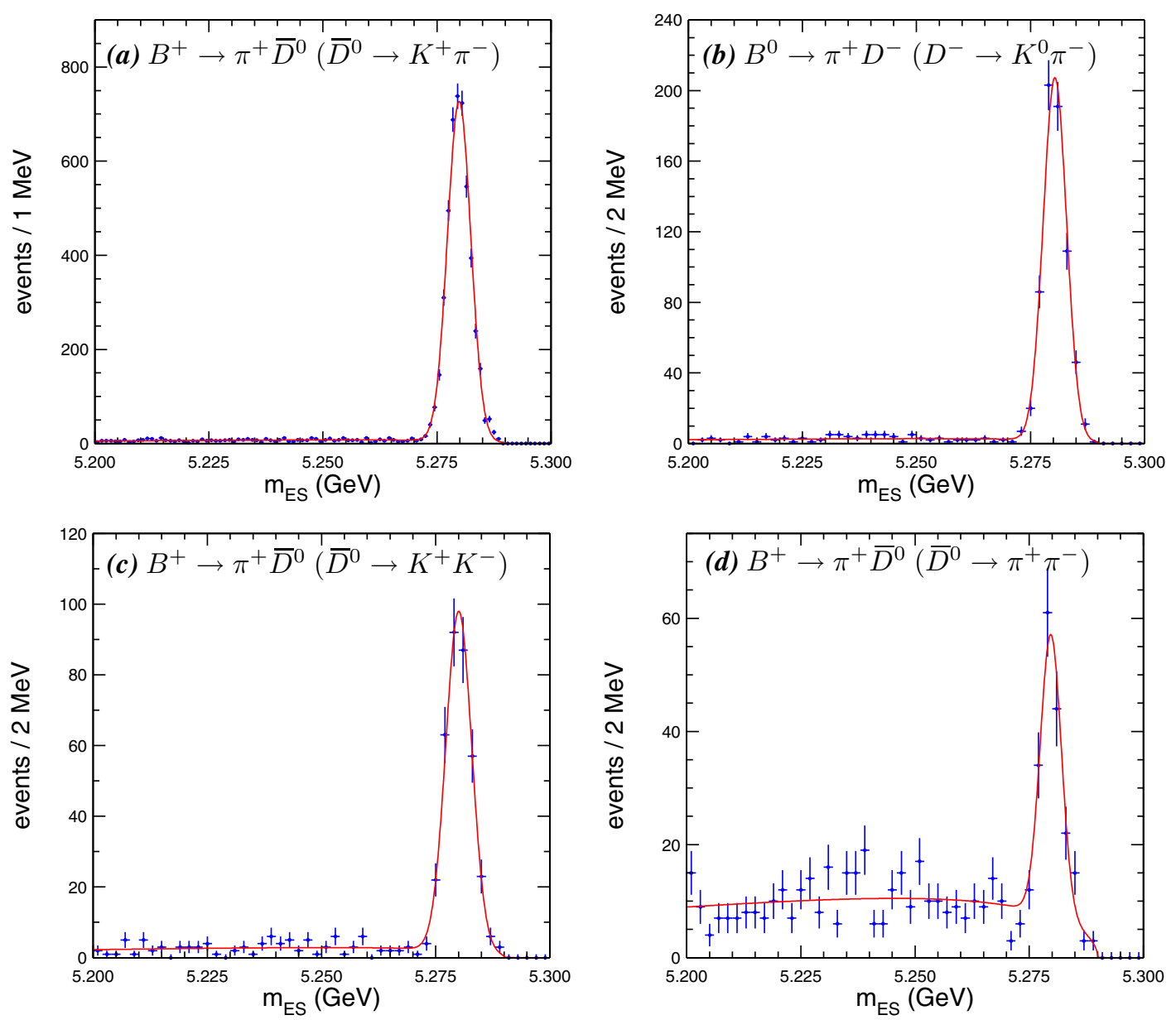

Figure 7.2: $m_{\mathrm{ES}}$ distributions in the on-resonance signal region, (a) $B^{+} \rightarrow \pi^{+} \bar{D}^{0}\left(\bar{D}^{0} \rightarrow\right.$ $\left.K^{+} \pi^{-}\right)$, (b) $B^{0} \rightarrow \pi^{+} D^{-}\left(D^{-} \rightarrow K^{0} \pi^{-}\right),(c) B^{+} \rightarrow \pi^{+} \bar{D}^{0}\left(\bar{D}^{0} \rightarrow K^{+} K^{-}\right)$, and $(d) B^{+} \rightarrow$ $\pi^{+} \bar{D}^{0}\left(\bar{D}^{0} \rightarrow \pi^{+} \pi^{-}\right)$. The full Summer 2002 data set $\left(81.9 \mathrm{fb}^{-1}\right)$ was used in the making of plots $(a)$ and $(b)$, while plots $(c)$ and $(d)$ reflect only the Winter 2001 data set $\left(56.3 \mathrm{fb}^{-1}\right)$. 


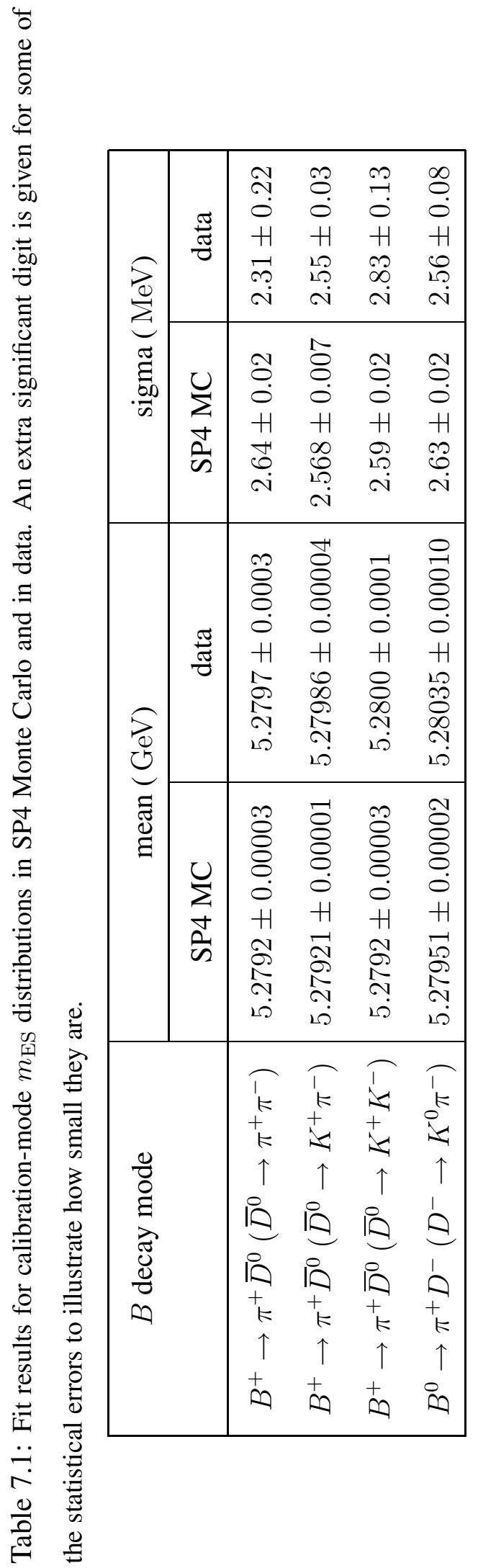


to the $m_{\mathrm{ES}}$ means that we will later apply to the values observed in $B^{ \pm} \rightarrow \phi h^{ \pm}$and $B^{0} \rightarrow \phi K_{S}^{0}$ signal MC. We do not see a statistically significant difference between the values of $\sigma_{m_{\mathrm{ES}}}$ in data and Monte Carlo.

We also observe statistically significant differences between the mean values of $m_{\mathrm{ES}}$ in $B^{0}$ and $B^{ \pm}$decays, $+0.30 \pm 0.03 \mathrm{MeV}$ in $\mathrm{MC}$ and $+0.49 \pm 0.11 \mathrm{MeV}$ in data, which is consistent with our expectations based on the difference between the values of $B^{0}$ and $B^{ \pm}$masses used in SP4 Monte Carlo production $\left(m_{B^{0}{ }_{\mathrm{PDG}}}=5279.4 \pm 0.5 \mathrm{MeV}\right.$, $\left.m_{B^{ \pm}}{ }_{\mathrm{PDG}}=5279.0 \pm 0.5 \mathrm{MeV}\right)[8]$ and the PDG value of $\left(m_{B^{0}}-m_{B^{ \pm}}\right)_{\mathrm{PDG}}=+0.33 \pm$ $0.28 \mathrm{MeV}$ [9]. Please note that we are not trying to claim a measurement of the $m_{B^{ \pm}}-$ $m_{B^{0}}$ mass difference that is better than the PDG value because we do not have (and, for the purposes of our analysis, do not need) an understanding of the systematics involved in the determination of $m_{\mathrm{ES}}$ in $B A B A R$ at the level required to make such a measurement possible; for the purpose of determining the systematic errors on the $B \rightarrow \phi h$ branching fractions, we assign conservative errors of $\pm 0.3 \mathrm{MeV}$ to the $m_{\mathrm{ES}}$ means and $\pm 0.1 \mathrm{MeV}$ to the $m_{\mathrm{ES}}$ distribution widths.

\section{2 $\Delta E$ and the tracking bias in runs 18000-22929}

With the exception of 84 runs that were (re)processed after October 15, 2001, all runs in the range 18000-22929 suffer from a Drift Chamber time-to-distance calibration problem, which results in a charge-dependent bias in the momenta of all charged tracks. It turns out that the only variable in our analysis that is measurably affected by this tracking 

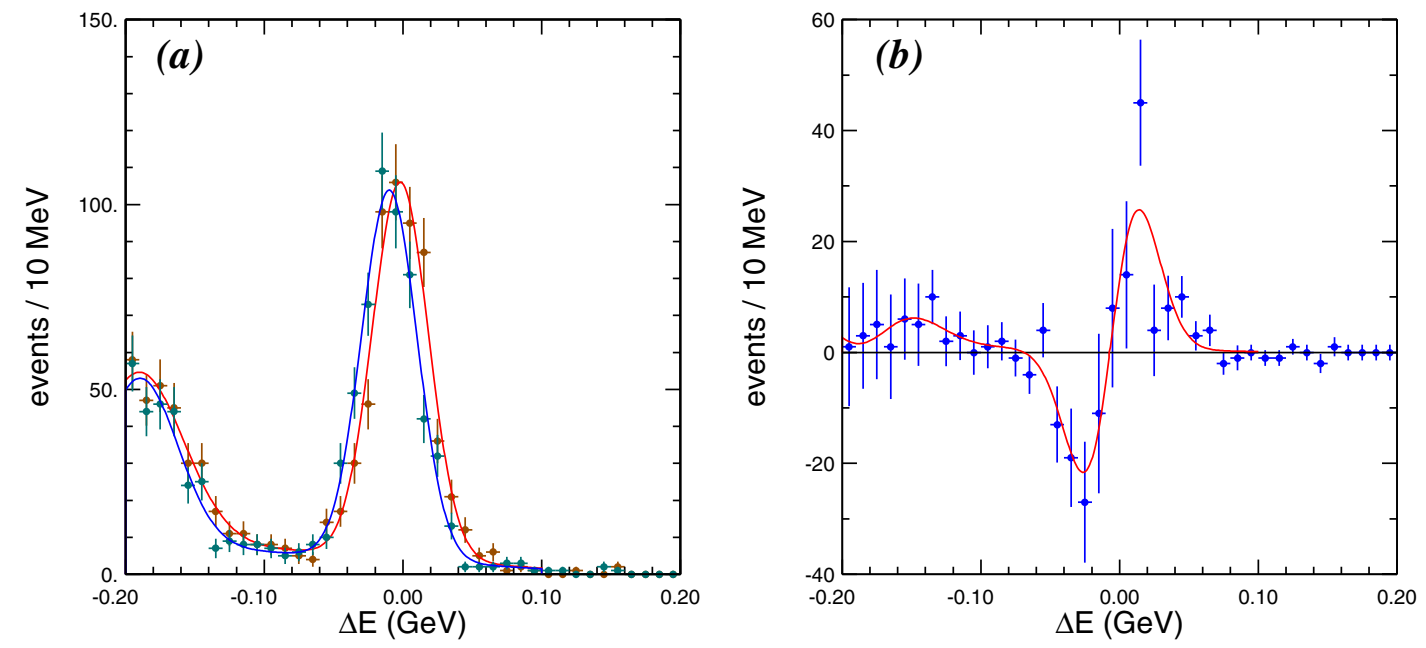

Figure 7.3: (a) Uncorrected $\Delta E$ distributions for positively (right curve) and negatively (left curve) charged $B^{+} \rightarrow \pi^{+} \bar{D}^{0}\left(\bar{D}^{0} \rightarrow K^{+} \pi^{-}\right)$, c.c., candidates in data, and $(b)$ the difference of these two distributions.

bias is $\Delta E$ - this is why the discussion of this problem takes place in this section.

The tracking bias in the 2001 data was first noticed in the Spring of 2001 and fixed in data processed by OPR or reprocessed after October 16, 2001; most of the runs in the range 18000-22929 are affected. The first analysis taking this bias into account and correcting for it was the David Williams' analysis of $D^{0}$ branching fractions and mixing $[84,95]$. More recently, a discussion of this bias took place on the Tracking Reconstruction Software hypernews forum [66]; the most comprehensive discussion can be found in [67].

It has been shown that the effect of the bias can be approximately modelled by applying the correction

$$
q / p_{t \text { corrected }}=q / p_{\text {traw }}+C \times p_{t \text { raw }}
$$


while keeping the direction of the momentum unchanged. Rewriting the above expression,

$$
p_{\text {corrected }}=p_{\text {raw }} \times\left(1-C \times q \times\left(p_{\text {traw }}\right)^{2}\right) .
$$

Here $q$ is the track's charge, $p$ is the magnitude of its 3 -momentum, and $p_{t}$ is its transverse momentum. Several approaches have been used to determine the value of the constant $C$, and it has been found that $C=(+3.4 \pm 0.7) \times 10^{-4}[67]$.

According to Eq. 7.2, for a given polar angle, the magnitude of the momentum bias is proportional to the cube of the track's momentum. This means that in decays $B \rightarrow X h^{ \pm}$ the bulk of the charge-dependent $\Delta E$ bias will be due to the bachelor track. Depending on the charge of the bachelor track, $\Delta E$ distributions will be shifted in opposite directions and slightly broadened, the split being $7.89 \pm 1.45 \mathrm{MeV}$ in $B^{+} \rightarrow \pi^{+} \bar{D}^{0}\left(\bar{D}^{0} \rightarrow\right.$ $K^{+} \pi^{-}$) (determined from data, Fig. 7.3) and reaching $11.8 \pm 2.2 \mathrm{MeV}$ in $B^{+} \rightarrow \pi^{+} \pi^{0}$ (our extrapolation of the previous number). Note that since the kinetic energy available in the decay $\phi \rightarrow K^{+} K^{-}$is very small, the two oppositely-charged kaons are nearly collinear, so no correction of their momenta is necessary. Similarly, the bias due to the tracks from $K_{S}^{0} \rightarrow \pi^{+} \pi^{-}$decays is also very small.

Our approach is to apply the correction in Eq. 7.2 to all bachelor tracks, then use Eq. 4.2 to determine the shift of $\Delta E$ this correction causes, and, finally, adjust $\Delta E$ of the kinematically-fitted version of our $B$ candidate by the amount of this shift.

In the calibration-mode studies, we fit $\Delta E$ distributions in signal Monte Carlo with a 

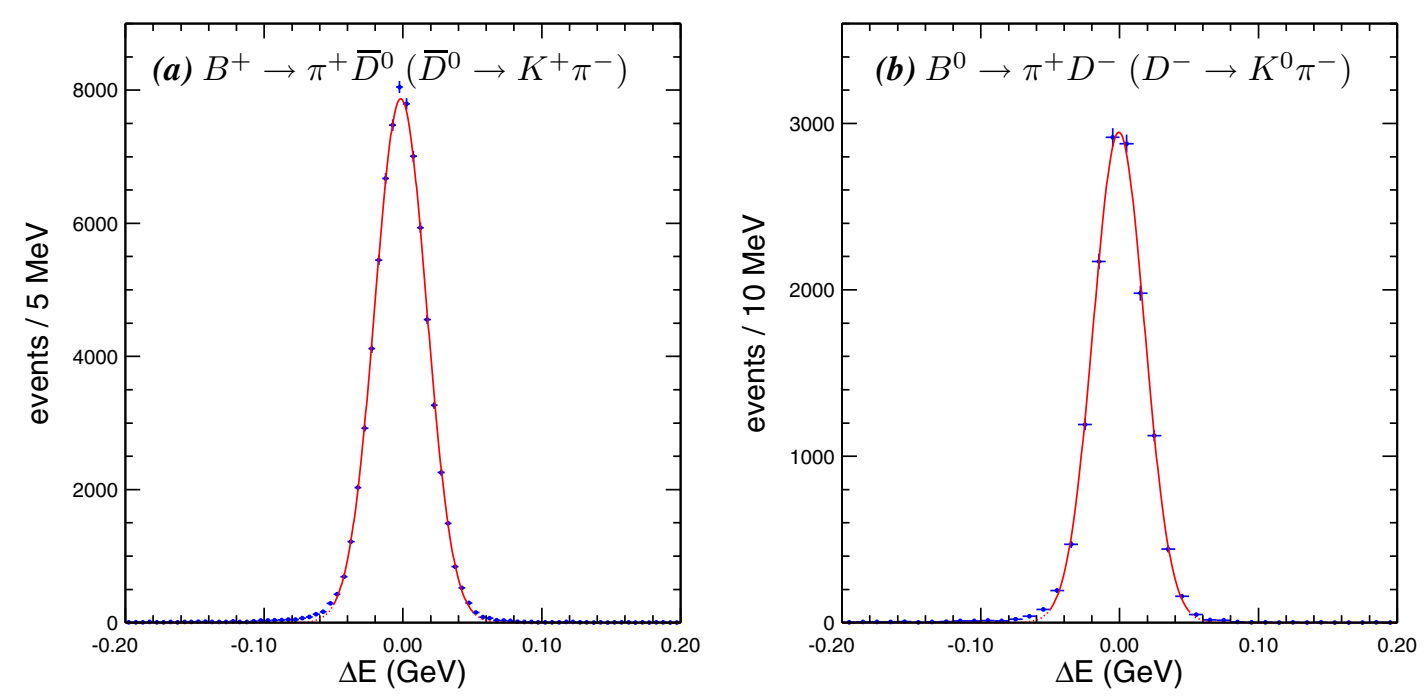

Figure 7.4: $\Delta E$ distributions for signal Monte Carlo, (a) $B^{+} \rightarrow \pi^{+} \bar{D}^{0}\left(\bar{D}^{0} \rightarrow K^{+} \pi^{-}\right)$, and (b) $B^{0} \rightarrow \pi^{+} D^{-}\left(D^{-} \rightarrow K^{0} \pi^{-}\right)$.

single Gaussian (Fig. 7.4), and $\Delta E$ distributions in data with a sum of a single Gaussian, a first-degree polynomial to describe continuum background, and a second Gaussian to describe background from $B \rightarrow D^{*} X$ modes (Fig. 7.5). The fit results are presented in Table 7.2.

In $B^{+} \rightarrow \pi^{+} \bar{D}^{0}\left(\bar{D}^{0} \rightarrow K^{+} \pi^{-}\right)$, the calibration mode with three charged tracks in the final state, we observe a $\Delta E$ shift in data with respect to simulation of $-3.8 \pm 0.3$ $\mathrm{MeV}$; to account to the possible systematic errors due to the different flavor content and momenta distributions in the $B^{+} \rightarrow \pi^{+} \bar{D}^{0}\left(\bar{D}^{0} \rightarrow K^{+} \pi^{-}\right)$and $B^{ \pm} \rightarrow \phi h^{ \pm}$final states, we assign a conservative error of $\pm 2 \mathrm{MeV}$ to this correction. The $\Delta E$ shift observed in the mode with two charged tracks and a $K_{S}^{0}$ in the final state, $B^{0} \rightarrow \pi^{+} D^{-}\left(D^{-} \rightarrow\right.$ $\left.K^{0} \pi^{-}\right)$, is $-4.7 \pm 0.9 \mathrm{MeV}$; we use it in the $B^{0} \rightarrow \phi K_{S}^{0}$ analysis, with the error being also set to $\pm 2 \mathrm{MeV}$ for the purpose of systematics studies. 

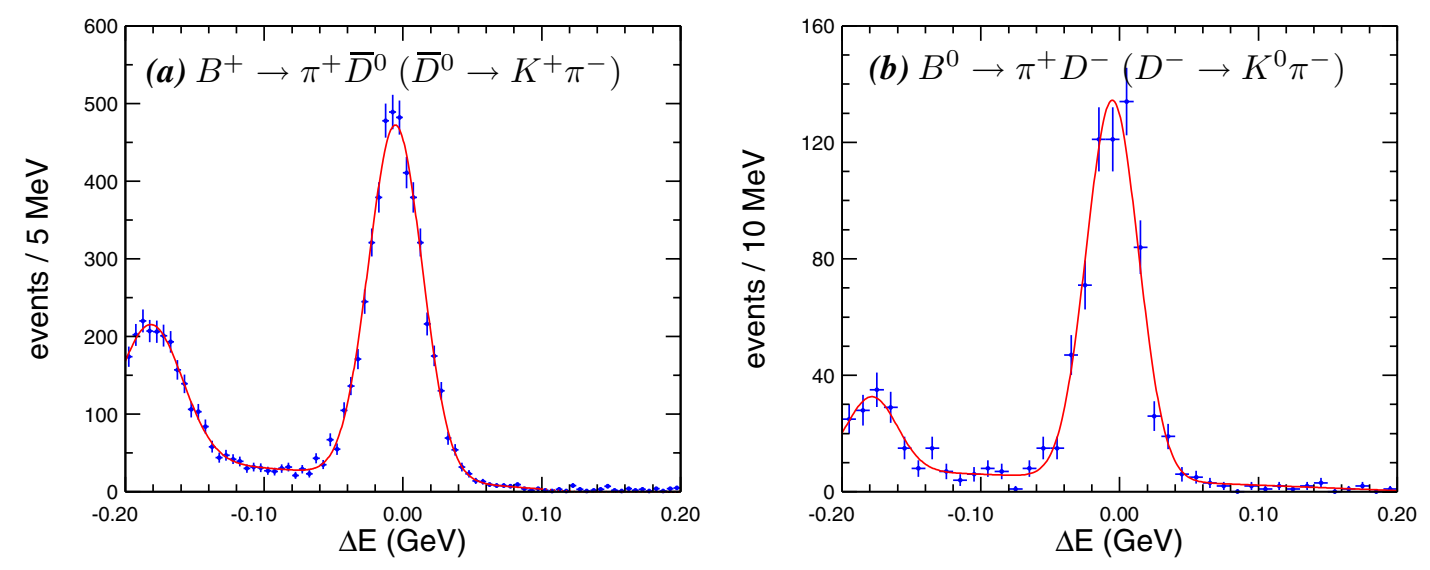

Figure 7.5: $\Delta E$ distributions in the on-resonance signal region, (a) $B^{+} \rightarrow \pi^{+} \bar{D}^{0}\left(\bar{D}^{0} \rightarrow\right.$ $\left.K^{+} \pi^{-}\right)$, (b) $B^{0} \rightarrow \pi^{+} D^{-}\left(D^{-} \rightarrow K^{0} \pi^{-}\right)$.

We also observe that SP4 Monte Carlo underestimates $\sigma_{\Delta E}$ in data by $3.5 \pm 1.6 \%$ in $B^{+} \rightarrow \pi^{+} \bar{D}^{0}\left(\bar{D}^{0} \rightarrow K^{+} \pi^{-}\right)$; the correction in $B^{0} \rightarrow \pi^{+} D^{-}\left(D^{-} \rightarrow K^{0} \pi^{-}\right)$ is $1.8 \pm 4.1 \%$. We scale the $\sigma_{\Delta E}$ values observed in $B \rightarrow \phi h$ signal Monte Carlo appropriately and assign to them an error of $\pm 1 \mathrm{MeV}$.

\subsection{Fisher Discriminant $(\mathcal{F})$}

There exists a variety of reasons that may potentially lead to a discrepancy between $\mathcal{F}$ distributions in data and Monte Carlo: imperfectly simulated backgrounds, dead electronics channels, etc. We compare $\mathcal{F}$ distributions in SP4 MC and data for $B^{+} \rightarrow$ $\pi^{+} \bar{D}^{0}\left(\bar{D}^{0} \rightarrow K^{+} \pi^{-}\right)$, the highest-statistics calibration mode, and observe no statistically significant discrepancies (Fig. 7.6). Although the continuum background in this mode is very small, as evidenced in Fig. 7.2(a), for maximum purity the $\mathcal{F}$ distribution for the $B^{+} \rightarrow \pi^{+} \bar{D}^{0}\left(\bar{D}^{0} \rightarrow K^{+} \pi^{-}\right)$signal in data was obtained by subtracting with 


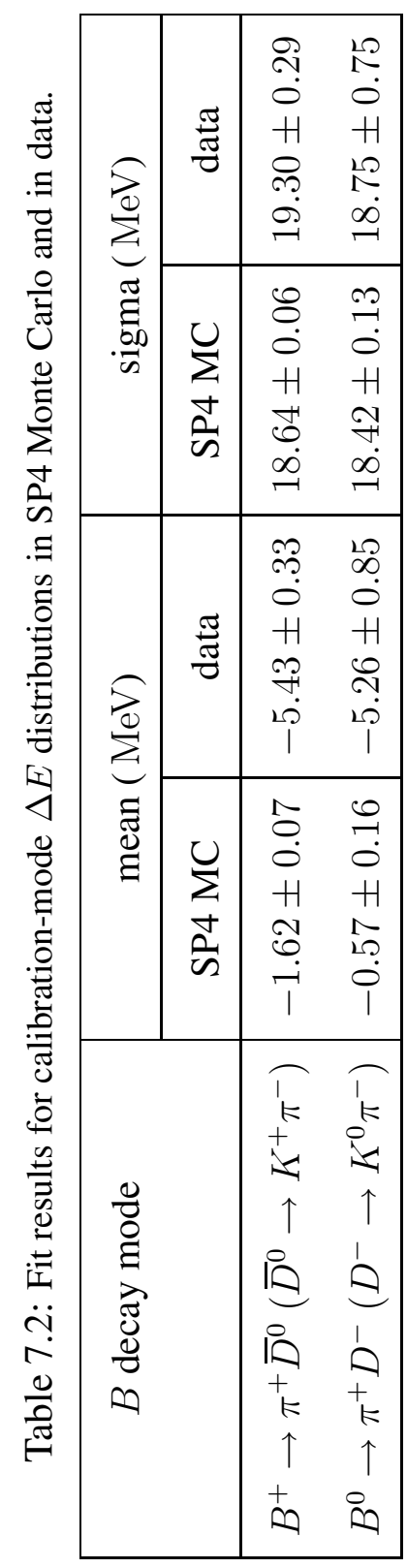




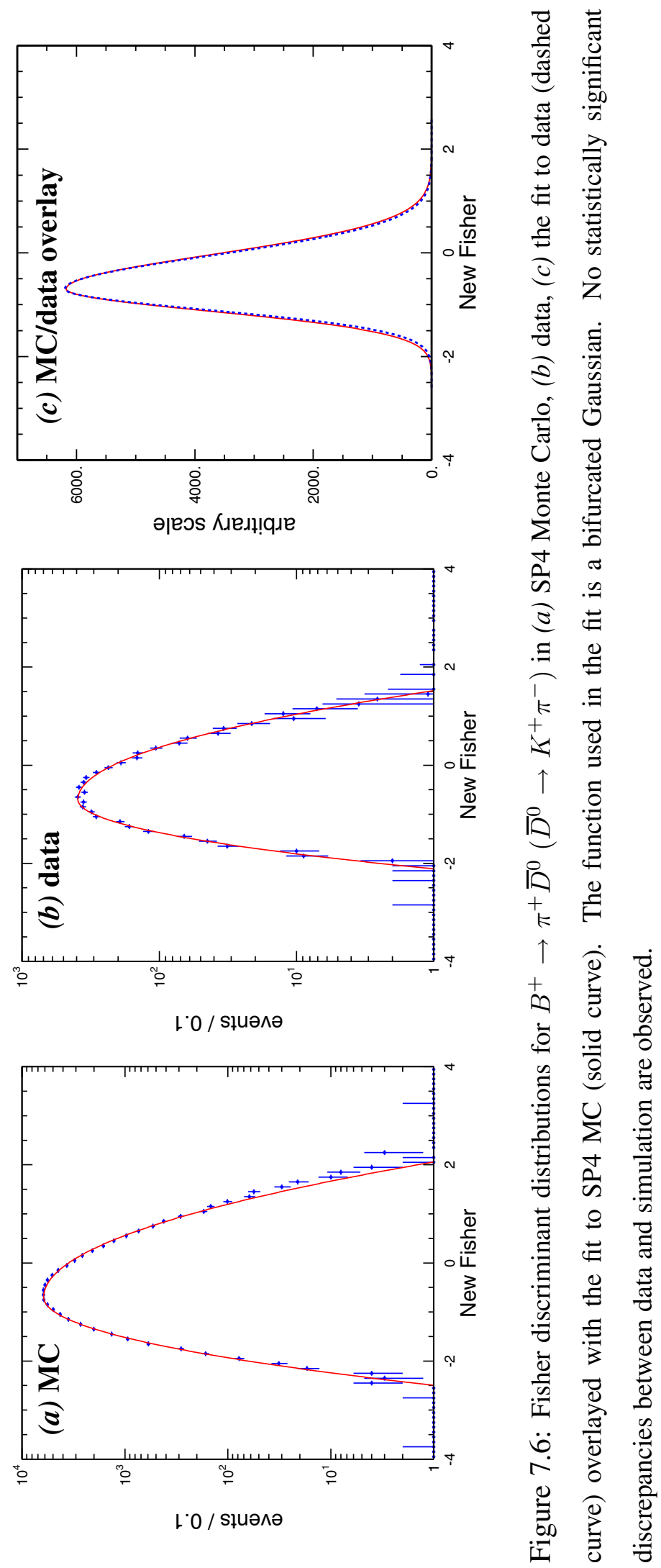


a proper weight the $\mathcal{F}$ distribution in the $m_{\mathrm{ES}}$ sideband from the $\mathcal{F}$ distribution in the signal box. 


\section{Chapter 8}

\section{Probability Distribution Functions}

The principal and most critical job in a maximum-likelihood analysis is accurate determination of the probability density functions (PDFs) of all quantities used as input to the fit for both components of the fit, the signal and the continuum background.

Large samples of SP4 signal Monte Carlo (45000 events for $B^{ \pm} \rightarrow \phi K^{ \pm}$and 44000

events for $B^{0} \rightarrow \phi K_{S}^{0}$ ) are used to determine signal PDFs; on-resonance data sideband appropriately chosen to avoid potential signal events (see Fig. 8.1) is used to determine PDFs for the continuum background. We do not use off-resonance data in determination of continuum background PDFs because in BABAR the size of the off-resonance data set is only $\sim 1 / 8$ of the size of the on-resonance data set; we used off-resonance data only for cross-checks at the early stages of this analysis. Please refer to page 65 for the list of cuts used in the making of the PDFs.

Modes with a charged bachelor track present an additional complication: the possible dependence of continuum background PDFs on the flavor of the bachelor particle, 


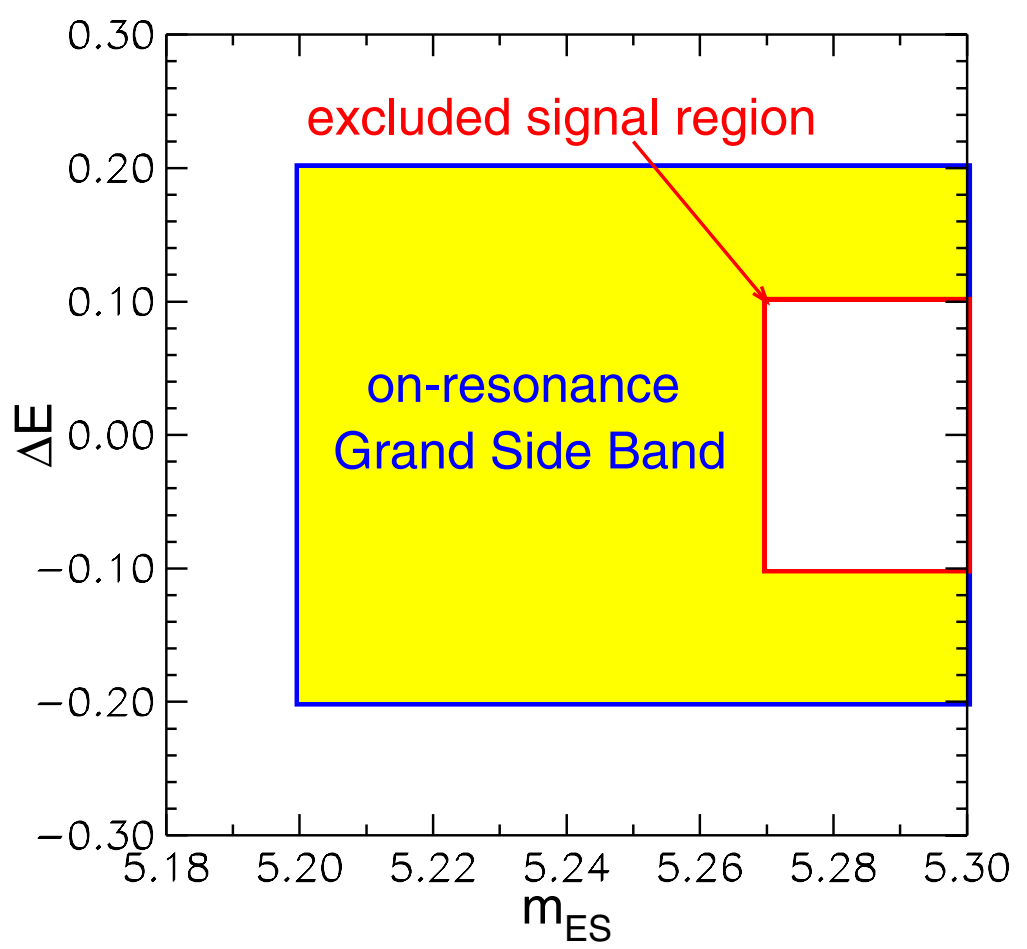

Figure 8.1: Definition of the on-resonance "grand" sideband in $m_{\mathrm{ES}}$ and $\Delta E$.

$\pi^{ \pm}$or $K^{ \pm}$. Until these become high-statistics analyses, we can keep using unified PDFs while factoring the $\pi^{ \pm} / K^{ \pm}$differences into errors on PDF parameters. This is our preferred option. Another possibility is not to do a simultaneous fit to $B^{ \pm} \rightarrow \phi K^{ \pm}$and $B^{ \pm} \rightarrow \phi \pi^{ \pm}$, but to look exclusively for $B^{ \pm} \rightarrow \phi K^{ \pm}\left(B^{ \pm} \rightarrow \phi \pi^{ \pm}\right.$is strongly suppressed in the Standard Model).

Here is the statistical procedure we use to determine PDF parameter errors in cases with a charged bachelor track. We determine three sets of PDF parameters: for the combined sample and for two samples with Cherenkov angle pull cuts applied, the " $\pi^{ \pm}$" sample being the sample in which $\left(\operatorname{pull}_{\pi}<\operatorname{pull}_{K} \& \operatorname{pull}_{\pi}<4\right.$ ), and similarly for the " $K^{ \pm}$" sample. For each PDF parameter used in the ML fit we take the mean $\mu_{\text {comb }}$ 

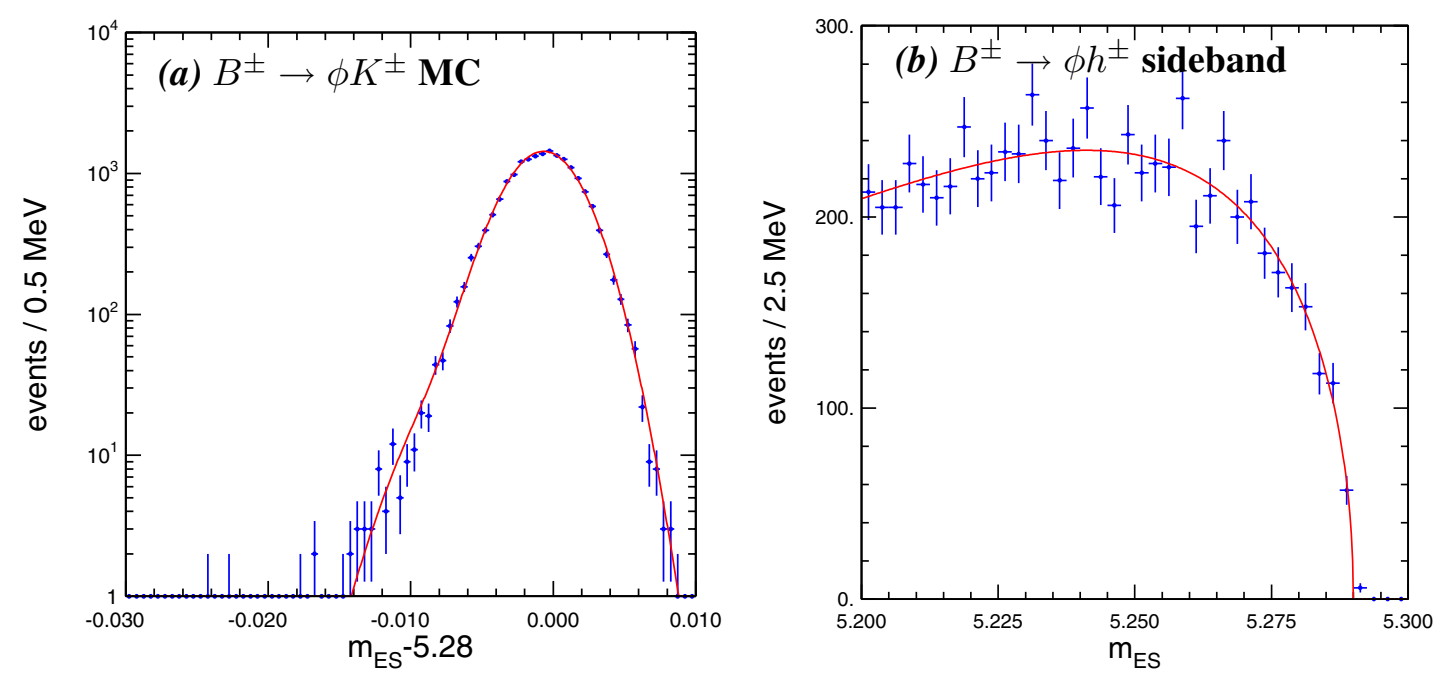

Figure 8.2: $m_{\mathrm{ES}}$ distributions for $(a) B^{ \pm} \rightarrow \phi K^{ \pm}$signal Monte Carlo, and $(b)$ the $B^{ \pm} \rightarrow \phi h^{ \pm}$ on-resonance sideband.

determined on the combined sample, and take the variance $\sigma^{2}$ to be the sum of the variance $\sigma_{\text {comb }}^{2}$ obtained on the combined sample and the variance of properly weighted central values of the " $\pi^{ \pm}$" and the " $K^{ \pm}$" samples:

$$
\sigma^{2}=\sigma_{\mathrm{comb}}^{2}+\frac{\frac{\left(\mu_{\pi}-\mu_{\mathrm{comb}}\right)^{2}}{\sigma_{\pi}^{2}}+\frac{\left(\mu_{K}-\mu_{\mathrm{comb}}\right)^{2}}{\sigma_{K}^{2}}}{\frac{1}{\sigma_{\pi}^{2}}+\frac{1}{\sigma_{K}^{2}}}
$$

\section{1 $m_{\mathrm{ES}}$}

We fit $m_{\mathrm{ES}}$ distributions in signal Monte Carlo samples with a double Gaussian, and use an ARGUS function (Eq. A.2) with the beam energy fixed at $5.2900 \mathrm{GeV}$ to fit $m_{\mathrm{ES}}$ distributions in the sideband above and below the excluded signal region (Fig. 8.2, Fig. 8.3). PDF parameters for MC (before corrections due to the calibration mode studies) and onresonance sidebands are given in Table 8.1. 

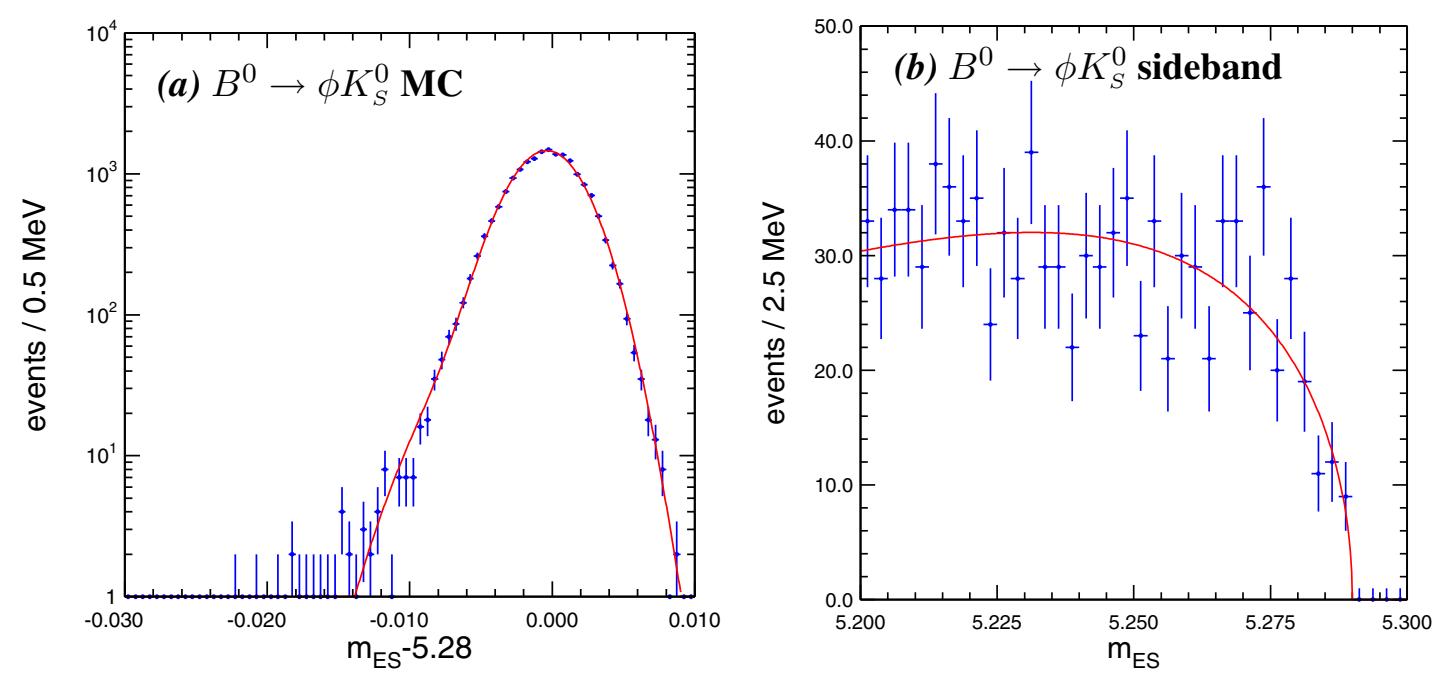

Figure 8.3: $m_{\mathrm{ES}}$ distributions for $(a) B^{0} \rightarrow \phi K_{S}^{0}$ signal Monte Carlo, and $(b)$ the $B^{0} \rightarrow \phi K_{S}^{0}$ on-resonance sideband. 


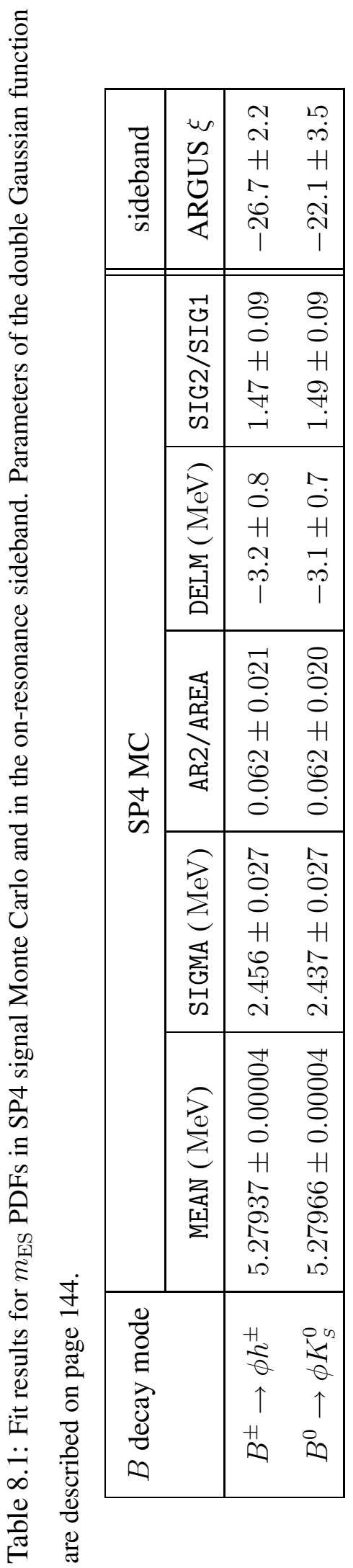



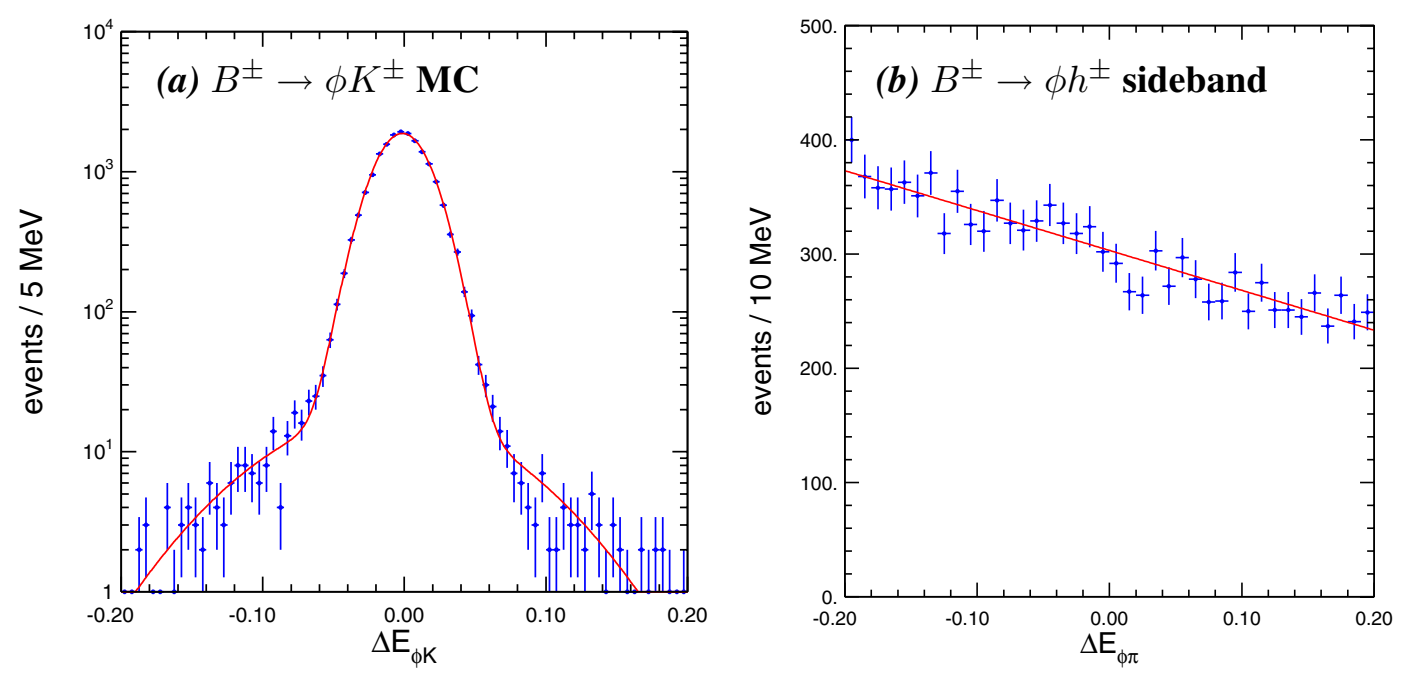

Figure 8.4: $\Delta E$ distributions for $(a) B^{ \pm} \rightarrow \phi K^{ \pm}$SP4 signal Monte Carlo, and (b) the $B^{ \pm} \rightarrow$ $\phi h^{ \pm}$on-resonance sideband.

\section{$8.2 \Delta E$}

Unlike $m_{\mathrm{ES}}, \Delta E$ depends on masses of final-state particles. There is no ambiguity in modes where the bachelor particle is a $K_{S}^{0}$; for modes with a bachelor charged track, we compute two values of $\Delta E$ for each $B$ candidate, one for a $\pi^{ \pm}$and the other for a $K^{ \pm}$mass hypothesis, and use both as input parameters of the maximum-likelihood fit, where $\Delta E_{K}$ is used to compute $\mathcal{P}_{K S}$ and $\Delta E_{\pi}$ is used to compute $\mathcal{P}_{\pi S}, \mathcal{P}_{\pi C}$ and $\mathcal{P}_{K C}$ (see page 60; [82], page 58).

We fit $\Delta E$ distributions in signal Monte Carlo samples with a double Gaussian, and use a first-degree polynomial to fit $\Delta E$ distributions in the sideband to the left of the excluded signal region (Fig. 8.4, Fig. 8.5). PDF parameters for MC (before corrections due to the calibration mode studies) and on-resonance sidebands are given in Table 8.2. 

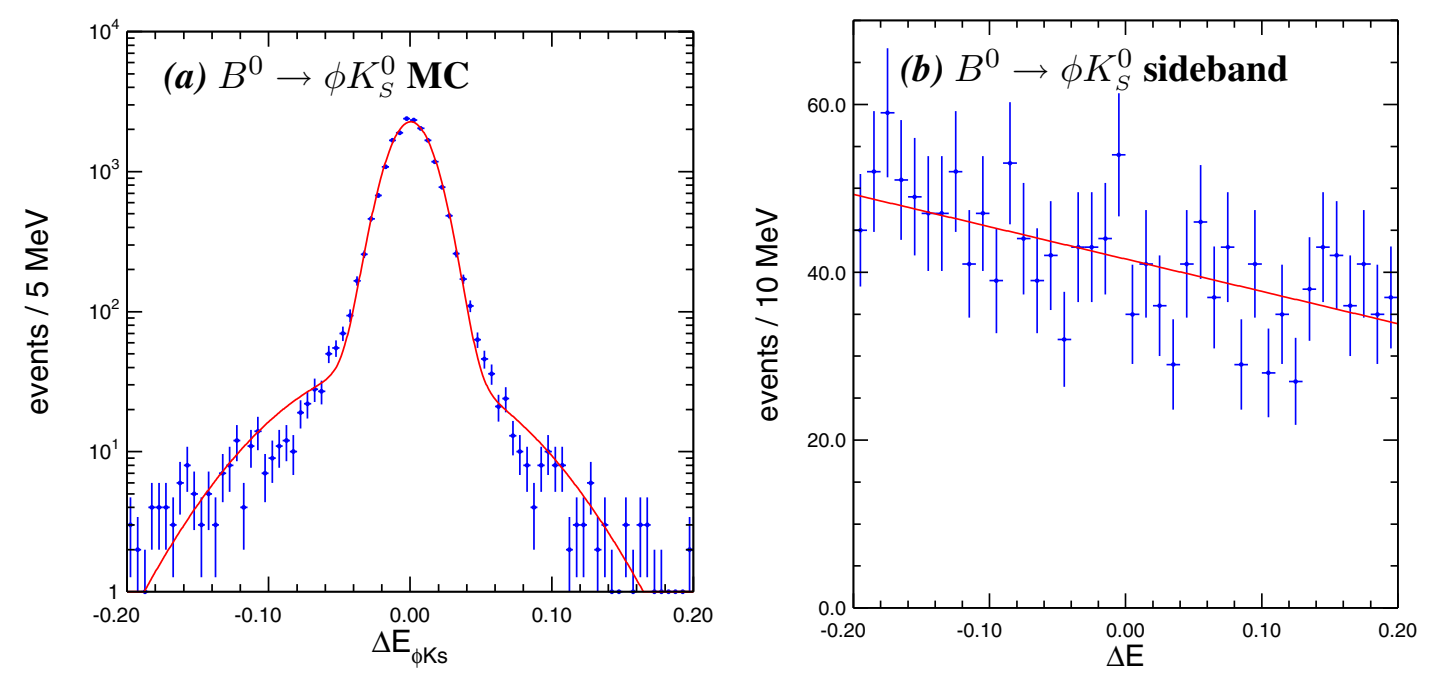

Figure 8.5: $\Delta E$ distributions for $(a) B^{0} \rightarrow \phi K_{S}^{0}$ SP4 signal Monte Carlo, and $(b)$ the $B^{0} \rightarrow$ $\phi K_{S}^{0}$ on-resonance sideband. 


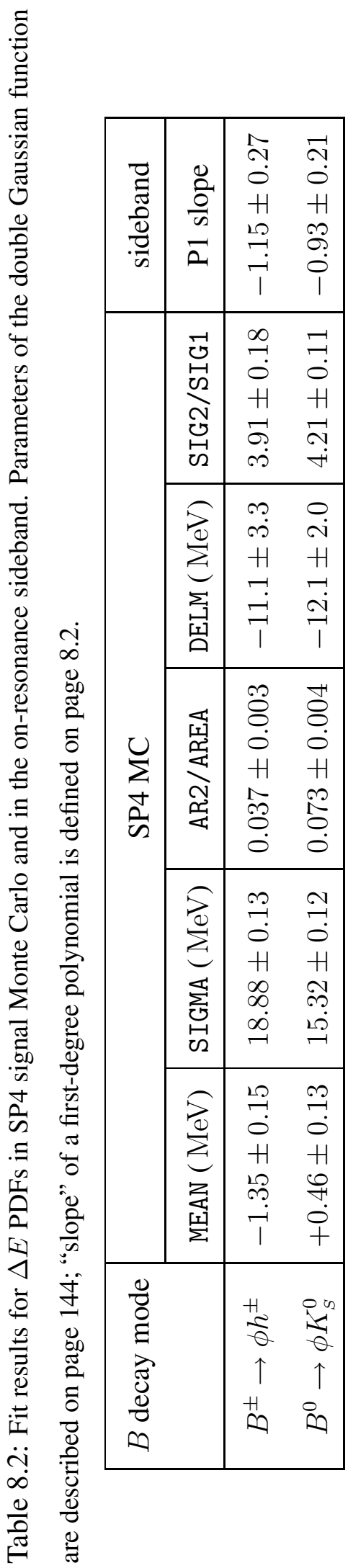


For a polynomial $a_{0}+a_{1}\left(x-x_{0}\right)$, the "slope" is defined as $a_{1} / a_{0}{ }^{1}$; in our case, $x_{0}=0$

\subsection{Fisher discriminant}

We fit Fisher discriminant distributions in signal Monte Carlo samples and the onresonance sideband with a bifurcated Gaussian (Fig. 8.6, Fig. 8.7, Table 8.3). As expected, mode-to-mode variations of $\mathcal{F}$ PDF parameters are statistically insignificant. The errors on parameters of signal Fisher PDFs used in systematics studies are obtained by comparing $\mathcal{F}$ distributions in SP4 Monte Carlo and data for the high-statistics calibration mode $B^{+} \rightarrow \pi^{+} \bar{D}^{0}\left(\bar{D}^{0} \rightarrow K^{+} \pi^{-}\right)$; errors on continuum background Fisher PDFs are obtained from on-resonance sideband data.

\subsection{Shape of the $\phi$ resonance}

The shape of the $\phi$ resonance used in SP4 Monte Carlo production is a non-relativistic Breit-Wigner with a cut-off at $m_{\phi_{\mathrm{PDG}}}-0.015=1.0044 \mathrm{GeV}$, the reason being the dependence of the $\phi$ shape on its decay mode. There are plans for SP5 to allow users to control the $\phi$ lineshape via a parameter in the user decay files for signal MC, when the $\phi$ decay mode is usually known; in the meantime, the existing lineshape is good enough

\footnotetext{
${ }^{1}$ Such choice of parametrization for a standard function is one of the many idiosyncrasies of MN_FIT [105], our preferred histogram-fitting package, that may seem odd at first but turn our to be quite convenient in practice.
} 

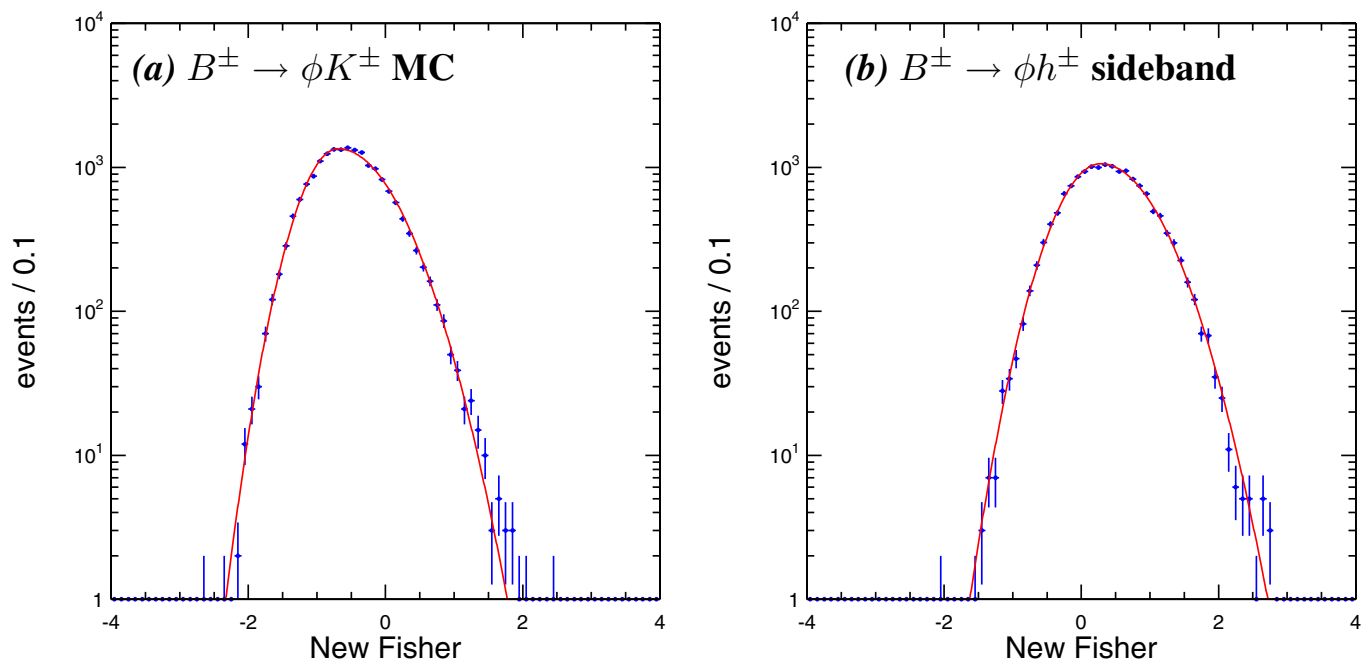

Figure 8.6: $\mathcal{F}$ distributions for $(a) B^{ \pm} \rightarrow \phi K^{ \pm}$signal Monte Carlo, and $(b)$ the $B^{ \pm} \rightarrow \phi h^{ \pm}$ on-resonance sideband.
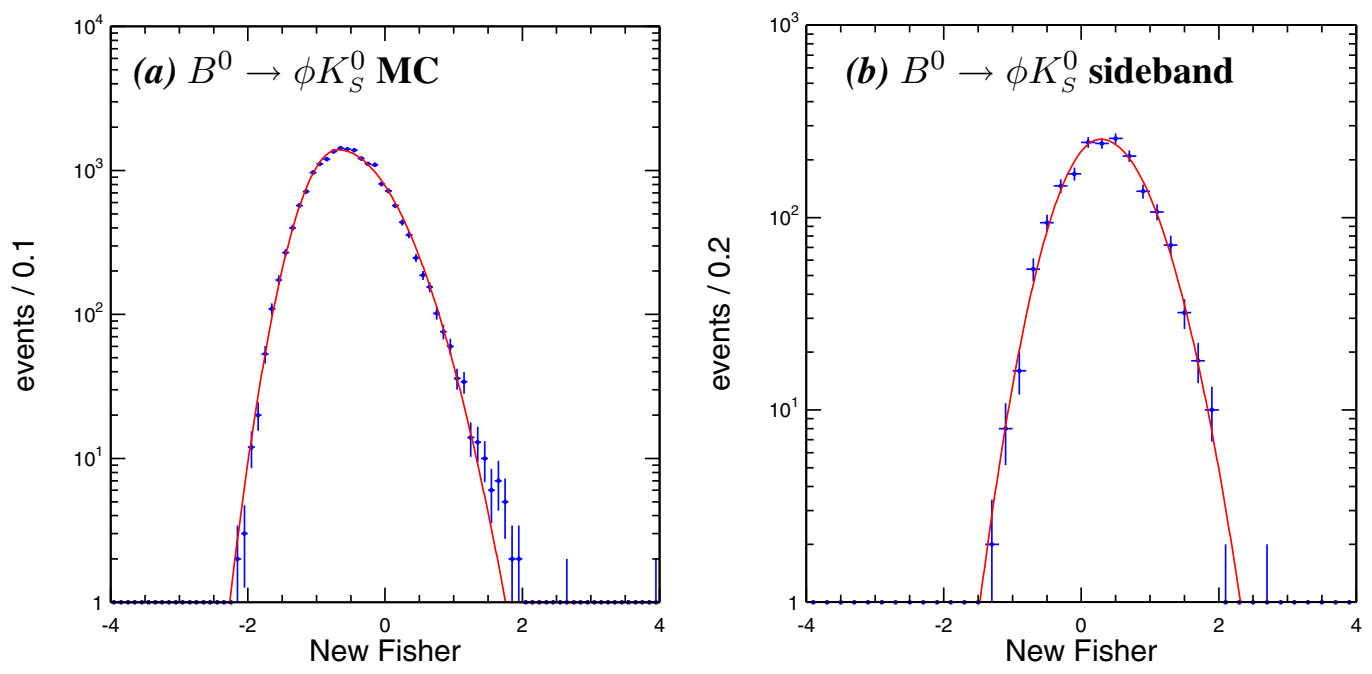

Figure 8.7: $\mathcal{F}$ distributions for $(a) B^{0} \rightarrow \phi K_{S}^{0}$ signal Monte Carlo, and $(b)$ the $B^{0} \rightarrow \phi K_{S}^{0}$ on-resonance sideband. 


\begin{tabular}{|c|c|c|c|}
\hline \multirow{3}{*}{ 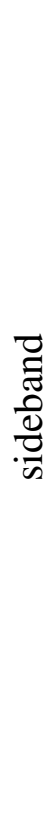 } & $6^{2}$ & $\begin{array}{l}0 \\
0 \\
0 \\
0 \\
0 \\
H \\
0 \\
0 \\
0 \\
0 \\
0\end{array}$ & $\begin{array}{l}\infty \\
\stackrel{0}{0} \\
0 \\
+ \\
H \\
ت \\
0 \\
0 \\
0\end{array}$ \\
\hline & $\vec{b}$ & 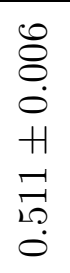 & $\begin{array}{l}\stackrel{N}{0} \\
0 \\
0 \\
+ \\
+ \\
\infty \\
\infty \\
\stackrel{1}{0} \\
0\end{array}$ \\
\hline & 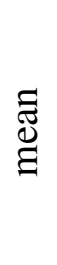 & 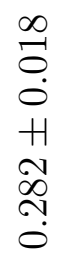 & 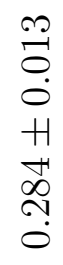 \\
\hline \multirow{3}{*}{$\begin{array}{l}\sum^{U} \\
\stackrel{ \pm}{\sim}\end{array}$} & $\sigma^{2}$ & $\begin{array}{l}\stackrel{0}{1} \\
0 \\
0 \\
0 \\
H \\
0 \\
0 \\
0 \\
0 \\
0\end{array}$ & 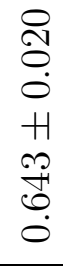 \\
\hline & $\vec{b}$ & 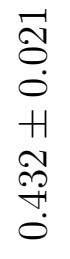 & $\begin{array}{l}\vec{\Omega} \\
0 \\
0 \\
+ \\
+ \\
\vec{J} \\
\vec{H}\end{array}$ \\
\hline & $\stackrel{\text { ‡ే }}{\stackrel{\Xi}{\Xi}}$ & $\begin{array}{l}0 \\
0 \\
0 \\
0 \\
H \\
H \\
-0 \\
0 \\
\dot{0} \\
1\end{array}$ & $\begin{array}{l}0 \\
0 \\
0 \\
0 \\
H \\
+1 \\
\sigma \\
0 \\
0 \\
0 \\
1\end{array}$ \\
\hline 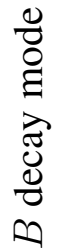 & & $\begin{array}{l}\stackrel{H}{a} \\
\uparrow \\
\stackrel{H}{\oplus}\end{array}$ & $\begin{array}{l}i=2 \\
\frac{1}{\theta} \\
\uparrow \\
0 \\
0\end{array}$ \\
\hline
\end{tabular}


to allow us to determine $B \rightarrow \phi K$ reconstruction efficiencies with a systematic error that is small compared to other sources of systematics.

The functional form of the $\phi$ resonance shape that we use is a convolution of a Gaussian resolution function with a relativistic spin-1 Breit-Wigner function with BlattWeisskopf damping factor correction (please refer to Appendix A.4 on page 145 for details). We take the radius of strong interaction $R=0.50 \pm 0.25$ fm from theory [63] and determine the width of the resolution Gaussian $\sigma_{\text {res }}=1.0 \pm 0.1 \mathrm{MeV}$ by fitting the core of the $m_{K K}$ distribution in SP4 Monte Carlo and assigning to it a conservative uncertainty.

The broad fake $\phi$ background that we fit with a second-degree polynomial can be classified into several categories. The first category are pairs of charged tracks one or both of which are not kaons. These constitute about a third of the fake $\phi$ 's and can be eliminated by tightening the $\phi \rightarrow K^{+} K^{-}$PID cuts. We verified that doing so would noticeably harm signal reconstruction efficiency and decrease sensitivity of the analysis. The remaining $\sim 2 / 3$ of the fake $\phi$ candidates are pairs of oppositely-charged kaons, which may be random combinations of tracks, products of non-resonant decays of $B$ or $D$ mesons, or decay products of other resonances.

A second-degree polynomial should provide an adequate description of the first two sources of $K^{+} K^{-}$pairs (we checked and found higher-order terms not to be statistically significant); however, the contribution from tails of other resonances will only be adequately accounted for in a polynomial description of the fake $\phi$ shape if the resonances in question are sufficiently distant and wide compared with the width of the $\phi$ 
Table 8.4: Parameters of the shape of the fake $\phi$ background and the fraction of real $\phi$ 's in the on-resonance sideband. The fake $\phi$ shape is fitted with a second-degree polynomial of the form $a_{0}+a_{1}\left(x-m_{\phi_{\mathrm{PDG}}}\right)+a_{2}\left(x-m_{\phi_{\mathrm{PDG}}}\right)^{2}$, where $m_{\phi_{\mathrm{PDG}}}=1.0194 \mathrm{GeV}$.

\begin{tabular}{|c|c|c|c|}
\hline$B$ decay mode & $a_{1} / a_{0}$ & $a_{2} / a_{0}$ & Fraction of real $\phi ' s$ \\
\hline$B^{ \pm} \rightarrow \phi h^{ \pm}$ & $9.44 \pm 0.71$ & $-250 \pm 41$ & $0.325 \pm 0.042$ \\
$B^{0} \rightarrow \phi K_{S}^{0}$ & $11.7 \pm 1.8$ & $-218 \pm 130$ & $0.407 \pm 0.023$ \\
\hline
\end{tabular}

mass window. The projection plots of the $K^{+} K^{-}$invariant mass on page 116 extend to $1.2 \mathrm{GeV}$, well beyond the $\phi$ mass window, and seem to indicate quite conclusively that higher resonances decaying into $K^{+} K^{-}$do not jeopardize the validity of our fake $\phi$ parametrization.

Table 8.4 lists parameters of the fake $\phi$ background PDFs and fractions of real $\phi$ 's in the on-resonance sideband.

\section{5 $\phi$ helicity}

Both $B^{ \pm} \rightarrow \phi h^{ \pm}$and $B^{0} \rightarrow \phi K_{S}^{0}$ are decays of a pseudoscalar particle into a pseudoscalar and a vector, so polarization of the vector meson $\phi$ in this decay is known, and the $\mathcal{H}$ PDF is a parabola (Fig. 8.9a, Fig. 8.10a). One would naively expect the fake $\phi$ 's in the continuum background to be nearly unpolarized (which corresponds to a flat $\mathcal{H}$ distribution), and the real $\phi$ 's to be only partially polarized. While the second part of the previous statement is certainly true, the first one is not, one part of the reason being angular and flavor correlations among products of primary quark hadronization 


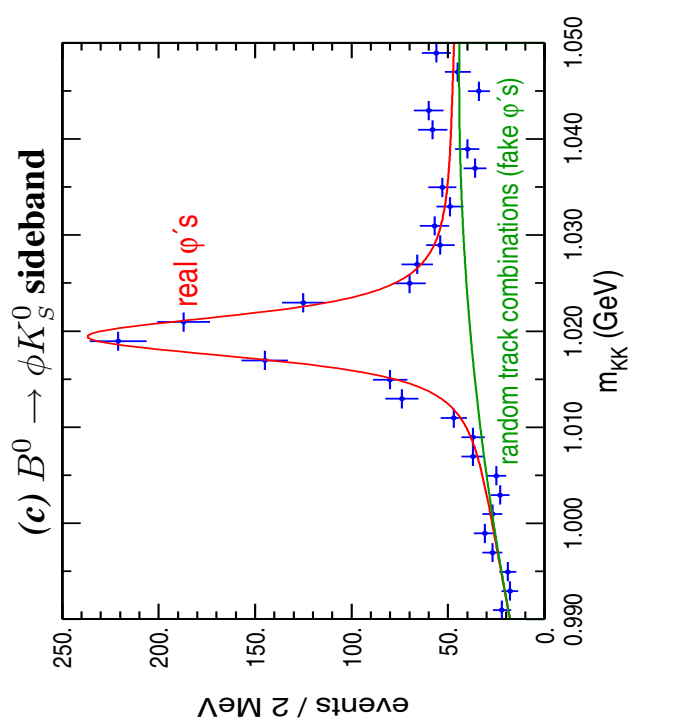

0
0
1
0
0
0
0
0
0
0
0
0
0
0
0
0
0
0
0
0
0
0
0
0
0
0

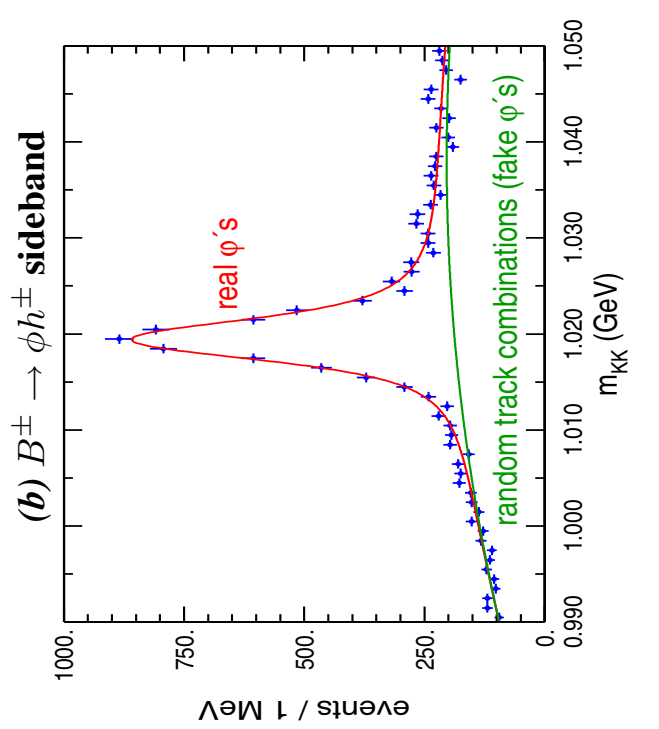

$\uparrow$

in

2

ำ

䒕

$\Sigma$

코ำ

क

范

$\frac{1}{a}$

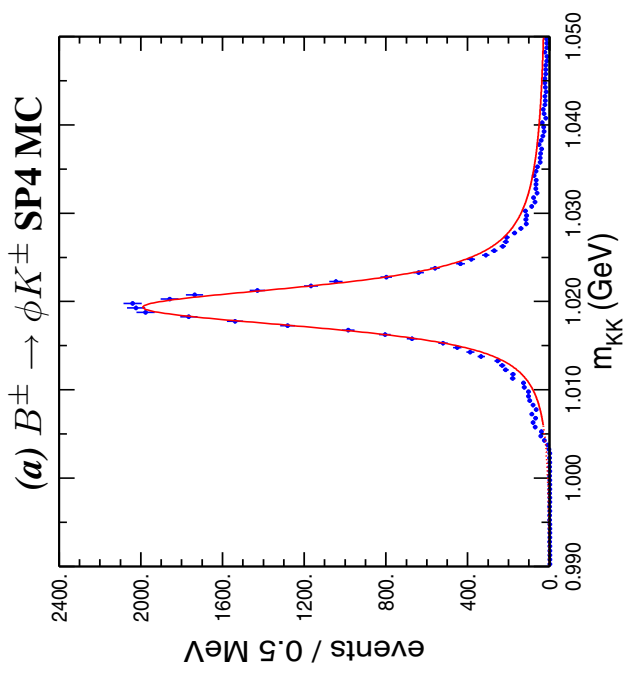

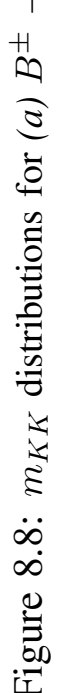

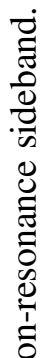


Table 8.5: Fit results for $\mathcal{H}$ distributions for real and fake $\phi$ 's in the on-resonance sideband.

\begin{tabular}{|c|c|c|}
\hline$B$ decay mode & $b_{2} / b_{0}$, fake $\phi$ 's & $b_{2} / b_{0}$, real $\phi$ 's \\
\hline$B^{ \pm} \rightarrow \phi h^{ \pm}$ & $0.247 \pm 0.066$ & $0.555 \pm 0.134$ \\
$B^{0} \rightarrow \phi K_{S}^{0}$ & $0.660 \pm 0.166$ & $0.147 \pm 0.130$ \\
\hline
\end{tabular}

and fragmentation, and another part being existence of other, much broader resonance states decaying into a pair of charged tracks both of which are either kaons or pions misidentified as kaons.

We employ a five-step iterative procedure that makes use of the known shapes of the $\phi$ resonance, the fake $\phi$ background, and their relative fractions in the on-resonance sideband to extract polarizations of the real and the fake $\phi$ components of the continuum background. Histograms representative of the procedure are shown in Fig. 8.9b-d and Fig. 8.10b-d; Table 8.5 summarizes results of fitting the final set of $\mathcal{H}$ histograms with a second-degree polynomial with no linear term, $b_{0}+b_{2} x^{2}$. 


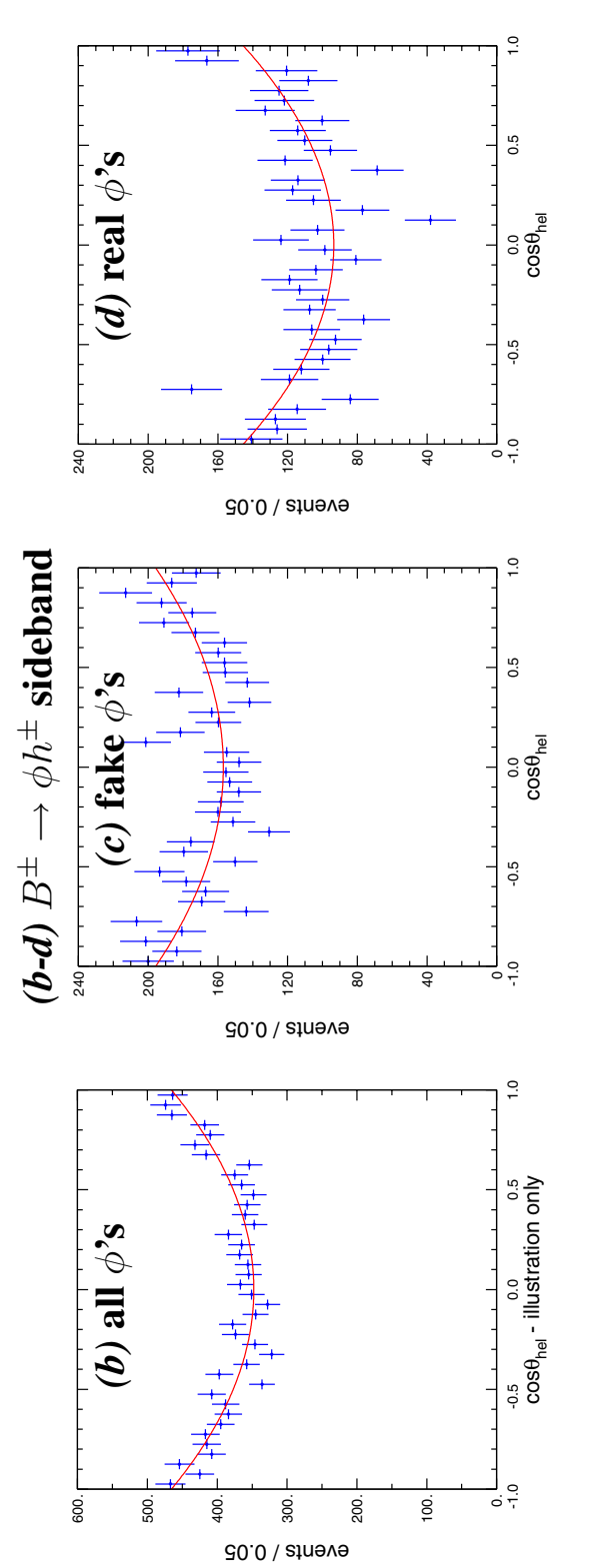

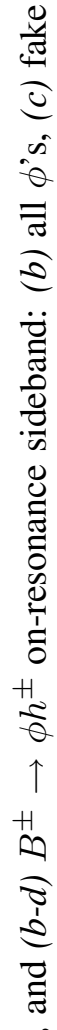

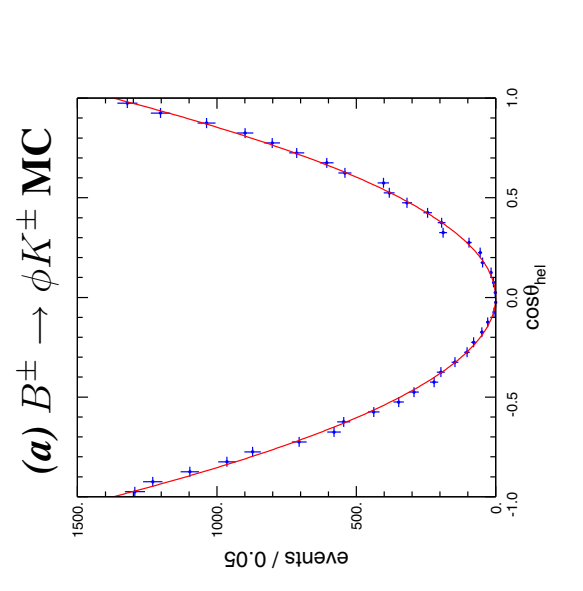

Uี

气ั

స్

$+$

$\uparrow$ हู

in

(2)

કั)

ฮี

音

ت

$\tau$ is

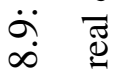

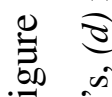

i 


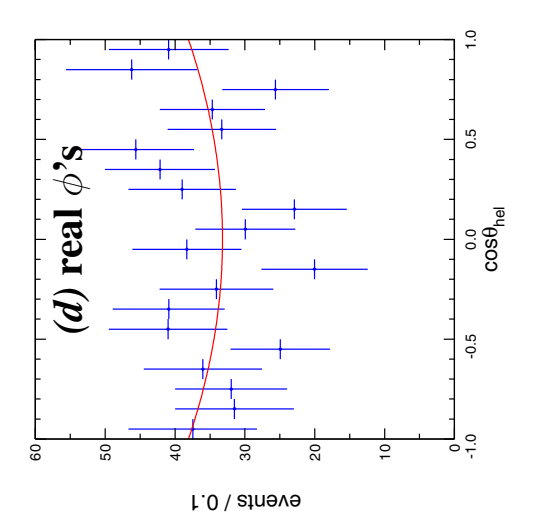

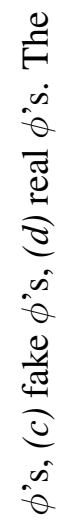

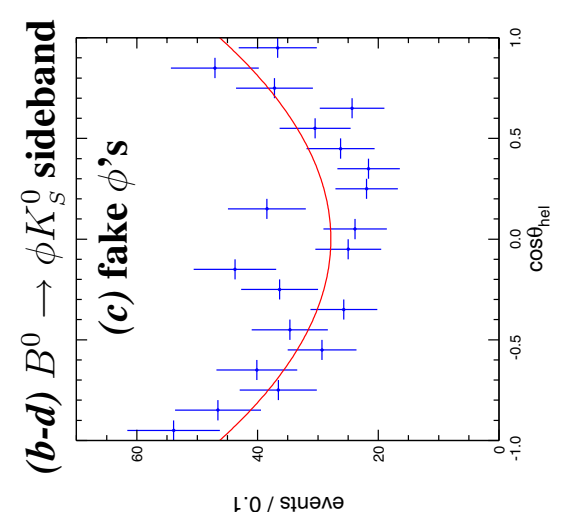

ส

2

تृ

음

先

$\uparrow$

ริำ

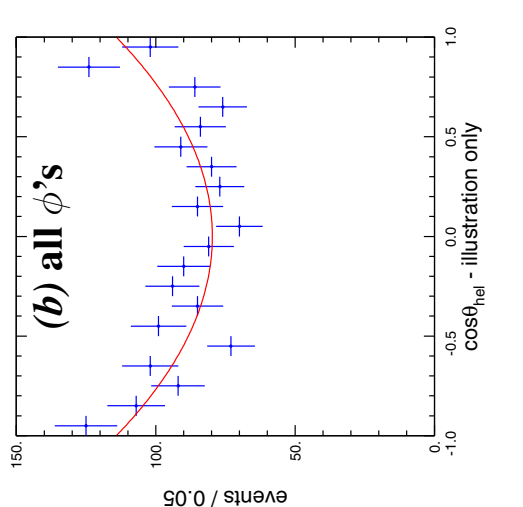

్ㅗ

กั

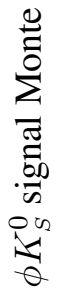

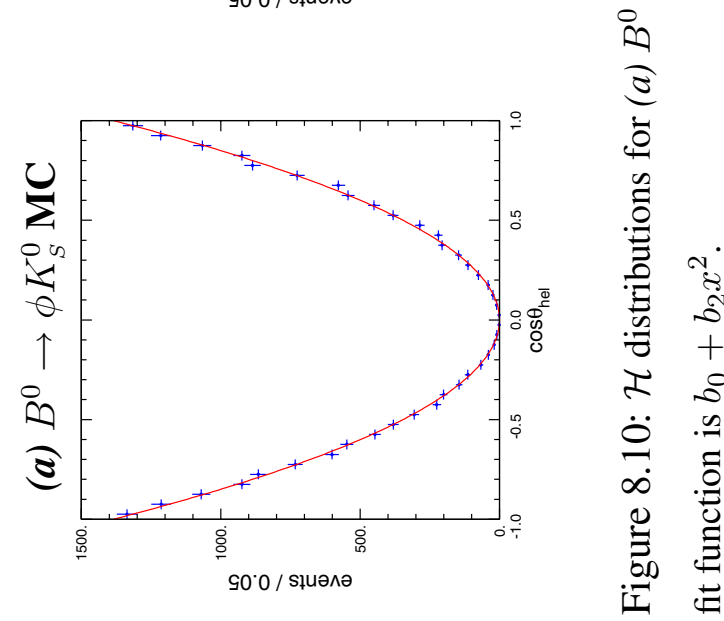




\section{Chapter 9}

\section{Efficiency and Production Rate}

\subsection{Reconstruction and ML fit efficiencies}

The reconstruction efficiency, i.e., the fraction of originally generated signal Monte Carlo events that pass all cuts and are input into the ML fitter, is determined for each of the three modes using large samples of SP4 MC events (see Table 9.1). The statistical errors on the values of reconstruction efficiencies are negligibly small and we do not keep them.

We determine the ML fit efficiency for each of the three decay modes by running the ML fitter on samples of signal Monte Carlo optionally mixed with a varied number of 'toy' simulated background events randomly generated according to the continuum PDFs. The values of ML fit efficiencies that we use in the conversion of fit event yields into branching fractions are taken from performing the fit on pure signal MC; the values obtained in presence of toy background events are used in determination of the system- 


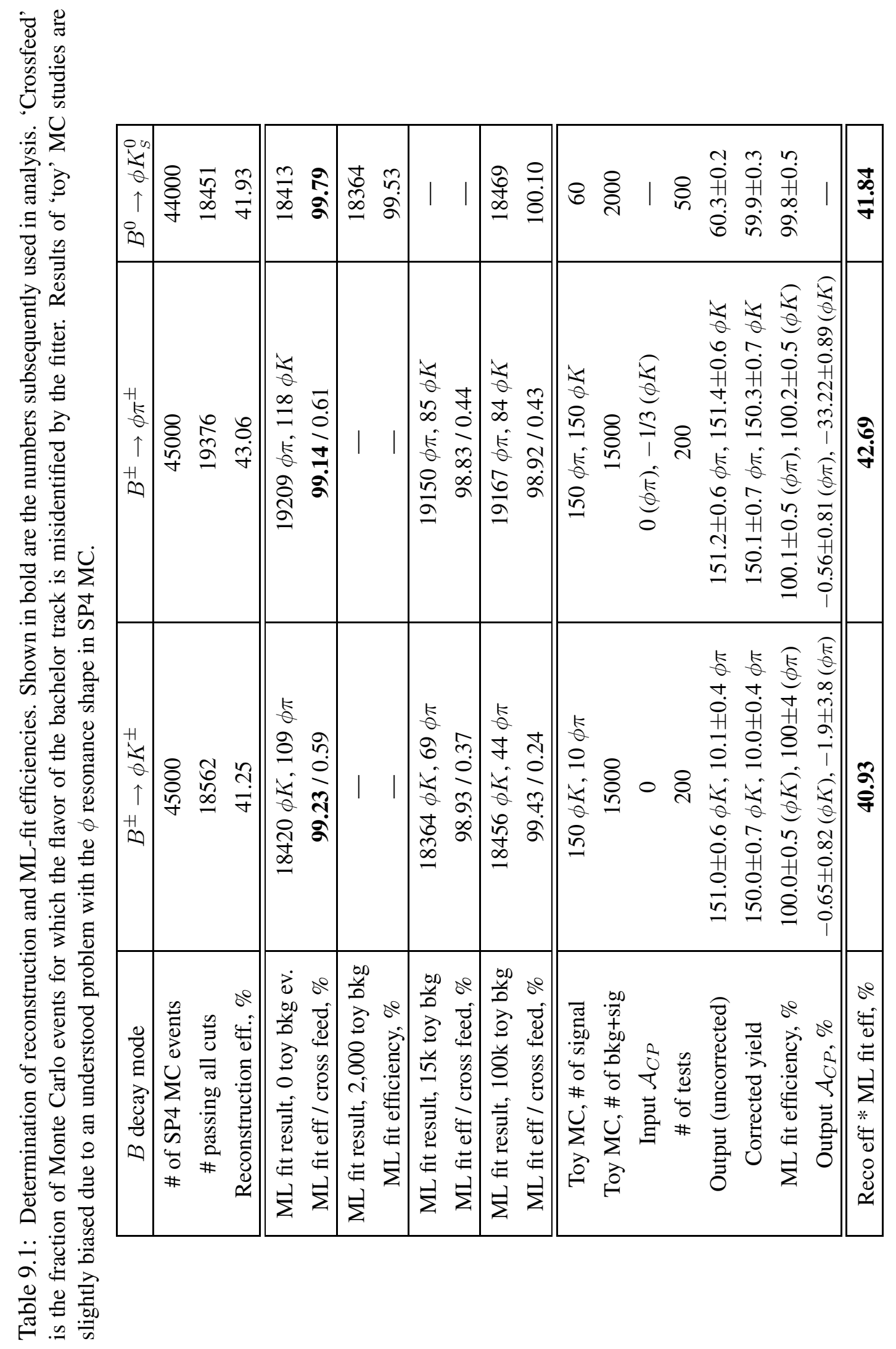


atic errors.

We have also conducted several sets of 'toy' Monte Carlo studies in which background events are generated according to the provided PDFs while signal events are pulled at random from SP4 signal Monte Carlo; in these studies, the numbers of both background and signal events were chosen to be close to the numbers in the actual fits to data. Special attention has been devoted to proper simulation of the correlation between the $\Delta E$ and $\theta_{\text {Cherenkov }}$ separations between the $B^{ \pm} \rightarrow \phi K^{ \pm}$and $B^{ \pm} \rightarrow \phi \pi^{ \pm}$hypotheses that is unique to the environment of an asymmetric $B$-meson factory (for an in-depth discussion of $\pi^{ \pm} / K^{ \pm}$separation, see Section 11.2 on page 115$)$.

Unfortunately, our toy MC studies suffer from the imprecise $\phi$ resonance shape used in SP4 MC: the positive-side tail of the $\phi$ mass distribution in SP4 MC is significantly underestimated, which makes a number of background events be 'pulled' into the signal category to fill the empty tail of the $P$-wave Breit-Wigner shape programmed into the fitter. Excluding $m_{K K}$ from the list of ML fit variables eliminates the $\sim 0.7 \%$ bias at the price of a $\sim 20 \%$ increase of the fit's error.

Note that the cross feed of the decays $B^{ \pm} \rightarrow \phi K^{ \pm}$and $B^{ \pm} \rightarrow \phi \pi^{ \pm}$into each other is well below $1 \%$. The fact that it goes down in the presence of 'toy' background events is easy to understand: for instance, the $B^{ \pm} \rightarrow \phi K^{ \pm}$events that are misidentified by the fitter have a probability distribution in the multi-dimensional space of ML fit parameters that looks neither like the probability distribution for $B^{ \pm} \rightarrow \phi \pi^{ \pm}$nor like the probability distribution for background. As more and more background events are added, more and more of the misidentified $B^{ \pm} \rightarrow \phi K^{ \pm}$events 'dissolve' into what looks to the fitter as 
fluctuations of the background. With the increasing number of background events, the fraction of $B^{ \pm} \rightarrow \phi K^{ \pm}$events misidentified as $B^{ \pm} \rightarrow \phi \pi^{ \pm}$asymptotically approaches a constant, which in this case is not only non-zero, but is quite large-about $70 \%$ of the cross feed rate in the absence of background-because the shape and the kinematics of the two decays are very similar. In a more general case-for example, for cross feed from $B \rightarrow \phi K^{*}$ decays into $B \rightarrow \phi h$, as will be shown in Section $11-$ a cross feed prominent in the absence of continuum background may completely dissolve into it once even a relatively small number of 'toy' background events are added to signal MC.

All methods of determining the ML fit efficiency are in good agreement with each other. We use $1.0 \%$ as a reasonably conservative estimate of the systematic error.

\subsection{Tracking efficiency}

To obtain the total efficiency, the 'raw' MC efficiency has to be corrected for the known discrepancies between tracking efficiencies in SP4 Monte Carlo and data. We follow the recommendations and prescriptions of the Tracking Efficiency Task Force given in [88]. We compute the averages over the $1900 \mathrm{~V}, 1930 \mathrm{~V}$ and $1960 \mathrm{~V}$ DCH voltage data sets of the product of data/MC tracking efficiency ratios for all GoodTracksLoose among the $B$ meson decay products (i.e., the kaons in $\phi \rightarrow K^{+} K^{-}$and the bachelor tracks in $B^{ \pm} \rightarrow \phi h^{ \pm}$-pions in $K_{S}^{0} \rightarrow \pi^{+} \pi^{-}$come from the ChargedTracks list and require no correction) and then weigh these averages according to the number of $B \bar{B}$ pairs in 
each of the three data sets. The resulting efficiency correction factors are 0.9727 for $B^{ \pm} \rightarrow \phi K^{ \pm}, 0.9725$ for $B^{ \pm} \rightarrow \phi \pi^{ \pm}$, and 0.9809 for $B^{0} \rightarrow \phi K_{S}^{0}$ (the statistical errors are negligible).

Following the recommendations in [88], the systematic error on the tracking efficiency correction is $0.8 \%$ per GoodTracksLoose track and $1.3 \%$ per ChargedTracks track, i.e., $2.4 \%$ in $B^{ \pm} \rightarrow \phi h^{ \pm}$and $4.2 \%$ in $B^{0} \rightarrow \phi K_{S}^{0}$.

\section{3 $K_{S}^{0}$ reconstruction efficiency in $B^{0} \rightarrow \phi K_{S}^{0}$}

A large semi-inclusive sample of $K_{S}^{0}$ candidates, 1.4 million in data and 2.0 million in MC, is used to study the relative $K_{S}^{0}$ reconstruction efficiencies in Release-10 data and SP4 Monte Carlo (see Appendix C). We subdivide the samples into a large number of bins in momentum, polar angle and transverse $(X Y) K_{S}^{0}$ flight length; bin-by-bin ratios normalized to the $[0.3 \mathrm{~cm}, 1.3 \mathrm{~cm}]$ bin in the $X Y K_{S}^{0}$ flight length and their respective statistical errors are then computed; the difference between the $K_{S}^{0}$ mass resolutions in data and $\mathrm{MC}$ is also taken into account. The resulting $K_{S}^{0}$ reconstruction efficiency correction tables are applied to signal Monte Carlo for the modes $B^{0} \rightarrow \phi K_{S}^{0}$ and $B^{0} \rightarrow \pi^{+} D^{-}\left(D^{-} \rightarrow K_{S}^{0} \pi^{-}\right)$, which provide $K_{S}^{0}$ spectra representative of most cases of $K_{S}^{0}$ use in $B A B A R$ analyses, to determine the overall correction factor and its statistical error. The above exercise is repeated for several sets of $K_{S}^{0}$ quality cuts, from none to tight, and for three different binning approaches in order to determine the systematic error. 
We determine the $K_{S}^{0}$ reconstruction efficiency correction factor to be equal to $0.967 \pm$ 0.020 , where the quoted systematic error does not include the $\pm 2.6 \%$ tracking efficiency uncertainty mentioned in the previous subsection.

Note that although the pions used in $K_{S}^{0} \rightarrow \pi^{+} \pi^{-}$reconstruction are taken from the ChargedTracks list, the imposition of $K_{S}^{0}$ quality cuts (on the $K_{S}^{0} \rightarrow \pi^{+} \pi^{-}$invariant mass, flight length significance and flight direction, see page 67) may impose a requirement for reasonably good kinematic resolution on the pion tracks, i.e., for presence of DCH hits. The following procedure allows us to determine the systematic error for this effect:

error $=\left(1-\varepsilon_{\mathrm{GTL}}\right) \times\left(1-\frac{B 2 / B 1}{A 2 / A 1}\right)=(1-0.9835) \times\left(1-\frac{271 / 334}{22855 / 23792}\right)=0.0026$,

where $\varepsilon_{\mathrm{GTL}}$ is the value of the tracking efficiency correction on the $K_{S}^{0}$ decay products computed as if they were required to pass the selection criteria of the GoodTracksLoose list; $A 1$ and $B 1$ are numbers of $K_{S}^{0} \rightarrow \pi^{+} \pi^{-}$candidates in signal $B^{0} \rightarrow \phi K_{S}^{0} \mathrm{MC}$ that, respectively, pass the GoodTracksLoose criteria for both tracks or fail for one or both tracks; $A 2$ and $B 2$ are the numbers of such candidates that also pass the $K_{S}^{0}$ quality cuts. Thus, this source of systematic error is negligibly small.

\subsection{Particle identification efficiency}

We use a standard PID selector, PidKaonSMSSelector in its notApion mode, to identify kaons in the decay $\phi \rightarrow K^{+} K^{-}$. The PID tools group provides "PID killing" tables 
([103]) that list charge-dependent PID efficiencies in data for all particle types and all officially supported PID selectors in bins of angle and momentum. Separate tables exist for each of the three years of BABAR operation $(2000,2001,2002)$. Although the existing tables were all created only for GoodTracksVeryLoose tracks, we believe that they can be directly applied to our analysis because significant differences between GTL and GTVL tracks in PID selector performance exist only at very small track momenta, which are not available in $B \rightarrow \phi h$ (see Figure 9.1a). Indeed, fewer of $7 \%$ of GTVL tracks in $B A B A R$ that have momenta above $600 \mathrm{MeV}$ in the laboratory frame are not also GTL tracks (see Figure 9.1b). Furthermore, for the vast majority of $\phi \rightarrow K^{+} K^{-}$tracks the bulk of PID information comes from the DIRC and is therefore insensitive to the tracking quality cuts that distinguish the GTVL and the GTL lists (page 57).

So, of the $\sim 7 \%$ of GTVL tracks that are not also GTL tracks, $\lesssim 15 \%$ have their PID information dominated by $\mathrm{d} E / \mathrm{d} x$. Suppose, very pessimistically, that there is a 10\% discrepancy between PidKaonSMSSelector (notApion) efficiencies in SP4 MC and data for such tracks. Multiplying the three numbers, we obtain a negligible upper limit of $0.1 \%$ on the systematic error due to the possible discrepancies between the PID efficiency tables made for GTVL and GTL tracks. The systematic due to the fact that the official PID efficiency tables do not take into account our choice of proton and electron vetoes is also very small, since of all MC $B^{ \pm} \rightarrow \phi K^{ \pm}$signal events only a handful have $\phi \rightarrow K^{+} K^{-}$tracks that fail either of the two vetoes, and any efficiency correction on that handful of events would be too minuscule to worry about.

We determine that the efficiencies of applying PidKaonSMSSelector (notApion) to 


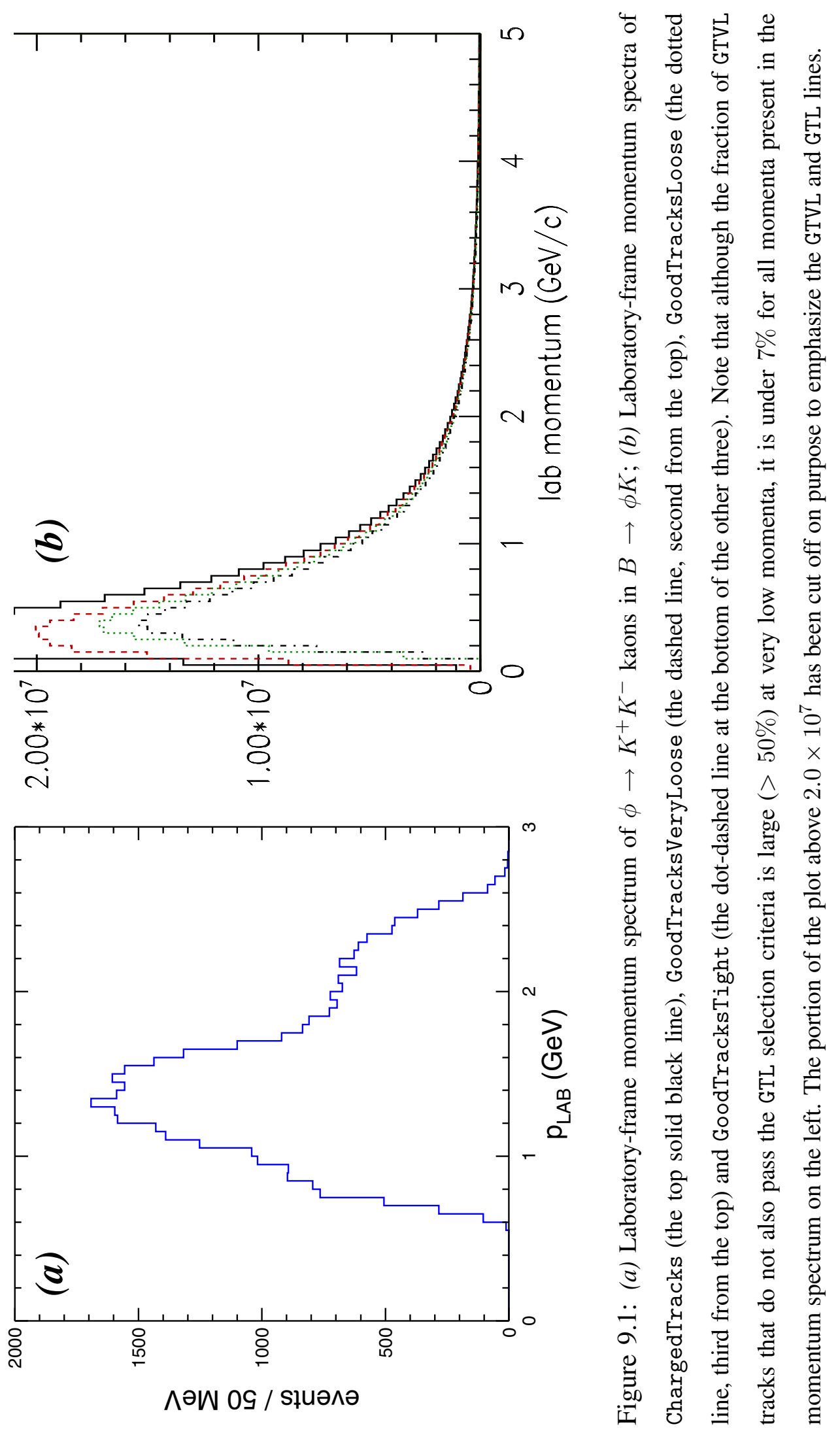


GTL $\phi \rightarrow K^{+} K^{-}$tracks in SP4 MC is remarkably consistent with efficiency of the same selector applied to GTVL tracks with the same momentum spectrum in data: we compare the PID selector efficiencies in SP4 Monte Carlo with values in the "PID killing" tables, weigh them according to the numbers of $B \bar{B}$ pairs in each of the three time periods, and find the following data/MC PID efficiency correction factors: 1.0000 for $B^{ \pm} \rightarrow \phi K^{ \pm}$, 0.9981 for $B^{ \pm} \rightarrow \phi \pi^{ \pm}$, and 0.9962 for $B^{0} \rightarrow \phi K_{S}^{0}$.

Since official recommendations for assigning systematic errors to these values do not yet exist, we are choosing to use a rather conservative value of $1.0 \%$ per $K^{ \pm}$.

\section{$9.5 \Upsilon(4 S)$ and daughter branching fractions}

In the interpretation of our results we assume that the production rates of charged and neutral $B$-meson pairs at the peak of the $\Upsilon(4 S)$ resonance are equal:

$$
R_{\Upsilon(4 S)}^{+/ 0}=\frac{\Gamma\left(\Upsilon(4 S) \rightarrow B^{+} B^{-}\right)}{\Gamma\left(\Upsilon(4 S) \rightarrow B^{0} \bar{B}^{0}\right)}=1
$$

This agrees with current measurements $[74,75]$ and theoretical predictions [68] and is consistent with practices and recommendations of the Particle Data Group [10].

Finally, we correct the efficiencies for the branching fractions of unstable particles in the $B$ decay trees, in our case the $\phi$ and the $K_{S}^{0}$. The relevant branching fractions are $\mathcal{B}\left(\phi \rightarrow K^{+} K^{-}\right)=0.492 \pm 0.007$ and $\mathcal{B}\left(K_{S}^{0} \rightarrow \pi^{+} \pi^{-}\right)=0.6860 \pm 0.0027$ [8], as well as $K_{S}^{0}=\frac{1}{2} K^{0}$. 


\section{Chapter 10}

\section{Physics Results}

Results of maximum-likelihood fits to on-resonance data are given in Table 10.1 for $B^{ \pm} \rightarrow \phi K^{ \pm}$and $B^{ \pm} \rightarrow \phi \pi^{ \pm}$and in Table 10.2 for $B^{0} \rightarrow \phi K_{S}^{0}$. The errors on the branching fractions and direct $C P$ asymmetries shown in these tables are statistical only. The correlation matrices of the ML input variables can be found in Appendix B. 
Table 10.1: Maximum-likelihood fit results for the modes $B^{ \pm} \rightarrow \phi K^{ \pm}$and $B^{ \pm} \rightarrow \phi \pi^{ \pm}$(errors are statistical only).

\begin{tabular}{|c|c|c|c|}
\hline data set & all data & 1999-2001 & 2002 \\
\hline$\#$ of $B \bar{B}$ pairs, $10^{6}$ & $88.9 \pm 1.0$ & $61.5 \pm 0.7$ & $27.4 \pm 0.3$ \\
\hline Events to fit & 14371 & 9835 & 4536 \\
\hline Signal yield, $\phi K$ & $173.4_{-14.5}^{+15.3}$ & $114.8_{-11.8}^{+12.6}$ & $58.5_{-8.3}^{+9.0}$ \\
\hline Signal $\mathcal{A}_{C P}, \phi K$ & $(+3.9 \pm 8.6) \%$ & $(+2.9 \pm 10.6) \%$ & $(+5.6 \pm 14.8) \%$ \\
\hline Signal yield, $\phi \pi$ & $0.9_{-0.9}^{+2.8}$ & $0.0_{-0.0}^{+3.7}$ & $0.8_{-0.8}^{+2.5}$ \\
\hline Signal yield, $\phi \pi, 90 \% \mathrm{UL}$ & $<6.5$ & $<5.6$ & $<6.4$ \\
\hline $\operatorname{Bkg} K^{ \pm}$fraction & $0.379 \pm 0.004$ & $0.379 \pm 0.005$ & $0.381 \pm 0.007$ \\
\hline $\operatorname{Bkg} \mathcal{A}_{C P}, K$ & $(+0.6 \pm 1.4) \%$ & $(+1.3 \pm 1.7) \%$ & $(-1.0 \pm 2.5) \%$ \\
\hline $\operatorname{Bkg} \mathcal{A}_{C P}, \pi$ & $(-0.8 \pm 1.1) \%$ & $(-1.3 \pm 1.3) \%$ & $(+0.3 \pm 1.9) \%$ \\
\hline Combinations/events & \multicolumn{3}{|c|}{1.002} \\
\hline MC raw $\varepsilon(\%)$ & \multicolumn{3}{|c|}{40.93 for $\phi K$} \\
\hline Tracking $\varepsilon$ corr. & \multicolumn{3}{|c|}{0.9727 for $\phi K$} \\
\hline PID $\varepsilon$ corr. & \multicolumn{3}{|c|}{1.0000 for $\phi K$} \\
\hline$\prod \mathcal{B}_{i}$ & \multicolumn{3}{|c|}{0.492} \\
\hline Total $\varepsilon(\%)$ & \multicolumn{3}{|c|}{19.59 for $\phi K$} \\
\hline $\mathcal{B}(\phi K), 10^{-6}$ & $9.96_{-0.84}^{+0.88}$ & $9.52_{-0.98}^{+1.04}$ & $10.92_{-1.54}^{+1.69}$ \\
\hline Significance (stat.) $(\sigma)$ & 25.6 & 20.4 & 15.4 \\
\hline $\mathcal{B}(\phi \pi), 10^{-6}$ & $<0.37$ & $<0.45$ & $<0.115$ \\
\hline Significance (stat.) $(\sigma)$ & 0.46 & 0.04 & 0.59 \\
\hline
\end{tabular}


Table 10.2: Maximum-likelihood fit results for the mode $B^{0} \rightarrow \phi K_{S}^{0}$ (errors are statistical only). Note that the final result is expressed as a branching fraction for the final state $\phi K^{0}$, not $\phi K_{S}^{0}$.

\begin{tabular}{|c|c|c|c|}
\hline data set & all data & 1999-2001 & 2002 \\
\hline$\#$ of $B \bar{B}$ pairs, $10^{6}$ & $88.9 \pm 1.0$ & $61.5 \pm 0.7$ & $27.4 \pm 0.3$ \\
\hline Events to fit & 2043 & 1377 & 666 \\
\hline Signal yield, $\phi K_{S}^{0}$ & $49.8_{-7.9}^{+8.7}$ & $36.8_{-6.8}^{+7.6}$ & $13.4_{-3.8}^{+4.6}$ \\
\hline Combinations/events & \multicolumn{3}{|c|}{1.002} \\
\hline $\mathrm{MC}$ raw $\varepsilon(\%)$ & \multicolumn{3}{|c|}{41.84} \\
\hline Tracking $\varepsilon$ corr. & \multicolumn{3}{|c|}{0.9809} \\
\hline$K_{S}^{0} \varepsilon$ corr. & \multicolumn{3}{|c|}{0.967} \\
\hline PID $\varepsilon$ corr. & \multicolumn{3}{|c|}{0.9962} \\
\hline$\prod \mathcal{B}_{i}$ & \multicolumn{3}{|c|}{0.1688} \\
\hline Total $\varepsilon(\%)$ & \multicolumn{3}{|c|}{6.67} \\
\hline $\mathcal{B}\left(\phi K^{0}\right), 10^{-6}$ & $8.4_{-1.3}^{+1.5}$ & $8.9_{-1.7}^{+1.9}$ & $7.3_{-2.1}^{+2.5}$ \\
\hline Significance (stat.) $(\sigma)$ & 12.7 & 10.5 & 7.1 \\
\hline
\end{tabular}




\section{Chapter 11}

\section{Cross Checks}

\subsection{Projection plots}

Perhaps the best way to conclusively convince oneself and others of the sanity of a maximum-likelihood analysis is to produce plots showing likelihood projections onto the input variables overlayed with curves showing the amounts of background and of the total number of events expected for the efficiency and branching fraction values obtained in the course of the analysis.

Here we present plots made with the full Summer 2002 data set after a cut on probability ratios chosen to emphasize the signal and computed with the plotted variable being excluded from the fit: we require

$$
\mathcal{P}_{\text {sig }} /\left(\mathcal{P}_{\text {sig }}+\mathcal{P}_{\text {bkg }}\right) \geq 0.85
$$

and, additionally for $B^{ \pm} \rightarrow \phi h^{ \pm}\left(h^{ \pm}=\pi^{ \pm}, K^{ \pm}\right)$, 


$$
\mathcal{P}_{\mathrm{h}^{ \pm} \text {sig }} /\left(\mathcal{P}_{\mathrm{K}^{ \pm} \text {sig }}+\mathcal{P}_{\pi^{ \pm} \text {sig }}\right) \geq 0.51 .
$$

The projection plots are made for all ML variables: $m_{\mathrm{ES}}, \Delta E, m_{K K}, \mathcal{H}, \mathcal{F}$, and the Cherenkov angle pull (Fig. 11.1, Fig. 11.2, Fig. 11.3, Fig. 11.7, Fig. 11.4, Fig. 11.8, Fig. 11.5). The dashed curves indicate the expected continuum background contributions.

Please note once again that the curves in projection plots are not fits to the histograms. Projections plots made with an arbitraty cut on the signal/background probability ratio to emphasize likely signal events over background. We plot a histogram of the events that pass that cut, overlaid with curves that indicate the amount and shape of signal and background based on the output of the ML fit, the PDF shapes and the efficiency of the probability-ratio cut that is determined from Monte Carlo. These projection plots are intended only as an illustration of the maximum-likelihood fit performance; one cannot say that "the curve does not match the data well enough" because only a fraction of the data are plotted, and the number of events in the histogram is thus subject to statistical fluctuations. The looser the signal-emphasizing probabilityratio cut, the lower the likelihood that the agreement between the histogram and and curve will be visually displeasing. This is demonstrated in Fig. 11.6, which contains a different version of $m_{\mathrm{ES}}$ and $\Delta E$ projection plots for the modes $B^{ \pm} \rightarrow \phi K^{ \pm}$and $B^{0} \rightarrow \phi K_{S}^{0}$. The difference from the previously shown projection plots is that here $\mathcal{P}_{\text {sig }} /\left(\mathcal{P}_{\text {sig }}+\mathcal{P}_{\text {bkg }}\right) \gtrsim 0.5$ instead of $\geq 0.85$, as in Eq. 11.1. 


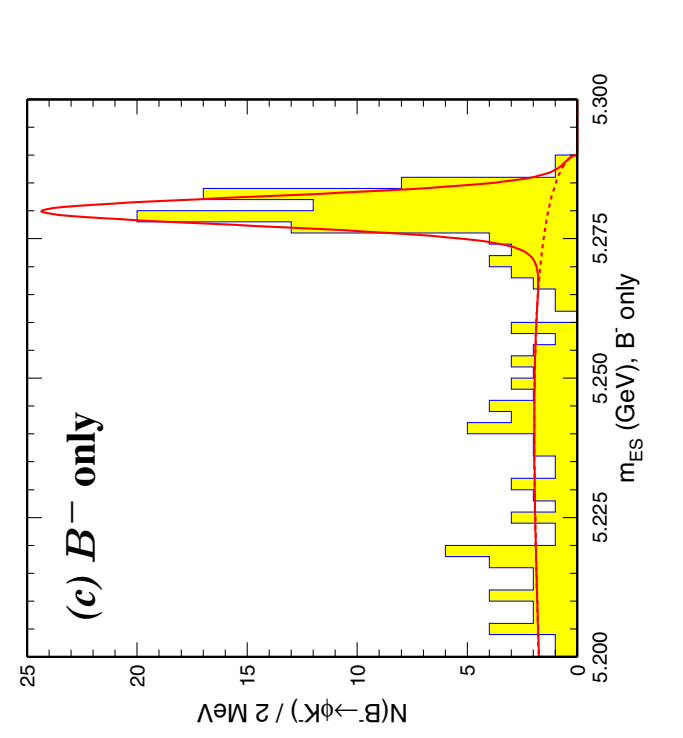

츰

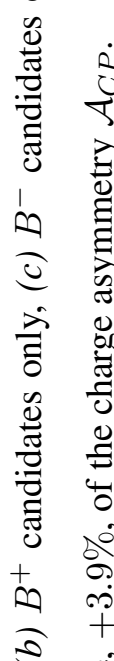

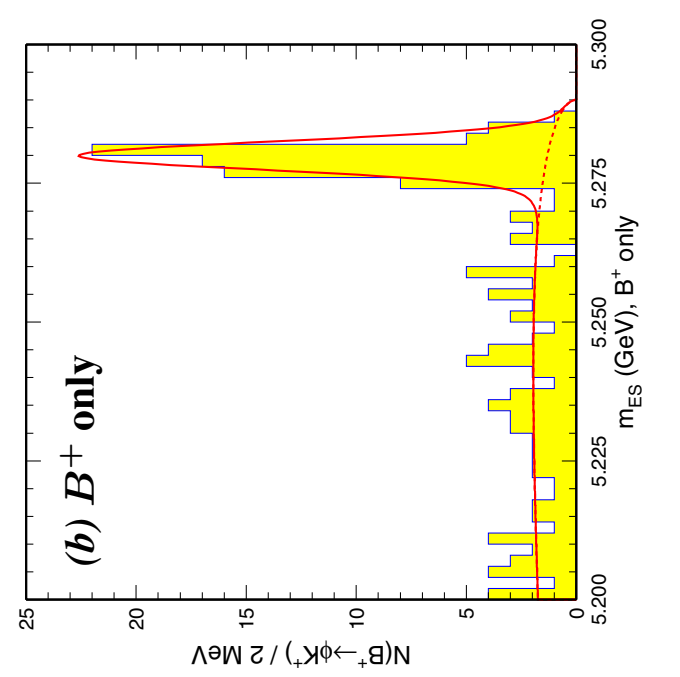

兽

H

$\bar{\pi}$

छิ

$\ddot{H} \equiv$

$\frac{1}{8}$

$\uparrow \stackrel{0}{0}$

in

ฮ

留

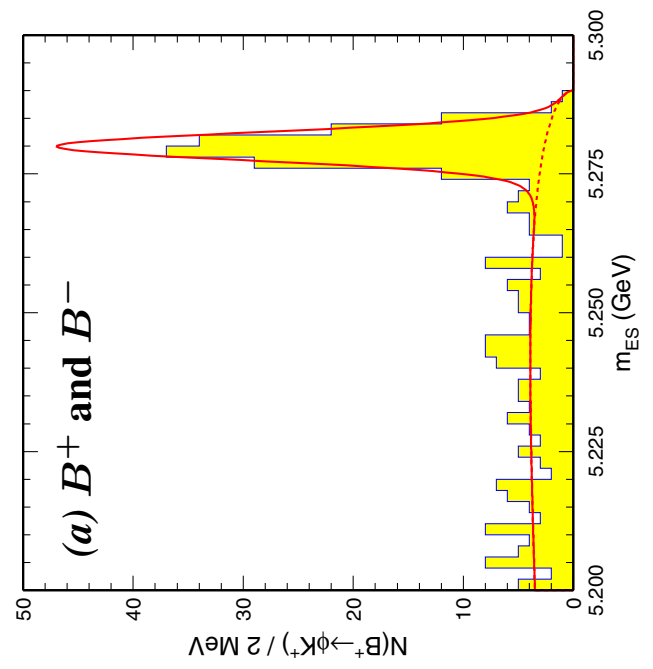

है

त)

$\stackrel{7}{7}$

$\cong$

ᄒํำ

:

흘

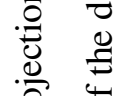

응

$\because \quad \frac{0}{\Xi}$

ప

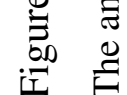




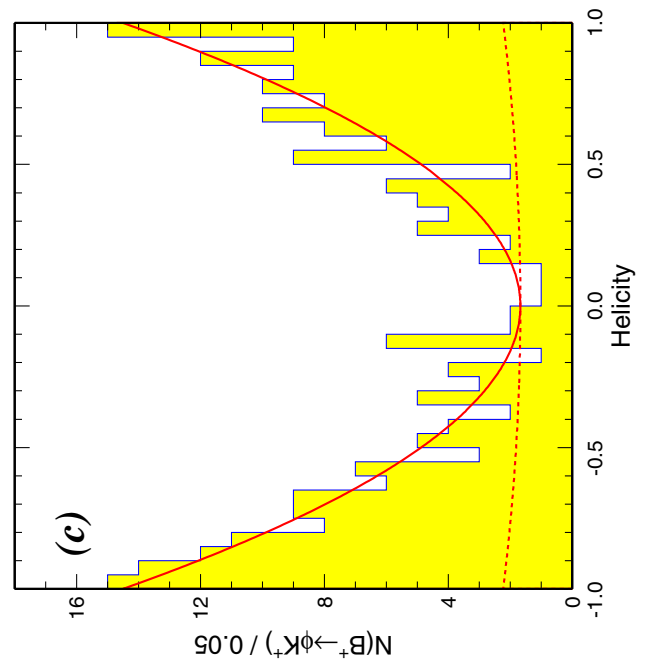

$\frac{1}{0}$

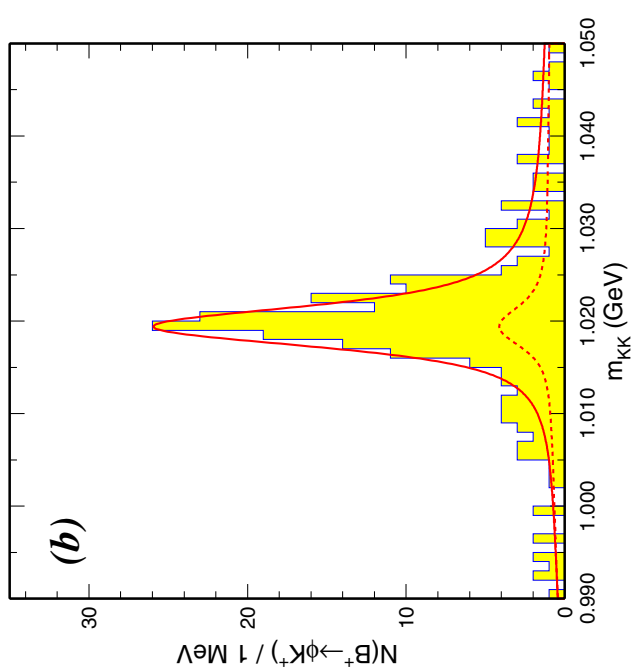

$+$

$\stackrel{\cap}{\Xi}$

s)

ర0

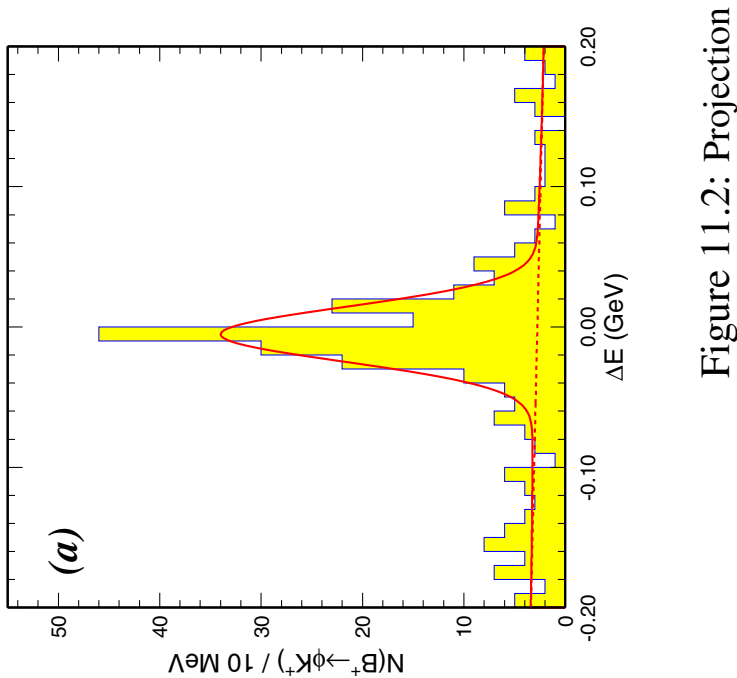



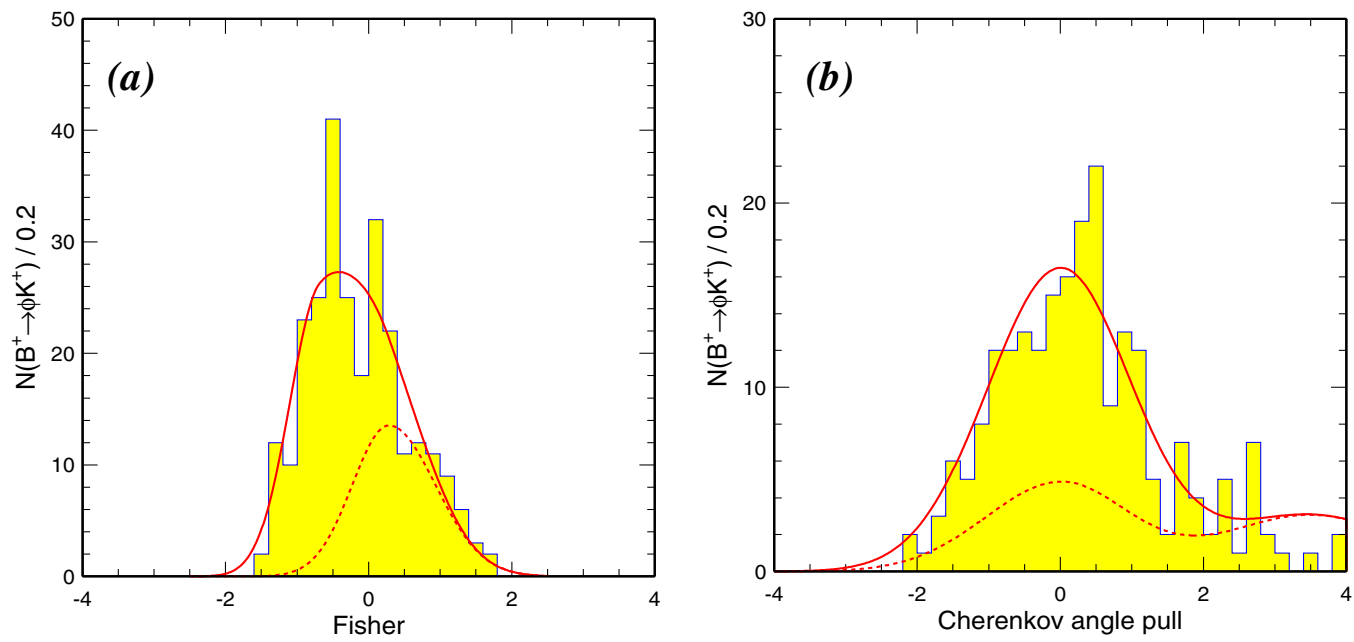

Figure 11.3: Projection plots for $(a)$ the Fisher discriminant $\mathcal{F}$ and $(b)$ the Cherenkov angle pull in $B^{ \pm} \rightarrow \phi K^{ \pm}$. The amount of expected background is estimated from sideband data.

\section{2 $\pi^{ \pm} / K^{ \pm}$separation in data}

The ability of our and other two-body and quasi-two-body analyses in BABAR to distinguish between decay modes with a "bachelor" (a. k. a. "prompt") charged track $B \rightarrow X \pi^{ \pm}$and $B \rightarrow X K^{ \pm}$rests on the difference between the $\Delta E$ and the DIRC Cherenkov angle pull values computed for the two track PID hypotheses. $d E / d x$ separation, which is good for tracks with lower momenta, provides little separation in the kinematic range of the bachelor tracks and is not used in this analysis; the DIRC separation is by far the most powerful of the three.

\subsection{1 $\Delta E \pi^{ \pm} / K^{ \pm}$separation}

It is straightforward to derive from Eq. 4.2 that for a reconstructed $B$ candidate, the separation $\Delta(\Delta E)=\Delta E_{K}-\Delta E_{\pi}$ between the $K^{ \pm}$and the $\pi^{ \pm}$hypotheses is 

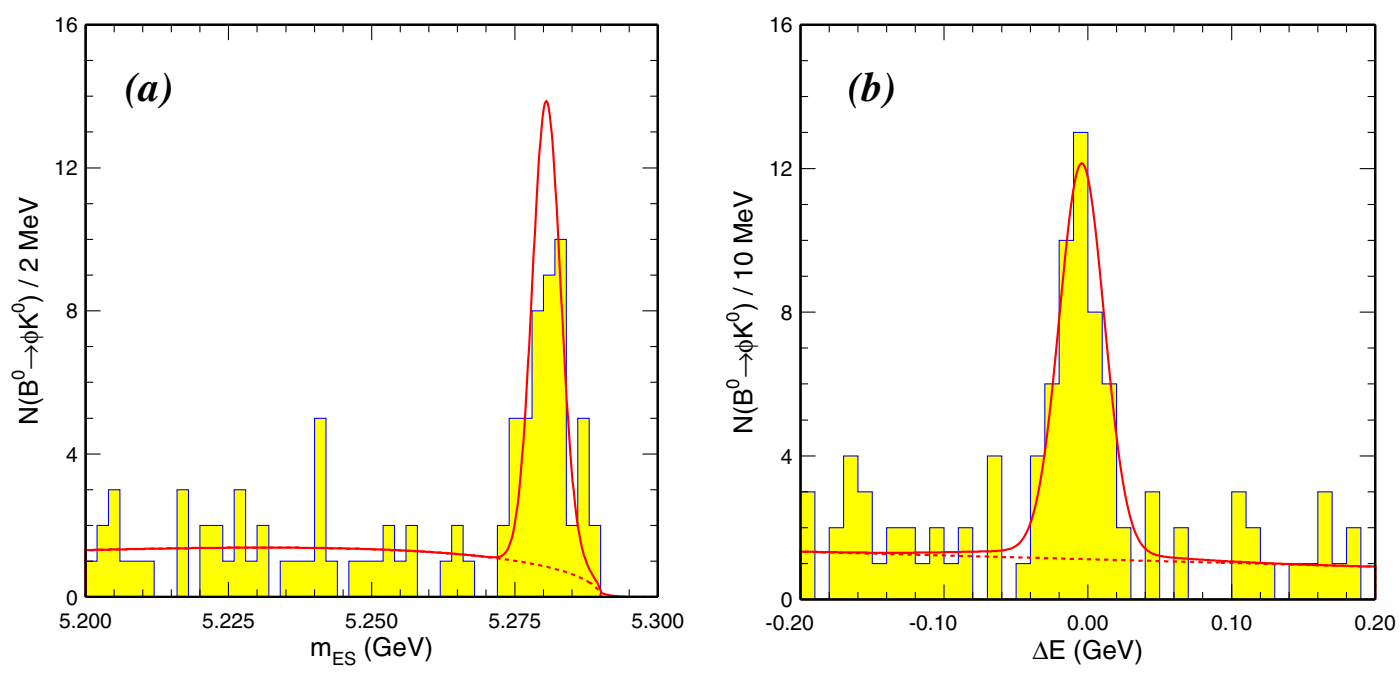

Figure 11.4: Projection plots for the variables (a) $m_{\mathrm{ES}}$ and $(b) \Delta E$ in $B^{0} \rightarrow \phi K_{S}^{0}$.
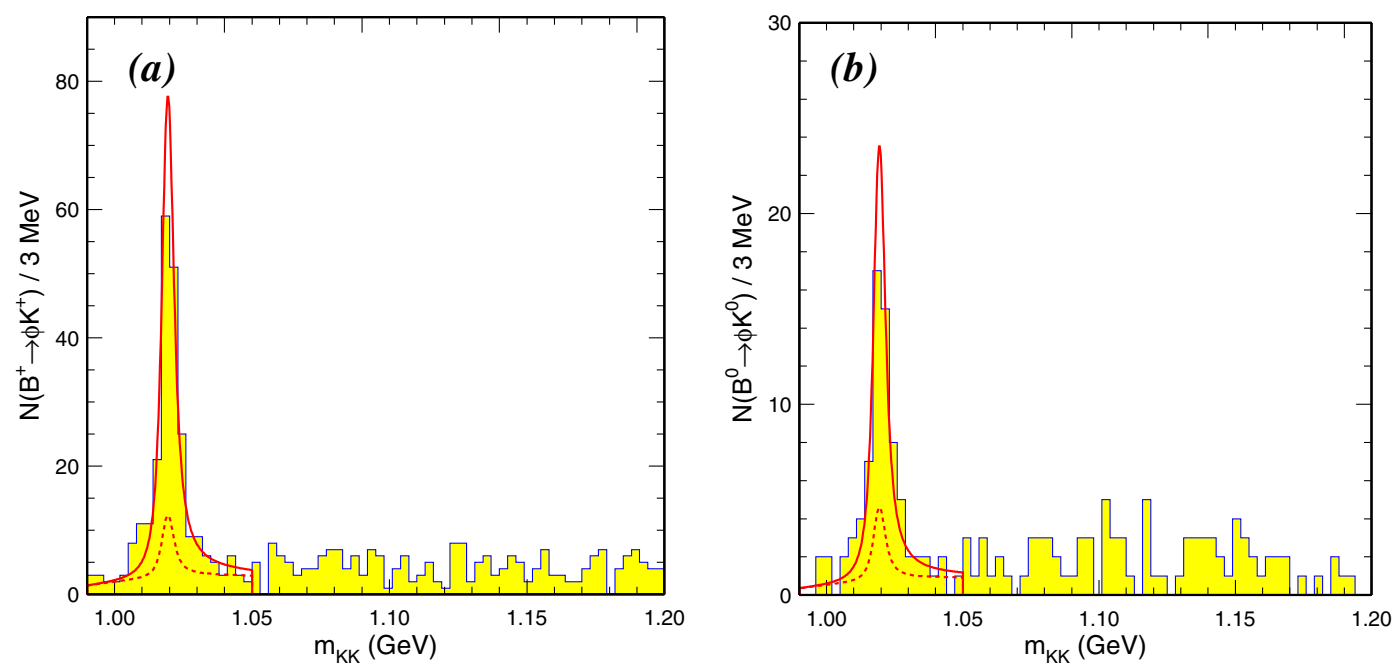

Figure 11.5: Projection plots for $m_{K^{+} K^{-}}$extended to $1.2 \mathrm{GeV} / c^{2}$, for $(a) B^{ \pm} \rightarrow \phi K^{ \pm}$and (b) $B^{0} \rightarrow \phi K_{S}^{0}$. No evidence is seen for a higher-resonance contribution under the $\phi$ peak. 

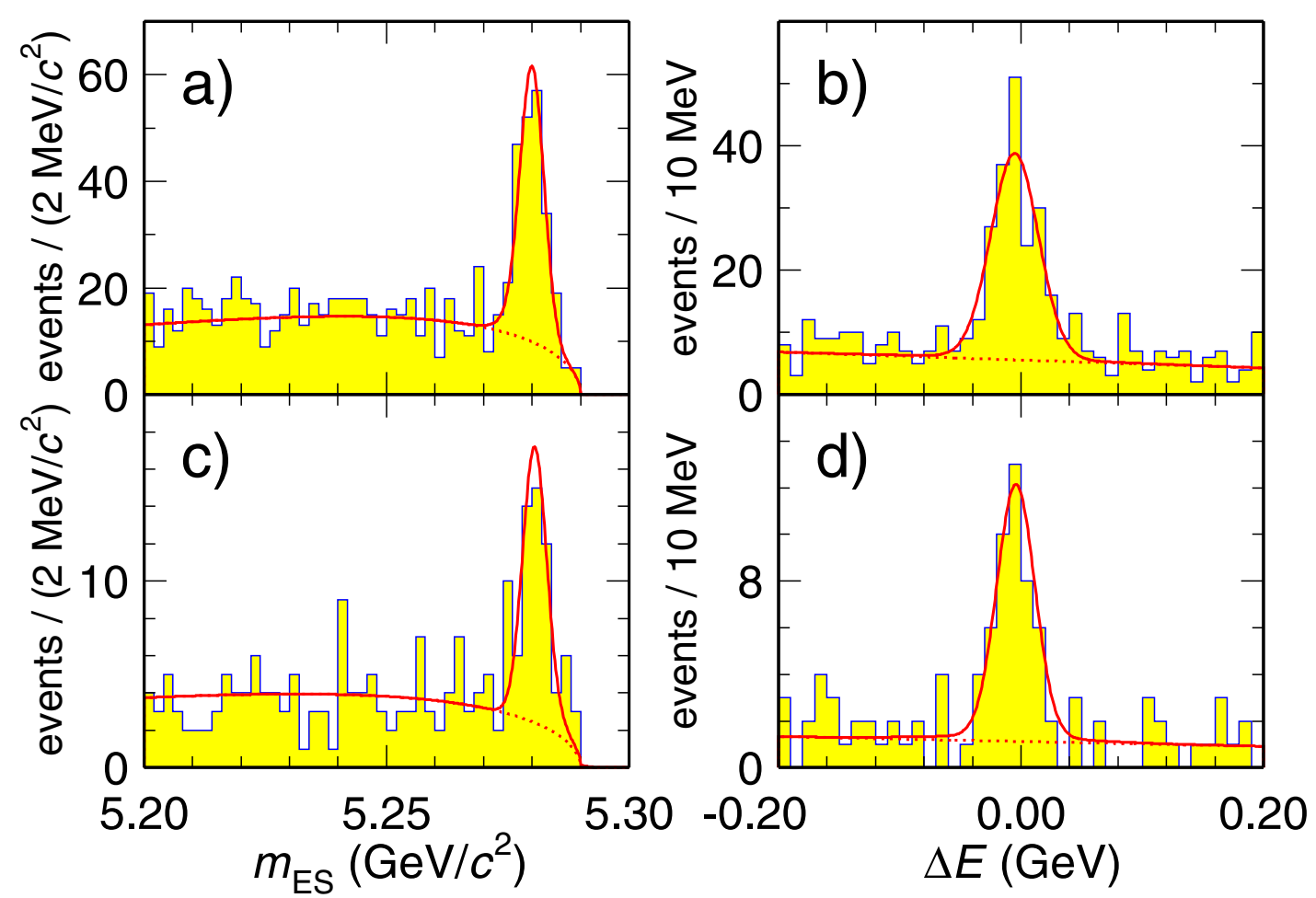

Figure 11.6: Projection plots of the variables $m_{\mathrm{ES}}(\mathrm{a}, \mathrm{c})$ and $\Delta E(\mathrm{~b}, \mathrm{~d})$ in the fit for the $\phi K^{+}$(top) and $\phi K_{S}^{0}\left(\pi^{+} \pi^{-}\right)$(bottom) branching fractions. The data are shown by the histogram, while the curve is the result of the fit. The signal-to-background ratio in the figure is enhanced with a requirement on the event probability that is less stringent than that in Eq. 11.1. 


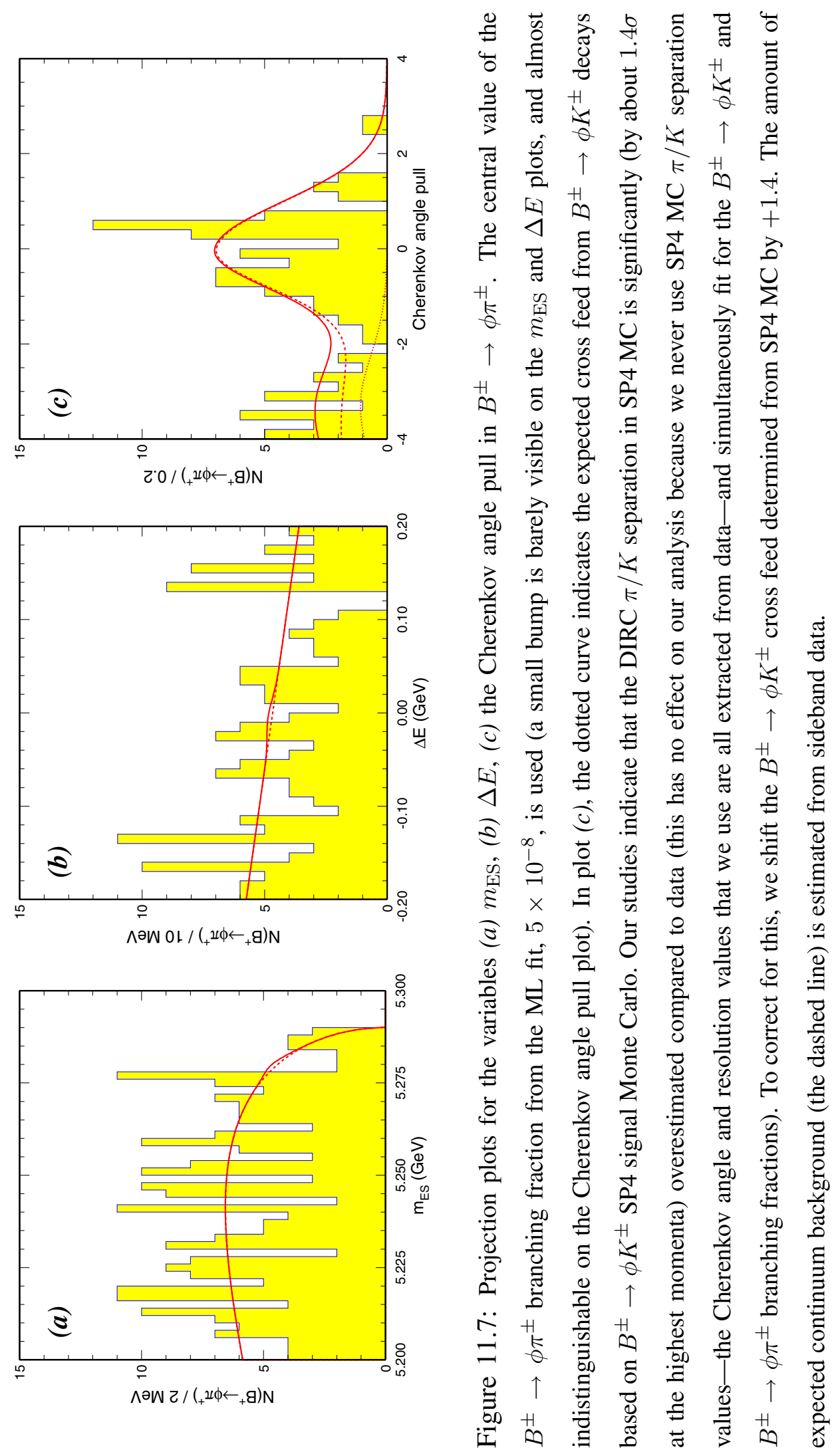




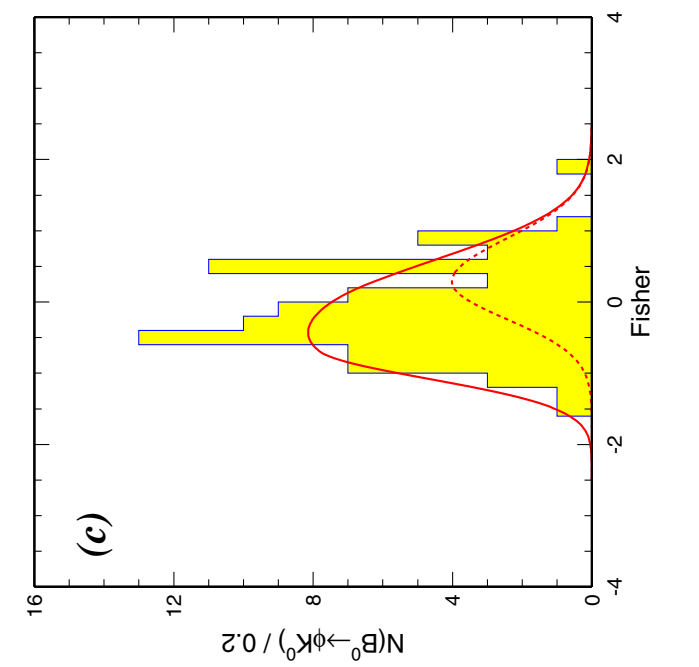

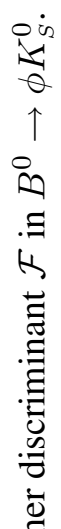

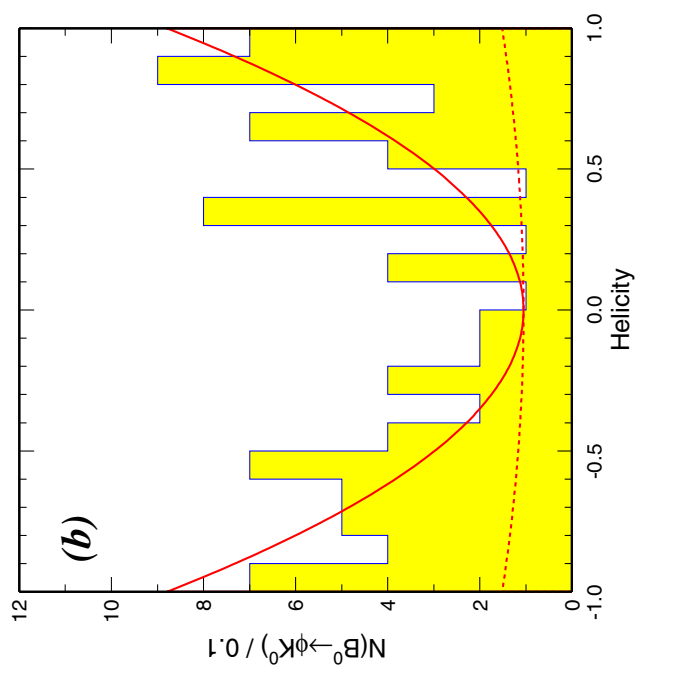

语 


$$
\Delta(\Delta E)=\gamma_{B_{A} B_{A R}} \times\left(\sqrt{m_{K}^{2}+p_{h^{ \pm}}^{2}}-\sqrt{m_{\pi}^{2}+p_{h^{ \pm}}^{2}}\right)
$$

where $\gamma_{B A B A R}=1.144$ is the value of the $\Upsilon(4 S)$ Lorentz boost factor in BABAR and $p_{h^{ \pm}}$ is the magnitude of the track's 3-momentum in the laboratory frame. For the decay $B^{ \pm} \rightarrow \phi h^{ \pm}$in $B A B A R$, the momenta of tracks that fall within the DIRC acceptance $\left(\vartheta_{\text {lab }} \in[0.45,2.45]\right)$ range approximately from $1.5 \mathrm{GeV}$ to $4.1 \mathrm{GeV}$, resulting in $\Delta E$ separation ranging from $31 \mathrm{MeV}$ for the fastest of the bachelor tracks to $88 \mathrm{MeV}$ for the slowest (the dependence of these numbers on the mass of $X$ in $B \rightarrow X h^{ \pm}$is rather weak). For a more detailed discussion on the $\Delta E \pi^{ \pm} / K^{ \pm}$separation in BABAR, see [69].

As an illustration, we present in Fig. 11.9(a) a plot that is identical in all respect to the $B^{ \pm} \rightarrow \phi K^{ \pm} \Delta E$ projection plot in Fig. 11.2(a), except that we plot the wrong-PID $\Delta E_{\pi}$ instead of $\Delta E_{K}$

\subsubsection{DIRC Cherenkov angle pull $\pi^{ \pm} / K^{ \pm}$separation}

We compute the DIRC Cherenkov angle pulls for the $\pi^{ \pm}$and $K^{ \pm}$hypotheses using the calibration technique developed in analysis [96]. Similarly to the $\Delta E$ separation, the DIRC $\pi^{ \pm} / K^{ \pm}$separation works best for lower-momentum tracks, as shown in Fig. 11.9(b) [70]. In Fig. 11.9(c), we show a scatter plot of DIRC Cherenkov angle pulls for the $K^{ \pm}$PID hypothesis versus the tracks' laboratory-frame momenta for the $B^{ \pm} \rightarrow \phi K^{ \pm}$candidates in data after application of the likelihood ratio cuts described in the previous section and for $\pi^{ \pm}$bachelor tracks in the $B^{+} \rightarrow \pi^{+} \bar{D}^{0}\left(\bar{D}^{0} \rightarrow K^{+} \pi^{-}\right)$ 


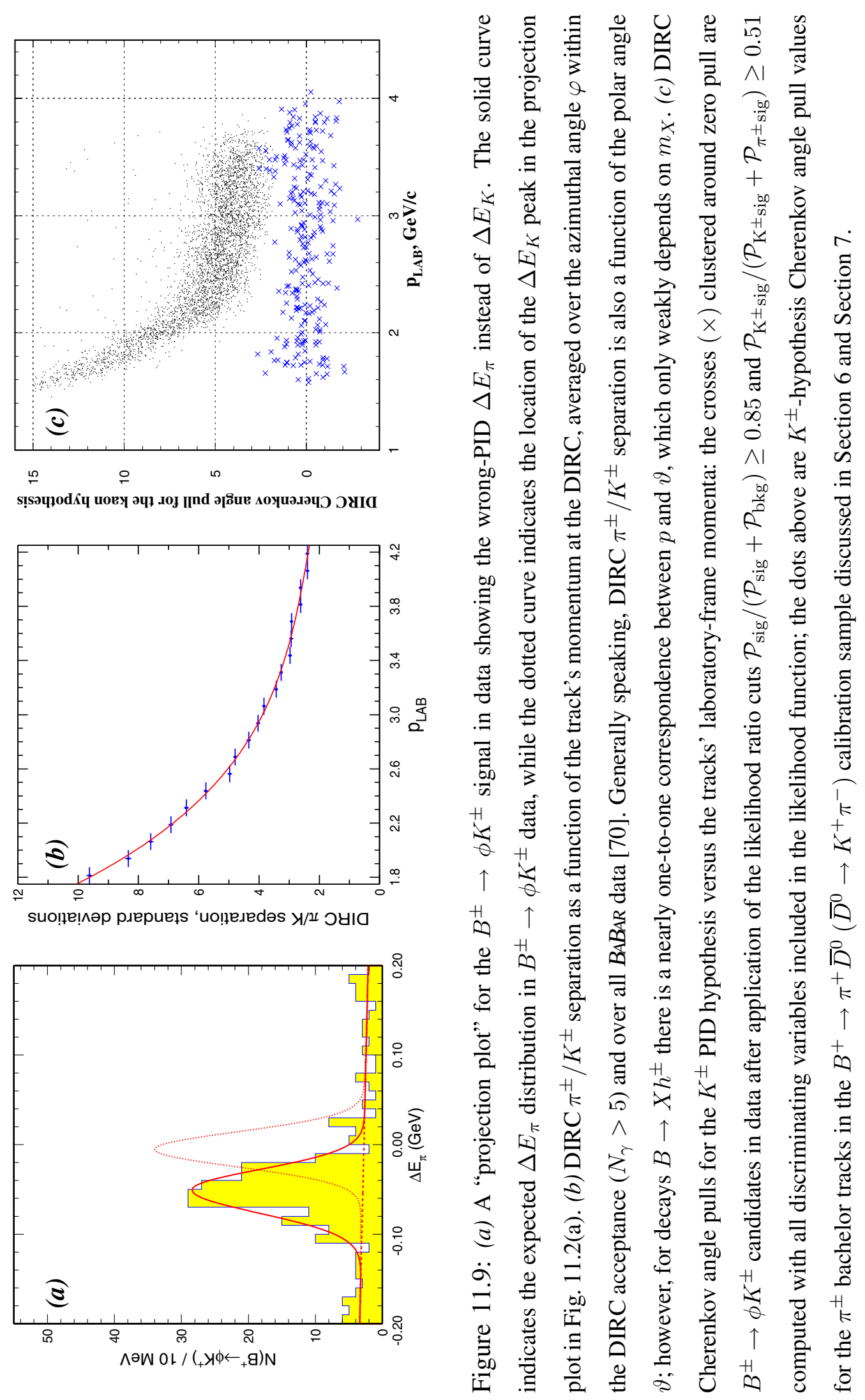


calibration sample discussed in Section 6 and Section 7. This plot demonstrates that we retain good $\pi^{ \pm} / K^{ \pm}$separation even at the highest bachelor track momenta available in $B^{ \pm} \rightarrow \phi h^{ \pm}$

\subsection{Off-resonance data sample and generic continuum Monte Carlo}

If everything is done correctly, the maximum-likelihood fit run on generic continuum $(u d s c \tau)$ Monte Carlo or off-resonance data should return a number of signal events that is consistent with zero. We found this to be the case in a test with $5.6 \mathrm{fb}^{-1}$ of offresonance data and an amount of generic continuum Monte Carlo roughly equivalent to a half of the Winter 2001 on-resonance data set.

\subsection{Generic $B \bar{B}$ Monte Carlo and the Charmless $B \bar{B}$ cocktail}

The $B \bar{B}$ background to most charmless channels currently under study in $B A B A R$ has been shown to be negligible. We analyzed $161.6 \times 10^{6}$ generic $B \bar{B}$ SP4 MC events, which is close to twice the number of $B \bar{B}$ pairs in the present $B A B A R$ data set, and $11.9 \times$ $10^{6}$ generic charmless $B \bar{B}$ events, which is roughly equivalent to $240 \mathrm{fb}^{-1}$ at $\Upsilon(4 S)$, or almost three times the size of the present $B A B A R$ data set. For each event passing all 
ML fit preselection cuts and a sufficiently high signal-to-background probability ratio $\left(\mathcal{P}_{\text {sig }} /\left(\mathcal{P}_{\text {sig }}+\mathcal{P}_{\text {bkg }}\right)>0.85\right.$ and, additionally, $\mathcal{P}_{\mathrm{K}^{ \pm} \text {sig }} /\left(\mathcal{P}_{\mathrm{K}^{ \pm}}{ }_{\text {sig }}+\mathcal{P}_{\pi^{ \pm} \text {sig }}\right)>0.5$ for $B^{ \pm} \rightarrow$ $\phi K^{ \pm}$and $\mathcal{P}_{\pi^{ \pm} \text {sig }} /\left(\mathcal{P}_{\mathrm{K}^{ \pm} \text {sig }}+\mathcal{P}_{\pi^{ \pm} \text {sig }}\right)>0.5$ for $\left.B^{ \pm} \rightarrow \phi \pi^{ \pm}\right)$, we retrieved and inspected the event's Monte Carlo Truth decay tree. A summary of our findings follows.

\subsection{1 $B \bar{B}$ background to $B^{ \pm} \rightarrow \phi K^{ \pm}$}

We found no events emulating the decay $B^{ \pm} \rightarrow \phi K^{ \pm}$in our sample of $80.9 \mathrm{M}$ generic $B^{0} \bar{B}^{0} \mathrm{SP} 4 \mathrm{MC}$ events. The sample of $80.7 \mathrm{M}$ generic $B^{+} B^{-}$events was found to contain

- 278 true $B^{ \pm} \rightarrow \phi K^{ \pm}$events,

- 45 non-resonant $B^{ \pm} \rightarrow K^{+} K^{-} K^{ \pm}$events,

- $3 B^{ \pm} \rightarrow \phi \pi^{ \pm}$events.

Analysis of $11.9 \mathrm{M} B \bar{B}$ events in which one of the $B$ 's was forced to decay hadronically into a state with no charm quark revealed

- 421 true $B^{ \pm} \rightarrow \phi K^{ \pm}$events,

- 41 non-resonant $B^{ \pm} \rightarrow K^{+} K^{-} K^{ \pm}$events,

- $5 B^{ \pm} \rightarrow \phi \pi^{ \pm}$events, and

- $1 B^{0} \rightarrow \phi K^{* 0}, K^{* 0} \rightarrow \pi^{-} K^{+}$event.

The number of observed true $B^{ \pm} \rightarrow \phi K^{ \pm}$events, which were included in SP4 Monte Carlo production with a branching fraction of $10 \times 10^{-6}$, is entirely consistent with our expectations. It is also not a problem, as was demonstrated in Table 9.1, that 
a few $B^{ \pm} \rightarrow \phi \pi^{ \pm}$events (which were included in SP4 Monte Carlo production with a branching fraction of $1 \times 10^{-6}$ ) pass through our rather arbitrary pair of probability ratio cuts.

It is well known that in SP4 Monte Carlo, the rate of non-resonant 3-body $B$-meson decays, which are generated via the JETSET generator, was far too excessive and is not to be trusted. Later in this Section, we will present results of a study with large exclusive samples of $B^{ \pm} \rightarrow K^{+} K^{-} K^{ \pm}, B^{ \pm} \rightarrow K^{+} K^{-} \pi^{ \pm}$and $B^{0} \rightarrow K^{+} K^{-} K_{S}^{0}$ events that establishes an upper limit on the contribution of non-resonant 3-body decays to the $B \rightarrow \phi h$ branching fractions that are under study in this paper. The $B \rightarrow \phi K^{*}$ decays are also subjected to an exclusive analysis later in this Section.

\subsection{2 $B \bar{B}$ background to $B^{ \pm} \rightarrow \phi \pi^{ \pm}$}

A straight ML fit for the $B^{ \pm} \rightarrow \phi h^{ \pm}$decay modes to the full generic $B^{0} \bar{B}^{0} \mathrm{MC}$ set finds $0 B^{ \pm} \rightarrow \phi K^{ \pm}$and $2 B^{ \pm} \rightarrow \phi \pi^{ \pm}$events. The probability ratio cuts described above are passed by $4 B^{ \pm} \rightarrow \phi \pi^{ \pm}$-like events, of which 3 are random track combinations and 1 is a $B^{0} \rightarrow \phi K_{S}^{0}$ event.

The full set of generic $B^{+} B^{-} \mathrm{MC}$ was found to contain

- 35 true $B^{ \pm} \rightarrow \phi \pi^{ \pm}$events,

- 37 non-resonant $B^{ \pm} \rightarrow K^{+} K^{-} \pi^{ \pm}$events,

- $3 B^{ \pm} \rightarrow \phi K^{ \pm}$events.

The charmless $B \bar{B}$ cocktail has 
- 39 true $B^{ \pm} \rightarrow \phi \pi^{ \pm}$events,

- 43 non-resonant $B^{ \pm} \rightarrow K^{+} K^{-} \pi^{ \pm}$events,

- $3 B^{ \pm} \rightarrow \phi K^{ \pm}$events,

- $3 B^{0} \rightarrow \phi K_{S}^{0}$ events.

- $1 B^{+} \rightarrow \pi^{+} K^{* 0}, K^{* 0} \rightarrow \pi^{-} K^{+}$event.

The possible cross feed of $B^{0} \rightarrow \phi K_{S}^{0}$ and $B^{+} \rightarrow h^{+} K^{* 0}, K^{* 0} \rightarrow \pi^{-} K^{+}$into $B^{ \pm} \rightarrow \phi h^{ \pm}$will be discussed separately. The other types of backgrounds have already been addressed in the previous section.

\subsection{3 $B \bar{B}$ background to $B^{0} \rightarrow \phi K_{s}^{0}$}

Generic $B^{+} B^{-}$decays have not been able to produce anything resembling $B^{0} \rightarrow \phi K_{S}^{0}$. Generic $B^{0} \bar{B}^{0}$ decays were found to contain

- 110 true $B^{0} \rightarrow \phi K_{S}^{0}$ events (present with a branching fraction of $5 \times 10^{-6}$ in SP4 $\mathrm{MC})$,

- 15 non-resonant $B^{0} \rightarrow K^{+} K^{-} K_{S}^{0}$ events,

- 6 'bonus' JETSET-generated $B^{0} \rightarrow \phi K_{S}^{0}$ events (this is a known bug in SP4 MC),

- $1 B^{0} \rightarrow \phi K^{* 0}, K^{* 0} \rightarrow \pi^{0} K_{S}^{0}$ event.

Finally, the charmless $B \bar{B}$ cocktail has

- 168 true $B^{0} \rightarrow \phi K_{S}^{0}$ events,

- 22 non-resonant $B^{0} \rightarrow K^{+} K^{-} K_{S}^{0}$ events, and 
- 13 JETSET-generated $B^{0} \rightarrow \phi K_{S}^{0}$ events.

\subsubsection{Conclusion of the generic $B \bar{B}$ MC study}

The most important conclusion of this study is that the amount of random-track background to $B \rightarrow \phi h$ that is due to $B \bar{B}$ events is essentially zero, and that all observed events that are not true signal come from a handful of $B$ decay modes with a similar topology. Let's now examine each of these modes in a greater detail.

\subsection{Exclusive $B$ decay modes: $B \rightarrow \phi K^{*}, 3$-body, etc.}

In Table 11.1, we present the fractions (in units of one-thousandth) of events from about a dozen $B$ decay modes that cross feed into the modes $B^{ \pm} \rightarrow \phi K^{ \pm}, B^{ \pm} \rightarrow \phi \pi^{ \pm}$or $B^{0} \rightarrow \phi K_{S}^{0}$. We start with exclusive MC samples of several tens of thousands of events (over a million for $K^{+} K^{-} K^{ \pm}$and $K^{+} K^{-} \pi^{ \pm}$), so the statistical errors are small.

\subsubsection{Cross feed from $B \rightarrow \phi K^{*}$ modes}

The numbers of $B \rightarrow \phi K^{*}$ events that are expected to pass the preselection cuts are 5.6, or $3.2 \%$ of the event yield, for $B^{ \pm} \rightarrow \phi h^{ \pm}$and 9.1 , or $18 \%$ of the event yield for $B^{0} \rightarrow \phi K_{S}^{0}$. However, the distributions of ML fit variables in these decay modes differ significantly from the signal PDFs, which is evidenced by the fact that the ML fitter run on pure $B \rightarrow \phi K^{*}$ signal MC classifies only a small fraction of these events as $B \rightarrow$ $\phi h$ signal. The background dilution effect described on page 101 further reduced the 


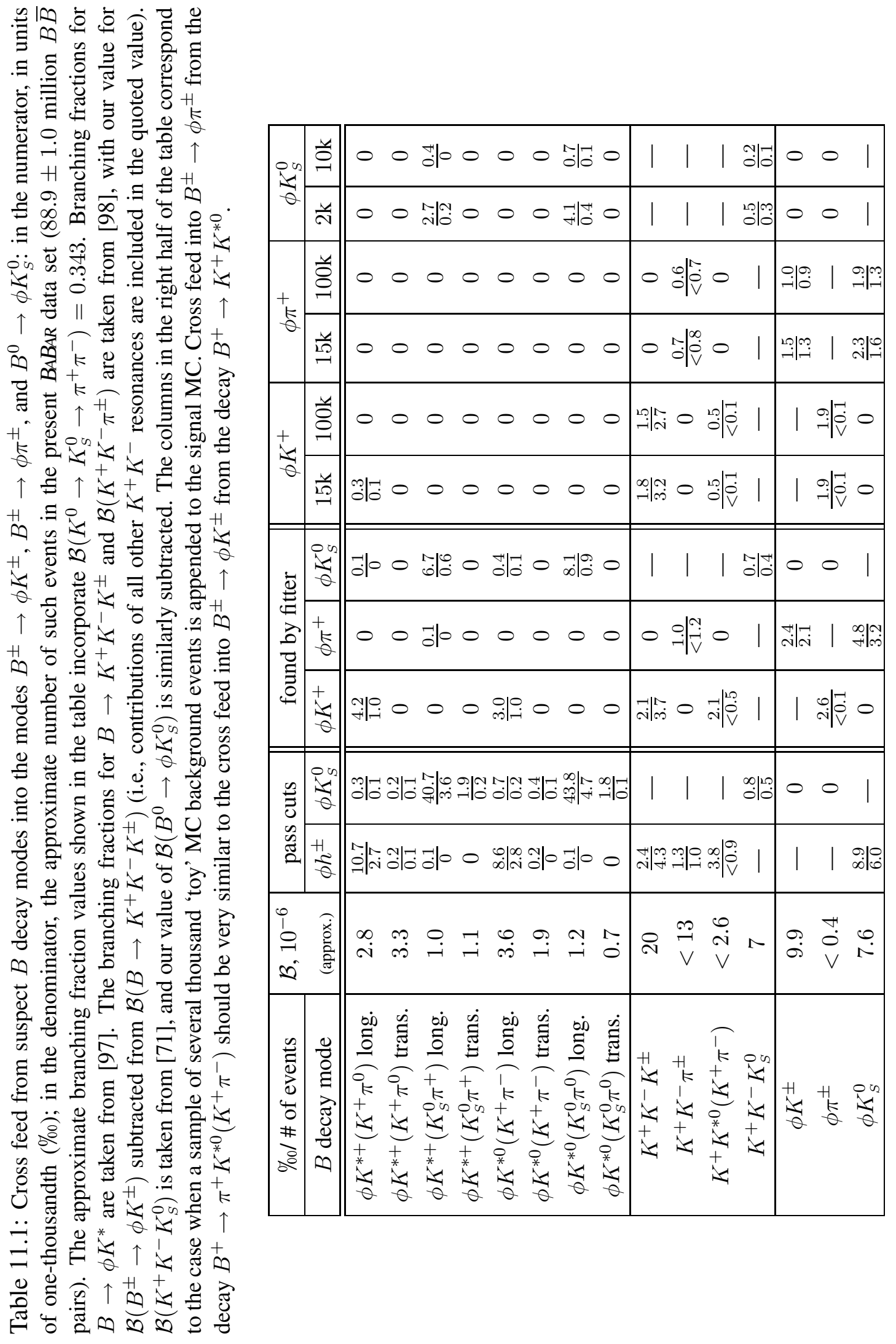


expected cross feed from $B \rightarrow \phi K^{*}$ to the negligible $<0.1$ events for both $B^{ \pm} \rightarrow \phi h^{ \pm}$ and $B^{0} \rightarrow \phi K_{S}^{0}$.

\subsubsection{Cross feed from non-resonant three-body decays}

In the amplitudes of these decays interfere with the $B^{ \pm} \rightarrow \phi h^{ \pm}, B^{0} \rightarrow \phi K_{S}^{0}$ amplitudes in the vicinity of the $\phi$ resonance. Since $\phi$ is a very narrow resonance, the contribution of the interference cross-term to the decay rate vanishes.

Since these decays are completely reconstructed (unlike $B \rightarrow \phi K^{*}$, where one of the final-state particles is lost), the distributions of ML fit variables for these decays are more signal-like. Indeed, the numbers of events found by the ML fitter either in pure three-body signal MC or in signal MC mixed with 'toy' continuum background are only slightly smaller than the corresponding numbers of events passing the preselection cuts.

An important issue to mention here is that the three-body branching fractions quoted in the table include contributions from the $K^{+} K^{-}$resonant structure above the $\phi$. In particular, the shape of the $K \bar{K}$ mass distribution around $1.5 \mathrm{GeV}$ that is reported in [71] is consistent with a broad scalar resonance; the number of events in that region indicates that the true non-resonant three-body $K \bar{K} K$ branching fractions are at least twice, or even thrice, smaller than the values quoted in the table, making the $K^{+} K^{-} K_{S}^{0}$ cross feed into $\phi K_{S}^{0}$ negligible. We estimate the irreducible non-resonant $K^{+} K^{-} K^{ \pm}$background to the decay $B^{ \pm} \rightarrow \phi K^{ \pm}$to be $<1.3$ events, or $<0.8 \%$. Our determination of the $K^{+} K^{-} \pi^{ \pm}$background to $B^{ \pm} \rightarrow \phi \pi^{ \pm}$is $<0.3$ events, or $<5 \%$ of 6.5 events, the the 90\% CL upper limit on the $B^{ \pm} \rightarrow \phi \pi^{ \pm}$event yield. 


\subsubsection{Cross feed of $B \rightarrow \phi h$ modes into each other}

There is no cross feed of $B^{ \pm} \rightarrow \phi \pi^{ \pm}$into $B^{ \pm} \rightarrow \phi K^{ \pm}$because $\mathcal{B}\left(B^{ \pm} \rightarrow \phi \pi^{ \pm}\right)$is very small, and there is no measurable cross feed from any of the $B^{ \pm} \rightarrow \phi h^{ \pm}$modes into $B^{0} \rightarrow \phi K_{S}^{0}$. There is, however, a non-negligible cross feed into the $B^{ \pm} \rightarrow \phi \pi^{ \pm}$event yield from both $B^{ \pm} \rightarrow \phi K^{ \pm}$and $B^{0} \rightarrow \phi K_{S}^{0}$, estimated at $<2.2$ events, or $<34 \%$ of the $90 \% \mathrm{CL}$ upper limit on the $B^{ \pm} \rightarrow \phi \pi^{ \pm}$event yield.

\section{6 $B \bar{B}$ background: conclusion}

Having conducted exhaustive studies of the possible $B \bar{B}$ background to the three branching fractions that are the subject of this paper, we find that the only non-negligible source of systematic error is the $K^{+} K^{-} K^{ \pm}$background to the decay $B^{ \pm} \rightarrow \phi K^{ \pm}$, estimated to be $<1.3$ events, or $<0.8 \%$. Still, to remain conservative, we will quote a systematic error of $1 \%$ for all three $B \rightarrow \phi h$ modes.

In principle, $B \bar{B}$ background is a source of an additive systematic error. If we were using the cut-and-count approach, we would be able to perform background subtraction and thus reduce the upper limit on $\mathcal{B}\left(B^{ \pm} \rightarrow \phi \pi^{ \pm}\right)$by about a third. However, with the maximum-likelihood approach, we can only place an upper bound on the amount of $B \bar{B}$ background. Since the presence of $B \bar{B}$ background can bias the event yield only upward, the $90 \%$ CL upper limit on the $B^{ \pm} \rightarrow \phi \pi^{ \pm}$event yield requires no adjustment due to this source of systematic error. 


\section{7 "Goodness-of-fit" tests}

Thorough "goodness-of-fit" tests have been performed to confirm that the values of likelihood $\mathcal{L}$ obtained in the fits to on-resonance data are consistent with MC-based expectations.

Using simulated events only (randomly chosen SP4 Monte Carlo events for signal and "toy Monte Carlo" (PDF-based) events for background), we construct a large number (200 for $B^{ \pm} \rightarrow \phi K^{ \pm}$and 500 for $B^{0} \rightarrow \phi K_{S}^{0}$ ) of mock data samples that contain the same numbers of signal and background events as those reported by the maximumlikelihood fit (see Tables 10.1 and 10.2). We run the fitter on each of these mock data samples and plot the distribution of $-2 \ln \mathcal{L}$.

If the PDFs are representative of data, one expects the value of $-2 \ln \mathcal{L}$ obtained in the fit to on-resonance data to lie within a few standard deviations of the mean of this distribution. The results were satisfactory: in both modes, the difference was under one standard deviation. 


\section{Chapter 12}

\section{Systematic Studies}

Our measurement of the $B$ decay branching fractions and charge asymmetries has uncertainties other than pure statistical errors on $N_{\text {sig. }}$. Most of the sources of systematic errors were discussed in previous sections of this document. Those that were not are discussed below. A summary of all non-negligible sources of systematic uncertainty is given in table 12.1.

\subsection{Uncertainty of PDF parameters}

First, we discuss uncertainties in the ML fit. When we perform the ML fit to obtain the number of signal events, we make certain assumptions about the signal and background distributions. We know the uncertainties on the parameters used in the fit, but we fix them to the most probable value in the fit. In order to account for this uncertainty, we have to vary the parameters within their errors. 
We perform a large number (typically, 1000) of fit experiments when all PDF parameters are varied within their errors. The errors are assumed to be Gaussian and independent. However, in some cases they are correlated: for example, in the double Gaussian parametrization, the mean and the widths are correlated, and we use the covariance matrix. In each experiment we obtain a likelihood function, which provides the number of signal events, significance, and upper limit. We histogram their values and find the systematic error due to the PDF parameters from the event yield variation.

We also use PDF variation experiments for Monte Carlo convolution of the likelihood function where we assume Gaussian resolution functions for PDF parameters. We sum the likelihood distributions from each PDF variation experiment and obtain the likelihood function which includes systematics in the PDF. This procedure is the Monte Carlo realization of the convolution

$$
\mathcal{L}_{\text {syst }}\left(N_{\text {sig }}\right)=\int d \vec{f} d \vec{g} \mathcal{L}\left(N_{\text {sig }} \mid \vec{f}, \vec{g}\right) \mathcal{P}\left(\vec{f}, \vec{g} \mid \overrightarrow{f_{0}}, \vec{g}_{0}\right)
$$

where $\vec{f}_{0}$ and $\vec{g}_{0}$ are the nominal values (best estimates) of the PDF parameters $\vec{f}$ and $\vec{g}$, and $\mathcal{P}\left(\vec{f}, \vec{g} \mid \vec{f}_{0}, \vec{g}_{0}\right)$ is their Gaussian distribution.

We derive significance and $90 \%$ CL upper limit values with systematics from the summed likelihood distribution $\left(\mathcal{L}_{\text {syst }}\left(N_{\text {sig }}\right)\right.$ in Eq. 12.1), which is broader than the original without systematics included. This results in a larger value of the upper limit, smaller significance, and larger total errors. In some cases the central value (likelihood maximum value) may shift slightly relative to the nominal one, resulting in either 
smaller upper limit or higher significance (but not both). However, this effect is small and we always take the more conservative value. When we quote a central value for our measurement we always obtain it from the nominal fit (from $\mathcal{L}\left(N_{\text {sig }} \mid \vec{f}_{0}, \vec{g}_{0}\right)$ in Eq. 12.1), but include the systematic errors from the PDF uncertainties.

We determine that the systematic errors on the branching fractions that arise from the uncertainty of PDF parameters are $\pm 2.0 \%$ for $B^{ \pm} \rightarrow \phi K^{ \pm}$and $\pm 2.8 \%$ for $B^{0} \rightarrow \phi K_{S}^{0}$. Additionally, the rms of the $B^{ \pm} \rightarrow \phi \pi^{ \pm}$yield is 0.73 events, or $10.9 \%$ of the $90 \% \mathrm{CL}$ upper limit of 6.7 events.

\subsection{The systematic error on $\mathcal{A}_{C P}\left(B^{ \pm} \rightarrow \phi K^{ \pm}\right)$}

The systematic error on $\mathcal{A}_{C P}\left(B^{ \pm} \rightarrow \phi K^{ \pm}\right)$is small because most sources of systematics contribute equally to both $B^{+}$and $B^{-}$event yields and thus cancel out from $\mathcal{A}_{C P}$.

The systematic errors on the charge asymmetries $\mathcal{A}_{C P}$ in $B^{ \pm} \rightarrow \phi K^{ \pm}$that are due to PDF parameter uncertainties were obtained following the procedure described in the previous section: $\pm 0.47 \%$ for the signal, $\pm 0.25 \%$ for the $K^{ \pm}$component of the continuum background, and $\pm 0.16 \%$ for the $\pi^{ \pm}$component of the continuum background.

Reference [88] reports that the charge asymmetry in tracking in the present $B A B A R$ data set (averaged over the DCH acceptance, with an unspecified momentum distribution) is $(0.24 \pm 0.25) \%$, where the sign is chosen such that a positive value means a higher efficiency for positive tracks (the reverse of Eq. 5.2). We cannot use this result directly because the tracking charge asymmetry for the bachelor track momentum distri- 
Table 12.1: Summary of sources of systematic uncertainties to $B \rightarrow \phi h$ branching fractions (in percent).

\begin{tabular}{|c|c|c|c|}
\hline \multirow[b]{2}{*}{ Source of uncertainty } & \multicolumn{2}{|c|}{$\mathcal{B}\left(B^{ \pm} \rightarrow \phi h^{ \pm}\right)$} & \multirow[b]{2}{*}{$\mathcal{B}\left(B^{0} \rightarrow \phi K^{0}\right)$} \\
\hline & $\mathcal{B}\left(B^{ \pm} \rightarrow \phi K^{ \pm}\right)$ & $\operatorname{UL} \mathcal{B}\left(B^{ \pm} \rightarrow \phi \pi^{ \pm}\right)$ & \\
\hline PDF parameters & 2.0 & 10.9 & 2.8 \\
\hline$B \bar{B}$ counting & \multicolumn{2}{|c|}{1.1} & 1.1 \\
\hline Daughter $\mathcal{B}$ & \multicolumn{2}{|c|}{1.4} & 1.5 \\
\hline Monte Carlo statistics & \multicolumn{2}{|c|}{0.7} & 0.7 \\
\hline ML fit efficiency & \multicolumn{2}{|c|}{1.0} & 1.0 \\
\hline Tracking efficiency & \multicolumn{2}{|c|}{2.4} & 4.2 \\
\hline PID in $\phi \rightarrow K^{+} K^{-}$ & \multicolumn{2}{|c|}{2.0} & 2.0 \\
\hline$K_{S}^{0}$ efficiency & \multicolumn{2}{|c|}{ - } & 2.0 \\
\hline Track multiplicity cut & \multicolumn{2}{|c|}{1.0} & 1.0 \\
\hline$\left|\cos \theta_{T}\right|$ cut & \multicolumn{2}{|c|}{1.0} & 1.0 \\
\hline DIRC $N_{\text {phot }}$ cut & \multicolumn{2}{|c|}{1.0} & 1.0 \\
\hline$B \bar{B}$ background & 1.0 & - & 1.0 \\
\hline Total & 4.7 & 11.7 & 6.5 \\
\hline
\end{tabular}


bution found in $B^{ \pm} \rightarrow \phi K^{ \pm}$may be noticeably different. However, we do have within our analysis two independent cross-checks of the tracking charge asymmery:

- The average charge asymmetry in the continuum background to $B^{ \pm} \rightarrow \phi h^{ \pm}$(see Table 10.1 on page 109) is $(-0.27 \pm 0.86) \%$.

- The charge asymmetry in the high-statistics calibration mode $B^{+} \rightarrow \pi^{+} \bar{D}^{0}\left(\bar{D}^{0} \rightarrow\right.$ $\left.K^{+} \pi^{-}\right)$(Section 7) is $(-2.2 \pm 1.4) \%$.

A substantially more extentensive study of the tracking charge asymmetry in BABAR has been undertaken and is described in detail in [97]. In addition, members of the TwoBody Analysis Working Group have conducted a study of the DIRC charge asymmetry in decays with charged tracks in the same kinematic range as in $B^{ \pm} \rightarrow \phi h^{ \pm}$(see, for example, [89], [96]). We find that the combined systematic error on $\mathcal{A}_{C P}$ due to charge asymmetries in tracking and the DIRC is less than $1.0 \%$.

Finally, the charge-dependent tracking bias in runs 18000-22929, which was discussed in Section 7.2 (page 74), contributes a systematic error of $\pm 0.1 \%$ to $\mathcal{A}_{C P}$ (and a negligible systematic error to the $B \rightarrow \phi h$ branching fractions). To obtain the value of this systematic error, we varied by \pm one, and then by \pm several standard deviations the value of $C$ used in correcting the momenta of bachelor tracks in the affected run range and compared the values of signal $\mathcal{A}_{C P}$ returned by the ML fit.

We combine this number with the systematic error due to PDF uncertainties to obtain the total systematic error on $\mathcal{A}_{C P}\left(B^{ \pm} \rightarrow \phi K^{ \pm}\right): 1.1 \%$. 


\subsection{Other sources of systematic errors}

The other sources of systematic error not discussed elsewhere in this document are the small data/MC differences in the efficiencies of the track multiplicity, $\left|\cos \theta_{T}\right|$ and DIRC

$N_{\text {phot }}$ cuts. It is standard practice to estimate all three to be smaller than $1 \%$ (e.g., see $[91,97,99])$. 


\section{Chapter 13}

\section{Other Direct- $C P$ Results from $B_{A} B_{A} A$}

The high $B$-meson production rate of the BABAR and Belle experiments allows them to measure direct- $C P$ asymmetries in a substantial number of charmless $B$-meson decay modes. As in $B^{ \pm} \rightarrow \phi K^{ \pm}$, the values of $\mathcal{A}_{C P}$ predicted within the Standard Model for each of these decay modes are small, typically below $1 \%$.

We present in Tables 13.1 and 13.2 a summary of latest results (including preliminary results) on direct $C P$ violation from $B A B A R$, Belle and CLEO that was prepared in August 2003 by the Heavy Flavor Averaging Group [72]. The only mode with a worldaveraged $C P$ asymmetry that shows evidence $(3.4 \sigma)$ for not being consistent with zero is $B^{0} \rightarrow K^{+} \pi^{-}$. 


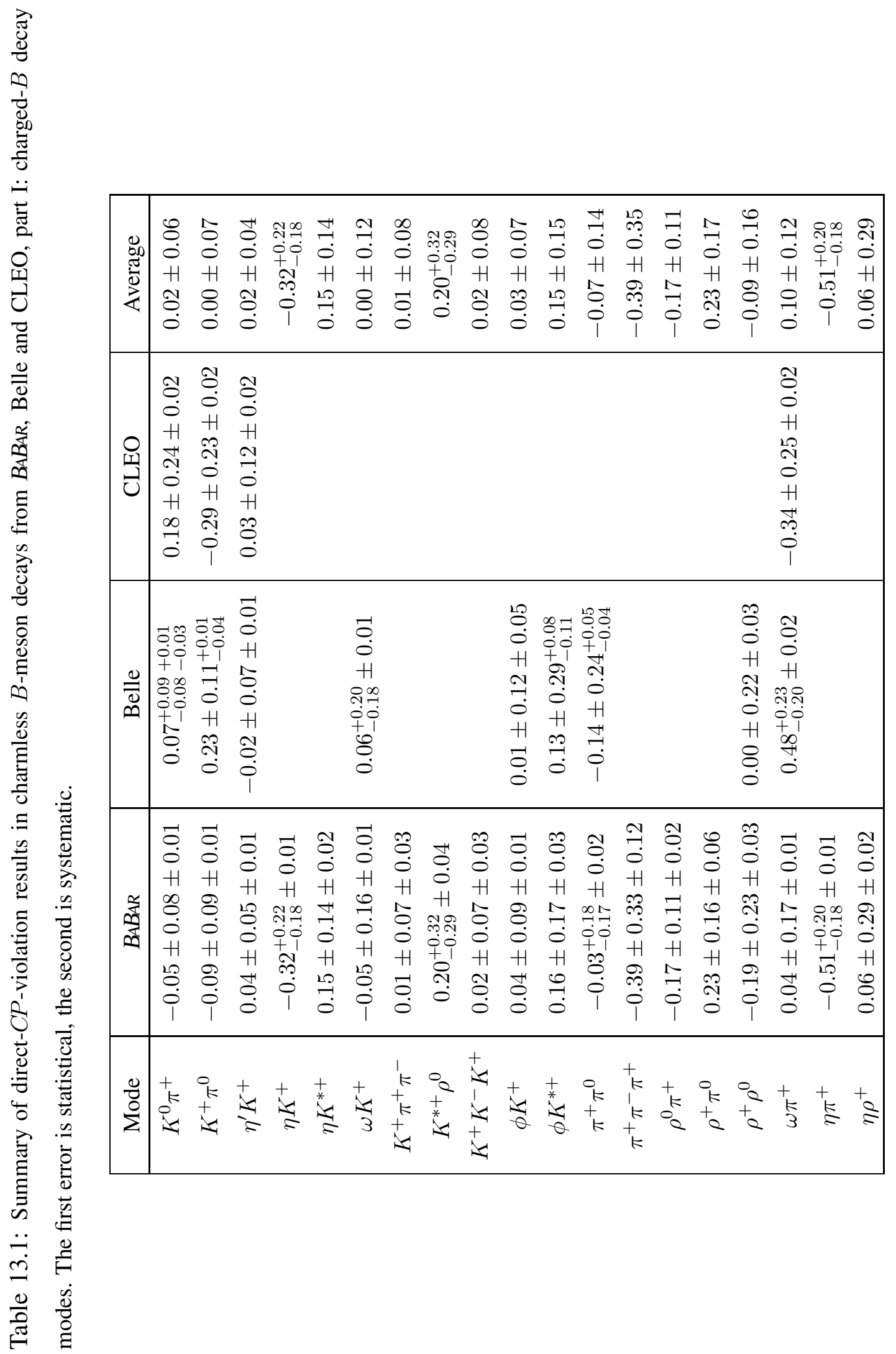




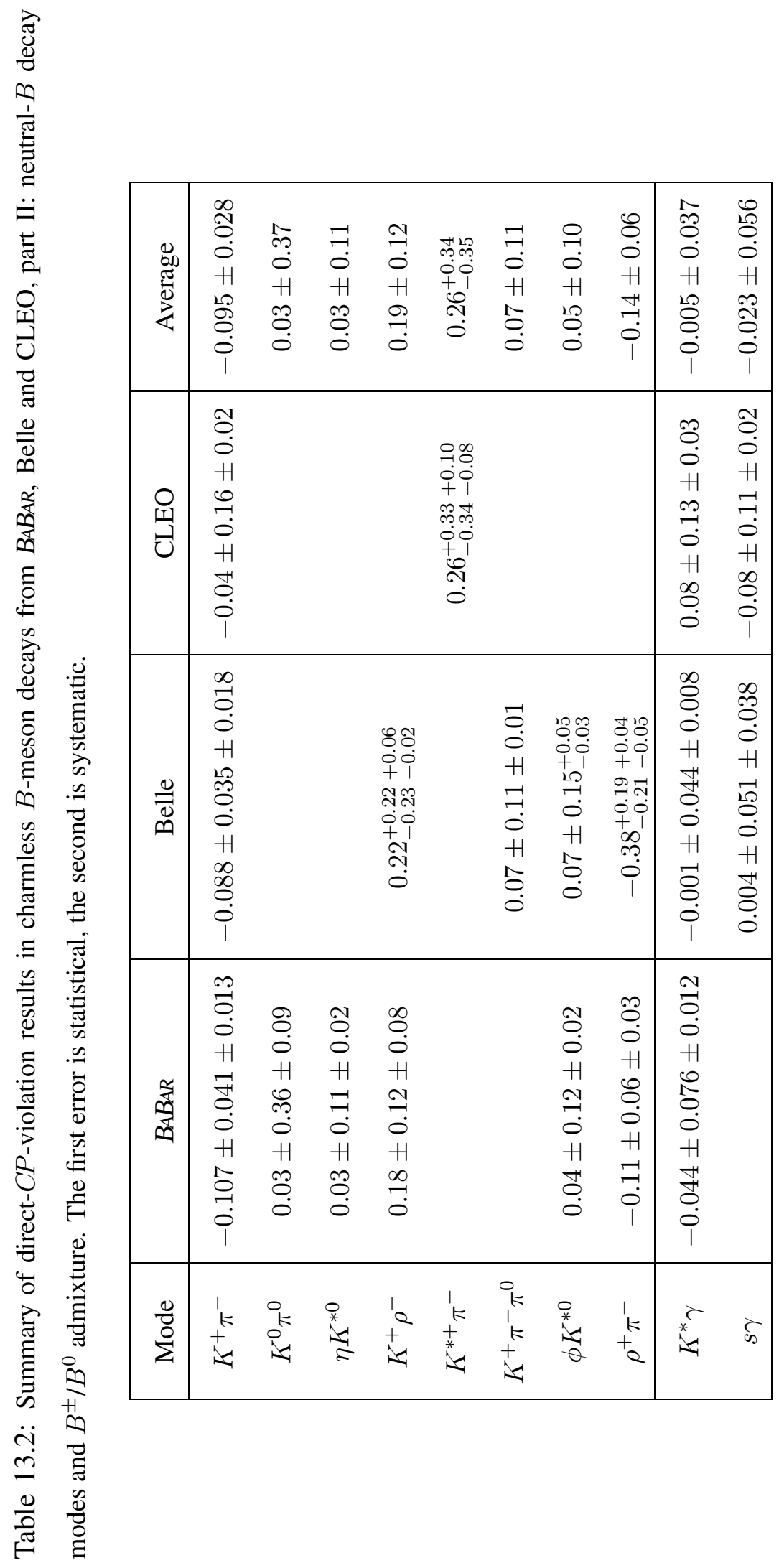




\section{Chapter 14}

\section{Summary}

We performed measurements of branching fractions and charge asymmetries in the $b \rightarrow$ $s \bar{s} s$ penguin-dominated decays decays $B^{+} \rightarrow \phi K^{+}$and $B^{0} \rightarrow \phi K^{0}$ in a sample of approximately 89 million $B \bar{B}$ pairs (“BABAR Summer 2002 dataset”). We have determined $\mathcal{B}\left(B^{+} \rightarrow \phi K^{+}\right)=\left(10.0_{-0.8}^{+0.9} \pm 0.5\right) \times 10^{-6}$ and $\mathcal{B}\left(B^{0} \rightarrow \phi K^{0}\right)=\left(8.4_{-1.3}^{+1.5} \pm 0.5\right) \times 10^{-6}$. Additionally, we measure the $C P$-violating charge asymmetry $\mathcal{A}_{C P}\left(B^{ \pm} \rightarrow \phi K^{ \pm}\right)=$ $0.04 \pm 0.09 \pm 0.01$, with a $90 \%$ confidence-level interval of $[-0.10,0.18]$, and set an upper limit on the CKM- and color-suppressed decay $B^{+} \rightarrow \phi \pi^{+}, \mathcal{B}\left(B^{+} \rightarrow \phi \pi^{+}\right)<$ $0.41 \times 10^{-6}$ (at the $90 \%$ confidence level).

Our results are consistent with the predictions of the Standard Model, which predicts $\mathcal{A}_{C P}\left(B^{ \pm} \rightarrow \phi K^{ \pm}\right) \lesssim 1 \%$ and $\mathcal{B}(B \rightarrow \phi \pi) \ll 10^{-7}$. A substantial fraction of the parameter space of existing new-physics models has thus been excluded. 


\section{Appendix A}

\section{Miscellanea}

This Appendix serves as a repository for terminology and definitions the majority of our readers should be familiar and comfortable with, yet a reminder or precise formulation of which may be necessary.

\section{A.1 Terminology of Experimental Particle Physics}

- Thrust: A measure of "jettiness" (or, if you wish, "two-jettiness") of a set of $N$ particles, or of the extent to which these particles are aligned along a common axis. The vector of unit length $\vec{A}$ that maximizes the value of thrust $T$ is the thrust axis of the set:

$$
T=\max \frac{\sum_{i=1}^{N}\left|\vec{A} \cdot \overrightarrow{p_{i}}\right|}{\sum_{i=1}^{N} \sqrt{\left|\overrightarrow{p_{i}}\right|^{2}}},
$$

where vectors $\overrightarrow{p_{i}}$ are usually taken in the collider's center-of-mass frame. Thrust $T$ 
assumes values from $1 / 2$, which would correspond to a perfectly spherical event with an infinite number of tracks, to 1 , which corresponds to two back-to-back tracks, such as in $e^{+} e^{-} \rightarrow \mu^{+} \mu^{-}$. There is a two-fold ambiguity in the direction of $\vec{A}$, which we resolve by requiring that the projection of $\vec{A}$ on the $Z$ axis be positive.

- Continuum background: background that is due to processes whose cross sections do not peak in the vicinity of the $\Upsilon(4 S)$ resonance, namely due to $e^{+} e^{-}$production of $u \bar{u}, d \bar{d}, s \bar{s}$ and $c \bar{c}$ pairs and products of their fragmentation and decay. $e^{+} e^{-} \rightarrow \tau^{+} \tau^{-}$is usually also included in the notion of continuum background, but $e^{+} e^{-} \rightarrow \mu^{+} \mu^{-}$and $e^{+} e^{-} \rightarrow e^{+} e^{-}$are not.

\section{- PID: Particle IDentification.}

- Ntuple (also spelled " $n$-tuple"): an ordered set of $n$-dimensional elements, where each of the dimensions is a variable of a fixed type (real, integer, boolean, etc.). Your checkbook register is an example of an ntuple with variables that correspond to the check number, date, amount, the name of the payee, the remaining balance, etc. In a typical analysis, creation of data files that contain one or several ntuples with entries for events that pass a predefined set of cuts is one of the data-reduction steps. 


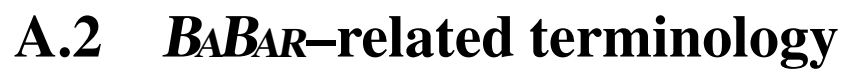

- SVT: The 5-layer Silicon Vertex Tracker, the innermost part of the BABAR detector.

- DCH: The Drift Chamber, BABAR's primary tracking system.

- DIRC: "Detection of Internally Reflected Cherenkov Radiation", a novel fused silica (synthetic quartz) detector, which is BABAR's primary source of charged particle PID information.

- EMC: The CsI(Tl) Electro-Magnetic Calorimeter. Measures energy of electromagnetic showers produced by photons and charged particles. The primary source of electrons and positrons veto information for our analysis.

- IFR: The Instrumented Flux Return, BABAR's primary muon and $K_{L}^{0}$ ID system. Our analysis does not use IFR information.

- On- and off-resonance: the nominal center-of-mass energy of the PEP-II collider is at the peak of the $\Upsilon(4 S)$ resonance, $\sqrt{s}=10.5800 \mathrm{GeV}$. Roughly $12 \%$ of all BABAR data is collected $40 \mathrm{MeV}$ below the $\Upsilon(4 S)$, i. e., approximately $20 \mathrm{MeV}$ below the $B \bar{B}$ production threshold. Off-resonance data is a clean sample of $u d s c \tau$ continuum background.

- SP4: Simulation Production 4, production of Monte Carlo-simulated events that began in mid-2001.

- BAD : "BABAR Analysis Document". Access to most BADs is usually restricted 
to BABAR members and associates. BADs intended for distribution outside the Collaboration will normally have no references to other BADs. However, such references are unavoidable in a dissertation.

- BABAR coordinate system : The $Z$ axis points roughly in the direction of the electron beam at the IP, $Y$ points up, and $X$ points toward the center of the PEP-II rings. Because of the design requirements of the final focus system, the beams at the IP are tilted by approximately $-19 \mathrm{mrad}$ in the $X Z$ plane.

\section{A.3 Functions}

- ARGUS function: A phenomenological function introduced by the ARGUS experiment [64] that happens to describe very well the shape of $m_{\mathrm{ES}}$ distribution observed in continuum background to $B$ decays:

$$
\operatorname{ARGUS}\left(x ; \xi, E_{\text {beam }}\right)=\operatorname{Norm} \times x \sqrt{1-\left(x / E_{\text {beam }}\right)^{2}} \exp \left(\xi\left[1-\left(x / E_{\text {beam }}\right)^{2}\right]\right)
$$

Throughout our analysis, the value of $E_{\text {beam }}=\sqrt{s} / 2$ is fixed at $5.2900 \mathrm{GeV}$.

- Double Gaussian: a sum of two Gaussian distributions, each with its own mean, sigma, and norm. The six parameters that we use are: (1) AREA, the total area under the double Gaussian curve; (2) MEAN, the mean of the first Gaussian; (3) SIGMA1, the sigma of the first Gaussian; (4) AR2/AREA, the fraction of AREA that 
is due to the second Gaussian; (5) DELM, the mean of the second Gaussian being MEAN+DELM, and (6) SIG2/SIG1, the ratio of sigmas of the second and the first Gaussians.

- Bifurcated Gaussian: A Gaussian with different sigmas on the left and on the right of the mean.

- PDF: Probability Density (or Distribution) Function.

\section{A.4 Theoretical description of resonance shapes}

Neglecting interference with nearby resonances, ${ }^{1}$ the shape of a resonance of nominal mass $M_{0}$ and nominal width $\Gamma_{0}$ is described by the relativistic Breit-Wigner function [62]

$$
B W(M)=\frac{M \cdot M_{0} \cdot \Gamma(M)}{\left(M_{0}^{2}-M^{2}\right)^{2}+M_{0}^{2} \Gamma^{2}(M)},
$$

where the resonance's width is itself a function of mass,

$$
\Gamma(M)=\Gamma_{0} \frac{M_{0}}{M}\left(\frac{q}{q_{0}}\right)^{2 \ell+1} \cdot \frac{W_{\ell}^{\prime 2}(q)}{W_{\ell}^{\prime 2}\left(q_{0}\right)} .
$$

Here $\ell$ is the resonance's spin, $q$ is the momentum of its decay products in the resonance's rest frame, $q_{0}$ is the momentum at $M=M_{0}$, and $W_{\ell}^{\prime}(q)$ are the often neglected Blatt-Weisskopf damping factors [63, 85, 73]

\footnotetext{
${ }^{1}$ and Bose-Einstein correlations, which are only important in consideration of the $\rho$ resonance
} 


$$
\begin{array}{r}
W_{0}^{\prime}(q)=1, \\
W_{1}^{\prime}(q)=\sqrt{\frac{2}{1+(q R)^{2}}}, \\
W_{2}^{\prime}(q)=\sqrt{\frac{13}{\left[(q R)^{2}-3\right]^{2}+9(q R)^{2}}}, \\
W_{4}^{\prime}(q)=\sqrt{\frac{W_{3}^{\prime}(q)=\sqrt{\frac{277}{(q R)^{2}\left[(q R)^{2}-15\right]^{2}+9\left[2(q R)^{2}-5\right]}}}{\left[(q R)^{4}-45(q R)^{2}+105\right]^{2}+25(q R)^{2}\left[2(q R)^{2}-21\right]^{2}}},
\end{array}
$$

where $R$ is the interaction radius of the strong force. Von Hippel and Quigg [63] quote as plausible the interaction radii of 0.25 to $0.75 \mathrm{fm}\left(10^{-13} \mathrm{~cm}\right)$ for meson resonances and 0.5 to $1.0 \mathrm{fm}$ for baryon resonances. 


\section{Appendix B}

\section{Input-variable correlation matrices}

In this appendix, we present the correlation matrices for the maximum-likelihood fit variables used in the analyses describes in this note.

It is well known that $\left(m_{\mathrm{ES}}, \Delta E\right)$ is a pair of weakly, but non-vanishingly correlated kinematic variables. An important feature of this pair of variables is that the correlation between them in the continuum background is essentially zero. It has been previously demonstrated in other BABAR analyses that the effect of the small $\sim-10 \%$ correlation between $m_{\mathrm{ES}}$ and $\Delta E$ on the determination of branching fractions is negligible.

\section{B.1 Correlation matrices in $B^{ \pm} \rightarrow \phi h^{ \pm}$}

Is natural that the bachelor charged track Cherenkov angle pulls for the pion and the kaon hypotheses are strongly correlated, as are $\Delta E$ and the Cherenkov angle pulls. Both $\Delta E$ and the Cherenkov angle pulls are used to distinguish between $B^{ \pm} \rightarrow \phi K^{ \pm}$ 
Table B.1: Correlation matrix for $B^{ \pm} \rightarrow \phi K^{ \pm}$SP4 signal Monte Carlo (18303 events).

\begin{tabular}{|c|ccccccc|}
\hline & $\mathcal{F}$ & $\Delta E$ & $m_{\mathrm{ES}}$ & $m_{K K}$ & $\operatorname{pull}_{K}$ & $\operatorname{pull}_{\pi}$ & $\mathcal{H}$ \\
\hline $\mathcal{F}$ & 1 & & & & & & \\
$\Delta E$ & -0.02 & 1 & & & & & \\
$m_{\mathrm{ES}}$ & 0.00 & -0.11 & 1 & & & & \\
$m_{K K}$ & -0.01 & 0.02 & -0.01 & 1 & & & \\
$\operatorname{pull}_{K}$ & 0.01 & -0.05 & 0.00 & -0.01 & 1 & & \\
$\operatorname{pull}_{\pi}$ & -0.07 & 0.43 & -0.01 & -0.01 & 0.33 & 1 & \\
$\mathcal{H}$ & -0.03 & 0.01 & 0.01 & -0.01 & -0.02 & 0.00 & 1 \\
\hline
\end{tabular}

and $B^{ \pm} \rightarrow \phi \pi^{ \pm}$; their correlations are appropriately accounted for in the likelihood function used in our analysis.

\section{B.2 Correlation matrices in $B^{0} \rightarrow \phi K_{S}^{0}$}


Table B.2: Correlation matrix for $B^{ \pm} \rightarrow \phi K^{ \pm}$on-resonance data (14371 events).

\begin{tabular}{|c|ccccccc|}
\hline & $\mathcal{F}$ & $\Delta E$ & $m_{\mathrm{ES}}$ & $m_{K K}$ & $\operatorname{pull}_{K}$ & $\operatorname{pull}_{\pi}$ & $\mathcal{H}$ \\
\hline $\mathcal{F}$ & 1 & & & & & & \\
$\Delta E$ & -0.04 & 1 & & & & & \\
$m_{\mathrm{ES}}$ & -0.02 & 0.00 & 1 & & & & \\
$m_{K K}$ & 0.02 & 0.01 & 0.00 & 1 & & & \\
$\operatorname{pull}_{K}$ & 0.09 & -0.06 & -0.03 & 0.01 & 1 & & \\
$\operatorname{pull}_{\pi}$ & 0.01 & 0.03 & -0.01 & 0.00 & 0.62 & 1 & \\
$\mathcal{H}$ & 0.01 & 0.01 & 0.01 & 0.01 & -0.00 & 0.00 & 1 \\
\hline
\end{tabular}

Table B.3: Correlation matrix for $B^{0} \rightarrow \phi K_{S}^{0}$ SP4 signal Monte Carlo (18451 events).

\begin{tabular}{|c|ccccc|}
\hline & $\mathcal{F}$ & $\Delta E$ & $m_{\mathrm{ES}}$ & $m_{K K}$ & $\mathcal{H}$ \\
\hline $\mathcal{F}$ & 1 & & & & \\
$\Delta E$ & -0.01 & 1 & & & \\
$m_{\mathrm{ES}}$ & 0.00 & -0.10 & 1 & & \\
$m_{K K}$ & 0.00 & 0.03 & -0.01 & 1 & \\
$\mathcal{H}$ & 0.01 & -0.01 & -0.01 & 0.01 & 1 \\
\hline
\end{tabular}


Table B.4: Correlation matrix for $B^{0} \rightarrow \phi K_{S}^{0}$ on-resonance data (2043 events).

\begin{tabular}{|c|ccccc|}
\hline & $\mathcal{F}$ & $\Delta E$ & $m_{\mathrm{ES}}$ & $m_{K K}$ & $\mathcal{H}$ \\
\hline $\mathcal{F}$ & 1 & & & & \\
$\Delta E$ & -0.04 & 1 & & & \\
$m_{\mathrm{ES}}$ & -0.04 & -0.01 & 1 & & \\
$m_{K K}$ & 0.02 & 0.00 & -0.01 & 1 & \\
$\mathcal{H}$ & -0.02 & 0.03 & -0.01 & 0.00 & 1 \\
\hline
\end{tabular}




\section{Appendix C}

\section{$K_{S}^{0} \rightarrow \pi^{+} \pi^{-}$Reconstruction}

\section{Efficiency in $B_{A} B_{A} A$ : Release-10 Data}

\section{vs. SP4 Monte Carlo}

\section{C.1 "Executive summary"}

We present results of a study of relative $K_{S}^{0}$ reconstruction efficiencies in Release-10 data and SP4 Monte Carlo. A large semi-inclusive sample of $K_{S}^{0}$ candidates is used, 1.4 million in data and 2.0 million in MC. We subdivide the samples into a large number of bins in momentum, polar angle and transverse $(X Y) K_{S}^{0}$ flight length; bin-by-bin ratios normalized to the $[0.3 \mathrm{~cm}, 1.3 \mathrm{~cm}]$ bin in the $X Y K_{S}^{0}$ flight length and their respective statistical errors are then computed; the difference between the $K_{S}^{0}$ mass resolutions in data and $\mathrm{MC}$ is also taken into account. The resulting $K_{S}^{0}$ reconstruction efficiency 
correction tables are applied to signal Monte Carlo for the modes $B^{0} \rightarrow \phi K_{S}^{0}$ and $B^{0} \rightarrow$ $\pi^{+} D^{-}\left(D^{-} \rightarrow K_{S}^{0} \pi^{-}\right)$, which provide $K_{S}^{0}$ spectra representative of most cases of $K_{S}^{0}$ use in $B A B A R$ analyses, to determine the overall correction factor and its statistical error. The above exercise is repeated for several sets of $K_{S}^{0}$ quality cuts, from none to tight, and for three different binning approaches in order to determine the systematic error. For these modes we are able to determine the data/MC $K_{S}^{0}$ reconstruction efficiency correction with a statistical error of $\sim 1 \%$; the systematic error has been determined to be under $1.5 \%$. Since no BABAR analysis (with the notable exception of $B^{0} \rightarrow \psi K_{S}^{0}$ ) would benefit from knowing the value of the data/MC $K_{S}^{0}$ reconstruction efficiency correction with a better than $\sim 3 \%$ precision, our study has achieved its goal. We have made our $K_{S}^{0}$ efficiency correction tables publicly accessible; collaborators are welcome to follow the recipe provided in this note to compute the overall correction factor for their analyses.

\section{C.2 Introduction}

A large number of branching-fraction analyses in $B A B A R$ involve reconstruction of the decay $K_{S}^{0} \rightarrow \pi^{+} \pi^{-}$, where the two charged pions belong to the list ChargedTracks of all reconstructed tracks in the event. In order to determine the desired branching fraction properly, one has to know, among other things, the ratio of the $K_{S}^{0}$ reconstruction efficiencies in data and Monte Carlo. The MC correction tables [88] that existed for about two years have recently been determined to be incorrect, which caused some of BABAR's preliminary results to become public with a drastically conservative $10 \%$ systematic er- 
ror on the $K_{S}^{0}$ reconstruction efficiency. The purpose of this study is to fill the void and to allow several analyses to advance to publication as soon as possible.

The Tracking Efficiency Task Force has determined that for ChargedTracks originating within $15 \mathrm{~mm}$ in $X Y$ from the beam spot the ratio of the track reconstruction efficiencies in Release-10 data and SP4 MC is $1.000 \pm 0.005$ [88]. However, most $K_{S}^{0}$ 's decay outside this $15 \mathrm{~mm}$ radius, where various factors can make this ratio different from unity. In general, the value of this ratio may be a function of the location of the $K_{S}^{0}$ decay vertex within the detector, as well as the $K_{S}^{0}$ momentum.

Our approach is to choose an appropriate binning in $K_{S}^{0}$ momentum, direction of flight (polar angle $\theta_{\text {lab }}$ only) and transverse flight distance $d_{X Y}$, determine the numbers of $K_{S}^{0}$ 's in each of the bins in data and $\mathrm{MC}$, and, for each of the momentum and polar angle ranges, normalize the ratio of these numbers to its value in the first bin in $d_{X Y}$, where all tracking effects are believed to be understood and thus the value of the ratio is 1.000 by definition, with no associated error other than the $0.5 \%$ per track systematic error mentioned in the previous paragraph ${ }^{1}$.

In order to reduce this statistical error, we eliminate most of the combinatorial (random-track) background to $K_{S}^{0} \rightarrow \pi^{+} \pi^{-}$by removing the immediate vicinity of the event's primary vertex, $3 \mathrm{~mm}$ in $X Y$, from the 1 st (normalization) bin; we call these

\footnotetext{
${ }^{1}$ Note that although the value of the $K_{S}^{0}$ reconstruction efficiency correction in the first bin in $d_{X Y}$ is by definition 1.000, the statistical error on the ratio of the numbers of events in that bin in data and MC translates into an additional, 100\%-correlated uncertainty on all other ratios for the given momentum and polar angle ranges. This is the principal source of the statistical uncertainty on the final value of the mode-dependent $K_{S}^{0}$ efficiency correction.
} 
first 3 millimeters "bin 0". Doing so also has the added benefit of not having to worry too much about the possibility that the imperfect simulation of the random-track background or the potential differences between the $K_{S}^{0}$ quality cut efficiencies (flight-length significance, flight direction) in data and MC may lead to a non-negligible systematic error that is not understood.

\section{C.3 Method}

\section{C.3.1 Data set and selection of $K_{S}^{0} \rightarrow \pi^{+} \pi^{-}$candidates}

We operate with KsDefault $K_{S}^{0}$ candidates $^{2}$ in events that contain at least 5 GoodTracksVeryLoose tracks, two of which are oppositely charged GoodTracksLoose tracks that when combined with the $K_{S}^{0}$ candidate form an object with $m_{\mathrm{ES}}>5.19 \mathrm{GeV} / c^{2}$ and $|\Delta E|<0.3 \mathrm{GeV}$. The vast majority of such events come from the light quark (udsc) continuum, contributions from $\tau^{+} \tau^{-}$production and two-photon processes are small, and only about $1 / 30$ th of the $h^{+} h^{-} K_{S}^{0}$ candidates come from $B$ decays, almost all of them random-track combinations as well.

Since the two ChargedTracks the $K_{S}^{0}$ candidates are composed of are unlikely to also be on the GoodTracksVeryLoose list, there are usually 4 or 5, and never less than 3 non- $K_{S}^{0}$ tracks in the event, which ensures high quality of primary vertex reconstruction. We refit the KsDefault candidate with the constraint that its daughters originate from a

\footnotetext{
${ }^{2}$ I.e., pairs of oppositely charged tracks that have an invariant mass within $25 \mathrm{MeV} / c^{2}$ of the PDG value of $m_{K_{S}^{0}}=497.672 \pm 0.031 \mathrm{MeV} / c^{2}$
} 
Table C.1: Summary of the Release-10 data and SP4 Monte Carlo data sets used in the $K_{S}^{0}$ reconstruction efficiency study. The following values of production cross sections at the $\Upsilon(4 S)$ resonance are assumed: $\sigma(u d s)=2.09 \mathrm{fb}^{-1}, \sigma(c \bar{c})=1.30 \mathrm{fb}^{-1}, \sigma\left(\tau^{+} \tau^{-}\right)=0.94 \mathrm{fb}^{-1}$, $\sigma\left(B^{+} B^{-}\right)=\sigma\left(B^{0} \bar{B}^{0}\right)=1 / 2 \times 1.085 \mathrm{fb}^{-1}$.

\begin{tabular}{|c|c|c|c|c|}
\hline type of events & DCH at 1900 V & DCH at 1960 V & DCH at 1930 V & Total \\
\hline data (on-resonance) & $10.7 \mathrm{fb}^{-1}$ & $9.6 \mathrm{fb}^{-1}$ & $61.6 \mathrm{fb}^{-1}$ & $81.9 \mathrm{fb}^{-1}$ \\
\hline$u \bar{u}, d \bar{d}, s \bar{s} \mathrm{MC}$ & $13.4 \mathrm{fb}^{-1}$ & $16.6 \mathrm{fb}^{-1}$ & $77.4 \mathrm{fb}^{-1}$ & $107.4 \mathrm{fb}^{-1}$ \\
$c \bar{c} \mathrm{MC}$ & $13.4 \mathrm{fb}^{-1}$ & $17.6 \mathrm{fb}^{-1}$ & $76.9 \mathrm{fb}^{-1}$ & $107.9 \mathrm{fb}^{-1}$ \\
$\tau^{+} \tau^{-} \mathrm{MC}$ & $14.7 \mathrm{fb}^{-1}$ & $17.6 \mathrm{fb}^{-1}$ & $81.3 \mathrm{fb}^{-1}$ & $113.6 \mathrm{fb}^{-1}$ \\
$B^{+} B^{-} \mathrm{MC}$ & $18.7 \mathrm{fb}^{-1}$ & $15.6 \mathrm{fb}^{-1}$ & $114.4 \mathrm{fb}^{-1}$ & $148.7 \mathrm{fb}^{-1}$ \\
$B^{0} \bar{B}^{0} \mathrm{MC}$ & $15.0 \mathrm{fb}^{-1}$ & $19.0 \mathrm{fb}^{-1}$ & $115.2 \mathrm{fb}^{-1}$ & $149.2 \mathrm{fb}^{-1}$ \\
\hline
\end{tabular}
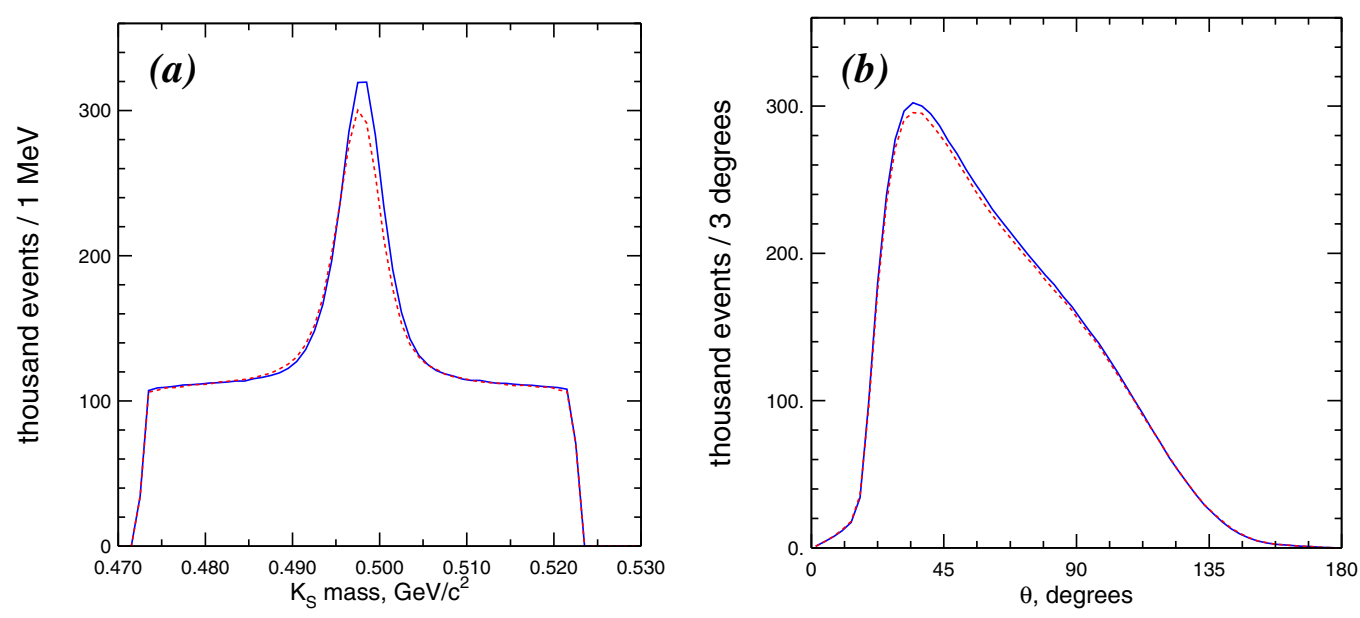

Figure C.1: Distributions of $(a)$ the $\pi^{+} \pi^{-}$invariant mass and $(b)$ the polar angle $\theta_{\text {lab }}$ of the direction from the event's primary vertex to the $K_{S}^{0}$ decay point. Histogram points are connected with a line, dashed for data and solid for Monte Carlo. 

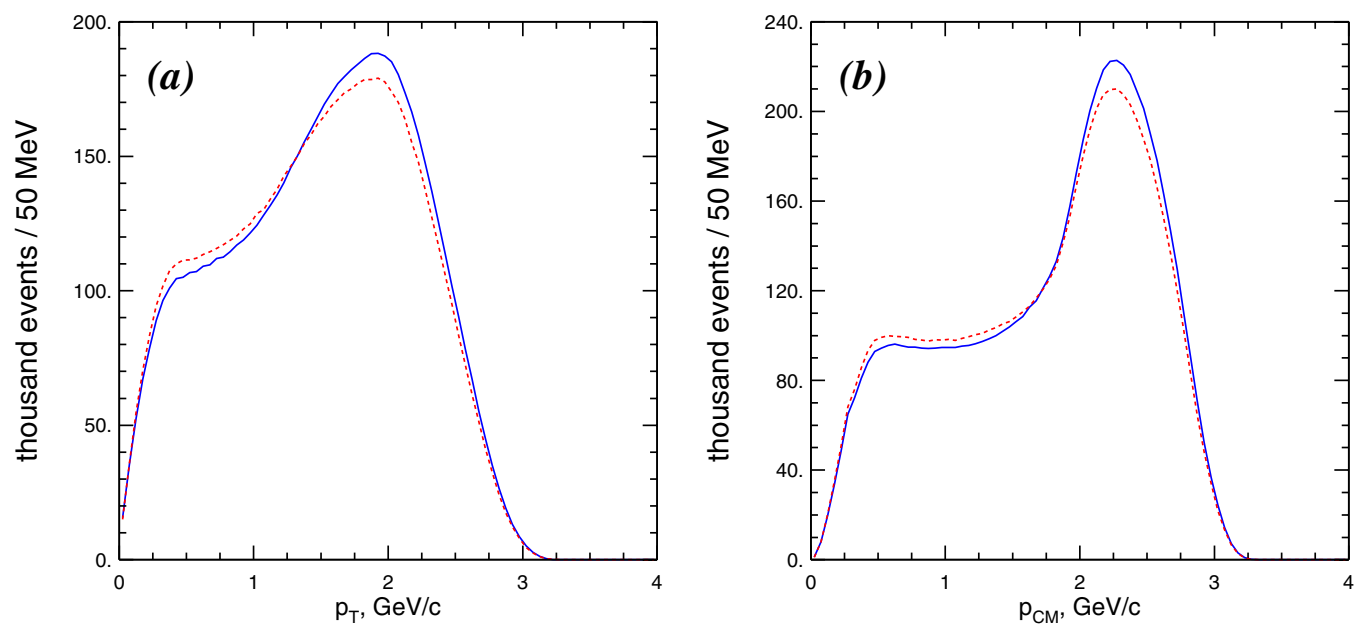

Figure C.2: Distributions of $(a)$ the $K_{S}^{0}$ transverse momentum and $(b)$ the $K_{S}^{0}$ momentum in the $\Upsilon(4 S)$ center-of-mass frame. Histogram points are connected with a line, dashed for data and solid for Monte Carlo. It is evident that the production rate of low-momentum $K_{S}^{0}$ 's is underestimated in SP4 Monte Carlo, while the production rate of high-momentum $K_{S}^{0}$ 's is overestimated.

common vertex (the fit is required not to fail); for true $K_{S}^{0} \rightarrow \pi^{+} \pi^{-}$decays this provides a more precise value of the $\pi^{+} \pi^{-}$invariant mass than the simple 4-vector addition.

The data and MC data sets that are available to us are summarized in Table C.1. Using the numbers in this table we construct a properly weighted data-like combination of MC modes and plot in Figs. C.1, C.2 and C.3 the spectra of several $K_{S}^{0}$-related quantities to give the reader a better understanding of our sample of $K_{S}^{0} \rightarrow \pi^{+} \pi^{-}$decays. Small discrepancies between $K_{S}^{0}$ spectra in data and Monte Carlo are evident, which is one of the reasons why binning the $K_{S}^{0}$ sample into bins of only $d_{X Y}$, as was done before, is not adequate. The finer the binning in angle and momentum, the less the effect of the data/MC $K_{S}^{0}$ spectrum differences; however, one should not choose too fine a binning in order to avoid the difficulty of fitting the $m_{K_{S}^{0}}$ distributions that arise in cases 

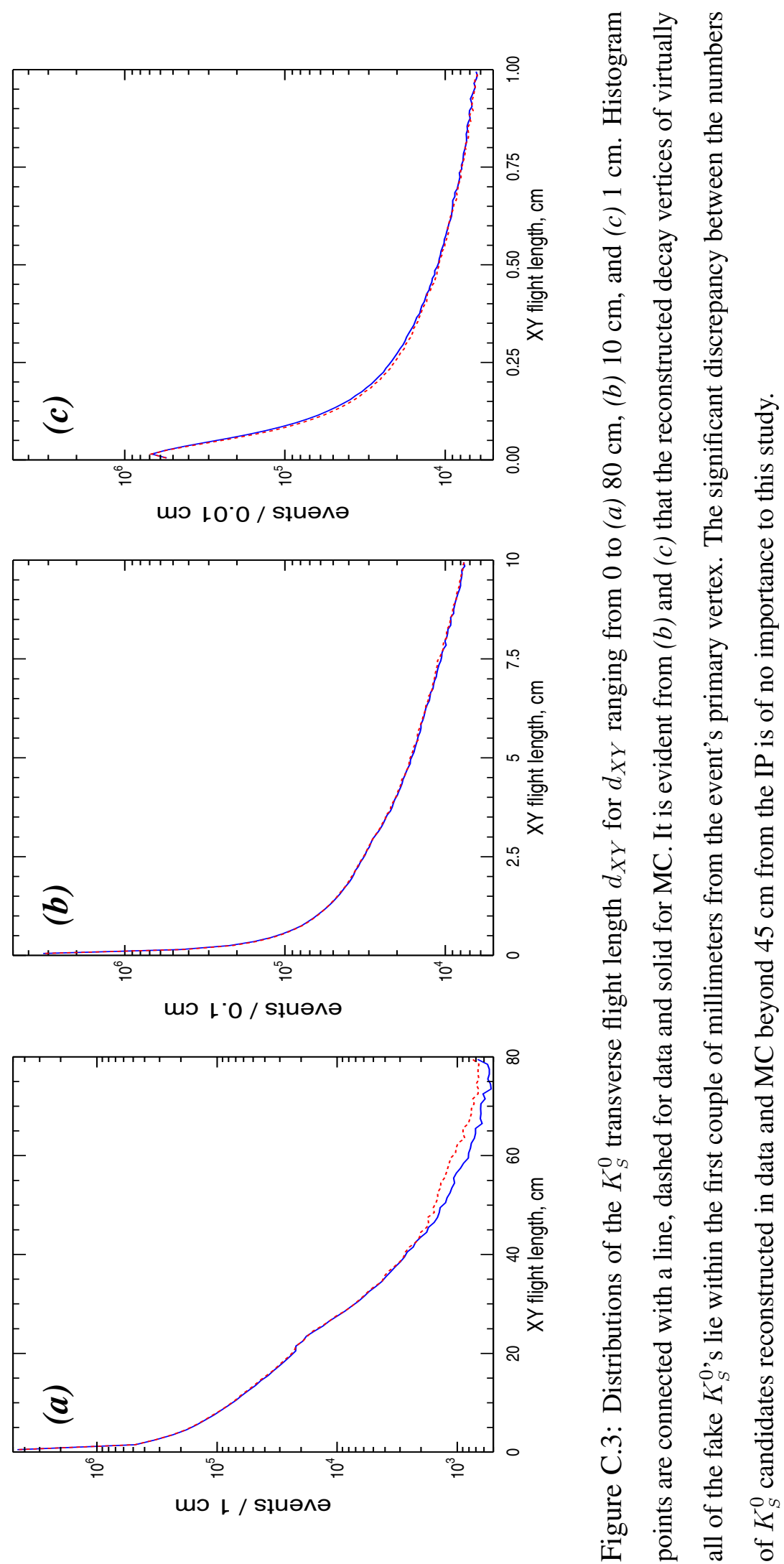
of low statistics.

\section{C.3.2 Binning}

The $K_{S}^{0}$ samples in data and MC are separated into bins of momentum, polar angle and 2-D flight length. In order to verify that the possible difference between the $K_{S}^{0}$ momentum spectra in data and MC is not a significant source of systematic, and also for consistency with other studies, three different binning approaches have been used:

- “Coarse" binning: 4 bins in transverse momentum $p_{T}(0.0-0.4-1.0-2.0-4.0$ $\mathrm{GeV} / c)$ and 4 bins in polar angle $\theta_{\text {lab }}\left(9^{\circ}-46^{\circ}-83^{\circ}-120^{\circ}-157^{\circ}\right)$

- “Alternative" binning: 4 bins in transverse momentum $p_{T}(0.0-0.4-1.0-2.0-4.0$ $\mathrm{GeV} / c)$ and 8 bins in polar angle $\theta_{\text {lab }}\left(7.0^{\circ}-25.6^{\circ}-44.25^{\circ}-62.88^{\circ}-81.5^{\circ}-100.13^{\circ}-\right.$ $\left.118.75^{\circ}-137.38^{\circ}-156.0^{\circ}\right)$. This is the binning that we recommend for use by those who wish to use our tables to compute the data/MC $K_{S}^{0}$ efficiency ratio for their analyses.

- “Fine" binning: 10 bins in center-of-mass momentum $p_{\mathrm{CM}}(0.0-1.0-1.3-1.5-$ 1.7-1.9-2.1-2.3-2.5-2.7-3.5 GeV/c), and 9 bins in polar angle $\theta_{\text {lab }}\left(9^{\circ}-26^{\circ}-36^{\circ}-\right.$ $46^{\circ}-57^{\circ}-69^{\circ}-83^{\circ}-98^{\circ}-120^{\circ}-157^{\circ}$ ). The bin widths vary to reflect the spectrum of $K_{S}^{0}$ 's available to us and to achieve a substantially more uniform bin population that in the case of coarse binning

Common to all binning approaches are the 10 bins in 2-D $(X Y) K_{S}^{0}$ flight length $d_{X Y}$, which is computed as the distance between the primary vertex of the event and 
the refitted $K_{S}^{0}$ decay vertex $(0.0-0.3-1.3-2.78-3.2-4.0-5.4-9.1-11.4-23.6-40.0 \mathrm{~cm})$. This binning roughly reflects the structure of the $B A B A R$ detector (beam pipe at $2.78 \mathrm{~cm}$ from the IP, the five SVT layers at 3.2, 4.0, 5.4, 9.1 and $11.4 \mathrm{~cm}$ from the IP, and the inner radius of the drift chamber at $23.6 \mathrm{~cm}$ from the IP); bins in $d_{X Y}$ are numbered from 0 to 9 , bin 1 being the normalization bin.

Sets of data/MC correction tables are made for each of the three DCH voltages, $1900 \mathrm{~V}$ for data taken from October 15, 1999 to July 14, 2000, $1960 \mathrm{~V}$ for the remainder of year 2000, and $1930 \mathrm{~V}$ since the beginning of year 2001. We also make tables that appropriately combine all DCH voltage ranges in both data and MC. The user of the tables has two choices: to compute corrections separately for each of the three DCH voltage values and weigh them according to the composition of his data set-or to use a combined table (which, of course, is only possible if the user's on-resonance data set is the same as the data set used in this study, see Table C.1). Since corrections computed for the relatively small $1900 \mathrm{~V}$ and $1960 \mathrm{~V}$ data sets are prone to suffer from fluctuations caused by low numbers of events in histograms that we fit, the use of the combined tables is strongly preferred.

For use in systematics studies, and also at the request of several analysis working groups, separate sets of tables are made for several different sets of $K_{S}^{0}$ quality cuts (cuts on the $K_{S}^{0}$ mass, 3-D or $X Y$ flight length or its significance, the 3-D or $X Y$ angle $\alpha$ between the $K_{S}^{0}$ momentum and the line connecting the $K_{S}^{0}$ decay vertex and the primary vertex of the event, and, in one instance, a cut on the $K_{S}^{0} \rightarrow \pi^{+} \pi^{-}$vertexing quality). 


\section{C.3.3 Calculation of the number of $K_{s}^{0}$ 's in each bin}

The $K_{S}^{0}$ mass distributions in each of the bins are fitted with a sum of a double Gaussian and and a constant background ${ }^{3}$. We produce the histograms in PAW and fit them in MN_FIT [105], which provides a substantially more convenient and stable interface to the MINUIT minimization package than PAW does. The binned maximum log likelihood method is used; the default $\chi^{2}$-minimization method is less appropriate for our task as it systematically, in a statistics-dependent way underestimates the number of events in the histogram [87] (see Appendix C.9 for an illustration).

Special measures are taken to ensure without the need for extensive manual checking that in each of the tens of thousands of histograms that we have to fit, the fit results are sensible. Several examples of fitted histograms are shown in Fig. C.4. Here's the procedure that we use:

- The histogram's sideband, $[0.476,0.485] \cup[0.511,0.520] \mathrm{GeV} / c^{2}$, is fitted with a constant, then the area under the constant is subtracted from the sum of histogram's bins in the range $[0.476,0.520] \mathrm{GeV} / c^{2}$ and the statistical error on the difference is computed. We call the ratio of the difference and its error the "a priori" significance of the $K_{S}^{0}$ signal in that histogram. The number of parameters that is subsequently allowed to float in the final fit (with a sum of a double Gaussian and a constant) is determined by the value of this "a priori" significance.

\footnotetext{
${ }^{3}$ The limited statistics in a large fraction of histograms to be fitted makes it impractical to allow the slope of the background to float. Even in cases when the number of events in the histogram is sufficiently large, it is still not necessary to allow a slope in the background because doing so will not, to the first order, change the area of the double Gaussian returned by the fitter.
} 


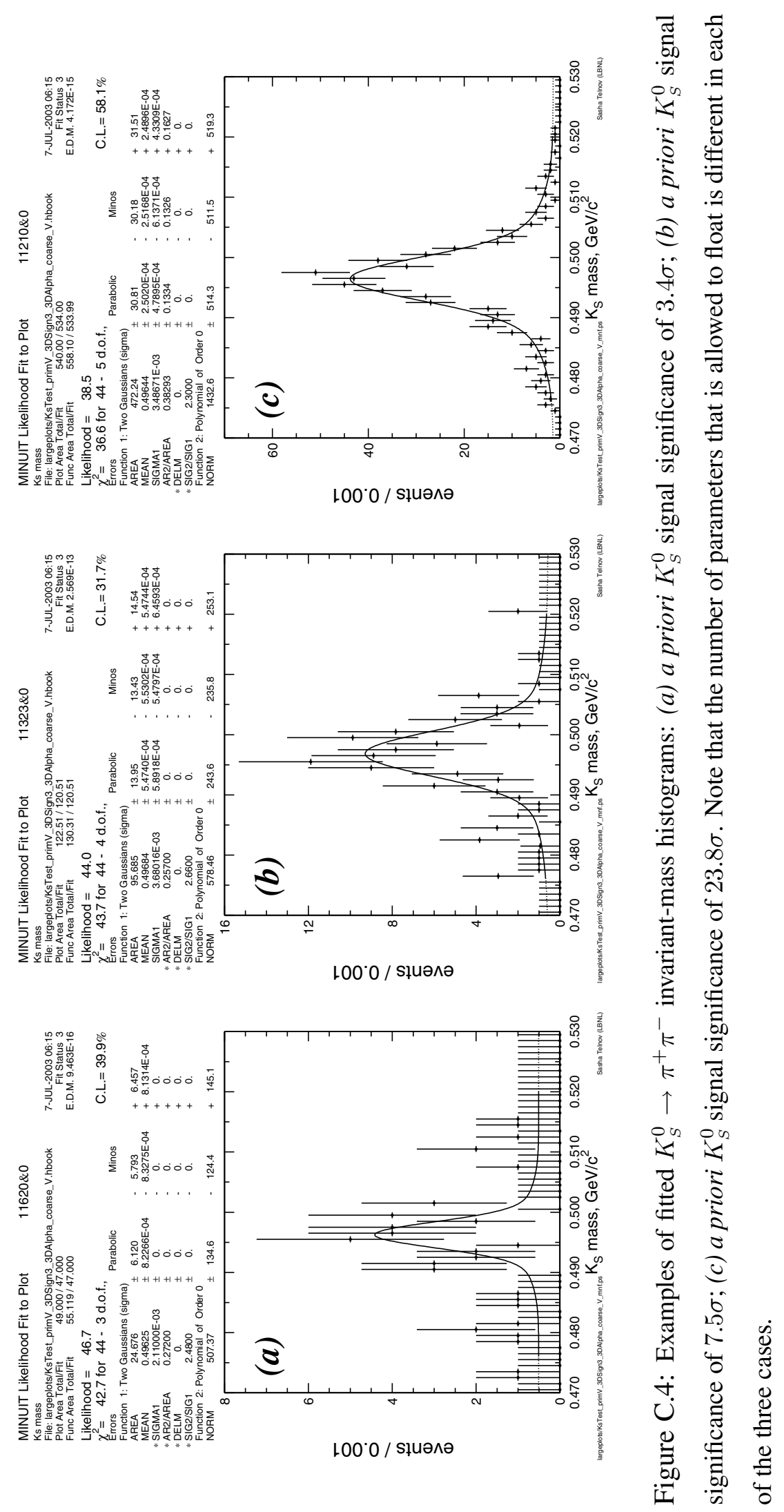


Table C.2: Central values of the $K_{S}^{0}$ mass distributions in Release-10 data and SP4 Monte Carlo as a function of the transverse flight length $d_{X Y}$; the statistical errors are negligible.

\begin{tabular}{|c|c|c|}
\hline transverse flight length & data & MC \\
\hline$d_{X Y}<2.78 \mathrm{~cm}$ & $497.27 \mathrm{MeV} / c^{2}$ & $497.61 \mathrm{MeV} / c^{2}$ \\
$2.78 \mathrm{~cm}<d_{X Y}<3.2 \mathrm{~cm}$ & $497.59 \mathrm{MeV} / c^{2}$ & $497.91 \mathrm{MeV} / c^{2}$ \\
$3.2 \mathrm{~cm}<d_{X Y}$ & $497.85 \mathrm{MeV} / c^{2}$ & $498.10 \mathrm{MeV} / c^{2}$ \\
\hline
\end{tabular}

- "Educated guesses" are made about the starting values of each of the parameters of the final fit:

- The value of the constant from the previous step is used as its starting value, and the estimate of the number of $K_{S}^{0}$ 's mader in the previous step is used as the starting value for the area of the double Gaussian. Both are not allowed to go negative in the fit.

- The mean of the double Gaussian, i.e., the nominal value of the $K_{S}^{0}$ mass, is set to a value determined by binning the $K_{S}^{0}$ samples in data and MC into bins of $d_{X Y}$ only. We observe that the central value of the $K_{S}^{0}$ mass exhibits a jump of $0.5-0.6 \mathrm{MeV} / c^{2}$ as one crosses the PEP-II beam pipe (see Table C.2); this is a well-known tracking effect [104].

- The starting values of the other three parameters, the width of the first Gaussian, the fraction of the total area of the double Gaussian that is due to the second Gaussian, and the ratio of the widths of the second and the first Gaussians, are also determined by making and fitting distributions of $m_{K_{S}^{0}}$ in data 
and $\mathrm{MC}$ in bins of $d_{X Y}$ only; the values for the 0th bin in $d_{X Y}$, where the large amount of combinatoric background prevents us from determining parameters of the double Gaussian with sufficient precision, are assumed to be the same as in the 1 st bin. These starting values are obtained with a $3 \sigma$ cut on the 3-D flight-length significance $\ell / \sigma_{\ell}$ and a cut on the 3-D angle between the $K_{S}^{0}$ momentum and direction of flight $\cos \alpha>0.995$, which are the $K_{S}^{0}$ quality cuts used in our $B^{0} \rightarrow \phi K_{S}^{0}$ branching-fraction analysis [94].

- The mean of the double Gaussian is allowed to float if the "a priori" significance of the $K_{S}^{0}$ signal is greater than $3.0 \sigma$, i.e., nearly always. The difference between the means of the first and the second Gaussians is always constrained to zero.

- The width of the first Gaussian (and so the tied to it width of the second Gaussian) is allowed to float if the "a priori" significance of the $K_{S}^{0}$ signal is greater than $6.0 \sigma$. The exception from this are the high-background 0th and 1 st bins in $d_{X Y}$ in the case when no $K_{S}^{0}$ quality cuts are used, in which case the cut-off for the 0th bin is $8.0 \sigma$ for the "fine" binning, $10.0 \sigma$ for the "alternative" binning, and $12.0 \sigma$ for the "coarse" binning; for the 1st bin the cut-off is $8.0 \sigma$. It is also $8.0 \sigma$ in the 0th and 1st bins in $d_{X Y}$ when no $\cos \alpha$ cut is used. The fraction of the area that is due to the second Gaussian is allowed to float if it is greater than $20.0 \sigma$. The ratio of the second and the first Gaussian's width is always fixed-which, given the not-so-great numbers of events in even the highest-statistics histograms and a reasonable choice of its value, does not prevent us from obtaining good-quality 
fits.

The results of this fitting procedure are written out to a file with a filename descriptive of the set of $K_{S}^{0}$ quality cuts used in its preparation and with the extension . log. The file's format is five integers: bin numbers for momentum, polar angle, transverse flight length, DCH voltage $(1=1900 \mathrm{~V}, 2=1960 \mathrm{~V}, 3=1930 \mathrm{~V}, 0=$ combined $)$, and event type $(0=$ data, $3=\mathrm{MC})$, and seven floating-point numbers: the area of the double Gaussian (i.e., the number of $K_{S}^{0}$ 's) and the error on the area of the double Gaussian, followed by the three numbers that can be used by the users of our tables to analycally compute the efficiency of their $m_{K_{S}^{0}}$ cuts (the width of the first Gaussian in $\mathrm{MeV} / \mathrm{c}^{2}$, the fraction of the total area that is due to the second Gaussian, and the width of the second Gaussian in $\mathrm{MeV} / \mathrm{c}^{2}$ ), followed by the efficiency of a $12 \mathrm{MeV} / c^{2} m_{K_{S}^{0}}$ cut [94] that we compute by numerical integration; last and for purely informational purposes comes the value of the "a priori" significance of the $K_{S}^{0}$ signal in this bin.

\section{C.3.4 Calculation of the efficiency table elements}

In the next step, done in a simple FORTRAN program, we read the .log file described in the previous section and produce two . dat files with normalized ratios of $K_{S}^{0}$ yields (i.e., the correction factors that the end user will be applying to his signal MC sample) and their uncertainties; the first concerns itself only with the ratio of $K_{S}^{0}$ reconstruction efficiencies in data and MC, while the second also takes into account the bin-by-bin variations of the $m_{K_{S}^{0}}$ resolution in data and MC and depends on the specific value of 
the $K_{S}^{0}$ mass cut used by the analyst.

We define the values of the normalized ratios $R_{i j k}$ and their (absolute) uncertainties $\sigma_{R_{i j k}}$, where the third index $k$ stands for the number of the $d_{X Y}$ bin, to be

$$
R_{i j k}=\left(\frac{N_{i j k}}{M_{i j k}}\right) /\left(\frac{N_{i j 1}}{M_{i j 1}}\right),
$$

$$
\begin{aligned}
\sigma_{R_{i j k}} & =R_{i j k} \sqrt{\left(\frac{\sigma_{N_{i j k}}}{M_{i j k}}\right)^{2}+\left(\frac{N_{i j k} \sigma_{M_{i j k}}}{M_{i j k}^{2}}\right)^{2}} /\left(\frac{N_{i j k}}{M_{i j k}}\right) \\
& =\sqrt{\left(\frac{\sigma_{N_{i j k}}}{M_{i j k}}\right)^{2}+\left(\frac{N_{i j k} \sigma_{M_{i j k}}}{M_{i j k}^{2}}\right)^{2}} /\left(\frac{N_{i j 1}}{M_{i j 1}}\right)
\end{aligned}
$$

where $N_{i j k}$ and $\sigma_{N_{i j k}}$ are the numbers of $K_{S}^{0}$ 's and their uncertainties in data and $M_{i j k}$ and $\sigma_{M_{i j k}}$ are the numbers of $K_{S}^{0}$ 's and their uncertainties in MC. By construction $R_{i j 1}=$ 1.

For the tables that take into account the $K_{S}^{0}$ mass resolutions in data and $\mathrm{MC}$, we perform numerical integration to determine the efficiencies of the $\left|m_{\pi+\pi-}-m_{K_{S}^{0}}\right|<$ $12 \mathrm{MeV} / c^{2}$ cut in data $\left(\epsilon_{i j k}\right)$ and $\mathrm{MC}\left(\varepsilon_{i j k}\right)$. Then the $K_{S}^{0}$ efficiency correction table elements $R_{i j k}^{\text {eff }}$ and their respective uncertainties $\sigma_{R_{i j k}^{\text {eff }}}$ are given by

$$
R_{i j k}^{\mathrm{eff}}=R_{i j k} \frac{\epsilon_{i j k}}{\varepsilon_{i j k}}
$$


and

$$
\sigma_{R_{i j k}^{\mathrm{eff}}}=\sigma_{R_{i j k}} \frac{\epsilon_{i j k}}{\varepsilon_{i j k}}
$$

\section{C.3.5 Treatment of special cases}

As was already mentioned in Section C.3.3, the process of computing data/MC correction factors for tens of thousands of bins needs to be automated to handle in a graceful and sensible manner the pathological cases that may result from fitting histograms with poor statistics. In addition to the measures discussed in Section C.3.3, the following "sanity checks" are used:

- In a few cases MINUIT returns an abnormally small parabolic error on the area of the double Gaussian, i.e., the number of $K_{S}^{0}$ candidates. We set to 3.0 the values of $\sigma_{M_{i j k}}$ and $\sigma_{N_{i j k}}$ that are less than 3.0.

- If either $M_{i j k}<3.0$ or $N_{i j k}<3.0$, we set $R_{i j k}=1.0 \pm 0.5$.

- If $R_{i j k}$ falls outside the $[0.7,1.3]$ range and $\sigma_{R_{i j k}}>0.5$, we set $R_{i j k}$ either to 0.7 or to 1.3 .

- If $\sigma_{R_{i j k}}>0.5$, we set $\sigma_{R_{i j k}}=0.5$. 
- If, as a result of these steps, $\sigma_{R_{i j k}}=0.5$, we set $R_{i j k}^{\text {eff }}=R_{i j k}(1.0,0.7$ or 1.3$)$ and $\sigma_{R_{i j k}^{\text {eff }}}=0.5$

Only a small fraction of bins have these "sanity checks" used on them, most of them by construction: e.g., there are no $K_{S}^{0}$ candidates with $p_{T}>2.0 \mathrm{GeV}$ and $\theta_{\text {lab }}>120^{\circ}$, etc. The increase of the statistical error $\sigma_{\mathcal{R}}$ on the value of the overall mode-dependent correction factor $\mathcal{R}$ that arises from the conservative choice $( \pm 0.5)$ of $\sigma_{R_{i j k}}$ 's in lowstatistics bins is negligible.

\section{C.4 Determination of the mode-dependent data/MC over- all efficiency ratio}

Let $H_{i j k}$ be the number of events in the signal MC sample that falls within the bin $(i j k)$. The relative weight of a $K_{S}^{0}$ efficiency correction table element $R_{i j k}$ is $H_{i j k} / H_{\text {tot }}$, where $H_{\text {tot }}=\sum H_{i j k}$, and its statistical uncertainty is $\sqrt{H_{i j k}} / H_{\text {tot }}$.

The central value of the overall mode-dependent data/MC efficiency ratio is simply

$$
\mathcal{R}=\frac{1}{H_{\text {tot }}} \sum_{i j k} H_{i j k} R_{i j k}
$$

Calculation of the statistical uncertainty on this number is slightly non-trivial since we have to take into account the fact that the statistical uncertainty on the normalization bin ratio, $\sigma_{R_{i j 1}}$, influences the entire row $(i j)$. Substituting $R_{i j k}$ in Eq. C.5 with the 
expression in Eq. C.1 and differentiating the resulting expression with respect to each of the variables that enter it while remembering that $R_{i j 1}=1$, we obtain

$$
\sigma_{\mathcal{R}}=\frac{1}{H_{\mathrm{tot}}} \sqrt{\sum_{i j}\left(\sum_{k} H_{i j k} R_{i j k}^{2}+\sum_{k \neq 1}\left(H_{i j k} \sigma_{R_{i j k}}\right)^{2}+\sigma_{R_{i j 1}}^{2}\left(\sum_{k \neq 1} H_{i j k} R_{i j k}\right)^{2}\right)}
$$

where the first term reflects the finite size of the signal MC sample used in the study and is generally the smallest, the second term reflects the statistical uncertainties on the number of $K_{S}^{0}$ 's in bins other than the normalization bin, and the third term, the dominant one, reflects the dependence on the statistical precision of $R_{i j 1}$ of the correction factors in each of the bins $R_{i j k}, k \neq 1$.

The values of $R_{i j k}$ and $\sigma_{R_{i j k}}$ are provided to the end user in a . dat file made with the set of $K_{S}^{0}$ selection cuts most similar to that used in the user's analysis. The user should provide the numbers $H_{i j k}$ that represent the $K_{S}^{0}$ spectrum in the mode under consideration. The user should also either write the code that sources the .dat file and implements Eq. C.5 and Eq. C.6 or adopt the FORTRAN implementation of such code provided by the Author.

The values of efficiency ratios $R_{k}$ and their uncertainties $\sigma_{R_{k}}$ in each of the bins in $d_{X Y}$ (as in Fig. C.5) are

$$
R_{k}=\sum_{i j} H_{i j k} R_{i j k} / \sum_{i j} H_{i j k}
$$


and

$$
\sigma_{R_{k}}=\sqrt{\sum_{i j}\left(H_{i j k} R_{i j k}^{2}+\left(H_{i j k} \sigma_{R_{i j k}}\right)^{2}\right)} / \sum_{i j} H_{i j k} .
$$

\section{C.5 Test modes and systematics studies}

We use large samples of $B^{0} \rightarrow \phi K_{S}^{0}$ and $B^{0} \rightarrow \pi^{+} D^{-}\left(D^{-} \rightarrow K_{S}^{0} \pi^{-}\right)$signal Monte Carlo, the first of which possesses a nearly monochromatic $K_{S}^{0}$ spectrum in the PEP-II center-of-mass frame, which is relevant to several quasi-two-body charmless and charmonium modes studied at BABAR, while the second has a $K_{S}^{0}$ spectrum similar to that in $B \rightarrow \phi K^{*}$ or $B \rightarrow \rho K^{*}\left(K^{*} \rightarrow \pi K_{S}^{0}\right)$. The results are provided in Tables C.3, C.4 and C.5, which are located at the end of this note. The quoted errors are statistical, computed according to Eq. C.6. The spread of central values as a function of the set of cuts used in the making of the efficiency correction tables is a measure of the systematic error. The central value of the overall correction in $B^{0} \rightarrow \phi K_{S}^{0}$ is lower than in $B^{0} \rightarrow \pi^{+} D^{-}\left(D^{-} \rightarrow K_{S}^{0} \pi^{-}\right)$because the average momentum of $K_{S}^{0}$ 's in $B^{0} \rightarrow \phi K_{S}^{0}$ is significantly higher, causing a greater number of them to decay outside the SVT, where SP4 MC systematically overestimates the $K_{S}^{0}$ reconstruction efficiency (see Fig. C.5). 


\section{C.6 Results}

For the decay mode $B^{0} \rightarrow \phi K_{S}^{0}$, with the nominal cuts used in our analysis (3-D $3 \sigma$ on the flight-length significance and 3-D $\cos \alpha>0.995$ [94]; "coarse" tables are being used) we find that the data/MC $K_{S}^{0}$ reconstruction efficiency ratio is $0.948 \pm 0.020$ for $1900 \mathrm{~V}$ runs, $0.998 \pm 0.021$ for $1960 \mathrm{~V}$ runs, and $0.977 \pm 0.010$ for $1930 \mathrm{~V}$ runs; the overall data/MC efficiency ratio is $0.974 \pm 0.010$ (Fig. C.5a).

On top of this, the $K_{S}^{0}$ mass resolution is worse in data than in MC, especially for the $K_{S}^{0}$ 's decaying outside the SVT, and more so the lower the DCV voltage. The effect of the $12 \mathrm{MeV} / c^{2} K_{S}^{0}$ mass cut is to bring the efficiency ratios down to $0.937 \pm 0.019$ for $1900 \mathrm{~V}$ runs, $0.991 \pm 0.021$ for $1960 \mathrm{~V}$ runs, and $0.967 \pm 0.010$ for $1930 \mathrm{~V}$ runs; the overall efficiency ratio is $0.964 \pm 0.010$ (Fig. C.5b).

Looking at the value of $\mathcal{R}$ as a function of $d_{X Y}$ (Fig. C.5), it is clearly seen that the difference in the values of $\mathcal{R}$ for different DCH voltages is mostly due to the 8 th and the 9th bins in $d_{X Y}$, where tracking is provided solely by the DCH. It is expected that the DCH efficiency should be lower at lower voltages; it is somewhat surprising that the effect is so large and that it is not properly described in Monte Carlo.

Similarly, for $B^{0} \rightarrow \pi^{+} D^{-}\left(D^{-} \rightarrow K_{S}^{0} \pi^{-}\right)$(with a 3-D $5 \sigma$ cut on the flight-length significance and 3-D $\cos \alpha>0.995$ [97]), the $K_{S}^{0}$ reconstruction efficiency ratios determined with the "coarse" tables are $0.961 \pm 0.011$ for $1900 \mathrm{~V}, 1.002 \pm 0.012$ for $1960 \mathrm{~V}$, and $0.983 \pm 0.007$ for $1930 \mathrm{~V}$; the overall efficiency ratio is $0.982 \pm 0.006$ (Fig. C.6a).

On top of this, the effect of the $12 \mathrm{MeV} / c^{2} K_{S}^{0}$ mass cut is to bring the efficiency 


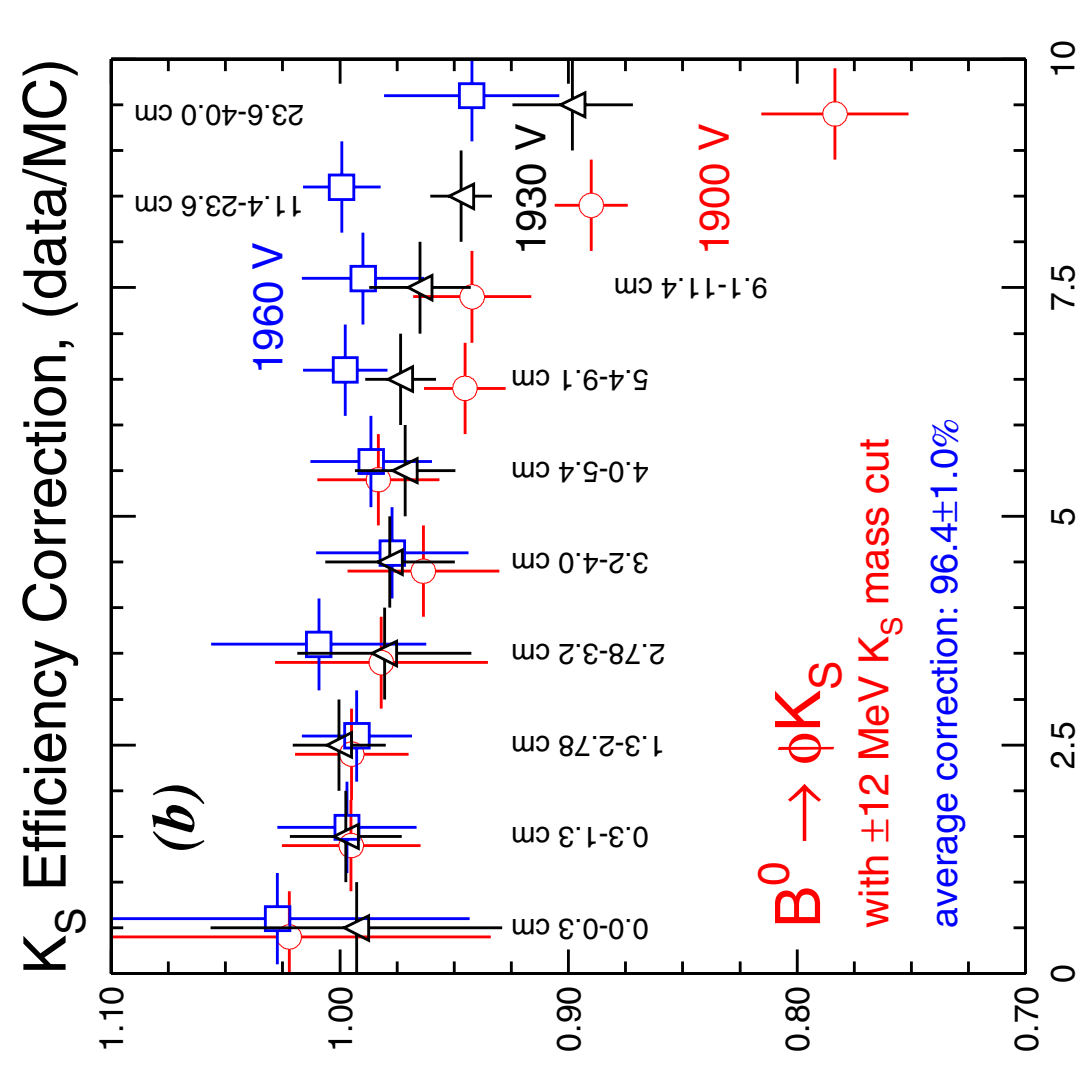

ב

$\frac{1}{2}$

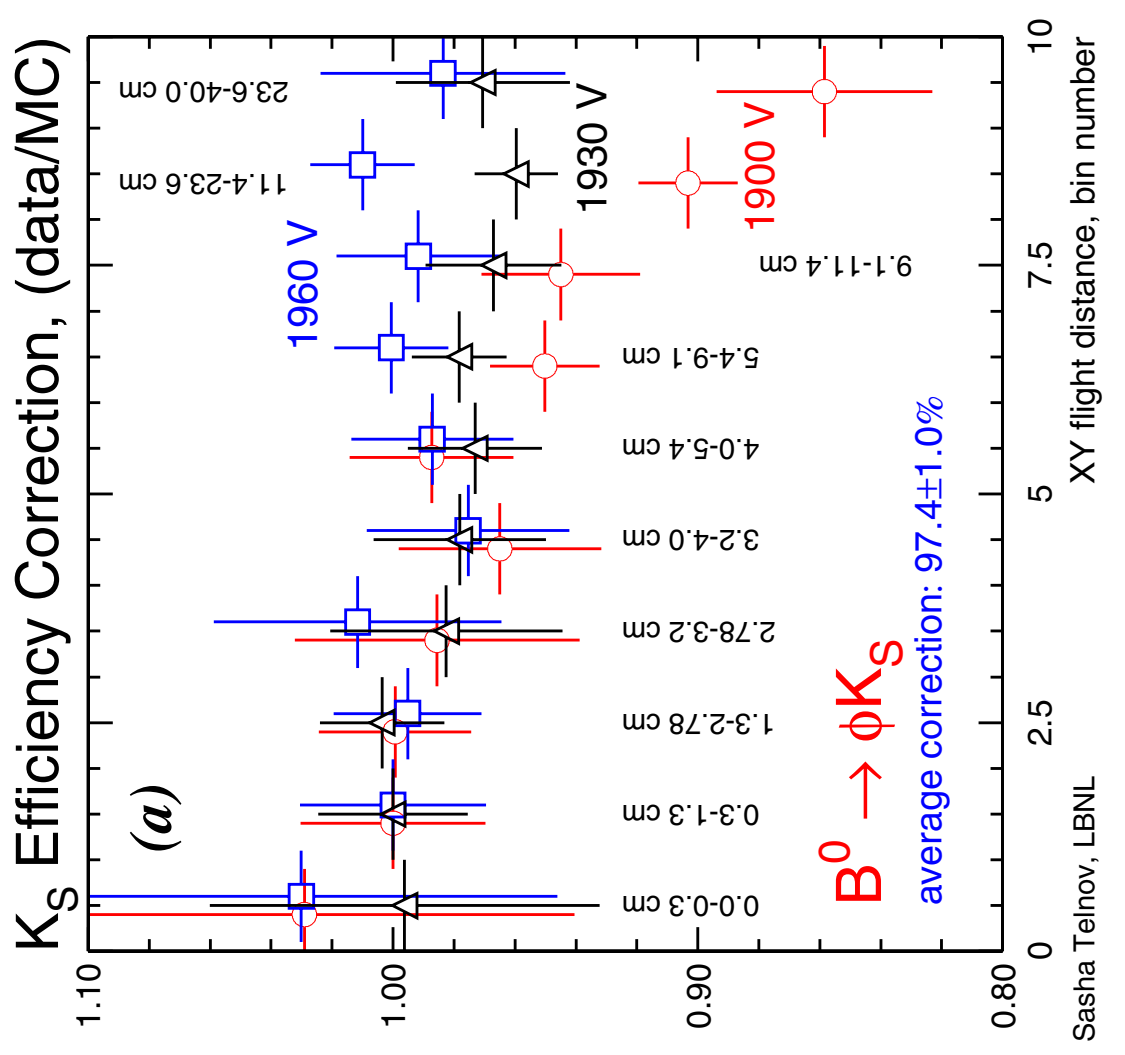

运

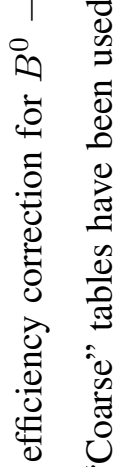

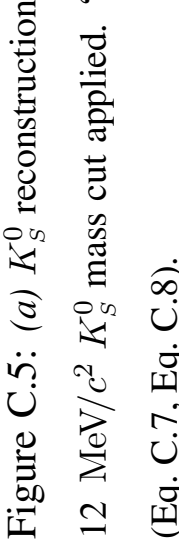


ratios for $B^{0} \rightarrow \pi^{+} D^{-}\left(D^{-} \rightarrow K_{S}^{0} \pi^{-}\right)$down to $0.955 \pm 0.011$ for $1900 \mathrm{~V}$ runs, $0.998 \pm$ 0.012 for $1960 \mathrm{~V}$ runs, and $0.978 \pm 0.007$ for $1930 \mathrm{~V}$ runs; the overall efficiency ratio is $0.977 \pm 0.006$ (Fig. C.6b).

The fact that the value of the correction is slightly larger for $B^{0} \rightarrow \pi^{+} D^{-}\left(D^{-} \rightarrow\right.$ $\left.K_{S}^{0} \pi^{-}\right)$that for $B^{0} \rightarrow \phi K_{S}^{0}$ is to be expected: the spectrum of $K_{S}^{0}$ 's in $B^{0} \rightarrow \phi K_{S}^{0}$ is harder than that in $B^{0} \rightarrow \pi^{+} D^{-}\left(D^{-} \rightarrow K_{S}^{0} \pi^{-}\right)$, and so a larger fraction of $K_{S}^{0}$ s decays outside the SVT, where Monte Carlo overestimates both the reconstruction efficiency and the precision with which the two-pion invariant mass in $K_{S}^{0} \rightarrow \pi^{+} \pi^{-}$is reconstructed.

A cross-comparison of the results in Tables C.3, C.4 and C.5 reveals that as long as one applies a sensible $\cos \alpha$ cut $(\cos \alpha>0$ does not count as such), the spread of central values is less than $0.5 \%$ for all choices of binning and cuts that have been tried. The effect from the possible contamination of the $K_{S}^{0}$ sample with $\Lambda \rightarrow p \pi^{-}$decays is negligible. Varying the boundaries of the normalization bin changes the central value by a negligible amount as well.

This, however, does not mean that the systematic error is under $0.5 \%$ as long as one applies a $\cos \alpha$ cut: the fact that the tracking efficiencies in data and MC are equal within the normalization bin has been established with no additional cuts on track quality; the efficiencies of the cuts on the flight length and the angle $\alpha$ may be different in data ana MC. Once we include results obtained with no $\cos \alpha$ cut and/or no flight length cut, the spread of central values reaches the $\lesssim 1.5 \%$ level.

Combined with the statistical error, this leads to a $\sim 2.0 \%$ total uncertainty on the 


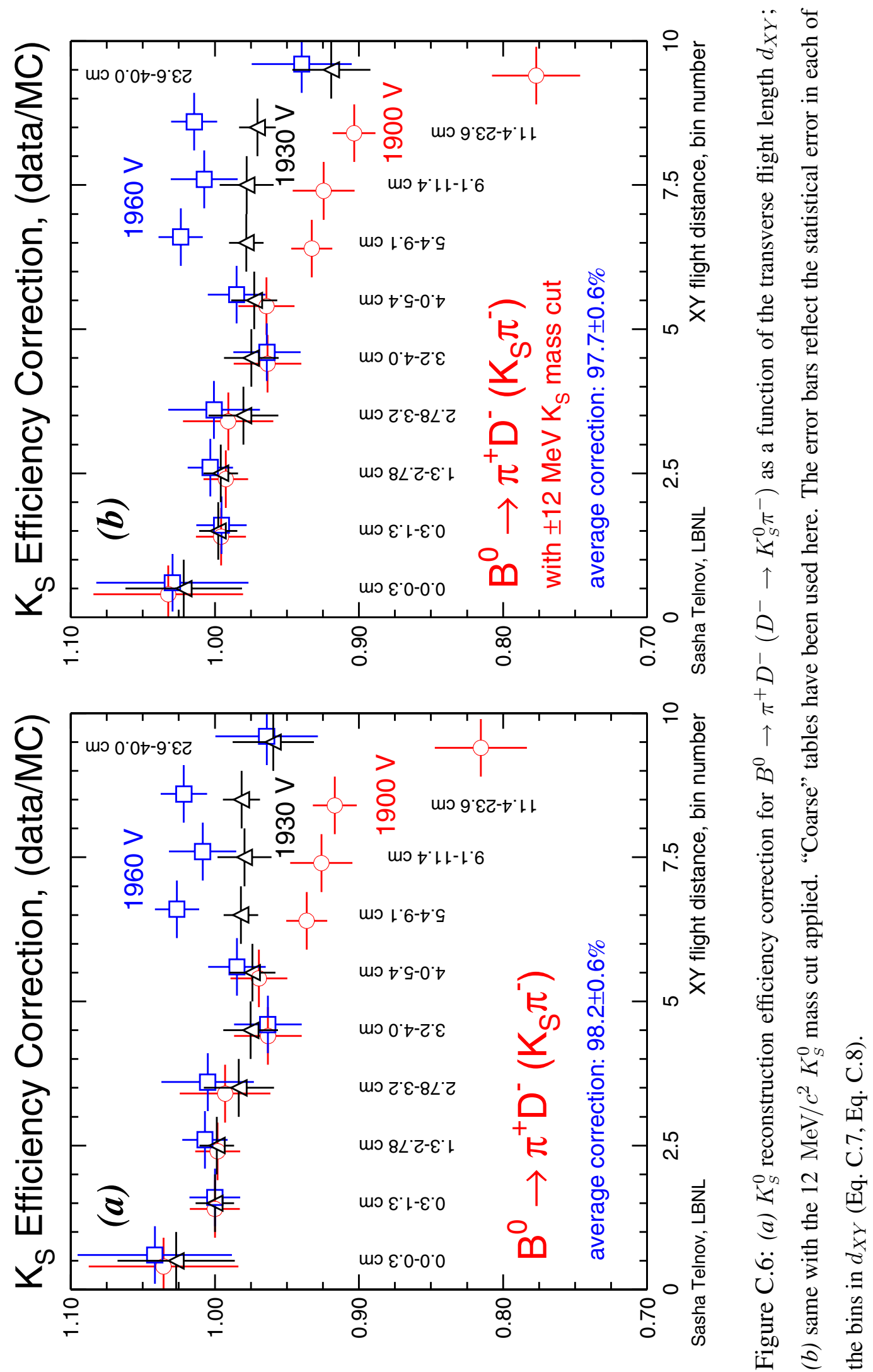


$K_{S}^{0}$ reconstruction efficiency correction that one obtains with our tables. One, perhaps, could try to take a more optimistic approach to interpreting the results shown in Tables C.3, C.4 and C.5 and arrive to a value as low as $1.0 \%-1.2 \%$. We, however, believe that such an attempt would be unwise unless the undertaker is willing to invest a substantial amount of time and effort in proving his or her point.

\section{C.7 Summary}

For the modes $B^{0} \rightarrow \phi K_{S}^{0}$ and $B^{0} \rightarrow \pi^{+} D^{-}\left(D^{-} \rightarrow K_{S}^{0} \pi^{-}\right)$, which are representative of most cases of $K_{S}^{0}$ use in $B A B A R$ analyses, we are able to determine the data/MC $K_{S}^{0}$ reconstruction efficiency correction with a statistical error of 0.6-1.0\%. Systematics studies indicate that the systematic uncertainty is $\lesssim 1.5 \%$, which leads to a $\sim 2.0 \%$ total uncertainty on the $K_{S}^{0}$ reconstruction efficiency correction that one obtains with our tables. Since no BABAR analysis (with the notable exception of $B^{0} \rightarrow \psi K_{S}^{0}$ ) would benefit from knowing the value of the data/MC $K_{S}^{0}$ reconstruction efficiency correction with better than $\sim 3 \%$ precision, our study has achieved its goal.

\section{C.8 The Recipe}

\section{C.8.1 Files and Tools}

The home directory of our $K_{S}^{0}$ efficiency correction tables is located on SLAC NFS:

/nfs/farm/babar/calib/TrackEff/CorrectionTables/KSeff/R10/BAD677/ 


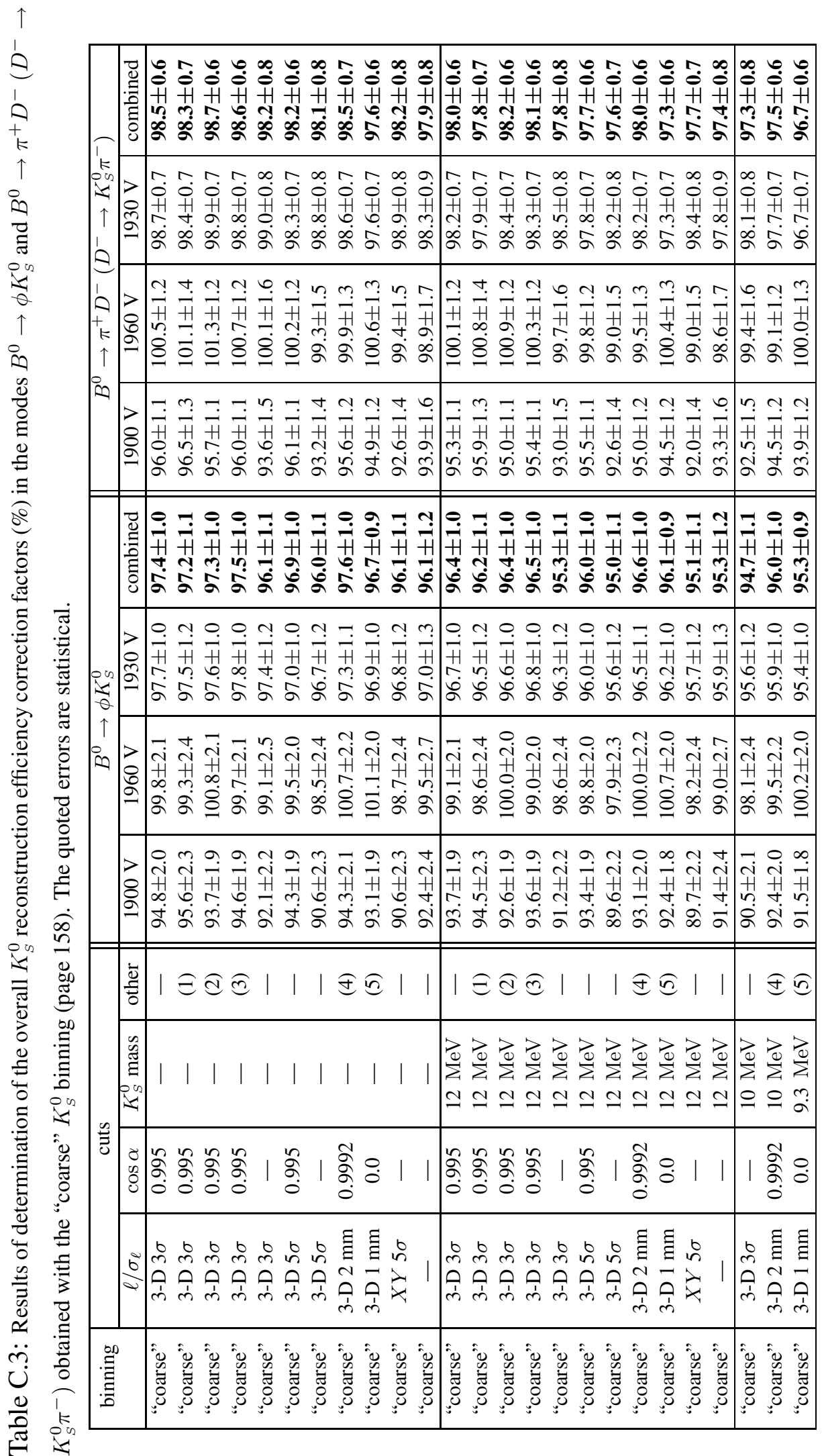

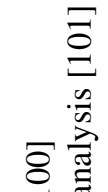

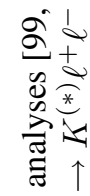

류 윯

해음

예

प्र

공

苍总

강

践

莺

हี

ल क क्ष

ๆ? ๆ

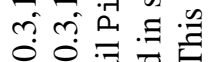

으유

즐

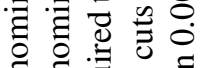

马

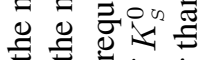

पै

च च

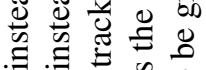

घ 1 9

हैำ

ले

을요

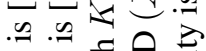

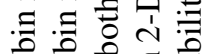

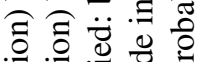

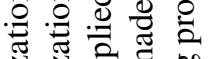

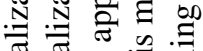

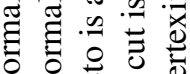

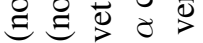

क满

巳巳

정 त्र 


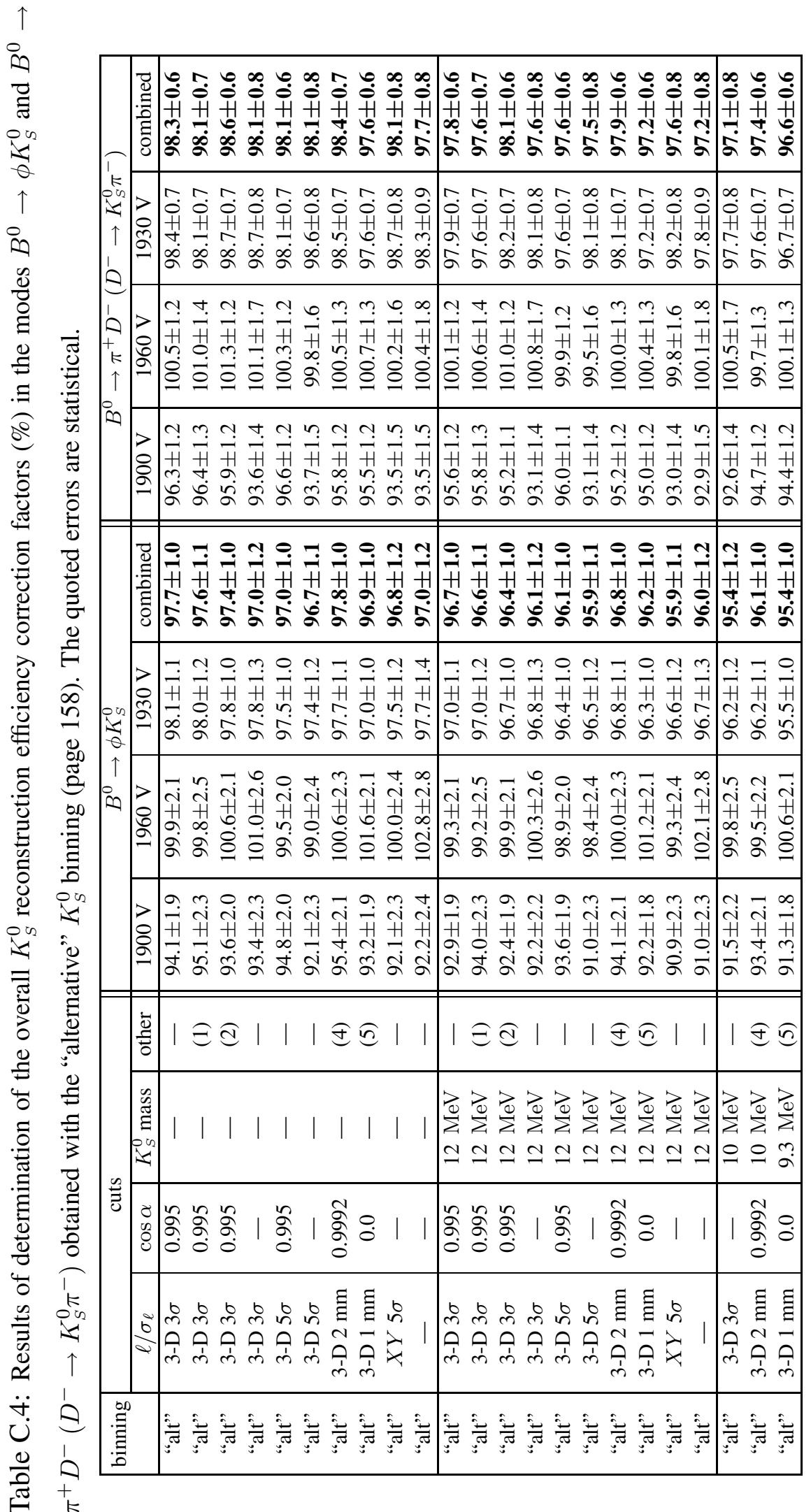

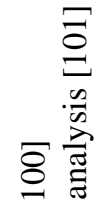

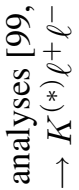

유

흉 옹

U

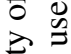

氙

录过

\&

घ $\approx$ 至

๗๘苛

ले ले

007

ㅋㅡㅋㅀㅇ

킁 节

$\exists$ \% 的

武的

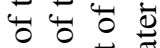

च च

:

콩

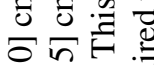

लिंड

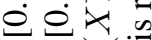

.

ㅋํำ

สิ ิิ)

记 芯

크 브

है 吾

$\Xi \Xi \varnothing$

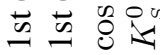

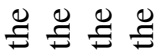

저요 
All file and directory names mentioned in the Appendix are relative to this directory unless noted otherwise.

\section{The ntuples}

The complete set of HBOOK ntuples used in our study, $906 \mathrm{MB}$ in size, in archived on tape in the SLAC HPSS system. To retrieve it from tape, issue the command mstore get/nfs/mstore/u/avtelnov/Physics/KsEffR10/ntuples_Ksphi_KsTest_ 20030706.tar

from any general-use Solaris or Linux system at SLAC, wait until the file becomes available at the specified NFS location, copy the file to a temporary location and untar it into a directory named merged/. Filenames are self-explanatory, e.g. the file named

mgen_SP4_uds_Ksphi_KsTest_1960V.hbook

contains information on $K_{S}^{0}$ candidates in SP4 generic $u d s$ MC made for DCH HV set to $1960 \mathrm{~V}$. The record length in these files is 4096 words, not the default 1024 - so, in PAW one would open these files with a command such as

h/file 3 mgen_SP4_uds_Ksphi_KsTest_1960V.hbook 4096

The ntuple ID is 12 , it contains 14 variables:

- trk3labP: $K_{S}^{0}$ candidate's laboratory-frame momentum, in $\mathrm{GeV} / c$;

- tr3laThe: $K_{S}^{0}$ candidate's laboratory-frame theta, in radians;

- trk3cmsP: $K_{S}^{0}$ candidate's center-of-mass momentum, in $\mathrm{GeV} / c$; 
- K3mass: $K_{S}^{0}$ candidate's refitted mass, in $\mathrm{GeV} / c^{2}$;

- K3prob: $K_{S}^{0}$ candidate's vertexing probability $(\in[0.0,1.0])$;

- vK3dist: $K_{S}^{0}$ candidate's 3-D flight length w.r.t. the event's primary vertex, in $\mathrm{cm}$

- vK3distE: the error on the above quantity, in cm;

- vK3diXY, vK3diXYE: same, but for the $X Y$ flight length;

- vK3alfa: 3-D $\alpha$, in radians;

- vK3alXY: $X Y \alpha$, in radians;

- GTVLLen: number of GTVL tracks in this event;

- runNum: run number;

- protVeto: $=1$ if any of the $K_{S}^{0} \rightarrow \pi^{+} \pi^{-}$tracks passes the veryTight ML proton selector, $=0$ otherwise.

\section{The histograms}

The kumacs/ directory contains the PAW macros used to make the histograms of $K_{S}^{0}$ mass distributions in each bin. The file names reflect the set of $K_{S}^{0}$ quality cuts used, either explicitly or by the name of the institution responsible for the analysis that uses that particular set of cuts. These macro files are pretty well documented; look inside for bin and histogram ID definitions and other information. The histograms produced by these macros reside in the hbooks / directory; they have been bzip2'ed to save space. 
We used MN_FIT [105] version 4.07/32 to fit these histograms. If you are on a Solaris machine at SLAC, you can access this version of MN_FIT by typing

setenv MN_FIT /afs/slac.stanford.edu/u/ec/avtelnov/VOL2/mn_fit_40732

mn_fit

The MN_FIT macro files are stored in the directory mnfs/. The Postscript files produced by these scripts have been bzip2'ed and copied to ps/. Beware: these .ps files are huge!

\section{The tables}

The .log files produced by MN_FIT are stored in tables/logs/; their format is described in Section C.3.3. You will need these files only if you wish to use the per-bin $K_{S}^{0}$ mass resolution information contained in these files to compute the efficiency of your $m_{K_{S}^{0}}$ cut $^{4}$.

The dat table files are produced from the .log files with the FORTRAN code that can be found in tools/log2dat/. The tables themselves are kept in tables/: the files made with no $m_{K_{S}^{0}}$ cut are in tables/noMassCut/, and the files made with a $m_{K_{S}^{0}}$ cut are in tables/withMassCut/. Of the tables made with an $m_{K_{S}^{0}}$ cut, all but those with _Colo or _UCSB (and no _12MeV) in their file name are made with a $12 \mathrm{MeV} / \mathrm{c}^{2}$ cut. Please refer to Section C.3.4 for the description of the . dat table files' format.

\footnotetext{
${ }^{4}$ Colorado and UCSB/Caltech folks: $m_{K_{S}^{0}}$ cut efficiencies have already been computed for your analyses, see Tables C.3, C.4 and C.5.
} 


\section{Other tools}

Instead of writing your own code to implement the formulae in Section C.4, you may want to reuse the FORTRAN code developed by the Author. Please see the files .F in tools/computeR/. Documentation is within the source code.

\section{C.8.2 Give me the recipe already!}

If you have read the note and the "Files and Tools" section above, you should already have a reasonably clear idea how to proceed. Here's an "executive summary" for the impatient:

First, ask yourself: "what is the systematic error on the $K_{S}^{0}$ reconstruction efficiency that I can live with?" If you are in the process of discovering a new decay mode, there is surely no need to know it with a better than $3 \%$ accuracy. Just take $98.0 \pm 3.0 \%$ and go home.

If you have ten minutes to spare, go to Table C. 4 and find in the "cuts" column the set of $K_{S}^{0}$ selection cuts that most closely resembles yours. Then take the corresponding number in bold from the " $B^{0} \rightarrow \phi K_{S}^{0}$ combined" column if the $K_{S}^{0}$ 's in your process are predominantly high-momentum (around $2 \mathrm{GeV} / c$ ) in the $\Upsilon(4 S)$ center-of-mass; take the number from the " $B^{0} \rightarrow \pi^{+} D^{-}\left(D^{-} \rightarrow K_{S}^{0} \pi^{-}\right)$combined" column otherwise. Take this as your central value and use $3.0 \%$ as the systematic error.

If, however, you wish to get the systematic error down to the $2.0 \%$ level (or perhaps, at your own risk, to an even lower value), you actually have to read this note to under- 
stand what is going on, pick the most appropriate efficiency correction table and work out a way to incorporate it into your code-probably the same code that you use with tracking and PID tables. Good luck!

\section{C.9 Why using the default $\chi^{2}$ method would be the wrong way to fit histograms in this and other efficiency studies}

It ought to be common knowledge among physicists (I thought that it is, but for some reason it is less so than I hoped) that the minimum- $\chi^{2}$ histogram fitting method, which is the default in PAW, ROOT, MN_FIT and other similar programs, has severe limitations on its applicability that have to do mostly with the fact that the number of entries in a histogram's bin is a Poisson-distributed value, not Gaussian. BAD 318, "Recommended Statistical Procedures for BABAR" [87], briefly touches this problem and recommends that the alternative likelihood-based method be used when the number of events per bin is "small". It does not, however, provide a convincing example on how badly one can screw up by using the $\chi^{2}$ method. I provide such an example here.

The file tools/chi2test.hbook contains four histograms, each with 1000 bins from 0.0 to 1.0 , filled with a uniform random-number generator with $10 \mathrm{k}, 20 \mathrm{k}, 100 \mathrm{k}$, and $200 \mathrm{k}$ events, so that the average number of events per bin is, respectively, 10, 20, 100 and 200. Let us try to extract the number of events in each of the four histograms 
by fitting them with a constant and then use the fit results to compute a double ratio that should equal $R=(10 / 20) /(100 / 200)=1$. This, after all, is the kind of calculation we have to do in our $K_{S}^{0}$ efficiency study. A $\chi^{2}$ fit with a constant, such as

h/fit 1 PO

in PAW, returns (surprise!) $8.9195 \pm 0.094$ where 10.0 is "expected", $18.914 \pm 0.138$ instead of $20.0,99.051 \pm 0.315$ instead of 100.0 , and $199.04 \pm 0.446$ instead of 200.0 ; we end up with $R=0.948$, which far, far from what we want. On the other hand,

h/fit 1 PO L ,

which invokes the alternative likelihood-based algorithm, produces exactly the expected result, $R=1$.

The bottom line is: if it is the number of events in a histogram that you are trying to extract, you should go with the likelihood fitter even if the numbers of events per bin are not in the single digits and even if the results of a $\chi^{2}$ fit look great to the eye. 


\section{Bibliography}

[1] J. H. Christenson, J. W. Cronin, V. L. Fitch and R. Turlay, "Evidence for the $2 \pi$ Decay of the $K_{2}^{0}$ Meson," Phys. Rev. Lett. 13, 138 (1964); also see information about the discovery of $C P$ violation at the Nobel Foundation website, http:// www .nobel.se/physics/laureates/1980/

[2] A. Alavi-Harati et al. [KTeV Collaboration], "Observation of direct $C P$ violation in $K_{S, L} \rightarrow \pi \pi$ decays," Phys. Rev. Lett. 83, 22 (1999) [arXiv:hep-ex/9905060].

[3] V. Fanti et al. [NA48 Collaboration], "A new measurement of direct $C P$ violation in two pion decays of the neutral kaon,” Phys. Lett. B 465, 335 (1999) [arXiv:hepex/9909022].

[4] An excellent introduction to the theory of Big Bang, including a discussion of inflationary cosmology, can be found in S. W. Hawking, "A Brief History Of Time: The Illustrated, Updated and Expanded Edition,” 1996.

[5] A. D. Sakharov, "Violation of $C P$ Invariance, $C$ Asymmetry, and Baryon Asymmetry of the Universe,” Pisma Zh. Eksp. Teor. Fiz. 5, 32 (1967) [JETP Lett. 5, 24 
(1967 SOPUA,34,392-393.1991 UFNAA,161,61-64.1991)].

[6] The original article is A. H. Guth, "The Inflationary Universe: A Possible Solution To The Horizon And Flatness Problems," Phys. Rev. D 23, 347 (1981). For an accessible, in-depth first-hand account of the inflation theory, see A. H. Guth and A. P. Lightman, "The Inflationary Universe: The Quest for a New Theory of Cosmic Origins," 1997.

[7] The two most recently published results on the search for proton decay are M. Shiozawa et al. [Super-Kamiokande Collaboration], "Search for proton decay via $p \rightarrow e^{+} \pi^{0}$ in a large water Cherenkov detector," Phys. Rev. Lett. 81, 3319 (1998) [arXiv:hep-ex/9806014] and C. McGrew et al., "Search For Nucleon Decay Using The Imb-3 Detector," Phys. Rev. D 59, 052004 (1999).

[8] D. E. Groom et al. [Particle Data Group Collaboration], "Review Of Particle Physics," Eur. Phys. J. C 15, 1 (2000).

[9] K. Hagiwara et al. [Particle Data Group Collaboration], "Review Of Particle Physics," Phys. Rev. D 66, 010001 (2002).

[10] See Production and Decay of b-flavored Hadrons in [9].

[11] For a review of the Standard Model, see Standard Model and Related Topics in [9] and references therein.

[12] See CKM quark-mixing matrix in [9]. 
[13] See CP violation in B decays - Standard Model predictions in [9].

[14] M. Kobayashi and T. Maskawa, " $C P$ Violation in the Renormalizable Theory of Weak Interaction,” Prog. Theor. Phys. 49, 652 (1973).

[15] H. Y. Cheng, “The Strong CP Problem Revisited,” Phys. Rept. 158, 1 (1988).

[16] P. G. Harris et al., "New Experimental Limit On The Electric Dipole Moment Of The Neutron,” Phys. Rev. Lett. 82, 904 (1999).

[17] T. Lee, "Resolution of strong $C P$ problem," Int. J. Mod. Phys. A 16, 4321 (2001) [arXiv:hep-ph/0006349].

[18] H. Banerjee, D. Chatterjee and P. Mitra, "Is there still a strong $C P$ problem?", Phys. Lett. B 573, 109 (2003) [arXiv:hep-ph/0012284].

[19] L. Wolfenstein, "Parametrization Of The Kobayashi-Maskawa Matrix," Phys. Rev. Lett. 51, 1945 (1983).

[20] B. Aubert et al. [BABAR Collaboration], "Measurement of the $C P$-violating asymmetry amplitude $\sin 2 \beta$," Phys. Rev. Lett. 89, 201802 (2002) [arXiv:hepex/0207042]

[21] K. Abe et al. [Belle Collaboration], "Measurement of $C P$-violation parameter $\sin \left(2 \phi_{1}\right)$ with 152 million $B \bar{B}$ pairs," arXiv:hep-ex/0308036. 
[22] J. Charles, A. Höcker, H. Lacker et al. [CKMfitter Working Group], "Plots and Results, Lepton-Photon 2003,” http://www.slac.stanford.edu/ laplace/ ckmfitter/ckm_results_summer03.html.

[23] A. Höcker, H. Lacker, S. Laplace and F. Le Diberder, "A new approach to a global fit of the CKM matrix,” Eur. Phys. J. C 21, 225 (2001) [arXiv:hep-ph/0104062].

[24] http://www.slac.stanford.edu/ laplace/ckmfitter/ckm_inputs2003. html (currently under construction—refer to ckm_inputs2002.html until the above link becomes available.

[25] N. G. Deshpande and J. Trampetic, "Penguin Mediated Exclusive Hadronic Weak $B$ Decays,” Phys. Rev. D 41, 895 (1990).

[26] N. G. Deshpande and X. G. He, "Gluonic penguin $B$ decays in Standard and two Higgs doublet Models," Phys. Lett. B 336, 471 (1994) [arXiv:hep-ph/9403266].

[27] R. Fleischer, "Electroweak Penguin effects beyond leading logarithms in the $B$ meson decays $B^{-} \rightarrow K^{-} \phi$ and $B^{-} \rightarrow \pi^{-} \bar{K}^{0}$," Z. Phys. C 62, 81 (1994).

[28] Y. Grossman and M. P. Worah, " $C P$ asymmetries in $B$ decays with new physics in decay amplitudes,” Phys. Lett. B 395, 241 (1997) [arXiv:hep-ph/9612269].

[29] R. Fleischer, " $C P$ violation and the role of electroweak penguins in non-leptonic $B$ decays,” Int. J. Mod. Phys. A 12, 2459 (1997) [arXiv:hep-ph/9612446]. 
[30] I. Hinchliffe and N. Kersting, "Constraining $C P$ violating phases of the MSSM," Phys. Rev. D 63, 015003 (2001).

[31] M. Ciuchini and L. Silvestrini, "Direct $C P$ violation in $B \rightarrow \phi K_{S}^{0}$ and new physics," Phys. Rev. Lett. 89, 231802 (2002).

[32] S. Mishima and A. I. Sanda, "Calculation of magnetic penguin amplitudes in $B \rightarrow \phi K$ decays using PQCD approach,” Prog. Theor. Phys. 110, 549 (2003) [arXiv:hep-ph/0305073].

[33] S. Mishima and A. I. Sanda, "An Analysis of Supersymmetric Effects on $B \rightarrow \phi K$ Decays in PQCD Approach,” arXiv:hep-ph/0311068.

[34] A. K. Giri and R. Mohanta, "Direct $C P$ violation and new physics effects in the decay mode $B^{+} \rightarrow \phi K^{+}$, arXiv:hep-ph/0311011.

[35] J. F. Cheng and C. S. Huang, " $B \rightarrow \phi \pi$ in SM and MSSM," Phys. Lett. B 554, 155 (2003) [arXiv:hep-ph/0207177].

[36] S. Bar-Shalom, G. Eilam and Y. D. Yang, " $B \rightarrow \phi \pi$ and $B^{0} \rightarrow \phi \phi$ in the standard model and new bounds on $R$ parity violation," Phys. Rev. D 67, 014007 (2003).

[37] H. Y. Cheng and K. C. Yang, "Analysis of $B \rightarrow \phi K$ decays in QCD factorization," Phys. Rev. D 64, 074004 (2001) [arXiv:hep-ph/0012152].

[38] C. H. Chen, Y. Y. Keum and H. n. Li, "Perturbative QCD analysis of $B \rightarrow \phi K$ decays," Phys. Rev. D 64, 112002 (2001) [arXiv:hep-ph/0107165]. 
[39] B. Aubert et al. [BABAR Collaboration], "Measurement of the decays $B \rightarrow \phi K$ and $B \rightarrow \phi K^{*}$," Phys. Rev. Lett. 87, 151801 (2001) [arXiv:hep-ex/0105001].

[40] A. Bozek [the Belle Collaboration], "Charmless $B$ decays involving vector mesons in Belle," arXiv:hep-ex/0104041.

[41] R. A. Briere et al. [CLEO Collaboration], "Observation of $B \rightarrow \phi K$ and $B \rightarrow$ $\phi K^{*}$, Phys. Rev. Lett. 86, 3718 (2001) [arXiv:hep-ex/0101032].

[42] B. Aubert et al. [BABAR Collaboration], "Direct $C P$ violation searches in charmless hadronic $B$ meson decays," Phys. Rev. D 65, 051101 (2002) [arXiv:hepex/0111087].

[43] PEP-II Conceptual Design Report, SLAC-R-418 (1993).

[44] P. F. Harrison and H. R. Quinn [BABAR Collaboration], "The BABAR physics book: Physics at an asymmetric $B$ factory," SLAC-R-0504.

[45] B. Aubert et al. [BABAR Collaboration], "The BABAR detector," Nucl. Instrum. Meth. A 479, 1 (2002) [arXiv:hep-ex/0105044].

[46] C. Bozzi et al. [BABAR Collaboration], "The Design And Construction Of The BABAR Silicon Vertex Tracker," Nucl. Instrum. Meth. A 447, 15 (2000).

[47] G. Sciolla et al. [BABAR Collaboration], "The BABAR drift chamber," Nucl. Instrum. Meth. A 419, 310 (1998). 
[48] M. H. Kelsey [BABAR Collaboration], "Performance and aging of the BABAR drift chamber," SLAC-PUB-10224, Contributed to IEEE 2003 Nuclear Science Symposium (NSS) and Medical Imaging Conference (MIC) including Sessions on Nuclear Power Systems, Portland, Oregon, 19-24 Oct 2003.

[49] J. Va’vra, "Review Of Wire Chamber Aging," Nucl. Instrum. Meth. A 252, 547 (1986).

[50] L. Malter, “Thin Film Field Emission,” Phys. Rev. 50, 48 (1936).

[51] J. Schwiening et al. [BABAR-DIRC Collaboration], "The DIRC detector at the SLAC B-factory PEP-II: Operational experience and performance for physics application,” Nucl. Instrum. Meth. A 502, 67 (2003).

[52] I. Adam et al. [BABAR-DIRC Collaboration], "DIRC, the internally reflecting ring imaging Cherenkov detector for BABAR,” IEEE Trans. Nucl. Sci. 45, 657 (1998) [arXiv:hep-ex/9712001].

[53] http://www.slac.stanford.edu/BFROOT/www/Detector/DIRC/ Barbox_Sensors/.

[54] T. Hryn'ova, P. Kim, M. L. Kocian et al. [BABAR-EMC Collaboration], "A Systematic study of radiation damage to large crystals of $\mathrm{CsI}(\mathrm{Tl})$ in the BABAR detector," SLAC-PUB-10169 Prepared for 10th International Conference on Calorimetry in High Energy Physics (CALOR 2002), Pasadena, California, 25-30 Mar 2002. 
[55] F. Anulli et al. [BABAR Collaboration], "The BABAR Instrumented Flux Return Performance: Lessons Learned,” Nucl. Instrum. Meth. A 494, 455 (2002).

[56] M. Andreotti, D. Bettoni, R. Calabrese et al. [The BABAR LST Team], "A Barrel IFR Instrumented with Limited Streamer Tubes," Proposal from the BABAR Collaboration to the SLAC Experimental Program Advisory Committee, May 30, 2003.

[57] V. Halyo [BABAR Collaboration], "BABAR level 1 drift chamber trigger upgrade," eConf C020620, THAP09 (2002) [arXiv:hep-ex/0209063].

[58] R. Jacobsen et al. [BABAR Collaboration], "The BABAR Event Building and Level-3 Trigger Farm Upgrade," eConf C0303241, MOGT003 (2003) [arXiv:physics/0305136].

[59] T. J. Adye et al. [BABAR Computing Group Collaboration], "KANGA(ROO): Handling the micro-DST of the BABAR experiment with ROOT," Comput. Phys. Commun. 150, 197 (2003) [arXiv:physics/0206067].

[60] R. A. Fisher, "The Use Of Multiple Measurements In Taxonomic Problems," Annals Eugen. 7, 179 (1936).

[61] A. V. Telnov [BABAR Collaboration], " $B \rightarrow \phi K^{(*)}$ : Branching Ratios and Direct CP Violation," BABAR-TALK-02/060, available at https://oraweb.slac. stanford.edu:8080/pls/slacquery/babar_documents.startup, Presented at the Annual Meeting of the Division of Particles and Fields, American Physical Society (DPF 2002), Willamsburg, VA, May 2002. 
[62] J. D. Jackson, "Remarks On The Phenomenological Analysis Of Resonances," Nuovo Cim. 34, 1644 (1964).

[63] F. Von Hippel and C. Quigg, "Centrifugal-Barrier Effects In Resonance Partial Decay Widths, Shapes, And Production Amplitudes," Phys. Rev. D 5, 624 (1972).

[64] H. Albrecht et al. [ARGUS Collaboration], Phys. Lett. B 241, 278 (1990).

[65] A. Ballaminut et al., "WIRED - World Wide Web Interactive Remote Event Display," SLAC-REPRINT-2000-033 Prepared for International Conference on Computing in High-Energy Physics and Nuclear Physics (CHEP 2000), Padova, Italy, 7-11 Feb 2000.

[66] Design of the Tracking Reconstruction Software hypernews forum, thread \#810.

[67] A. Gritsan, "10-series Momentum Charge Bias," http://www.slac. stanford.edu/BFROOT/www/Computing/Offline/Reconstruction/Tracking/ qbias_tdr2001.html.

[68] M. B. Voloshin, "Variation of the relative yield of charged and neutral $B$ mesons across the $\Upsilon(4 S)$ resonance," Mod. Phys. Lett. A 18, 1783 (2003) [arXiv:hep$\mathrm{ph} / 0301076]$.

[69] A. Gritsan, "Boost Effect on $\Delta E$ separation," http://www.slac.stanford.edu/ oritsan/deltaE/index.html.

[70] C. Dallapiccola, private communication (November 2002). 
[71] K. Abe et al. [Belle Collaboration], "Study of charmless B decays to three-kaon final states," arXiv:hep-ex/0208030.

[72] J. P. Alexander, p. Chang, J. G. Smith et al. [Heavy Flavor Averaging Group], http://www.slac.stanford.edu/xorg/hfag/rare/index.html.

[73] A. Bertin et al. [OBELIX Collaboration], "Study Of The $f_{0}(1500) / f_{2}(1565)$ Production In The Exclusive Annihilation $\bar{n} p \rightarrow \pi^{+} \pi^{+} \pi^{-}$In Flight," Phys. Rev. D 57, 55 (1998).

[74] B. Aubert et al. [BABAR Collaboration], "Measurement of branching fractions for exclusive $B$ decays to charmonium final states," Phys. Rev. D 65, 032001 (2002) [arXiv:hep-ex/0107025].

[75] S. B. Athar et al. [CLEO Collaboration], "Measurement of the ratio of branching fractions of the $\Upsilon(4 S)$ to charged and neutral $B$ mesons," Phys. Rev. D 66, 052003 (2002) [arXiv:hep-ex/0202033].

[76] B. Aubert et al. [BABAR Collaboration], "Time-dependent $C P$-Violating Asymmetries in $B^{0} \rightarrow \phi K_{S}^{0}$, BABAR-PLOT-0056, available at https://oraweb.slac. stanford.edu:8080/pls/slacquery/babar_documents.startup, which supersedes the original preliminary result, B. Aubert et al. [BABAR Collaboration], "Measurement of $\sin (2 \beta)$ in $B^{0} \rightarrow \phi K_{S}^{0}$ ", arXiv:hep-ex/0207070.

[77] K. Abe et al. [Belle Collaboration], "Measurement of time-dependent CPviolating asymmetries in $B^{0} \rightarrow \phi K_{S}^{0}, K^{+} K^{-} K_{S}^{0}$, and $\eta^{\prime} K_{S}^{0}$ decays," arXiv:hep- 
ex/0308035, which supersedes the original preliminary result, K. Abe et al. [Belle Collaboration], "An improved measurement of mixing-induced $C P$ violation in the neutral $B$ meson system,” arXiv:hep-ex/0207098.

[78] W. T. Ford, BAD 53, "Choice of Kinematic Variables in $B$ Meson Reconstruction-Take 3" (June 2000).

[79] F. Martinez-Vidal, M. Carpinelli et al., BAD 102, "The BABAR Vertexing” (December 2001).

[80] G. Mancinelli, S. M. Spanier, BAD 116, "Kaon Selection at the BABAR Experiment" (July 2001).

[81] Based on the method described in C. Hearty, BAD 134, "Measurement of the Number of $\Upsilon(4 S)$ Mesons Produced in Run 1 (B Counting)" (January 2001).

[82] A. V. Gritsan, BAD 156, "Four-Body Charmless $B$ Decays. Observation of $B \rightarrow$ $\phi K^{* ”}$ (May 2001).

[83] G. Cavoto, C. Dallapiccola et al., BAD 160, "Analysis of Neutral B Decays to $\pi^{+} \pi^{-}, \pi^{+} K^{-}$and $K^{+} K^{-}$(March 2001).

[84] D. C. Williams, BAD 228, "Measurements of the $D^{0}$ Branching Ratios, Lifetimes, and Mixing Using Two-Prong Decays" (March 2002).

[85] S. Spanier, BAD 303, "Parametrization of the Dynamics of Partial Waves" (November 2001). 
[86] S. Spanier, D. Dujmic, M. Krishnamurthy, BAD 313, "Measurement of timedependent $C P$ asymmetries in $B \rightarrow \phi K "$ (February 2003).

[87] F. C. Porter et al., BAD 318, "Recommended Statistical Procedures for BABAR" (May 2002).

[88] E. Varnes et al., BAD 324, "Report of the Tracking Efficiency Task Force for 2001" (October 2002).

[89] F. Bianchi et al., BAD 357, "Measurement of branching fractions and $C P$-violating asymmetries in $B \rightarrow h^{+} h^{-}$decays" (March 2002).

[90] M. Bona et al., BAD 372, "Measurement of Branching Fractions for Charmless $B^{+} \rightarrow h^{\prime+} h^{0}$ Decays" (May 2002).

[91] N. Berger et al., BAD 390, "Exclusive Charmless Two-Body $B$ Decays to $\eta$ and $\eta^{\prime}$ in Run 1 and Run 2 Data” (May 2002).

[92] T. Brandt, BAD 396, "Likelihood Based Electron Identification" (March 2002).

[93] C. Roat, BAD 402, "Identification of Hadrons for $\Lambda_{c}$ Reconstruction in BABAR" (June 2002).

[94] A. V. Telnov, A. V. Gritsan, BAD 406, "Branching Ratios and Direct CP Violation in $B \rightarrow \phi K "$ (February 2003).

[95] D.C. Williams, W. Lockman, BAD 428, "Systematic Studies of the $D^{0}$ mass from the decay $K \pi$ " (June 2002). 
[96] M. Bona, G. Cavoto, C. Dallapiccola et al., BAD 446, "Measurement of Branching Fractions and $C P$-Violating Asymmetries in $B^{0} \rightarrow h^{+} h^{\prime-}$ Decays" (July 2002).

[97] A. V. Gritsan, BAD 498, "Rates, Polarizations, and Asymmetries in $B \rightarrow \phi K^{*}$ Decays" (July 2003).

[98] J.J. Back, BAD 505, "Studies of the $B^{+} \rightarrow h^{+} h^{-} h^{+}(h=\pi / K)$ Decays on the Full Summer 2002 Dataset” (January 2003).

[99] F. Blanc, P. Bloom, I. M. Derrington, W. T. Ford, W. C. van Hoek, C. L. Lee, J. G. Smith, K. A. Ulmer, BAD 521, "Exclusive Charmless Two-Body B Decays to $\eta$ and $\eta^{\prime}$ Mesons" (June 2003).

[100] F. Blanc, P. Bloom, W. T. Ford, W. C. van Hoek, J. G. Smith, L. Zhang, BAD 528, "Exclusive Charmless Two-Body B Decays to $\omega$ meson in Run 1 and Run 2 Data" (February 2003).

[101] J. W. Berryhill, F. C. Porter, J. D. Richman, A. Ryd, A. Samuel, BAD 559, “Observation of the Rare Decays $B \rightarrow K \ell^{+} \ell^{-}$and $B \rightarrow K^{*} \ell^{+} \ell^{-}$" (July 2003).

[102] F. Blanc, private communication (September 2002).

[103] http://www.slac.stanford.edu/BFR00T/www/Physics/Tools/Pid/ PidKilling/PidKilling.html.

[104] http://www.slac.stanford.edu/〜aquinas/internal/tmp-2002-09-04/ Kshort.html 
[105] http://www-zeus.physik.uni-bonn.de/ brock/mn_fit.html 